\title{
The Interaction of Intense Subpicosecond Laser Pulses with Underdense Plasmas
}

C. A. Coverdale

(Ph.D. Thesis)

Manuscript date: May 11, 1995

\section{LAWRENCE LIVERMORE NATIONAL LABORATORY University of California - Livermore, California • 94551}

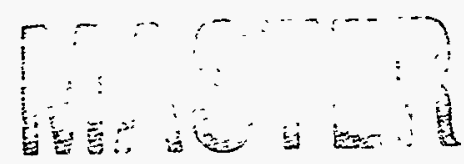




\section{DISCLAIMER}

Portions of this document may be illegible in electronic image products. Images are produced from the best available original document. 
The Interaction of Intense Subpicosecond Laser

Pulses with Underdense Plasmas

By

Christine Ann Coverdale

BS, University of Puget Sound, 1988

MS, University of California, Davis 1989

\section{DISSERTATION}

Submitted in partial satisfaction of the requirements for the degree of DOCTOR OF PHILOSOPHY

in

Applied Science

in the

GRADUATE DIVISION

of the

\section{UNIVERSITY OF CALIFORNIA}

DAVIS

Approved:

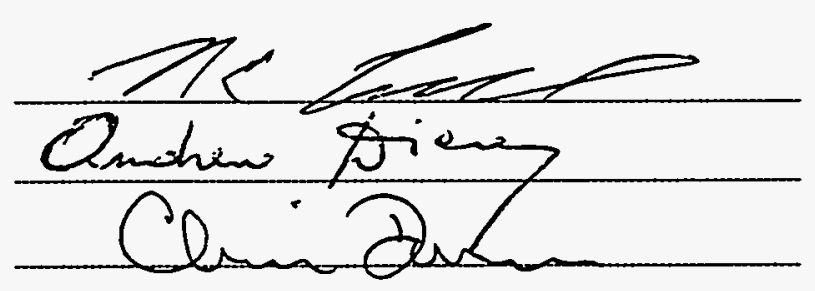

Committee in Charge

1995 


\section{Acknowledgements}

There are many individuals I would like acknowledge for their contributions and guidance over the last few years. First and foremost, I would like to thank Dr. Chris Darrow, my thesis advisor, who spent countless hours teaching me about plasma physics both in and out of the lab. I learned more from him in the last few years than I can even begin to express. I would also like to thank Mike Perry and the members of his group for the generous use of their laser, lab space, and equipment. The experiments presented in this dissertation would not have been possible without them. Hoang Nguyen and John Crane deserve special recognition not only for their assistance with the laser and the experiments, but also for their enthusiasm, encouragement, and moral support.

While working to understand the experimental data, I had the pleasure of many conversations with the theorists and computational physicists of X-division at the Lawrence Livermore National Lab. Three individuals deserve particular thanks: Denise Hinkel, Bill Kruer, and Scott Wilks. They were always willing to spend time with me explaining theories and simulations, helping immensely in my understanding of stimulated Raman scattering and plasma physics. Their expertise was invaluble in the modeling and understanding of the experiments.

Some of the work presented in this thesis resulted from a productive collaboration with members of the UCLA Physics and Electrical Engineering Departments and I would be remiss if I did not acknowledge their contributions. On the experimental side, Chan Joshi, Chris Clayton, and Ken Marsh provided both equipment and expertise. Their help was instrumental in obtaining the electron measurements discussed in this work. Warren Mori, Chris Decker, and Kuo-Cheng Tzeng performed 
computer simulations which closely modeled the experiments, helping tremendously in the selection of experimental parameters in the forward stimulated Raman experiments. They also helped to provide explanations for anomalous features observed in the experiments.

I would also like to thank the Fannie and John Hertz Foundation for helping to ease the financial burden of graduate school.

On a more personal note, there are several people who deserve acknowledgement for helping me maintain my sanity during my years as a graduate student. My family stood by me and provided moral support through both the good and bad times. Kudos go to my local family: Doug Miller, Debbie Miller, Dale Slone, and Kim Budil. Doug, Debbie, and Kim have been there since the very beginning and I'm glad to say they are around to see the end. The "Doctors Miller" and the "Blones" have been in large part responsible for the life I have outside the lab.

My best friend, Kevin Baker, deserves more thanks than I can possibly write here for both personal and professional reasons. As a fellow student of laser-plasma physics, Kevin has been a fountain of knowledge at times and I have picked his brain repeatedly. He has listened endlessly and tried to do what he could to help when the chips were down. His encouragement, friendship, and advice helped me persevere to make this thesis a reality.

There are many others who have helped make graduate school an interesting and educational experience. I learned how to play poker without going broke (thanks Jeff, Gwen, Jay, Brian, and Bob), spent many an evening playing softball (courtesy of the Lost Dwarves), and have become an expert in frustration while attempting volleyball (as a member of the Outsiders and on that asphalt court). Overall, I can certainly say the last few years would not have been nearly as interesting without the friends I made through the Department of Applied Science. Thanks, guys. 


\section{Contents}

Acknowledgements $\quad$ iii

List of Tables vii

List of Figures viii

1 Introduction 3

2 Theory of Stimulated Raman Scattering 12

2.1 Basic theory of Stimulated Raman Scattering ... . . . . . . 13

2.2 The Coupled Equations . . . . . . . . . . . . . . . . . . 19

2.3 The Dispersion Relation . . . . . . . . . . . . . . . 34

2.3 .1 Nonrelativisitic . . . . . . . . . . . . . 35

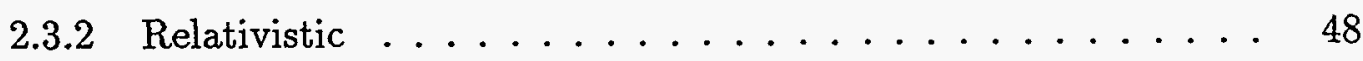

2.4 Summary $\ldots \ldots \ldots \ldots \ldots \ldots \ldots \ldots \ldots \ldots \ldots$

3 Experimental Apparatus and Parameters $\quad 55$

3.1 System Overview . . . . . . . . . . . . . 55

3.2 The Laser System . . . . . . . . . . . . . . . . . 58

3.3 Light Collection Diagnostics . . . . . . . . . . . . . . . . 64

3.4 Electron Energy Diagnostics . . . . . . . . . . . . . . 66

3.5 Plasma Creation and Characterization . . . . . . . . . 69

3.5.1 Creation of the Plasma . . . . . . . . . . . . . 69 


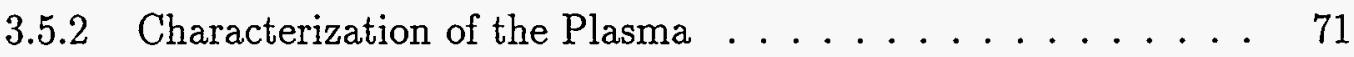

4 Forward Stimulated Raman Scatter $\quad 75$

4.1 Parameter Regime . . . . . . . . . . . . . 75

4.2 Forward Stimulated Raman Scatter Spectra _ . . . . . . . . . 77

4.3 Energetic Electron Measurements .............. 83

4.3.1 $\mathrm{K}_{\alpha}$ Flourescence Measurement . . . . . . . . . . . 84

4.3.2 Magnetic Electron Spectrometer Measurement . . . . . . . . 89

4.4 Energy transmitted through the underdense plasma . . . . . . . . . 92

4.5 Implications of Forward SRS Observations . . . . . . . . . . 95

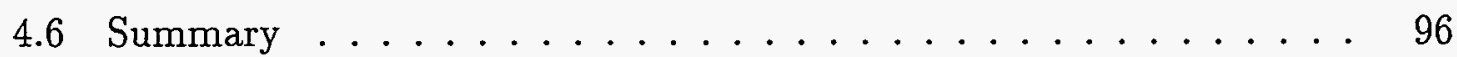

5 Stimulated Raman Backscatter $\quad 97$

5.1 Parameter regime . . . . . . . . . . . . . . . 97

5.2 General Behavior of Backscattered SRS . . . . . . . . . . . 99

5.3 Spectral Features . . . . . . . . . . . . . . . 101

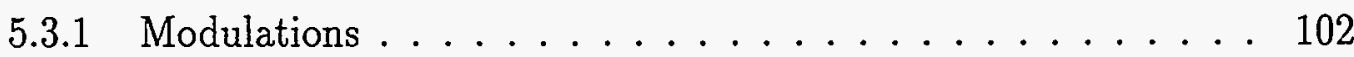

5.3.2 Broad Feature Underlying the Modulations . . . . . . . . . 105

5.3 .3 Reflectivity ................... 109

5.4 Candidate Mechanisms for Modulations . . . . . . . . . . . . . 113

5.5 Anomalous Features at Higher Densities . . . . . . . . . 118

5.6 Summary ........................... 125

$\begin{array}{lll}6 & \text { Summary } & 127\end{array}$

A Plasma Images $\quad 131$

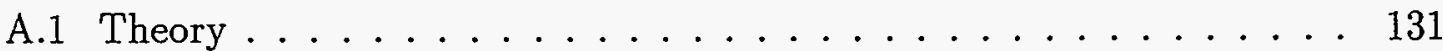

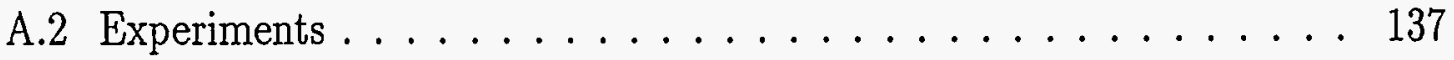

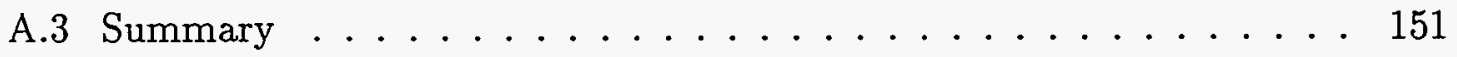

$\begin{array}{ll}\text { B } & 152\end{array}$ 


\section{List of Tables}

1.1 Parameters of some long and short pulse laser systems $\ldots \ldots \ldots 7$

5.1 Values of the density fluctuation, $\delta \mathrm{n} / \mathrm{n}$, for a variety of plasma densities and incident laser intensities. . . . . . . . . . . . 112 


\section{List of Figures}

1.1 Illustration of relative sizes of laser pulse length, interaction regime, and plasma length for (a) the short pulse, high intensity laser regime (b) the long pulse, moderate intensity laser regime. . . . . . . . .

2.1 (a) Illustration of decay process of SRS and (b) schematic of the SRS "feedback" loop. . . . . . . . . . . . . . . . . . . . . 13

2.2 Wavenumber matching ( $\mathrm{k}$-matching) for (a) stimulated Raman backscatter and (b) stimulated Raman forward scatter . . . . . . . . . . 14

2.3 Results of the backscatter envelope approximation. (a) Initial and final positions of the propagating laser pulse, (b) evolution of the growing electron plasma wave, (c) evolution of the scattered light wave, and (d) temporal evolution of the laser, the electron plasma wave, and the scattered light wave. These curves were obtained for $\mathrm{I}=1 \times 10^{17} \mathrm{~W} / \mathrm{cm}^{2}$ and $\mathrm{n}_{0}=7 \times 10^{17} \mathrm{~cm}^{-3} \ldots \ldots \ldots \ldots \ldots \ldots$

2.4 Growth of the electron plasma wave as a function of (a) peak laser intensity $\left(\mathrm{n}_{0}=1 \times 10^{17} \mathrm{~cm}^{-3}\right)$ and (b) plasma density ( $\mathrm{I}=7 \times$ $\left.10^{16} \mathrm{~W} / \mathrm{cm}^{2}\right)$. The propagation time was 4000 fsec. . . . . . . .

2.5 Solution to coupled equations for a random initial density perturbation.

(a) The random perturbation, (b) evolution of the electron plasma wave, and (c) evolution of the scattered light wave. In this calculation, $\mathrm{n}_{0}=7 \times 10^{17} \mathrm{~cm}^{-3}, \mathrm{I}=1 \times 10^{17} \mathrm{~W} / \mathrm{cm}^{2}$. The laser propagated for 4000 fsec. . . . . . . . . . . . . . . . . . . . 
2.6 (a) Roots of the fourth order dispersion relation for an undriven system $\left(v_{\text {osc }} / c=0, n_{0}=1 \times 10^{18} \mathrm{~cm}^{-3}\right)$; (b) roots of the dispersion relation for a driven system ( $\mathrm{v}_{\mathrm{osc}} / \mathrm{c}=0.5, \mathrm{n}_{0}=1 \times 10^{18} \mathrm{~cm}^{-3}$ ) and (c) the corresponding spectrum for the driven system. . . . . . . .

2.7 (a) Numerical spectra for $\mathrm{v}_{\mathrm{osc}} / \mathrm{c}=0.8$ and 0.2 , (b) numerical spectra for $\mathrm{n}_{0}=1 \times 10^{17}$ and $1 \times 10^{18} \mathrm{~cm}^{-3}$, and the growth rate of backscattered stimulated Raman scattering as a function of intensity (c) and density (d). . . . . . . . . . . . . . . . . .

2.8 (a) Noise source used to obtain spectrum (c); (b) random noise source used to generate spectrum (d). For both spectra, $\mathrm{v}_{\text {osc }} / \mathrm{c}=0.8$ and $\mathrm{n}_{0}=7 \times 10^{17} \mathrm{~cm}^{-3} \ldots \ldots \ldots \ldots \ldots \ldots \ldots \ldots$

2.9 "Detected" spectrum obtained using $\omega_{0}-\omega_{\text {det }} / \omega_{\text {pe }}$ and the uncorrected spectrum $\omega_{\mathrm{r}} / \omega_{\mathrm{pe}}$. In both cases, $\mathrm{v}_{\mathrm{osc}} / \mathrm{c}=0.8$ and $\mathrm{n}_{0}=7 \times 10^{17} \mathrm{~cm}^{-3}$.

2.10 Comparison of solutions of the dispersion relation when the anti-Stokes term is neglected (dashed line) and included (solid line). . . . . . .

2.11 Roots of the (a) undriven $\left(\mathrm{v}_{\mathrm{osc}} / \mathrm{c}=0\right)$ and $(\mathrm{b})$ driven $\left(\mathrm{v}_{\mathrm{osc}} / \mathrm{c}=0.7\right)$ sixth order dispersion relation. For both cases, $\mathrm{n}_{0}=1 \times 10^{19} \mathrm{~cm}^{-3}$. .

2.12 Spectrum obtained from the sixth order dispersion relation for forward stimulated Raman scattering. In this case, $v_{\text {osc }} / c=1$ and $n_{0}=2 \times$ $10^{19} \mathrm{~cm}^{-3} \ldots \ldots \ldots \ldots \ldots \ldots \ldots \ldots \ldots \ldots \ldots \ldots$

2.13 (a) Spectrum of forward SRS for $\mathrm{v}_{\text {osc }} / \mathrm{c}=0.5$ (solid) and 0.8 (dashed);

(b) forward SRS spectrum for $\mathrm{n}_{0}=7 \times 10^{18} \mathrm{~cm}^{-3}$ and $2 \times 10^{19} \mathrm{~cm}^{-3}$;

(c) growth as a function of incident intensity (solid triangles); and (d) growth as a function of plasma density (solid triangles). In plots (c) and $(\mathrm{d})$, the solid line was obtained using equation $(2.77) . \ldots \ldots$

2.14 "Detected" spectrum obtained with the relativistic dispersion relation and correction (solid) and the nonrelativistic versions (dashed). . . . 
2.15 Growth of the plasma wave as a function of incident intensity (a) and plasma density (b). The results from the nonrelativistic calculation are shown as open triangles and the solid line was calculated using the relativistic growth rate (equation (2.88)). . . . . . . . . 50

2.16 Forward SRS spectrum obtained by solving the relativistic dispersion relation (solid) and the nonrelativistic dispersion relation (dashed) for $\mathrm{n}_{0}=1 \times 10^{19} \mathrm{~cm}^{-3}$ and $\mathrm{v}_{\mathrm{osc}} / \mathrm{c}=0.8 \ldots \ldots \ldots \ldots \ldots$

2.17 Incident intensity (a) and plasma density (b) dependence of the growth of forward SRS (solid circles). The results obtained with the nonrelativistic dispersion relation are shown as open triangles. The solid line

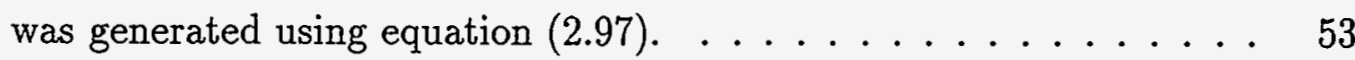

3.1 Experimental Configurations . . . . . . . . . . . . . 56

3.2 Schematic of the $10 \mathrm{TW}$ laser system . . . . . . . . . . . 59

3.3 Amplified spectrum of the $10 \mathrm{TW}$ laser . . . . . . . . . . . 61

3.4 Autocorrelation trace of unamplified laser pulse . . . . . . . . 62

3.5 Contour plots of the laser spot before and after best focus . . . . . 63

3.6 Energy of electrons reaching surface barrier detector and field of the deflecting as a function of current applied to the magnet . . . . . 68

3.7 Low intensity SRS spectrum obtained with a helium static fill pressure

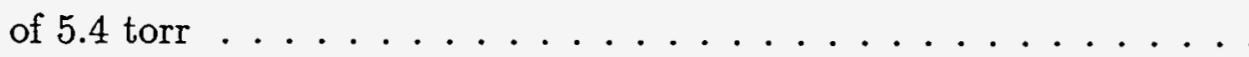

3.8 (a) Wavelength shift of SRS feature as a function of helium static fill pressure. (b) Density inferred from SRS shift versus density of known

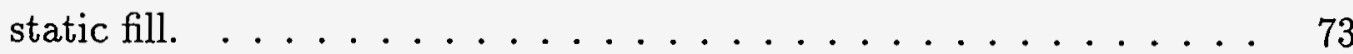

3.9 Variation of electron density with backing pressure of the gas jet . . 74

4.1 Regime in which forward SRS can be observed . . . . . . . . 76

4.2 Spectrum of near-forward scattered light showing scattered laser light, and the first and second anti-Stokes features of forward stimulated Raman scattering. The dashed line shows the result predicted from the dispersion relation (retaining only first Stokes and anti-Stokes). . 
4.3 (a) The density inferred from the first anti-Stokes mode (open circles) and the backscattered SRS signal (solid diamonds) and (b) the frequency shift of the first anti-Stokes mode as a function of plasma density. . . . . . . . . . . . . . . . . 81

4.4 Amplitude of the first anti-Stokes feature as a function of (a) incident laser intensity (fixed backing pressure, 800 psi helium) and (b) plasma density (fixed incident intensity, $\mathrm{v}_{\text {osc }} / \mathrm{c} \sim 0.6$ ) $\ldots \ldots \ldots \ldots$

4.5 Forward SRS spectra obtained with increasing density. The laser intensity was fixed at $\mathrm{v}_{\mathrm{osc}} / \mathrm{c}=0.8 \ldots \ldots \ldots \ldots \ldots$

4.6 Schematic of the $\mathrm{K}_{\alpha}$ emission process $\ldots \ldots \ldots \ldots \ldots$

4.7 Example picture of the $\mathrm{K}_{\alpha} \mathrm{x}$-ray 'hits' recorded by the CCD. . . . 85

4.8 X-ray energy spectra obtained using (a) $25 \mu \mathrm{m} \mathrm{Sn}$ and (b) $25 \mu \mathrm{m} \mathrm{Sn}$ $+50 \mu \mathrm{m}$ Mo. The $K_{\alpha}$ peak for $\mathrm{Sn}$ is at $25.3 \mathrm{keV} \ldots \ldots \ldots$

4.9 (a) Experimental setup used to study radial extent of the energetic electrons, and (b) $K_{\alpha}$ emission as a function of radial distance from the laser axis. . . . . . . . . . . . . . .

4.10 Signal levels on the electron and control detectors. The open triangles show the "null" experiment values. . . . . . . . . . . . . . . 9 90

4.11 Correlation of electron signal with first anti-Stokes feature (open circles). The solid triangles indicate the "null" experiment. . . . . . . 91

$4.122 .0 \mathrm{MeV}$ electron signal as a function of gas jet backing pressure. . . . 92

4.13 (a) Transmission of incident laser energy in the focal cone of the laser as a function of incident energy. (b) Correlation between the observation of anti-Stokes features and the decrease in transmission. . . . . . . 94

5.1 Density and intensity regime for which backscatterd stimulated Raman scatter can grow (shaded area). The dashed line defines the region in which forward SRS will be observable. . . . . . . . . . . 
5.2 Backscattered stimulated Raman backscatter spectra obtained with plasma density of $\mathrm{n}_{0}=6 \times 10^{17} \mathrm{~cm}^{-3}$ and an intensity range of $5 \times$ $10^{16} \mathrm{~W} / \mathrm{cm}^{2}$ to $3 \times 10^{17} \mathrm{~W} / \mathrm{cm}^{2} \ldots \ldots \ldots 100$

5.3 Backscattered SRS spectra obtained with plasma densities ranging from $1.4 \times 10^{17} \mathrm{~cm}^{-3}$ to $1.3 \times 10^{18} \mathrm{~cm}^{-3}$ and a fixed laser intensity of $1.7 \times 10^{17} \mathrm{~W} / \mathrm{cm}^{2}$. . . . . . . . . . . . . . . 101

5.4 Three consecutive backscattered SRS spectra obtained for identical laser and plasma conditions. The laser intensity was $2.5 \times 10^{17} \mathrm{~W} / \mathrm{cm}^{2}$ and the plasma density was $6.7 \times 10^{17} \mathrm{~cm}^{-3} \ldots \ldots \ldots 3$

5.5 (a) Spectrum (in $\omega$-space) obtained for $\mathrm{n}_{0}=6 \times 10^{17} \mathrm{~cm}^{-3}$ and $\mathrm{v}_{\text {osc }} / \mathrm{c}=$ 0.46 , and (b) the corresponding symmetry of the modulation peaks. . 104

5.6 Calculated (dashed line) and experimentally observed (solid line) spectra for $\mathrm{v}_{\mathrm{osc}} / \mathrm{c}=0.31$ and $\mathrm{n}_{0}=6 \times 10^{17} \mathrm{~cm}^{-3}$. The "detected" spectrum is shown in (a) and the "shifted" spectrum is shown in (b). . . . . .

5.7 The "width" and "shift" of the SRS feature can be obtained from the backscattered spectrum. . . . . . . . . . . . 106

5.8 Full width half-maximum of the broad feature underlying the modulated SRS. The solid circles show experimentally measured widths for $\mathrm{n}_{0}=7 \times 10^{17} \mathrm{~cm}^{-3}$. The open squares were obtained from spectra calculated with the relativistic dispersion relation (equation (2.88)). . 107

5.9 Distance the calculated spectra must be blue-shifted in order to overlap the experimentally observed spectra. The solid circles (open squares) show the shift for $\mathrm{n}_{0}=3 \times 10^{17} \mathrm{~cm}^{-3}\left(7 \times 10^{17} \mathrm{~cm}^{-3}\right) \ldots \ldots 108$

5.10 Reflectivity of the backscattered SRS feature as a function of incident laser intensity. The open triangles (solid circles) show the reflectivity values for $\mathrm{n}_{0}=3 \times 10^{17} \mathrm{~cm}^{-3}\left(7 \times 10^{17} \mathrm{~cm}^{-3}\right)$ with $\mathrm{L}_{\mathrm{z}}=1 \mathrm{~mm}$. The density fluctuation, $\delta \mathrm{n} / \mathrm{n}$ is given on the right hand $\mathrm{y}$-axis. . . . . . 110 
5.11 Growth rate of the backscattered SRS as a function of intensity. The solid circles represent values of $\gamma_{0}$ extracted from the reflectivity. The open triangles (circles) were calculated using the relativistic (nonrelativistic) growth rate for backscatter. . . . . . . . . . . . . . 113

5.12 Gaussian wavepackets and their corresponding spectra for constant temporal separation of the wavepackets ((a) and (b)) and random temporal wavepacket separations ((c) and (d)). . . . . . . . . . 118

5.13 Evolution of the backscattered spectrum for a fixed plasma density of $\mathrm{n}_{0}=8 \times 10^{18} \mathrm{~cm}^{-3}$. The laser intensity ranged from $1 \times 10^{17} \mathrm{~W} / \mathrm{cm}^{2}$ to $6 \times 10^{17} \mathrm{~W} / \mathrm{cm}^{2} \ldots \ldots \ldots \ldots \ldots \ldots \ldots$

5.14 Reflectivity of the red-shifted (open circles) and blue-shifted (solid circles) features in the backscattered spectrum. The plasma density was fixed at $8 \times 10^{18} \mathrm{~cm}^{-3} . \ldots \ldots \ldots \ldots \ldots \ldots \ldots$

5.15 Density and intensity regime for which SBS and forward SRS both grow sufficiently to couple. The experimentally accessible region is

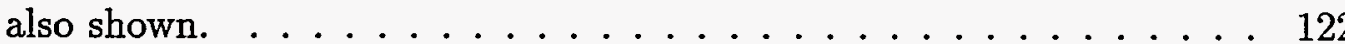

5.16 Density values inferred from the red-shifted SRS (solid circles) and the anomalous blue feature (open triangles). . . . . . . . . . . . . 124

A.1 Isointensity contours for a Gaussian distribution . . . . . . . . . 132

A.2 Spatial distribution for $\mathrm{He}^{+}$and $\mathrm{He}^{2+}$ for a peak laser intensity of $2 \times 10^{17} \mathrm{~W} / \mathrm{cm}^{2} . \ldots \ldots \ldots \ldots \ldots \ldots \ldots \ldots$

A.3 Fraction of incident energy of a focused Gaussian laser beam lost to ionization as a function of density for an infinite volume of gas. . . . 136

A.4 Length of $\mathrm{He}^{+}$and $\mathrm{He}^{2+}$ plasmas as a function of incident laser intensity.137

A.5 Experimental configuration used to collect plasma images . . . . . 138

A.6 Plasma images obtained using a $1 \omega$ filter for (a) 2.0 torr helium and (c) 510 torr helium. Contour plots of these images are shown in (b)

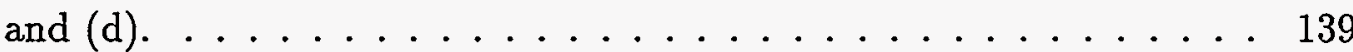


A.7 Contour plots of experimental images obtained at 510 torr helium (b) and 2 torr helium (d) and the corresponding calculated recombination brightness contour plots (a) and (c). . . . . . . . . . . . . 142

A.8 Horizontal (a) and vertical (b) lineouts of the $1 \omega$ plasma image shown in figure A.6. . . . . . . . . . . . . . . . . . . 143

A.9 Horizontal (a) and vertical (b) lineouts of a recombination light calculated image $\left(\mathrm{I}_{0}=1 \times 10^{17} \mathrm{~W} / \mathrm{cm}^{2}, \mathrm{w}_{0}=35 \mu \mathrm{m}\right) \ldots \ldots \ldots \ldots 144$

A.10 Plasma images obtained with recombination light of helium plasmas (400 torr) . . . . . . . . . . . . . . . . . . 146

A.11 Horizontal (a) and vertical (b) lineouts of the image shown in figure A.10(c). . . . . . . . . . . . . . . . . . . . . . 147

A.12 Recombination light plasma images of Argon plasmas (200 torr). . . . 149

A.13 Horizontal (a) and vertical (b) lineouts of the plasma image shown in figure A.12(a) . . . . . . . . . . . . . . . . . . . 150

B.1 (a) The frequency spectrum of the forward scattered light $\left(5^{\circ}-7^{\circ}\right.$ from the laser axis) and (b) the correlation of the $2 \mathrm{MeV}$ electron signal with the amplitude of the first anti-Stokes (open circles). The solid triangles represent signal on the electron detector when the magnet polarity was reversed (null tests). (c) The variation of the transmitted laser energy (solid triangles) and the amplitude of the first anti-Stokes (open circles) with $a_{0}$. (d) Transmitted power at all frequencies in the forward $2 \pi$ sterradians (lines) and at all frequencies in the cone angle of the beam (open symbols) versus propagation distance from PIC code simulations. The solid square represents the experimental transmission. The solid triangle is simulation result D. . . . . . . . 161

B.2 From simulation (B), the electron distribution function at (a) $0.48 \mathrm{~mm}$ and (b) $0.64 \mathrm{~mm}$ and the corresponding electromagnetic $\mathrm{k}$ spectrum at (c) $0.48 \mathrm{~mm}$ and (d) $0.64 \mathrm{~mm}\left(\mathrm{k}_{\mathrm{x}}\right.$ and $\mathrm{k}_{\mathrm{y}}$ are in units of $\omega_{\mathrm{p}} / \mathrm{c} \ldots 162$ 
Christine Ann Coverdale

May 1995

Applied Science

\begin{abstract}
The Interaction of Intense Subpicosecond Laser
Pulses with Underdense Plasmas
\end{abstract}

\title{
$\underline{\text { Abstract }}$
}

Laser-plasma interactions have been of interest for many years not only from a basic physics standpoint, but also for their relevance to numerous applications. Advances in laser technology in recent years have resulted in compact laser systems capable of generating short ( $\tau \leq 1 \mathrm{psec}$ ), intense ( $\left.\mathrm{I} \geq 5 \times 10^{16} \mathrm{~W} / \mathrm{cm}^{2}\right)$ laser pulses. These lasers have provided a new regime in which to study laser-plasma interactions, a regime characterized by $\mathrm{L}_{\text {plasma }} \geq 2 \mathrm{~L}_{\text {Rayleigh }}>c \tau$, where $\mathrm{L}_{\text {plasma }}$ is the plasma size, $\mathrm{L}_{\text {Rayleigh }}$ is the Rayleigh length, and $\mathrm{c} \tau$ is the laser pulse length.

The goal of this dissertation is to experimentally characterize the interaction of a short pulse, high intensity laser with an underdense plasma $\left(\mathrm{n}_{0} \leq 0.05 \mathrm{n}_{\mathrm{cr}}\right)$. Specifically, the parametric instability known as stimulated Raman scatter (SRS) is investigated to determine its behavior when driven by a short, intense laser pulse. Both the forward Raman scatter instability and backscattered Raman instability are studied.

The coupled partial differential equations which describe the growth of SRS are reviewed and solved for typical experimental laser and plasma parameters. This solution shows the growth of the waves (electron plasma and scattered light) generated via stimulated Raman scatter. The dispersion relation is also derived and solved for experimentally accessible parameters. The solution of the dispersion relation is used to predict where (in k-space) and at what frequency (in $\omega$-space) the instability will grow. Both the nonrelativistic and relativistic regimes of the instability are considered. 
Experiments were performed with a Nd:glass based terawatt class laser system to characterize stimulated Raman forward scatter and stimulated Raman backscatter in underdense helium plasmas. Results of spectral analysis of near-forward scattered light and directly backscattered light are presented. These spectra are compared with predictions obtained from the dispersion relation. Observations of anti-Stokes features in the near-forward spectra are presented. Measurements of the energetic electrons commonly associated with the forward Raman instability are also presented and correlated with observations of the anti-Stokes features. The effect forward Raman scatter has on the propagation of a short pulse laser through an underdense plasma is also discussed.

Spectral observations of backscattered spectra are shown. These spectra contain both familiar and anomalous features. Severe modulations and a shift of spectral energy toward the blue (relative to where the dispersion relation predicts the spectra) were observed in the backscattered SRS spectra. Potential mechanisms for the modulations are postulated. Evidence for coupling between the forward Raman electron plasma wave and stimulated Brillouin ion acoustic wave at high incident laser intensities and plasma densities is presented as well. 


\section{Chapter 1}

\section{Introduction}

Laser plasma interactions have been widely studied for many years. Historically, this field has been of interest not only for the interesting basic plasma physics, but also for the relevance to numerous applications. One of the most well known applications of laser-plasma interactions is inertial confinement fusion (ICF). ([68],[79], $[88],[93],[96],[117],[123])$ Laser plasmas are also of interest to x-ray lasers $([1],[24],[25]$, [105]), laser based accelerators ([57],[58], [63],[116]), and the recently proposed fast ignitor fusion project [115]. The success of these applications depends critically on the various instabilities that can be driven in plasmas.

In ICF, efficient coupling of the laser energy into the plasma fuel is vital; generation of instabilities reduces this coupling efficiency. Instabilities, specifically those which produce Langmuir waves, can generate hot electrons which preheat the fusion fuel; this is detrimental in ICF where a large temperature gradient between the hot coronal plasma and the cold fusion fuel is necessary. Hot electrons are also detrimental in optical field ionized $\mathrm{x}$-ray laser schemes since these schemes require cold, collisionless plasmas. The propagation of a short pulse laser through a long scalelength underdense plasma is important for laser based accelerators and the fast ignitor. In fact, the fast ignitor project relies on the propagation of a laser pulse through an underdense preformed channel. The generation of instabilities as the laser propagates in this channel can scatter laser light and cause longitudinal and transverse breakup of the 
pulse, thereby reducing the amount of laser energy available at the critical surface for the production of energetic electrons.

Parametric instabilities that can occur in plasmas include stimulated Brillouin scattering, stimulated Raman scattering, two plasmon decay, ion acoustic decay, the oscillating two stream instability, and filamentation. The first four of these instabilities are decay instabilities; i.e., the incident pump wave ("parent") decays into traveling "daughter" waves. The oscillating two stream and filamentation (ponderomotive, thermal, and relativistic) instabilities, on the other hand, are purely growing instabilities in which the "parent" wave also decays into "daughter" waves, but the "daughter" wave representing the plasma disturbance remains stationary. Each instabilty obeys frequency and wavenumber matching conditions which express the conservation of total wave energy and total wave momentum, respectively. In laserplasma interactions, the laser couples with thermal modes present in the plasma to drive parametric instabilities.

In the last 25 years, theoretical laser plasma interaction studies have become very specialized and computer simulations have gained prominence as a useful tool. Parametric instabilities, particularly stimulated Raman scattering, two plasmon decay, and stimulated Brillouin scattering, have been a common subject for theoretical and computational investigations $([40],[51],[74])$. Publications have included plasma heating resulting from forward and backward Raman scattering ([47],[48]), two-dimensional studies of instabilities [2], nonlocal effects of stimulated Raman scattering [9], the production of hot electrons by two plasmon decay [121], and the effects of the plasma noise source on stimulated scattering [14].

$\mathrm{CO}_{2}$ lasers have been used to experimentally study some of these laser-plasma interactions. These lasers are frequently operated at $10.6 \mu \mathrm{m}$ and produce nanosecond pulses. Although $\mathrm{CO}_{2}$ lasers are typically only capable of reaching $10^{15}-10^{16} \mathrm{~W} / \mathrm{cm}^{2}$, the $I \lambda^{2}$ achievable can be quite large due to the long wavelength. Among the experiments performed with $\mathrm{CO}_{2}$ lasers are absorption measurements [7], stimulated Compton scattering observations ([71],[72]), and electron heating from two plasmon decay 
([42],[43]). Forward Raman scattering enhanced by backward Raman scattering has also been studied [120] and electrons accelerated via forward Raman scattering [56] have been measured. There have been many experiments studying parametric instabilities with glass based lasers $(1.05 \mu \mathrm{m})$ as well. While these systems can generate intensities similar to those achievable with $\mathrm{CO}_{2}$ lasers, the product $I \lambda^{2}$ is much less for glass lasers since the wavelength is shorter. Stimulated Raman scattering has been widely studied with glass lasers, both as a backscatter instability ([35],[41], [86],[101]) and a forward scatter instability ([11],[12],[70],[118]). Stimulated Brillouin scattering ([41], [130]), hot electron generation via two plasmon decay [121], and the competition between stimulated Raman scattering and stimulated Brillouin scattering have also been investigated [122].

Much of the theoretical work and computer simulations addressing laser-plasma interactions was, until recently, perfomed in a particular regime of laser and plasma parameters, one relevant to ICF and accessible experimentally with nanosecond laser technology. This regime is one of long ( $\tau \geq 100$ picoseconds), moderate intensity ( $\leq 10^{16} \mathrm{~W} / \mathrm{cm}^{2}$ ) laser pulses. In long pulse systems, the laser pulse length, $\mathrm{c} \tau$, is longer than both the Rayleigh range, $2 \mathrm{~L}_{\text {Rayleigh, }}$ and the plasma length, $\mathrm{L}_{\text {plasma }}$, as shown in figure 1.1(a). I.e.,

$$
c \tau>\mathrm{L}_{\text {plasma }} \geq 2 \mathrm{~L}_{\text {Rayleigh }}
$$

This regime will be referred to as the "long pulse regime" in the remainder of this work. In this regime, the temporal duration of the laser is longer than its transit time across the plasma. Therefore, the temporal growth of laser-plasma instabilities is limited by this transit time, not the laser pulse width. The interaction length is limited by the size of the plasma. It should be noted that theoretical investigations and computer simulations, while very useful in predicting plasma behavior, typically do not model experiments exactly. Approximations made to simplify the calculations often eliminate some physics associated with collisional damping mechanisms, long scalelengths, and kinetic effects.

Lasers commonly used to investigate the long pulse, moderate intensity laser 


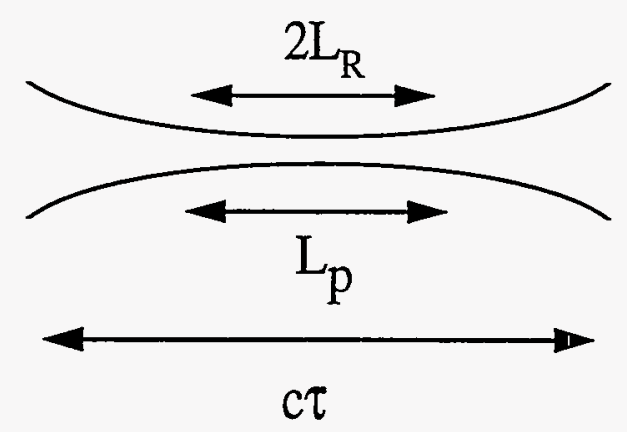

(a)

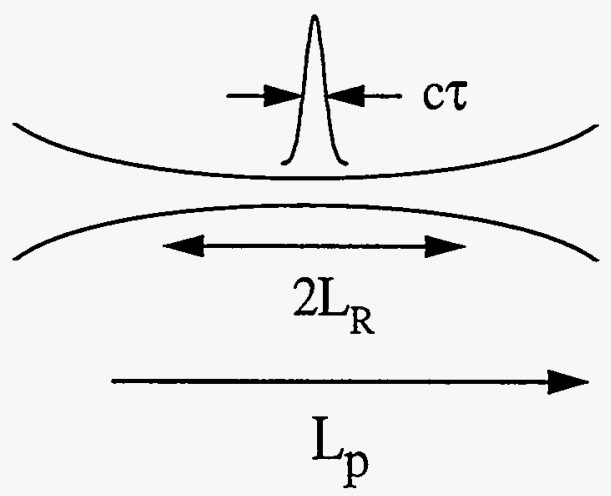

(b)

Figure 1.1: Illustration of relative sizes of laser pulse length, interaction regime, and plasma length for (a) the short pulse, high intensity laser regime (b) the long pulse, moderate intensity laser regime.

regime include glass based ( $\mathrm{Nd}$ :glass) and $\mathrm{CO}_{2}$ lasers. Table 1.1 shows typical parameters for a few of these lasers. Studies of instabilities in this long, moderate intensity laser pulse regime continue to be important in the ongoing investigation of ICF.

Advances in laser technology in the last several years have given rise to a new type of laser: the tabletop terawatt laser. These compact systems, capable of generating subpicosecond pulses with intensities as high as $10^{19} \mathrm{~W} / \mathrm{cm}^{2}$, have provided a new regime in which to study laser-plasma interactions. In this regime, the product $I \lambda^{2}$ is large. This results from the high laser intensity, in contrast to $\mathrm{CO}_{2}$ lasers, where the laser intensity is moderate but the laser wavelength is long. Some specific short pulse lasers are included in table 1.1. This new regime is distinctly different from the long pulse, moderate intensity regime (see figure 1.1). In this regime, shown in figure $1.1(\mathrm{~b})$,

$$
\mathrm{L}_{\text {plasma }} \geq 2 \mathrm{~L}_{\text {Rayleigh }}>\mathrm{c} \tau \text {. }
$$

In words, the pulse length of the laser is typically only a fraction of the Rayleigh range, and the plasma length can be larger than the Rayleigh range. Plasmas larger than the focal depth of the laser can be produced with short intense laser pulses 


\begin{tabular}{|c|c|c|c|c|c|}
\hline location & laser & type & wavelength & pulsewidth & $\begin{array}{r}\text { energy } \\
\text { (peak power) }\end{array}$ \\
\hline Livermore & Nova & $\begin{array}{l}\text { Nd:glass } \\
\text { (10 beams) }\end{array}$ & $\begin{array}{l}1.053 \mu \mathrm{m} \\
0.35,0.53 \mu \mathrm{m}\end{array}$ & $\begin{array}{l}200 \mathrm{psec} \\
\text { to } 5 \mathrm{nsec}\end{array}$ & $\begin{array}{l}6000 \mathrm{~J}(1 \omega)(30 \mathrm{TW}) \\
\text { (per beam) }\end{array}$ \\
\hline Rochester & Omega & $\begin{array}{l}\text { Nd:glass } \\
\text { (60 beams })\end{array}$ & $\begin{array}{l}1.053 \mu \mathrm{m} \\
0.35,0.53 \mu \mathrm{m}\end{array}$ & & $\begin{array}{l}500 \mathrm{~J}(1 \omega) \\
\text { (per beam) }\end{array}$ \\
\hline Los Alamos & Trident & $\begin{array}{l}\text { Nd:glass } \\
\text { ( } 3 \text { beams })\end{array}$ & $\begin{array}{l}0.53 \mu \mathrm{m} \\
1.053 \mu \mathrm{m}\end{array}$ & $\begin{array}{l}100 \mathrm{psec} \\
\text { to } 1 \mathrm{nsec}\end{array}$ & $\begin{array}{l}200 \mathrm{~J}(2 \mathrm{TW}) \\
\text { (per beam) }\end{array}$ \\
\hline $\begin{array}{l}\text { Ecole } \\
\text { Polytechnique }\end{array}$ & LULI & $\begin{array}{l}\text { Nd:glass } \\
\text { ( } 6 \text { beams })\end{array}$ & $\begin{array}{l}1.06 \mu \mathrm{m} \\
0.35,0.53 \mu \mathrm{m}\end{array}$ & 600 psec & $\begin{array}{l}100 \mathrm{~J} \\
(0.2 \mathrm{TW})\end{array}$ \\
\hline UCLA & & $\mathrm{CO}_{2}$ & $10.6 \mu \mathrm{m}$ & $300 \mathrm{psec}$ & $100 \mathrm{~J}(0.3 \mathrm{TW})$ \\
\hline \multirow[t]{2}{*}{ Livermore } & $10 \mathrm{TW}$ & Nd:glass & $1.053 \mu \mathrm{m}$ & 600 fsec & $6 \mathrm{~J}(10 \mathrm{TW})$ \\
\hline & LiSAF & $\mathrm{Cr}: \mathrm{LiSrAlF}_{6}$ & $825 \mathrm{~nm}$ & 125 fsec & $1 \mathrm{~J}(8 \mathrm{TW})$ \\
\hline UC Berkeley & & $\mathrm{Ti}: \mathrm{Al}_{2} \mathrm{O}_{3}$ & $807 \mathrm{~nm}$ & $60 \mathrm{fsec}$ & $0.23 \mathrm{~J}(3.8 \mathrm{TW})$ \\
\hline $\begin{array}{l}\text { U. Illinois } \\
\text { Chicago }\end{array}$ & & $\mathrm{KrF}$ & $248 \mathrm{~nm}$ & 500 fsec & $\begin{array}{l}0.15 \mathrm{~J} \\
(0.3 \mathrm{TW})\end{array}$ \\
\hline Rutherford & Vulcan & Nd:glass & $1.053 \mu \mathrm{m}$ & $800 \mathrm{fsec}$ & $25 \mathrm{~J}(31 \mathrm{TW})$ \\
\hline
\end{tabular}

Table 1.1: Parameters of some long and short pulse laser systems 
because ionization occurs early in the pulse. For example, for a 1 psec gaussian pulse with a peak intensity of $10^{18} \mathrm{~W} / \mathrm{cm}^{2}$, ionization can occur several picoseconds before the peak of the pulse. This implies that the high intensity portion of the laser pulse sees a preformed plasma. In fact, the saturation intensity for $\mathrm{He}^{2+}$ is reached more than 2 psec before the peak of the laser pulse for a 1 psec pulse of peak intensity $10^{18} \mathrm{~W} / \mathrm{cm}^{2}$. Therefore, when helium is used as the target gas, the high intensity portion of the laser pulse sees a uniform preformed plasma.

The scale length of laser-plasma interactions in this regime is dominated by the focal depth of the laser, not by the plasma size. Temporal growth of instabilities in this regime is limited by the temporal length of the laser pulse. As a result, for most instabilities, it is necessary to solve the spatio-temporal problem. Another consequence of short pulse lasers is that the laser pulses can be only a small number of plasma periods in duration. Since these short pulses have such high intensities, however, the growth rates for the instabilities are large and appreciable e-foldings can still occur. The high intensities associated with the laser pulses also lead to peak quiver velocities of electrons in the laser field, $\mathrm{v}_{\mathrm{osc}}=\mathrm{eE} / \mathrm{m} \omega_{0}$ (defined nonrelativistically), which approach or exceed the speed of light. Therefore, relativistic effects can not be neglected in these interactions. This new parameter regime will be referred to as the "short pulse regime" in the remainder of this work.

It is worth noting that recent progress in laser development has resulted in the amplification of very short ( $\leq 100 \mathrm{fsec}$ ) Ti:sapphire pulses to the multiterawatt level. ([131],[10],[113]) In fact, pulses as short as 26 femtoseconds have been amplified upto 2 TW. [131] These ultrashort, intense laser pulses could lead to more new and interesting investigations of laser plasma interactions.

Investigations in the last 10 years (theoretical, computational) have begun to reveal the peculiarities and interesting physics of short pulse, high intensity laser-plasma interactions. Because of their importance in laser based accelerators and other applications, two of the most common topics studied have been the propagation of short, intense laser pulses through plasmas and relativistic self focusing. Max's work in 
1974 on relativistic self focusing and relativistic self phase modulation [78], while not specifically a short pulse, high intensity laser calculation, was one of the early calculations done to study these relativistic effects. Subsequent publications investigating these phenomena in the short, intense laser pulse regime include works by Borisov ([16], [17],[19]), Chen [27], Mori [85], Sprangle ([106],[108]), Spatschek [109], and Sun [114]. Among the other effects that have been studied are nonlinear Thomson scattering $([46],[100])$, nonlinear depletion of short laser pulses [22]; and laser absorption in plasmas ([39],[124],[21],[52],[65], [61]). Parametric instabilities have also been investigated theoretically and computationally in the short pulse regime. Recent publications have examined the relationship between relativistic self focusing and stimulated Raman scattering ([3],[4]), the forward Raman and modulational instabilities ([80],[84]), and stimulated Raman backscatter ([99],[125]). Stimulated Brillouin scattering driven by a short pulse laser has also been investigated ([8],[54]).

While there have been numerous theoretical and simulation studies, few experimental observations of these laser-plasma interactions have been made. Some of these experimental observations are cited below. Self focusing experiments have included studies by Sullivan (ponderomotive) [112] and Borisov (relativistic) [18]. Electron temperature measurements have been made via Thomson scattering techniques by Offenberger [87] and with a retarding grid by Monot [82]. Stimulated Brillouin scattering in preformed plasmas, driven by a picosecond laser, has been investigated by Baldis [8] and Baton [13] while Darrow [34] observed strongly coupled stimulated Raman backscatter from a subpicosecond laser.

Stimulated Raman scattering is of particular interest in this newly accessible parameter regime. Unlike experiments performed with long pulse, moderate intensity lasers, stimulated Raman scattering is a dominant process in the short pulse, high intensity laser, underdense ( $\mathrm{n} \leq 0.03 \mathrm{n}_{\mathrm{cr}}$ ) plasma regime. Other instabilities, such as stimulated Brillouin scattering, typically do not achieve appreciable growth within the temporal duration of the laser at these densities. Raman scattering is also of interest because of its use as a density diagnostic ([28],[90]) and its relevance to 
optical field ionized x-ray laser schemes $([1],[44])$ and the recently proposed fast ignitor project.([115]) Recent particle-in-cell simulations have raised concerns about the effects Raman scattering will have on these applications. Simulations studying stimulated Raman backscatter have shown that, in this regime, background electrons can be heated to a level detrimental for recombination x-ray lasers [125]. Massively parallel simulations have found that, as a short, intense laser pulse propagates through an underdense plasma ([37], [38],[84]), forward Raman scatter is driven and longitudinal and transverse beam breakup occurs. This could seriously affect the fast ignitor and laser based accelerators.

The goal of the experiments described in this work was to characterize the interaction of short, high intensity laser pulses with underdense plasmas. Specifically, stimulated Raman scattering was studied to determine the behavior of this instability in the subpicosecond, high intensity laser underdense plasma regime. Stimulated Raman backscatter and forward scatter, the electrons associated with Raman forward scatter, and the effect of Raman forward scatter on the propagation of a short pulse laser through an underdense plasma were investigated. The results of this investigation are presented in the following chapters.

In chapter 2, the basic theory of stimulated Raman scattering is presented. Coupled partial differential equations are derived and solved to model the growth of stimulated Raman scattering. The derivation of the dispersion relation is also reviewed (nonrelativistically) and solved (relativistically and nonrelativistically) to predict the spectral properties of forward and backward stimulated Raman scatter. The terawatt laser system and experimental configurations used as well as plasma formation and characterization are discussed in chapter 3 . In chapter 4 , the results of experiments investigating forward Raman scatter, its associated hot electrons, and its affect on short pulse laser propagation are presented. Chapter 5 describes the spectral anamolies and reflectivity saturation observed in stimulated Raman backscatter experiments. The experimental spectra from both forward and backward stimulated Raman scatter are 
compared with the solution of the dispersion relation derived in chapter 2 . The summary and some general conclusions about stimulated Raman scatter in the short pulse laser regime are presented in chapter 6 . 


\section{Chapter 2}

\section{Theory of Stimulated Raman Scattering}

As discussed in chapter 1, stimulated Raman scattering is a laser-plasma instability relevant to several applications. In this chapter, Raman scattering is theoretically modeled to predict the behavior of the instability in the short pulse, high intensity laser, underdense plasma regime. First, the general one-dimensional description of stimulated Raman scattering is reviewed. The coupled partial differential equations are then derived and solved to illustrate the growth of Raman scattering. In this work, only the special case of an underdense, cold homogeneous plasma is considered as this closely approximates the experiments of chapters 4 and 5 . The dispersion relation is derived (nonrelativistic) and solved (nonrelativistic and relativistic) for both the backscattered and forward scattered problems. The growth rates for stimulated Raman scattering (backward and forward) are also derived. The formalism of Kruer [64] is followed in deriving coupled equations while the formalism of Forslund [51] is used in the derivation of the dispersion relation. 


\subsection{Basic theory of Stimulated Raman Scattering}

Stimulated Raman scatter (SRS) is a parametric decay instability observed in laser driven plasmas. In $\mathrm{SRS}$, an incident electromagnetic wave $\left(\omega_{0}, \mathrm{k}_{0}\right)$ resonantly decays into an electron plasma wave $\left(\omega_{\mathrm{e}}, \mathbf{k}_{\mathrm{e}}\right)$ and a scattered electromagntic wave $\left(\omega_{\mathrm{s}}, \mathbf{k}_{\mathrm{s}}\right)$, as depicted in figure 2.1(a). In this process, some of the incident energy is scattered and some is transferred to an electron plasma wave. The "feedback loop" describing how SRS grows is shown in figure 2.1(b). In words, an initial thermal density fluctuation,

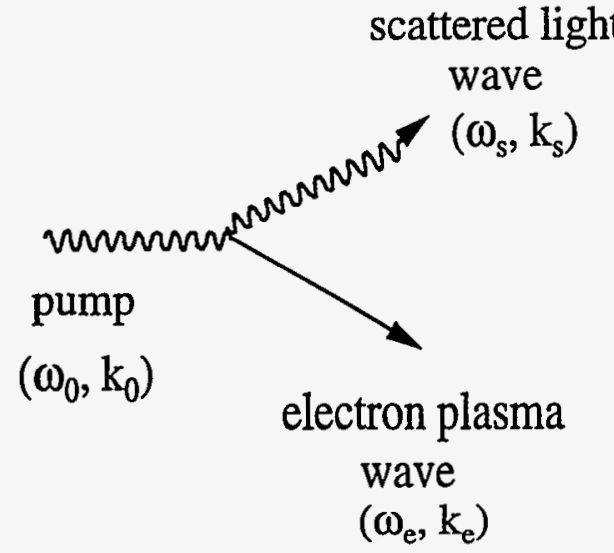

(a)

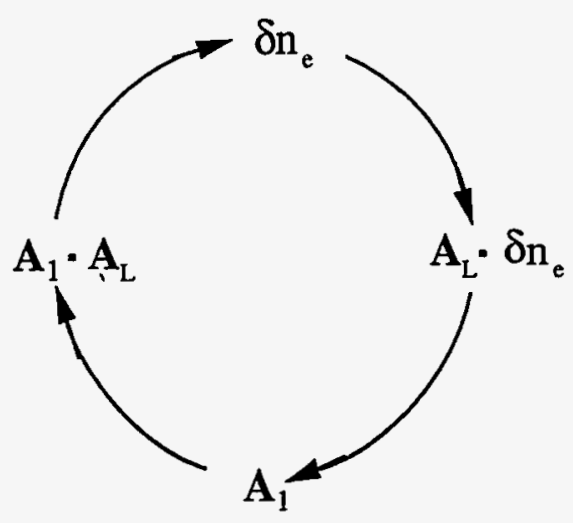

(b)

Figure 2.1: (a) Illustration of decay process of SRS and (b) schematic of the SRS "feedback" loop.

$\delta \mathrm{n}_{\mathrm{e}}$, is present in the plasma. An incident light wave (i.e., a laser of frequency $\omega_{0}$ ) causes electrons in the density fluctuation to oscillate transversly with velocity $\mathrm{v}_{\text {osc }}=$ e $A_{L} / m c$ where $A_{L}$ is the vector potential of the laser. If $\omega$ and $k$ are properly matched, these oscillating particles generate an oscillating current and reradiate a scattered light wave of frequency $\omega_{\mathrm{s}}$ and vector potential $\mathbf{A}_{\mathbf{1}}$. The vector potential of the scattered light wave interferes with the incident wave, resulting in a spatial variation in the ponderomotive pressure, $\mathbf{A}_{\mathbf{L}} \cdot \mathbf{A}_{\mathbf{1}}$. This pressure is in phase with and further enhances the density perturbation, $\delta \mathrm{n}_{\mathrm{e}}$, which subsequently increases the amplitude of the scattered light wave, $\mathbf{A}_{1}$, allowing the plasma and scattered light wave to grow unstable. This process is illustrated mathematically in the coupled 
partial differential equations derived in the section 2.2.

SRS, like other parametric instabilities, obeys frequency and wavenumber matching conditions, which are given by

$$
\begin{aligned}
& \omega_{0}=\omega_{\mathrm{s}} \pm \mathrm{m} \omega_{\mathrm{e}} \\
& \mathrm{k}_{0}=\mathrm{k}_{\mathrm{s}} \pm m \mathrm{k}_{\mathrm{e}}
\end{aligned}
$$

where $\mathrm{m}$ is an integer. The $+(-)$ refers to the $\mathrm{mth}$ order "Stokes" ("anti-Stokes") mode which is frequency or wavenumber down-shifted (upshifted) by $\mathrm{m}$ times the electron plasma wave frequency and wavenumber. For $\mathrm{m} \geq 1$, this results in the classical cascaded spectrum. [37] Physically, this means that the density fluctuation which is unstable is that which is in phase with the spatially and temporally periodic ponderomotive pressure. The matching conditions dictate where $\left(\omega_{\mathbf{s}}\right)$ and in what direction $\left(\mathbf{k}_{\mathbf{s}}\right)$ the scattered light will be observable. Backscattered SRS is a three wave process so the appropriate matching condition is given by $\mathrm{m}=1$. In contrast, forward SRS can be a many wave process $(\mathrm{m}>1)$. Figure 2.2 illustrates the $\mathrm{k}$ matching conditions for directly backscattered SRS (3-wave) and forward scattered SRS (4-wave). For backscattered SRS, the electron plasma wave travels in the same

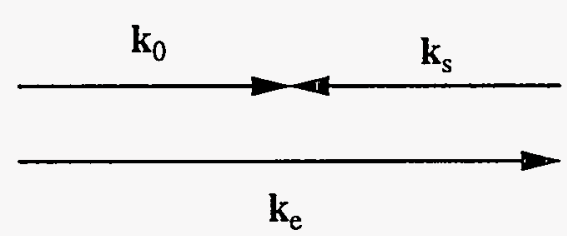

(a)

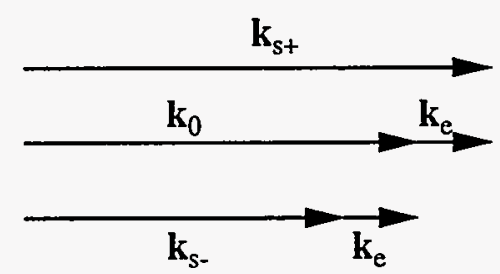

(b)

Figure 2.2: Wavenumber matching (k-matching) for (a) stimulated Raman backscatter and (b) stimulated Raman forward scatter

direction as the incident wave while the scattered light wave propagates in the opposite direction. In forward SRS, all the waves propagate in the forward direction. In vector 
notation, defining $\mathrm{k}_{0}$ as $\mathrm{k}_{0} \hat{\mathrm{x}}, \mathrm{k}_{\mathrm{s}}=-\mathrm{k}_{\mathrm{s}} \hat{\mathrm{x}}$ and $\mathrm{k}_{\mathrm{e}}=\mathrm{k}_{\mathrm{e}} \hat{\mathrm{x}}$ for direct backscatter while $k_{s}=k_{s} \hat{x}$ and $k_{e}=k_{e} \hat{x}$ for direct forward scatter. For backscattered SRS, only the Stokes mode is resonant (see section 2.3.1) so the wavenumber matching condition becomes $k_{0}=-k_{s}+k_{e}$ The wavenumber matching condition for forward SRS is given by $k_{0}=k_{s} \pm m k_{e}$. Observation of the scattered light wave (back or foward scatter) provides clear evidence that stimulated Raman scattering is being driven in a plasma.

Each wave involved in SRS obeys a dispersion relation. These relations are given by

$$
\begin{aligned}
& \omega_{0}^{2}=\mathrm{k}_{0}{ }^{2} \mathrm{c}^{2}+\omega_{\mathrm{pe}}{ }^{2} \\
& \omega_{\mathrm{s}}^{2}=\mathrm{k}_{\mathrm{s}}{ }^{2} \mathrm{c}^{2}+\omega_{\mathrm{pe}}{ }^{2} \\
& \omega_{\mathrm{e}}{ }^{2}={\omega_{\mathrm{pe}}}^{2}+3 \mathrm{k}_{\mathrm{e}}{ }^{2} \mathrm{v}_{\mathrm{th}}{ }^{2}
\end{aligned}
$$

where the plasma frequency, $\omega_{\mathrm{pe}}$, is given by

$$
\omega_{\mathrm{pe}}=\sqrt{\frac{4 \pi \mathrm{n}_{\mathrm{o}} \mathrm{e}^{2}}{\mathrm{~m}_{\mathrm{e}}}} .
$$

Equation (2.5) is commonly known as the Bohm-Gross dispersion relation [15]. These dispersion relations govern the incident and scattered light waves and the electron plasma wave and are derived from the undriven $\left(\mathrm{v}_{\text {osc }} \rightarrow 0\right.$ ) coupled differential equations. They represent the normal modes of the plasma. Introduction of an electromagnetic laser field into the plasma leads to coupling of the normal modes and a dispersion relation which includes this coupling must be used. This is the dispersion relation derived and solved later in this chapter.

The parameter regime in which SRS can grow is easily obtained from the dispersion relations and the frequency matching condition. Equations (2.3) and (2.4) produce the relations $\omega_{0} \geq \omega_{\mathrm{pe}}$ and $\omega_{\mathrm{s}} \geq \omega_{\mathrm{pe}}$ respectively. Using the matching condition, (2.1), these inequalities can be combined to give $\omega_{0} \geq 2 \omega_{\mathrm{pe}}$. Since the plasma frequency is proportional to the square root of the density, the expression can be rewritten as $\mathrm{n}_{\mathrm{cr}} \geq 4 \mathrm{n}_{0}$, or

$$
\mathrm{n}_{0} \leq \frac{1}{4} \mathrm{n}_{\mathrm{cr}}
$$


Specifically, in order for SRS to grow, the plasma density must be less than one-fourth the critical density.

In order for SRS, or any other instability, to grow, the growth rate of the wave must exceed the damping rate. The growth rates for SRS backscatter and forward scatter in the abscence of damping will be derived later in this chapter. The "instability threshold" for stimulated Raman scattering is given by [64]

$$
\gamma_{0}=\sqrt{\gamma_{\mathrm{e}} \gamma_{\mathrm{s}}}
$$

where $\gamma_{0}$ is the growth rate of SRS in the absence of damping and $\gamma_{e}$ and $\gamma_{s}$ are the amplitude damping rates for the electron plasma wave and scattered light wave, respectively. These damping rates typically result from the presence of collisional damping or Landau damping. [64] Other factors affecting the threshold intensity necessary to drive stimulated Raman scattering include gradients in the plasma density and plasma inhomogeneity. [98] In this work, the discussion is confined to homogeneous plasmas. As an example, consider backscattered SRS with $\omega_{\mathrm{pe}} \ll \omega_{0}$ and only collisional damping. The growth rate for stimulated Raman backscatter in this limit is given by $\gamma_{0}=\frac{1}{2}\left(\mathrm{v}_{\mathrm{osc}} / \mathrm{c}\right) \sqrt{\omega_{\mathrm{pe}} \omega_{0}}$, where $\mathrm{v}_{\mathrm{osc}}$ is the oscillation velocity of an electron in the presence of the laser field $\left(\mathrm{v}_{\mathrm{osc}}=\mathrm{eE} / \mathrm{m} \omega\right)$. The collisional damping rates for the scattered light wave and the electron plasma wave are given by $\gamma_{\mathrm{s}}=\frac{1}{2} \omega_{\mathrm{pe}} / \omega_{\mathrm{s}}{ }^{2} \nu_{\mathrm{ei}}$ and $\gamma_{\mathrm{e}}=\frac{1}{2} \nu_{\mathrm{ei}}$, respectively, where $\nu_{\mathrm{ei}}$ is the electron-ion collision rate.[64] Substitution of $\gamma_{0}, \gamma_{\mathrm{s}}$, and $\gamma_{\mathrm{e}}$ into equation (2.7) yields

$$
\left(\frac{\mathrm{v}_{\mathrm{osc}}}{\mathrm{c}}\right)^{2} \geq \nu_{\mathrm{ei}}{ }^{2}\left(\frac{\omega_{\mathrm{pe}}}{\omega_{0}}\right)^{2}\left(\frac{1}{\omega_{\mathrm{pe}} \omega_{0}}\right)
$$

This corresponding intensity threshold can be very low. For example, if $\omega_{\mathrm{pe}}=\frac{1}{30} \omega_{0}$ and $\nu_{\mathrm{ei}}=5.4 \times 10^{10} \mathrm{sec}^{-1}$, i.e., typical of the experimental parameters, the threshold $\mathrm{v}_{\mathrm{osc}} / \mathrm{c}$ is $5.5 \times 10^{-6}$. Inclusion of Landau damping raises this threshold considerably, although if the plasma is assumed to be cold, the threshold oscillation velocity is still a small fraction of $\mathrm{c}$ for these parameters.

As stated earlier in this section, in stimulated Raman scattering, part of the incident energy is scattered and part is transferred to the electron plasma wave. The 
energy in the plasma wave can be transferred to electrons in the plasma via wavebreaking or Landau damping for backscatter SRS and by wavebreaking for forward SRS. Since the electron plasma wave can have a high phase velocity $\left(v_{p h} \sim c\right)$, energetic electrons are produced when the plasma wave damps or wavebreaks. This results in a so-called heated tail in the electron distribution. For stimulated Raman backscatter, $\mathrm{v}_{\mathrm{ph}} \approx \frac{1}{2} \mathrm{c}\left(\omega_{\mathrm{pe}} / \omega_{0}\right) \ll \mathrm{c}$ for an underdense plasma. The "rule of thumb" for estimating the temperature of the heated electron tail in this case is [64]

$$
\Theta_{\mathrm{hot}} \approx \frac{1}{2} \mathrm{mv}_{\mathrm{ph}}^{2} .
$$

For a plasma density, $\mathrm{n}$, of $0.01 \mathrm{n}_{\mathrm{cr}}$, the phase velocity is $\mathrm{v}_{\mathrm{ph}}=0.05 \mathrm{c}$, which gives an electron temperature of approximately $1 \mathrm{keV}$.

For forward SRS, the phase velocity of the electron plasma wave can approach $c$, so a relativistically correct expression valid for a fast wave must be used to estimate the temperature of the heated electron tail. The maximum energy electrons trapped in a plasma wave can gain is given by

$$
\mathrm{W}_{\max }=2 \epsilon \gamma_{\mathrm{ph}}{ }^{2} \mathrm{mc}^{2}
$$

where $\epsilon=\mathrm{eE} / \mathrm{mc} \omega_{\mathrm{pe}}$ is the normalized amplitude of the plasma wave, and $\gamma_{\mathrm{ph}}{ }^{2}=$ $1 /\left(1-\mathrm{v}_{\mathrm{ph}}{ }^{2} / \mathrm{c}^{2}\right)$. ([37],[116]) For forward SRS, let $\mathrm{v}_{\mathrm{ph}}=\mathrm{v}_{\mathrm{g}}=\mathrm{c} \sqrt{1-\omega_{\mathrm{pe}}{ }^{2} / \omega_{0}^{2}}$ where $\mathrm{v}_{\mathrm{g}}$ is the group velocity of the plasma wave. This gives $\gamma_{\mathrm{ph}}{ }^{2}=\omega_{0}^{2} / \omega_{\mathrm{pe}}{ }^{2}$. Therefore, $\mathrm{W}_{\max }=2 \epsilon\left(\omega_{0}^{2} / \omega_{\mathrm{pe}}{ }^{2}\right) \mathrm{mc}^{2}$. If $\epsilon=0.1$ and $\omega_{\mathrm{pe}}=\frac{1}{10} \omega_{0}$, the maximum energy of an accelerated electron would be approximately $10 \mathrm{MeV}$. Clearly, extremely energetic electrons are possible as $\mathrm{v}_{\mathrm{ph}}$ approaches $\mathrm{c}$, as is the case for forward scattered electron plasma waves with $\mathrm{n} \ll \mathrm{n}_{\mathrm{cr}}$. In fact, such energetic electrons have been observed in long pulse experiments and attributed to forward stimulated Raman scattering. ([56],[118]) The generation of energetic electrons, and the resulting heating of the plasma, is detrimental for ICF and optical field ionized recombination x-ray laser schemes, as discussed in chapter 1.

The remainder of this chapter is devoted to the derivation and solution of the coupled equations and the dispersion relation. The coupled equations are a set of 
partial differential equations which describe the evolution of the waves involved in the SRS process. When solving the coupled equations, the slowly varying envelope approximation is used and the amplitudes of the envelopes of the scattered light and electron plasma waves are generated (as a function of space and time). These amplitudes can be used to determine the growth of the waves as a laser propagates through a plasma. The normal mode dispersion relations (equations (2.3-2.5)) can be obtained from the coupled equations. While the coupled equations can be used to model both forward and backward SRS, only the solution to the backscatter problem will be discussed here.

The dispersion relation is a driven equation which describes the relationship between the frequency and wavenumber of the plasma wave. This equation is derived from the coupled equations by writing the light wave, $A_{L}$ as an envelope times an oscillation, then Fourier analyzing the coupled equations. The solution to the dispersion relation can be used to predict the spectral shape and location of the SRS features. In this work, the nonrelativistic and relativistic versions of this dispersion relation are solved for both stimulated Raman backscatter and stimulated Raman forward scatter. These solutions can then be compared with experimental spectra. The coupled equations and the dispersion relation are obtained using the fluid equations. Since the fluid equations as shown in section 2.2 neglect damping, they are only valid when $\mathrm{k} \lambda_{\text {debye }}<0.3$ where kinetic effects are unimportant. [62] Therefore, the coupled equations and dispersion relation, as derived in sections 2.2 and 2.3, are only appropriate when $k \lambda_{\text {debye }}<0.3$. The product $k \lambda_{\text {debye }}$ represents the ratio of the thermal velocity of the plasma, $\mathrm{v}_{\mathrm{th}}$, to the phase velocity of the plasma wave, $\mathrm{v}_{\mathrm{ph}}$. When $v_{\text {th }}$ is on the order of $v_{p h}$ (i.e., $k \lambda_{\text {debye }}>0.3$ ), electrons can be accelerated and decelerated by the plasma wave. This indicates that wave-particle interactions are important and a kinetic treatment must be used to describe the interactions. For $\mathrm{k} \lambda_{\text {debye }}<0.3$, energy is not efficiently transferred between waves and the bulk of the electrons in the thermal distribution and a fluid treatment is sufficient. 


\subsection{The Coupled Equations}

The equations used in this work to describe plasmas are Maxwell's equations and the fluid equations. The most complete description of a plasma is given by Boltzmann's equation, which includes kinetic effects and collisions. Vlasov's equation, which is also known as the collisionless Boltzmann equation, includes kinetic effects, but not collisions. For the parameter regime of this work, $\mathrm{k} \lambda_{\text {debye }}<0.3\left(\nu_{\mathrm{ei}} / \omega_{\mathrm{pe}} \approx 10^{-4} \ll 1\right)$ so the fluid equations provide a sufficient description (see section 2.1). In this work, the plasma is assumed to be cold and homogeneous unless otherwise noted.

The coupled equations, which are differential equations that describe electron density, scattered field, and incident field perturbations, can be derived from Maxwell's equations and the fluid equations. This derivation ([40],[51]) is reviewed here, following the formalism of Kruer [64]. For an unmagnetized medium, Maxwell's equations are given by:

$$
\begin{aligned}
\nabla \cdot \mathbf{E} & =4 \pi \rho \\
\nabla \cdot \mathbf{B} & =0 \\
\nabla \times \mathbf{E} & =-\frac{1}{\mathrm{c}} \frac{\partial \mathbf{B}}{\partial \mathrm{t}} \\
\nabla \times \mathbf{B} & =\frac{4 \pi}{\mathrm{c}} \mathbf{J}+\frac{1}{\mathrm{c}} \frac{\partial \mathbf{E}}{\partial \mathrm{t}}
\end{aligned}
$$

in Gaussian units. The fluid equations, which are obtained by taking the moments of Vlasov's equation, are given by:

$$
\begin{aligned}
& \frac{\partial \mathrm{n}_{\alpha}}{\partial \mathrm{t}}+\nabla \cdot \mathrm{n}_{\alpha} \mathrm{v}_{\alpha}=0 \\
& \mathrm{n}_{\alpha} \mathrm{m}_{\alpha}\left(\frac{\partial}{\partial \mathrm{t}}+\mathrm{v}_{\alpha} \cdot \nabla\right) \mathrm{v}_{\alpha}=\mathrm{n}_{\alpha} \mathrm{q}_{\alpha}\left(\mathrm{E}+\frac{\mathrm{v}_{\alpha} \times \mathrm{B}}{\mathrm{c}}\right)-\nabla \mathrm{p}_{\alpha}
\end{aligned}
$$

where

$$
\begin{aligned}
& \mathbf{J}=\sum_{\alpha} \mathrm{n}_{\alpha} \mathrm{q}_{\alpha} \mathrm{v}_{\alpha} \\
& \rho=\sum_{\alpha} \mathrm{n}_{\alpha} \mathrm{q}_{\alpha}
\end{aligned}
$$


and $\mathrm{n}$ is the density, $\mathrm{m}$ is the mass, $\mathrm{v}$ is the velocity, $\mathrm{q}$ is the charge, $\mathrm{p}$ is the pressure, and the subscript $\alpha$ denotes the species. Both collisions and damping are neglected in these fluid equations. Equation (2.15) is known as the continuity equation and equation (2.16) is the momentum transport equation. Typically, only two species are present, electrons $(\alpha=e)$ and a single ion species $(\alpha=\mathrm{i})$. In this work, the ions are assumed to be massive $\left(\mathrm{m}_{\mathrm{i}} \gg \mathrm{m}_{\mathrm{e}}\right)$ and immobile compared to the electrons. I.e., the ion acoustic velocity, $c_{s}$, is much less than the thermal velocity of the electrons, $\mathrm{v}_{\mathrm{th}}$. Therefore, the current density, $\mathbf{J}$, is produced solely by the electrons. The total charge density, $\rho$, has both electron and ion contributions.

The pressure gradient term, $\nabla \mathrm{p}$, in equation (2.16) can be found using

$$
\mathrm{pn}^{-\Gamma}=\text { constant }
$$

Writing the pressure and density as the sum of a uniform background plus a small fluctuation, $\mathrm{p}=\mathrm{p}_{0}+\mathrm{p}_{1}$ and $\mathrm{n}=\mathrm{n}_{0}+\mathrm{n}_{1}$, and plugging into (2.19) yields an expression for the pressure fluctuation, $\mathrm{p}_{1}$.

$$
\mathrm{p}_{1}=\mathrm{p}_{0} \Gamma \frac{\mathrm{n}_{1}}{\mathrm{n}_{0}}
$$

The gradient of $\mathrm{p}$ is then

$$
\begin{aligned}
\nabla \mathrm{p} & =\nabla\left(\mathrm{p}_{0}+\mathrm{p}_{1}\right) \\
& =\nabla \mathrm{p}_{1} \\
& =\mathrm{kT} \nabla \mathrm{n}_{1}
\end{aligned}
$$

where the ideal gas law, $\mathrm{p}=\mathrm{nkT}$, was used. Using equation (2.21), the momentum equation, (2.16), can be written as

$$
\mathrm{n}_{\alpha} \mathrm{m}_{\alpha}\left(\frac{\partial}{\partial \mathrm{t}}+\mathrm{v}_{\alpha} \cdot \nabla\right) \mathrm{v}_{\alpha}=\mathrm{n}_{\alpha} \mathrm{q}_{\alpha}\left(\mathrm{E}+\frac{\mathrm{v}_{\alpha} \times \mathbf{B}}{\mathrm{c}}\right)-\mathrm{kT} \Gamma \nabla \mathrm{n}_{1 \alpha}
$$

To obtain the coupled equations, Ampere's law (equation (2.14)) and Gauss' law (equation (2.11) must first be written in terms of the vector potential $\mathbf{A}$ and the electric potential $\phi$. The electric and magnetic fields, expressed in terms of $\mathbf{A}$ and $\phi$, 
are given by

$$
\begin{aligned}
& \mathbf{B}=\nabla \times \mathbf{A} \\
& \mathbf{E}=-\frac{1}{\mathrm{c}} \frac{\partial \mathbf{A}}{\partial \mathrm{t}}-\nabla \phi .
\end{aligned}
$$

Using the Coulomb gauge, $\nabla \cdot \mathbf{A}=0$, and the vector identity

$$
\nabla \times(\nabla \times \mathbf{w})=\nabla(\nabla \cdot \mathbf{w})-\nabla^{2} \mathbf{w}
$$

equation (2.14) becomes

$$
\nabla^{2} \mathbf{A}-\frac{1}{c^{2}} \frac{\partial^{2} \mathbf{A}}{\partial \mathrm{t}^{2}}=-\frac{4 \pi}{\mathrm{c}} \mathbf{J}+\frac{1}{\mathrm{c}} \frac{\partial}{\partial \mathrm{t}}(\nabla \phi)
$$

and equation (2.11) becomes

$$
\nabla^{2} \phi=-4 \pi \rho .
$$

To evaluate $\frac{\partial}{\partial t}(\nabla \phi)$, take the partial derivative with respect to $t, \frac{\partial}{\partial t}$, of equation (2.27) and use the conservation of charge, which is given by

$$
\frac{\partial \rho}{\partial \mathrm{t}}+\nabla \cdot \mathbf{J}=0
$$

to obtain

$$
\begin{aligned}
\frac{\partial}{\partial \mathrm{t}}\left(\nabla^{2} \phi\right) & =-4 \pi \frac{\partial \rho}{\partial \mathrm{t}} \\
& =4 \pi \nabla \cdot \mathbf{J}
\end{aligned}
$$

The current density, $\mathbf{J}$, can be written as the sum of a transverse component, $\mathbf{J}_{t}$, associated with the light waves, and a longitudinal component, $\mathbf{J}_{1}$, associated with the electrostatic plasma wave, $\mathbf{J}=\mathbf{J}_{\mathbf{t}}+\mathbf{J}_{\mathbf{l}}$. The transverse current density results from the oscillation of electrons in the laser field. Therefore, by definition, $\mathbf{J}_{t}$ is in the same direction as the vector potential $\mathbf{A}$. Since the Coulomb gauge was chosen $(\nabla \cdot \mathbf{A}=0), \nabla \cdot \mathbf{J}_{\mathrm{t}}=0$. Equation (2.29) can then be rewritten as

$$
\nabla \cdot\left(\frac{\partial}{\partial t}(\nabla \phi)-4 \pi \mathbf{J}_{1}\right)=0
$$


which leads to

$$
\frac{\partial}{\partial \mathrm{t}}(\nabla \phi)=4 \pi \mathbf{J}_{\mathbf{l}}
$$

Substituting this expression into equation (2.26) yields

$$
\left(\frac{1}{c^{2}} \frac{\partial^{2}}{\partial \mathrm{t}}-\nabla^{2}\right) \mathbf{A}=\frac{4 \pi}{\mathrm{c}} \mathbf{J}_{\mathrm{t}} .
$$

The transverse current density, $\mathbf{J}_{t}$, can be written as $\mathbf{J}_{t}=-n_{e}$ eu $u_{t}$, where $u_{t}$ is the oscillation velocity of an electron in the electric field of the laser, $\mathbf{E}$. This oscillation velocity, $\mathrm{u}_{\mathrm{t}}$, can be found using the Lorentz force equation.

$$
\begin{aligned}
\frac{\mathrm{d} \mathbf{u}_{\mathrm{t}}}{\mathrm{dt}} & =-\frac{\mathrm{e}}{\mathrm{m}} \mathbf{E}_{\mathrm{t}} \\
& =\frac{\mathrm{e}}{\mathrm{mc}} \frac{\partial \mathbf{A}}{\partial \mathrm{t}} \\
\mathbf{u}_{\mathrm{t}} & =\frac{\mathrm{eA}}{\mathrm{mc}}=\mathrm{v}_{\mathrm{osc}}
\end{aligned}
$$

This is simply the nonrelativistic expression for the oscillation velocity of an electron in the presence of an electromagnetic field, $v_{o s c}$. Substitution of $\mathbf{J}_{\mathbf{t}}$ into equation (2.32) gives

$$
\begin{aligned}
\left(\frac{1}{c^{2}} \frac{\partial^{2}}{\partial t^{2}}-\nabla^{2}\right) \mathbf{A} & =-\frac{4 \pi \mathrm{e}^{2}}{\mathrm{mc}^{2}} n_{\mathrm{e}} \mathbf{A} \\
& =-\frac{\omega_{\mathrm{pe}}{ }^{2}}{\mathrm{c}^{2} \mathrm{n}_{0}} \mathrm{n}_{\mathrm{e}} \mathbf{A}
\end{aligned}
$$

where $n_{0}$ is the uniform background plasma density. The quantities $n_{e}$ and $\mathbf{A}$ can be written as

$$
\begin{aligned}
& \mathbf{A}=\mathbf{A}_{\mathrm{L}}+\mathbf{A}_{1} \\
& \mathrm{n}_{\mathrm{e}}=\mathrm{n}_{0}+\mathrm{n}_{1}
\end{aligned}
$$

where $\mathbf{A}_{\mathrm{L}}$ is the incident light wave, $\mathbf{A}_{1}$ is the scattered light wave, and $\mathrm{n}_{1}$ is a small amplitude density fluctuation (i.e., $\delta \mathrm{n}_{\mathrm{e}}$ ). Substituting equations (2.35) and (2.36) into equation (2.34) to linearize the equation yields

$$
\left(\frac{\partial^{2}}{\partial \mathrm{t}^{2}}-\mathrm{c}^{2} \nabla^{2}\right)\left(\mathbf{A}_{\mathrm{L}}+\mathbf{A}_{1}\right)=-\frac{\omega_{\mathrm{pe}}^{2}}{\mathrm{n}_{0}}\left(\mathrm{n}_{0}+\mathrm{n}_{1}\right)\left(\mathbf{A}_{\mathrm{L}}+\mathbf{A}_{1}\right)
$$


This equation provides two related equations: one describing the depletion of the incident pump wave, $\mathbf{A}_{\mathrm{L}}$, and one describing the amplitude of the scattered light wave, $\mathbf{A}_{1}$. The pump depletion equation is given by

$$
\left(\frac{\partial^{2}}{\partial \mathrm{t}^{2}}-\mathrm{c}^{2} \nabla^{2}+\omega_{\mathrm{pe}}^{2}\right) \mathbf{A}_{\mathrm{L}}=-\frac{\omega_{\mathrm{pe}}{ }^{2}}{\mathrm{n}_{0}} \mathrm{n}_{1} \mathbf{A}_{1}
$$

and the scattered wave equation is given by

$$
\left(\frac{\partial^{2}}{\partial \mathrm{t}^{2}}-\mathrm{c}^{2} \nabla^{2}+\omega_{\mathrm{pe}}{ }^{2}\right) \mathbf{A}_{1}=-\frac{\omega_{\mathrm{pe}}{ }^{2}}{\mathrm{n}_{0}} \mathrm{n}_{1} \mathbf{A}_{\mathrm{L}}
$$

These two equations, (2.38) and (2.39), are two of the so-called coupled equations which describe stimulated Raman scattering. The dispersion relations for the incident and scattered light waves (equations (2.3) and (2.4) in section 2.1) are readily obtained from these coupled equations. Neglecting the coupling because $n_{1} A_{L}$ and $n_{1} A_{1}$ are second order terms, the right hand side of equations (2.38) and (2.39) are both equal to zero. Fourier analyzying (i.e., $\frac{\partial}{\partial t} \rightarrow i \omega, \nabla \rightarrow-i k$ ) gives

$$
\left(-\omega_{0}^{2}+c^{2} k_{0}^{2}+\omega_{p e}^{2}\right) \mathbf{A}_{L}=0
$$

for equation (2.38) and

$$
\left(-\omega_{\mathrm{s}}{ }^{2}+\mathrm{c}^{2} \mathbf{k}_{\mathrm{s}}{ }^{2}+\omega_{\mathrm{pe}}{ }^{2}\right) \mathbf{A}_{1}=0
$$

for equation (2.39). Assuming $\mathbf{A}_{\mathrm{L}} \neq 0$ and $\mathbf{A}_{1} \neq 0$, the undriven dispersion relations from section 2.1 are obtained.

$$
\begin{aligned}
& \omega_{0}{ }^{2}=\mathrm{k}_{0}{ }^{2} \mathrm{c}^{2}+\omega_{\mathrm{pe}}{ }^{2} \\
& \omega_{\mathrm{s}}{ }^{2}=\mathrm{k}_{\mathrm{s}}{ }^{2} \mathrm{c}^{2}+\omega_{\mathrm{pe}}{ }^{2}
\end{aligned}
$$

For parameters relevant to this work, the pump, $\mathbf{A}_{\mathrm{L}}$, is large $\left(\mathrm{v}_{\text {osc }} / \mathrm{c} \approx 1\right)$ and will be assumed to not be appreciably depleted during the growth of the SRS. When more than $5 \%$ of the incident laser energy is lost, pump depletion can change the dynamics of the growth and equation (2.38) can not be neglected in the analysis. In this work, less than $5 \%$ of the energy is lost for backscattered SRS (see section 5.3.3). Therefore, 
equation (2.38) will be neglected when the coupled equations are solved for stimulated Raman backscatter later in this section.

To obtain the differential equation describing the amplitude of the density fluctuation, $\mathrm{n}_{1}$, the momentum fluid equation, (2.22), and the continuity equation, (2.15), specialized for electrons, are used. The electron velocity, $v_{e}$, can be written as the sum of longitudinal and transverse components,

$$
\mathrm{v}_{\mathrm{e}}=\mathrm{u}+\frac{\mathrm{e}}{\mathrm{mc}} \mathbf{A}
$$

where the transverse part of the electron velocity is written as the oscillation velocity, $\mathbf{u}_{\mathrm{t}}=\mathrm{eA} / \mathrm{mc}$ (see equation (2.33)). The subscript ' $\mathrm{e}$ ' on the mass will be dropped in the following equations since the electron mass is the only mass present. Substitution of equation (2.40), along with equations (2.23) and (2.24), into equation (2.22) yields

$$
\begin{aligned}
\frac{\partial \mathrm{u}}{\partial \mathrm{t}}= & \frac{\mathrm{e}}{\mathrm{m}} \nabla \phi-\frac{\mathrm{kT} \gamma}{\mathrm{n}_{\mathrm{e}} \mathrm{m}} \nabla \mathrm{n}_{1}-\left\{\left(\mathbf{u}+\frac{\mathrm{e}}{\mathrm{mc}} \mathbf{A}\right) \times\left(\nabla \times \frac{\mathrm{e}}{\mathrm{mc}} \mathbf{A}\right)\right. \\
& \left.+\left(\mathbf{u}+\frac{\mathrm{e}}{\mathrm{mc}} \mathbf{A}\right) \cdot \nabla\left(\mathbf{u}+\frac{\mathrm{e}}{\mathrm{mc}} \mathbf{A}\right)\right\}
\end{aligned}
$$

Use of the vector identity

$$
2(\mathbf{w} \cdot \nabla) \mathbf{w}=\nabla(\mathbf{w} \cdot \mathbf{w})-2(\mathbf{w} \times(\nabla \times \mathbf{w}))
$$

reduces equation (2.41) to

$$
\frac{\partial \mathrm{u}}{\partial \mathrm{t}}=\frac{\mathrm{e}}{\mathrm{m}} \nabla \phi-\frac{1}{2} \nabla\left(\mathbf{u}+\frac{\mathrm{e}}{\mathrm{mc}} \mathbf{A}\right)^{2}-\frac{\mathrm{kT} \Gamma}{\mathrm{n}_{\mathrm{e}} \mathrm{m}} \nabla \mathrm{n}_{1}
$$

The third term on the right hand side of this equation can be simplified and rewritten as

$$
\frac{\mathrm{kT} \Gamma}{\mathrm{n}_{\mathrm{e}} \mathrm{m}} \nabla \mathrm{n}_{1}=3 \mathrm{v}_{\mathrm{th}}{ }^{2} \frac{\nabla \mathrm{n}_{1}}{\mathrm{n}_{\mathrm{e}}}
$$

since $\Gamma=3$ for this work (adiabatic system) and $\mathrm{v}_{\mathrm{th}}{ }^{2}=\mathrm{kT} / \mathrm{m}$, the thermal velocity of the electrons. Using equations (2.35) and (2.36) to linearize equations (2.43) and (2.15), assuming that the longitudinal velocity, $u$, is a small fluctuation, keeping only first order fluctuations (i.e., neglecting terms which are second order and higher) and 
assuming $\mathrm{n}_{1}<<\mathrm{n}_{0}$, gives

$$
\begin{aligned}
\frac{\partial \mathrm{u}}{\partial \mathrm{t}} & =\frac{\mathrm{e}}{\mathrm{m}} \nabla \phi-\frac{1}{2} \nabla\left(\mathrm{u}+\frac{\mathrm{e}}{\mathrm{mc}}\left(\mathbf{A}_{\mathrm{L}}+\mathbf{A}_{1}\right)\right)^{2}-3 \mathrm{v}_{\mathrm{th}}{ }^{2} \frac{\nabla \mathrm{n}_{1}}{\mathrm{n}_{0}+\mathrm{n}_{1}} \\
& =\frac{\mathrm{e}}{\mathrm{m}} \nabla \phi-\frac{1}{2} \nabla\left(2 \frac{\mathrm{e}^{2}}{\mathrm{~m}^{2} \mathrm{c}^{2}}\left(\mathbf{A}_{\mathrm{L}} \cdot \mathbf{A}_{1}\right)\right)-3 \mathrm{v}_{\mathrm{th}}{ }^{2} \frac{\nabla \mathrm{n}_{1}}{\mathrm{n}_{0}}
\end{aligned}
$$

for the momentum equation, and

$$
\frac{\partial \mathrm{n}_{1}}{\partial \mathrm{t}}+\mathrm{n}_{0}(\nabla \cdot \mathrm{u})=0
$$

for the continuity equation. These equations can be combined by taking the partial derivative with respect to $t$ of (2.46) and the divergence of (2.45). I.e.,

$$
\frac{\partial^{2} \mathrm{n}_{1}}{\partial \mathrm{t}^{2}}+\mathrm{n}_{0} \frac{\partial}{\partial \mathrm{t}}(\nabla \cdot \mathrm{u})=0
$$

and

$$
\nabla \cdot \frac{\partial \mathrm{u}}{\partial \mathrm{t}}=\frac{\mathrm{e}}{\mathrm{m}} \nabla^{2} \phi-\frac{\mathrm{e}^{2}}{\mathrm{~m}^{2} \mathrm{c}^{2}} \nabla^{2}\left(\mathbf{A}_{\mathrm{L}} \cdot \mathbf{A}_{1}\right)-3 \mathrm{v}_{\mathrm{th}}{ }^{2} \frac{\nabla^{2} \mathrm{n}_{1}}{\mathrm{n}_{\mathbf{0}}}
$$

Substitution of equation (2.47) into equation (2.48), recalling $\nabla^{2} \phi=4 \pi \mathrm{en}_{1}$ (equation (2.27)), and keeping only the first order fluctuation term yields

$$
\frac{\partial^{2} \mathrm{n}_{1}}{\partial \mathrm{t}^{2}}-3 \mathrm{v}_{\mathrm{th}}^{2} \nabla^{2} \mathrm{n}_{1}=\frac{\mathrm{e}^{2} \mathrm{n}_{0}}{\mathrm{~m}^{2} \mathrm{c}^{2}} \nabla^{2}\left(\mathbf{A}_{\mathrm{L}} \cdot \mathbf{A}_{1}\right)-\omega_{\mathrm{pe}}^{2} \mathrm{n}_{1}
$$

which can be rewritten as

$$
\left(\frac{\partial^{2}}{\partial t^{2}}-3 v_{t h}^{2} \nabla^{2}+\omega_{p e}^{2}\right) n_{1}=\frac{e^{2} n_{0}}{m^{2} c^{2}} \nabla^{2}\left(\mathbf{A}_{L} \cdot \mathbf{A}_{1}\right)
$$

Equation (2.50) describes the amplitude of the electron oscillation and is the third coupled equation. $\mathbf{A}_{\mathrm{L}} \cdot \mathbf{A}_{1}$ is the spatially periodic ponderomotive force driving term. The normal mode disperion relation for the electron plasma wave (equation (2.5)) can be obtained from equation (2.50) by letting the right hand side equal zero (i.e., no coupling) and Fourier analyzing. This gives

$$
\left(-\omega_{\mathrm{e}}^{2}+3 \mathrm{v}_{\mathrm{th}}{ }^{2} \mathrm{k}^{2}+\omega_{\mathrm{pe}}^{2}\right) \mathrm{n}_{1}=0
$$


which becomes

$$
\omega_{\mathrm{e}}^{2}=\omega_{\mathrm{pe}}^{2}+3 \mathrm{k}^{2} \mathrm{v}_{\mathrm{th}}^{2}
$$

since $\mathrm{n}_{1} \neq 0$. This is equation (2.5).

To solve the coupled differential equations ((2.39) and (2.50)), the waves are defined as the product of a fast oscillation and a slowly varying envelope and the problem is limited to one spatial dimension. (A 1-D analysis is adequate for a plane wave incident upon a homogeneous plasma.) This provides a pair of equations for the envelopes of the scattered light wave and the electron oscillation amplitude which can then be solved. Defining the waves as a fast oscillation times a slowly varying envelope gives

$$
\begin{aligned}
A_{1} & =a_{s}(x, t) e^{i\left(k_{s} x-\omega_{s} t\right)}+\text { complex conjugate } \\
A_{L} & =a_{0}(x, t) e^{i\left(k_{0} x-\omega_{0} t\right)}+\text { complex conjugate } \\
n_{1} & =n(x, t) e^{i\left(k_{e} x-\omega_{e} t\right)}+\text { complex conjugate }
\end{aligned}
$$

These expressions are valid for both forward scatter and backscatter. For forward scatter, $k_{s}=\left|k_{s}\right|$ while for backscatter, $k_{s}=-\left|k_{s}\right|$. Substituting these expressions into equations (2.39) and (2.50), assuming the scattered light wave, $a_{s}(x, t)$, is slowly varying in both space and time, and the electron oscillation amplitude, $\mathrm{n}(\mathrm{x}, \mathrm{t})$, is slowly varying in space only, i.e.,

$$
\begin{aligned}
\frac{\partial^{2} \mathrm{a}_{\mathrm{s}}(\mathrm{x}, \mathrm{t})}{\partial \mathrm{x}^{2}} & <i \mathrm{ik}_{\mathrm{s}} \frac{\partial a_{s}(x, t)}{\partial \mathrm{x}} \\
\frac{\partial \mathrm{a}_{\mathrm{s}}(\mathrm{x}, \mathrm{t})}{\partial \mathrm{x}} & <\mathrm{ik}_{\mathrm{s}} a_{s}(x, t) \\
\frac{\partial^{2} \mathrm{a}_{\mathrm{s}}(\mathrm{x}, \mathrm{t})}{\partial \mathrm{t}^{2}}< & <\mathrm{i} \omega_{s} \frac{\partial \mathrm{a}_{\mathrm{s}}(\mathrm{x}, \mathrm{t})}{\partial \mathrm{t}} \\
\frac{\partial \mathrm{a}_{\mathrm{s}}(\mathrm{x}, \mathrm{t})}{\partial \mathrm{t}} & <i \omega_{\mathrm{s}} \mathrm{a}_{\mathrm{s}}(\mathrm{x}, \mathrm{t}) \\
\frac{\partial^{2} \mathrm{n}(\mathrm{x}, \mathrm{t})}{\partial \mathrm{x}} & <i \mathrm{ik}_{\mathrm{s}} \frac{\partial \mathrm{n}(\mathrm{x}, \mathrm{t})}{\partial \mathrm{x}} \\
\frac{\partial \mathrm{n}(\mathrm{x}, \mathrm{t})}{\partial \mathrm{x}} & <i \mathrm{ik}_{\mathrm{s}} \mathrm{n}(\mathrm{x}, \mathrm{t})
\end{aligned}
$$


The amplitude of the electron plasma wave envelope has not been assumed to be slowly varying in time because that assumption implies that

$$
\frac{\partial \mathrm{n}(\mathrm{x}, \mathrm{t})}{\partial \mathrm{t}} \ll \omega_{\mathrm{e}} \mathrm{n}(\mathrm{x}, \mathrm{t})
$$

For short pulse intense lasers, this assumption is not necessarily valid since the growth rate, $\gamma$, which is given by $\frac{1}{n} \frac{\partial \mathrm{n}}{\partial \mathrm{t}}$, can be on the order of or greater than $\omega_{\mathrm{e}}$ (i.e., strongly coupled). For the scattered light wave, $\omega_{\mathrm{s}} \gg \omega_{\mathrm{e}}$, so $\omega_{\mathrm{s}} \gg \gamma$ for all the parameters of interest in this work, allowing application of the slowly varying approximation for $\mathrm{a}_{\mathrm{s}}$. Use of the slowly varying approximation gives for the scattered light wave envelope

$$
\begin{aligned}
& {\left[2 \mathrm{ik}_{\mathrm{s}} \mathrm{c}^{2} \frac{\partial \mathrm{a}_{\mathrm{s}}}{\partial \mathrm{x}}+2 \mathrm{i} \omega_{s} \frac{\partial \mathrm{a}_{\mathrm{s}}}{\partial \mathrm{t}}-\left(\mathrm{k}_{\mathrm{s}}{ }^{2} \mathrm{c}^{2}-\omega_{s}{ }^{2}+\omega_{\mathrm{pe}}{ }^{2}\right) \mathrm{a}_{\mathrm{s}}\right] \mathrm{e}^{\mathrm{i}\left(\mathrm{k}_{\mathrm{s}} \mathrm{x}-\omega_{\mathrm{s}} \mathrm{t}\right)}+\text { comp. conj. }} \\
& \quad=\frac{4 \pi \mathrm{e}^{2}}{\mathrm{~m}}\left\{\mathrm{na} \mathrm{a}_{\mathrm{L}} \mathrm{e}^{\mathrm{i}\left(\left(\mathrm{k}_{0}+\mathrm{k}_{\mathrm{e}}\right) \mathrm{x}-\left(\omega_{0}+\omega_{e}\right) \mathrm{t}\right)}+\mathrm{na}_{\mathrm{L}} \mathrm{e}^{\mathrm{i}\left(\left(\mathrm{k}_{\mathrm{e}}-\mathrm{k}_{0}\right) \mathrm{x}-\left(\omega_{\mathrm{e}}-\omega_{0}\right) \mathrm{t}\right)}\right. \\
& \left.\quad+\mathrm{n}^{*} \mathrm{a}_{\mathrm{L}} \mathrm{e}^{-\mathrm{i}\left(\left(\mathrm{k}_{\mathrm{e}}-\mathrm{k}_{\mathrm{o}}\right) \mathrm{x}-\left(\omega_{\mathrm{e}}-\omega_{0}\right) \mathrm{t}\right)}+\mathrm{n}^{*} \mathrm{a}_{\mathrm{L}}{ }^{*} \mathrm{e}^{-\mathrm{i}\left(\left(\mathrm{k}_{0}+\mathrm{k}_{\mathrm{e}}\right) \mathrm{x}-\left(\omega_{0}+\omega_{\mathrm{e}}\right) \mathrm{t}\right)}\right\}
\end{aligned}
$$

The electron oscillation envelope is given by

$$
\begin{aligned}
& {\left[\frac{\partial^{2} \mathrm{n}}{\partial \mathrm{t}^{2}}-2 \mathrm{i} \omega_{\mathrm{e}} \frac{\partial \mathrm{n}}{\partial \mathrm{t}}-6 \mathrm{iv}_{\mathrm{th}}{ }^{2} \mathrm{k}_{\mathrm{e}} \frac{\partial \mathrm{n}}{\partial \mathrm{x}}\right] \mathrm{e}^{\mathrm{i}\left(\mathrm{k}_{\mathrm{e}} \mathrm{x}-\omega_{\mathrm{e}} \mathrm{t}\right)}+\text { comp. conj. }} \\
& =-\frac{\mathrm{e}^{2} \mathrm{n}_{0}}{\mathrm{~m}^{2} \mathrm{c}^{2}}\left(\mathrm{k}_{0}-\mathrm{k}_{\mathrm{s}}\right)^{2}\left[\mathrm{a}_{\mathrm{L}} \mathrm{a}_{\mathrm{s}}{ }^{*} \mathrm{e}^{\mathrm{i}\left(\left(\mathrm{k}_{0}-\mathrm{k}_{s}\right) \mathrm{x}-\left(\omega_{0}-\omega_{s}\right) t\right)}+\mathrm{a}_{\mathrm{L}}{ }^{*} \mathrm{a}_{\mathrm{s}} \mathrm{e}^{-\mathrm{i}\left(\left(\mathrm{k}_{0}-\mathrm{k}_{\mathrm{s}}\right) \mathrm{x}-\left(\omega_{0}-\omega_{s}\right) \mathrm{t}\right)}\right. \\
& \left.+a_{L} a_{s} e^{i\left(\left(k_{0}+k_{s}\right) x-\left(\omega_{0}+\omega_{s}\right) t\right)}+a_{L}{ }^{*} a_{s}{ }^{*} e^{-i\left(\left(k_{0}+k_{s}\right) x-\left(\omega_{0}+\omega_{s}\right) t\right)}\right] .
\end{aligned}
$$

The terms $\frac{\partial^{2}}{\partial x^{2}}\left(a_{L} a_{s}^{*}\right)$ and $i\left(k_{0}-k_{s}\right) \frac{\partial}{\partial x}\left(a_{L} a_{s}^{*}\right)$ on the right hand side of equation (2.53) have been neglected based on the slowly varying approximations shown above.

Using the frequency and wavenumber matching conditions for the Stokes wave, "+" of equations (2.1) and (2.2), only two terms on the right hand side of equation(2.52) contribute to the amplitude of the scattered wave envelope and only two terms on the right hand side of equation (2.53) contribute to the electron oscillation amplitude. The other terms on the right hand side in each equation are associated with the antiStokes wave and will not be resonant for stimulated Raman backscatter. I.e., they will not have an appreciable contribution to the amplitude. For forward SRS, these terms can be resonant and should not be neglected. Equation (2.52), when only the 
Stokes wave is resonant, reduces to two complex conjugate equations one of which is given by

$$
\frac{\partial \mathrm{a}_{\mathrm{s}}}{\partial \mathrm{x}}+\frac{\omega_{\mathrm{s}}}{\mathrm{k}_{\mathrm{s}} \mathrm{c}^{2}} \frac{\partial \mathrm{a}_{\mathrm{s}}}{\partial \mathrm{t}}=-\frac{2 \mathrm{i} \pi \mathrm{e}^{2}}{\mathrm{k}_{\mathrm{s}} \mathrm{mc}^{2}} \mathrm{n}^{*} \mathrm{a}_{\mathrm{L}}
$$

where equation (2.4) was used. Equation (2.53) similarly reduces to

$$
\frac{\mathrm{i}}{2 \omega_{\mathrm{e}}} \frac{\partial^{2} \mathrm{n}^{*}}{\partial \mathrm{t}^{2}}+\frac{\partial \mathrm{n}^{*}}{\partial \mathrm{t}}+3 \mathrm{v}_{\mathrm{th}}{ }^{2} \frac{\mathrm{k}_{\mathrm{e}}}{\omega_{\mathrm{e}}} \frac{\partial \mathrm{n}^{*}}{\partial \mathrm{x}}=\frac{\mathrm{i} \omega_{\mathrm{pe}}}{8 \pi \mathrm{mc}^{2}}\left(\mathrm{k}_{0}-\mathrm{k}_{\mathrm{s}}\right)^{2} \mathrm{a}_{\mathrm{L}}{ }^{*} \mathrm{a}_{\mathrm{s}}
$$

and a conjugate equation. For forward SRS, in contrast to backscattered SRS, the anti-Stokes mode is also resonant and the right hand side of equation (2.54) becomes $\left(\frac{-2 i \pi e^{2}}{k_{s} m c^{2}}\right)\left(n^{*} a_{L}+n a_{L}\right)$ while for equation (2.55) the term on the right hand side becomes $\left(\frac{i \omega_{p e}}{8 \pi m c^{2}}\right)\left(k_{0}-k_{s}\right)^{2}\left(a_{L}{ }^{*} a_{s}+a_{L}{ }^{*} a_{s}{ }^{*}\right)$.

The equations, (2.54) and (2.55), represent the coupled differential equations for the envelopes of the scattered light wave amplitude and the electron oscillation amplitude. These two equations, when solved together, give a growing solution while the complex conjugate equations give a decaying solution. Equations (2.54) and (2.55) are the coupled envelope equations which describe the growth of the scattered light and electron plasma wave envelopes when pump depletion has been neglected. These equations are valid only for backscatter $\left(k_{s}=-\left|k_{s}\right|\right)$ since only the Stokes mode is retained. To describe the forward scatter problem $\left(k_{s}=\left|k_{s}\right|\right)$, the anti-Stokes mode is also resonant and the coupled equations are modified, as described above. These coupled equations illustrate mathematically the feedback loop shown in figure 2.1(b). The amplitude of the scattered light wave is driven by a coupling between the density fluctuation and the incident light wave. Coupling between the incident light wave and the scattered light wave serves as the driving term for the density fluctuation (plasma wave).

The driving term for the growth of the plasma wave is proportional to $\left(k_{0}-k_{s}\right)^{2}$, as seen in equation (2.55). This implies that the maximum growth occurs when the wavenumber of the scattered light wave, $k_{s}$, is of opposite sign to the wavenumber of the incident light wave, $k_{0}$. In other words, direct backscatter (where $k_{s}=-\left|k_{s}\right|$ ) will grow larger than forward scatter (where $k_{s}=\left|k_{s}\right|$ ). 
To illustrate the behavior of the solutions to the coupled equations, a finite difference code was written to numerically solve normalized versions of equations (2.54) and (2.55) for stimulated Raman backscatter. These same equations can be used to solve for the growth of forward SRS, but since the anti-Stokes mode was neglected as nonresonant, the remaining discussion will be confined strictly to stimulated Raman backscatter. A grid of 500 spatial points $(\mathrm{x})$ and 1250 temporal points $(\mathrm{t})$ was used to solve the equations. The time steps ( $\leq 10 \mathrm{fsec}$ ) were determined by the total propagation time of the laser pulse through the plasma. The spatial steps were given by $\mathrm{v}_{\mathrm{g}} \Delta \mathrm{t}$, where $\mathrm{v}_{\mathrm{g}}$ is the group velocity of the scattered light in the plasma.

$$
v_{\mathrm{g}}=c \sqrt{1-\frac{\omega_{\mathrm{pe}^{2}}}{\omega_{\mathrm{s}}^{2}}}
$$

Numerical stability is assured since the Courant condition, given by $\mathrm{v}_{\mathrm{g}} \mathrm{dt} / \mathrm{dx} \leq 1$, is satisfied.[94] In this code, $\mathrm{v}_{\mathrm{g}} \mathrm{dt} / \mathrm{dx}$ was fixed at 0.1 . The incident laser field used was a temporal gaussian,

$$
a_{L}(x, t)=a_{0} e^{-\left(4 \ln 2\left(t-x / v_{g 1}\right) / 2 \tau^{2}\right)}
$$

where $\tau=600 \mathrm{fsec}$, the temporal full width at half maximum of the intensity, and $\mathrm{v}_{\mathrm{g} 1}$ is the group velocity of the incident laser light in the plasma.

$$
\mathrm{v}_{\mathrm{g} 1}=\mathrm{c} \sqrt{1-\frac{\omega_{\mathrm{pe}}^{2}}{\omega_{0}^{2}}}
$$

Equations (2.54) and (2.55) were also solved neglecting the $\frac{\partial^{2} \mathrm{n}^{*}}{\partial t^{2}}$ term, i.e.,

$$
\begin{aligned}
\frac{\partial \mathrm{a}_{\mathrm{s}}}{\partial \mathrm{x}}+\frac{\omega_{\mathrm{s}}}{\mathrm{k}_{\mathrm{s}} \mathrm{c}^{2}} \frac{\partial \mathrm{a}_{\mathrm{s}}}{\partial \mathrm{t}} & =-\frac{2 \mathrm{i} \pi \mathrm{e}^{2}}{\mathrm{k}_{\mathrm{s}} \mathrm{mc}^{2}{ }^{*} \mathrm{a}_{\mathrm{L}}} \\
\frac{\partial \mathrm{n}^{*}}{\partial \mathrm{t}}+3 \mathrm{v}_{\mathrm{e}}{ }^{2} \frac{\mathrm{k}_{\mathrm{e}}}{\omega_{\mathrm{e}}} \frac{\partial \mathrm{n}^{*}}{\partial \mathrm{x}} & =\frac{\mathrm{i} \omega_{\mathrm{pe}}}{8 \pi \mathrm{mc}^{2}}\left(\mathrm{k}_{0}-\mathrm{k}_{\mathrm{s}}\right)^{2} \mathrm{a}_{\mathrm{L}}{ }^{*} \mathrm{a}_{\mathrm{s}}
\end{aligned}
$$

in order to determine the contribution of this second order term for the short pulse problem. This term is a measure of the coupling strength and becomes important when the growth rate of the instability is on the order of the plasma wave frequency, $\omega_{e}$, as discussed earlier. In the remainder of this section, $\frac{\partial^{2} \mathrm{n}^{*}}{\partial t^{2}}$ will be referred to as $\Gamma_{\text {cs }}$. 
The numerical solution for parameters typical of the backscatter experiments discussed later in this work are shown in figure 2.3. For this calculation, the electron density was fixed at $7 \times 10^{17} \mathrm{~cm}^{-3}$, the peak laser intensity was $1 \times 10^{17} \mathrm{~W} / \mathrm{cm}^{2}$, and the propagation time was $4000 \mathrm{fsec}$ (6.67 times the laser pulse width). The laser pulse was a transform-limited $600 \mathrm{fsec}$ pulse at a wavelength of $1.0527 \mu \mathrm{m}$. The SRS was assumed to grow from a white noise source given by $10^{-10}$ of the background electron density (uniform). Figure 2.3(a) shows the inital and final positions of the
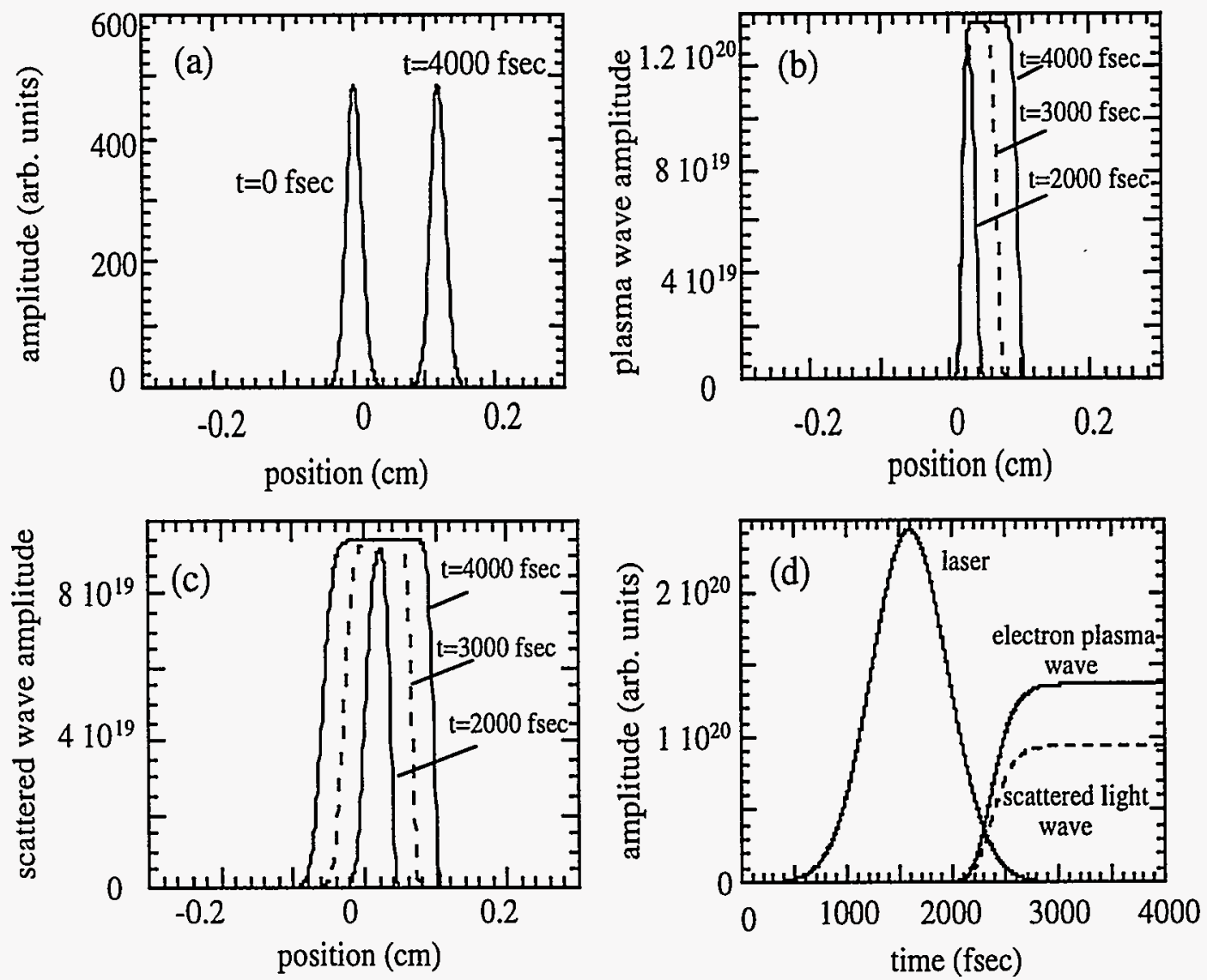

Figure 2.3: Results of the backscatter envelope approximation. (a) Initial and final positions of the propagating laser pulse, (b) evolution of the growing electron plasma wave, (c) evolution of the scattered light wave, and (d) temporal evolution of the laser, the electron plasma wave, and the scattered light wave. These curves were obtained for $\mathrm{I}=1 \times 10^{17} \mathrm{~W} / \mathrm{cm}^{2}$ and $\mathrm{n}_{0}=7 \times 10^{17} \mathrm{~cm}^{-3}$.

laser pulse. In this calculation, the laser pulse begins centered at $\mathrm{x}=0$ and propagates 
to the right (forward direction). As the laser propagates through the plasma, the amplitude of the electron plasma wave grows, as illustrated in figure 2.3(b). The plasma wave extends in the direction of laser propagation as it grows, indicating that this wave is propagating in the same direction as the laser, but with $\mathrm{v}_{\mathrm{g}} \ll \mathrm{c}$. Growth of the scattered light wave as the laser propagates is shown in figure 2.3(c). The scattered light wave envelope extends to the left of the initial laser position as it grows (backscatter). Figure 2.3(d) depicts the growth of the electron plasma wave and the scattered light wave (Stokes mode) as a function of time at a particular location in the plasma $(x=0.048 \mathrm{~cm})$. Early in time, when the laser intensity is low, there is little growth of the plasma and scattered light waves. As the high intensity portion of the laser moves past, the waves experience large growth. Later in time, after the laser has passed, there is no longer a driving term and the waves no longer grow (i.e., $t \geq 3000$ fsec). The final amplitude of the plasma wave is $1.93 \times 10^{22}$ times higher than the initial amplitude, indicating that, for this case, the plasma wave grew numerically by 51 e-foldings (in the absence of damping and nonlinear effects, both of which should occur in the experiments).

The growth of the plasma wave is a function of the incident intensity of the short pulse laser, as shown in figure 2.4(a). In this plot, the factor by which the plasma wave amplitude grew over the inital noise level is plotted as a function of intensity for a fixed electron density of $1 \times 10^{17} \mathrm{~cm}^{-3}$ and a propagation time of $4000 \mathrm{fsec}$. Results obtained solving the coupled partial differential equations, with (solid line) ((2.54) and (2.55)) and without (dashed line) ((2.59) and (2.60)) $\Gamma_{\mathrm{cs}}$ are also shown in figure 2.4. The solid circles show the growth obtained using the analytic expression for the growth rate (equation (2.68), derived later in this chapter). There was good agreement between the calculation result and the analytic result. It is clear that at the higher intensities, inclusion of $\Gamma_{\mathrm{cs}}$, the measure of the coupling strength, changes the total growth. The level of growth increases with intensity, as expected.

Plasma wave growth as a function of electron density is plotted in figure 2.4(b) for a fixed intensity of $7 \times 10^{16} \mathrm{~W} / \mathrm{cm}^{2}$ and a 4000 fsec propagation time. The solid line 

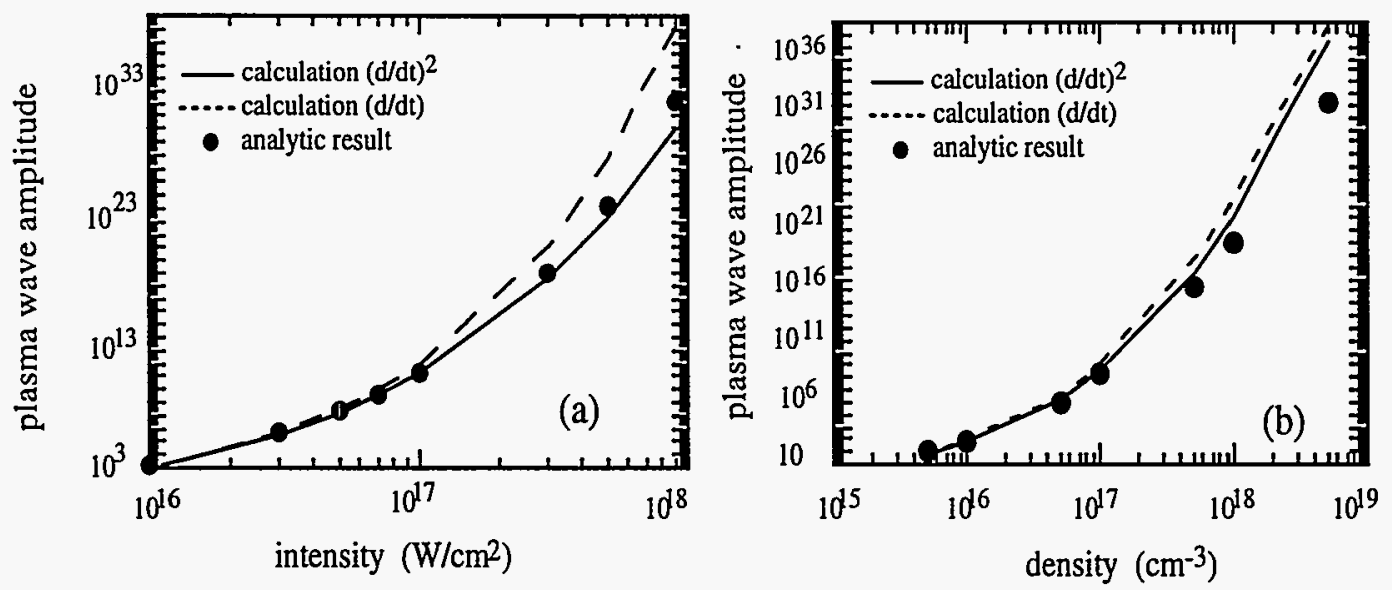

Figure 2.4: Growth of the electron plasma wave as a function of (a) peak laser intensity $\left(\mathrm{n}_{0}=1 \times 10^{17} \mathrm{~cm}^{-3}\right)$ and $(b)$ plasma density $\left(\mathrm{I}=7 \times 10^{16} \mathrm{~W} / \mathrm{cm}^{2}\right)$. The propagation time was $4000 \mathrm{fsec}$.

represents the growth obtained keeping the $\Gamma_{c s}$ term in the coupled equations and the dashed line shows the result obtained without this second derivative. The solid circles represent the growth obtained analytically using equation (2.68). Again, reasonable agreement is observed. The calculated results obtained with and without $\Gamma_{\text {cs }}$ were very similar for all densities. The growth of the electron plasma wave increased with increasing density, as expected.

In figure 2.3 , the waves grew from a uniform noise source. Since there is no guarantee that the noise level from which SRS grows is completely uniform [102], the case of a random spatial noise source was considered. If the initial density fluctuation is nonuniform, it is possible that the electron plasma and scattered light waves produced may no longer be smooth since they are amplifications of the noise. Figure 2.5 shows the shape of the electron plasma (b) and scattered light (c) waves obtained for a random initial density perturbation.(a). In this calculation, the plasma density was fixed at $7 \times 10^{17} \mathrm{~cm}^{-3}$, the peak laser intensity was $1 \times 10^{17} \mathrm{~W} / \mathrm{cm}^{2}$, and the propagation time was $4000 \mathrm{fsec}$. The random noise source used in the calculation was generated with a random number generator. The fluctuations varied from $5 \times 10^{-11}$ 

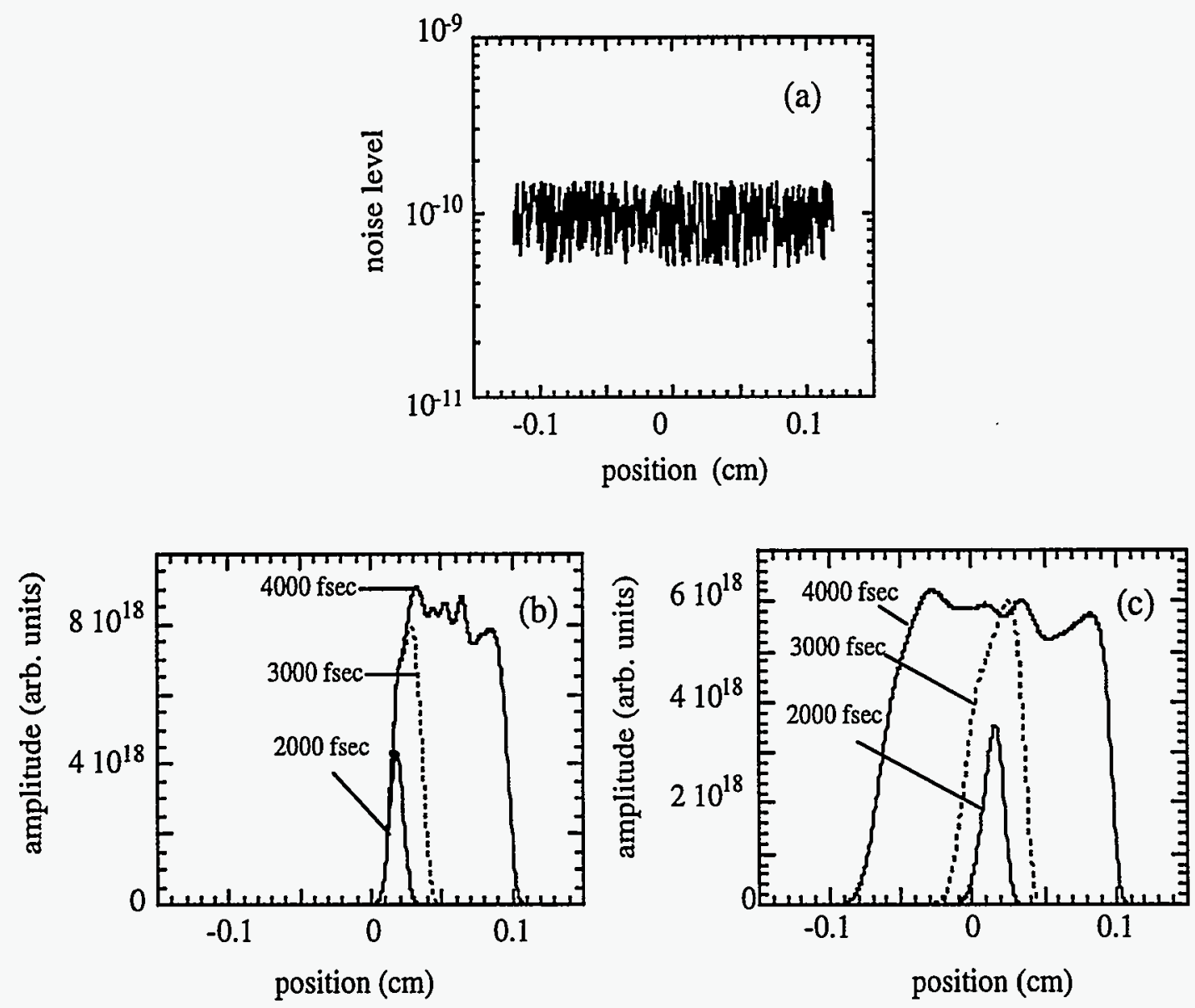

Figure 2.5: Solution to coupled equations for a random initial density perturbation. (a) The random perturbation, (b) evolution of the electron plasma wave, and (c) evolution of the scattered light wave. In this calculation, $\mathrm{n}_{0}=7 \times 10^{17} \mathrm{~cm}^{-3}, \mathrm{I}=$ $1 \times 10^{17} \mathrm{~W} / \mathrm{cm}^{2}$. The laser propagated for $4000 \mathrm{fsec}$. 
to $1.5 \times 10^{-10}\left(10^{-10} \pm 5 \times 10^{-10}\right)$. In this calculation, both the electron plasma and scattered light waves developed modulated behavior. While the degree of modulation on these waves was much less severe than the initial noise fluctuation, the presence of modulations indicates that the initial spatial noise level from which SRS grows can effect the electron plasma and scattered light waves generated in the SRS process.

\subsection{The Dispersion Relation}

Solving the coupled equations can provide a variety of information about the behavior and evolution of the waves associated with stimulated Raman scattering. The coupled equations can provide information about the growth of SRS in the transient regime (i.e., growth of the instability in less than one laser pulse length) and in the steady state regime (i.e., growth over several pulse lengths). Solving the dispersion relation, which is derived from these coupled equations, is simpler, however, since it reduces to a polynomial. Unlike the coupled equations, the dispersion relation only produces accurate results in the steady state regime. This polynomial, when solved for its roots, can be used to predict where (in $\omega$-space) and in what direction (k-space) SRS will grow. The dispersion relation, like the coupled equations, can be used to model both the forward scatter and backscatter SRS problems. The growth rate of SRS can also be obtained from the dispersion relation. In this section the nonrelativistic dispersion relation and growth rates for forward and back-scattered SRS are derived and solved, following the formalism of Forslund and Kindel. [51] The relativistic dispersion relation is also solved and the results compared with the nonrelativistic solution. The results shown in this section were obtained with a root solving routine in IDL. 


\subsubsection{Nonrelativisitic}

The nonrelativistic dispersion relation is derived from the coupled differential equations, (2.39) and (2.50). First, the large amplitude light wave, $\mathbf{A}_{\mathrm{L}}$, is written as

$$
\mathbf{A}_{\mathrm{L}}=\mathrm{a}_{0} \cos \left(\mathrm{k}_{0} \cdot \mathrm{x}-\omega_{0} \mathrm{t}\right)
$$

and substituted into equations (2.39) and (2.50). The equations are then Fourieranalyzed. Fourier analysis implies the substitutions:

$$
\begin{gathered}
\frac{\partial}{\partial \mathrm{t}} \rightarrow \mathrm{i}\left(\omega+\mathrm{m} \omega_{0}\right) \\
\nabla \rightarrow-\mathrm{i}\left(\mathrm{k}+\mathrm{mk}_{0}\right)
\end{gathered}
$$

where $m=0, \pm 1$. The coupled equations can now be written as

$$
\begin{aligned}
& \left(\omega^{2}-c^{2} \mathrm{k}^{2}-\omega_{\mathrm{pe}}{ }^{2}\right) \mathbf{A}_{1}(\mathrm{k}, \omega)= \\
& \quad \frac{1}{2} \frac{\omega_{\mathrm{pe}}{ }^{2}}{\mathrm{n}_{0}} \mathbf{A}_{0}\left[\mathrm{n}_{1}\left(\mathrm{k}-\mathrm{k}_{0}, \omega-\omega_{0}\right)+\mathrm{n}_{1}\left(\mathrm{k}+\mathrm{k}_{0}, \omega+\omega_{0}\right)\right] \\
& \left(\omega^{2}-\omega_{\mathrm{e}}{ }^{2}\right) \mathrm{n}_{1}(\mathrm{k}, \omega)= \\
& \frac{\mathrm{e}^{2} \mathrm{n}_{0}}{\mathrm{~m}^{2} \mathrm{c}^{2}} \mathrm{k}^{2} \mathbf{A}_{0} \cdot\left[\mathrm{A}_{1}\left(\mathrm{k}-\mathrm{k}_{0}, \omega-\omega_{0}\right)+\mathbf{A}_{1}\left(\mathrm{k}+\mathrm{k}_{0}, \omega+\omega_{0}\right)\right]
\end{aligned}
$$

where $\omega_{\mathrm{e}}$ is given by equation (2.5). Using equation (2.62) to eliminate $\mathbf{A}_{1}$ from equation (2.63) and neglecting $n_{1}\left(k-2 k_{0}, \omega-2 \omega_{0}\right)$ and $n_{1}\left(k+2 k_{0}, \omega+2 \omega_{0}\right)$ (very nonresonant terms), yields

$$
\begin{aligned}
\left(\omega^{2}-\omega_{\mathrm{e}}^{2}\right) \mathrm{n}_{1}(\mathrm{k}, \omega)= & \mathrm{k}^{2} \frac{\mathrm{e}^{2} \mathrm{n}_{0}}{\mathrm{~m}^{2} \mathrm{c}^{2}}\left(\frac{1}{2} \frac{\omega_{\mathrm{pe}}{ }^{2}}{\mathrm{n}_{0}}\right) \mathbf{A}_{0} \cdot\left[\mathrm{A}_{1} \frac{\mathrm{n}_{1}(\mathrm{k}, \omega)}{\left(\omega-\omega_{0}\right)^{2}-\mathrm{c}^{2}\left(\mathrm{k}-\mathrm{k}_{0}\right)^{2}-\omega_{\mathrm{pe}}{ }^{2}}\right. \\
& \left.+\mathrm{A}_{1} \frac{\mathrm{n}_{1}(\mathrm{k}, \omega)}{\left(\omega+\omega_{0}\right)^{2}+\mathrm{c}^{2}\left(\mathrm{k}+\mathrm{k}_{0}\right)^{2}-\omega_{\mathrm{pe}}{ }^{2}}\right]
\end{aligned}
$$

which can be reduced to

$$
\omega^{2}-\omega_{\mathrm{e}}^{2}=\frac{1}{4} \mathrm{k}^{2} \mathrm{v}_{\mathrm{osc}}{ }^{2} \omega_{\mathrm{pe}}^{2}\left[\frac{1}{\mathrm{D}\left(\omega-\omega_{0}, \mathrm{k}-\mathrm{k}_{0}\right)}+\frac{1}{\mathrm{D}\left(\omega+\omega_{0}, \mathrm{k}+\mathrm{k}_{0}\right)}\right]
$$

where $\mathrm{D}(\omega, \mathrm{k})=\omega^{2}-\mathrm{c}^{2} \mathrm{k}^{2}-\omega_{\mathrm{pe}}{ }^{2}$. This equation is the nonrelativistic dispersion relation for stimulated Raman scattering. It illustrates the relationship between the 
frequency and wavenumber of the plasma wave that must be satisfied in order for the wave to grow. $\mathrm{D}\left(\omega-\omega_{0}, \mathrm{k}-\mathrm{k}_{0}\right)$ is commonly referred to as the Stokes term and $\mathrm{D}\left(\omega+\omega_{0}, \mathrm{k}+\mathrm{k}_{0}\right)$ is the anti-Stokes term. Only the first Stokes and anti-Stokes features have been retained in this result. Higher order Stokes and anti-Stokes modes, neglected as very nonresonant in this derivation, can become resonant for forward Raman scatter driven with an intense laser pulse, however, as observed in chapter 4.

\section{Backscatter}

To examine the problem of stimulated Raman backscatter, equation (2.65) is solved for $\mathrm{k} \approx 2 \mathrm{k}_{0}$, the wavenumber for backscatter as determined by the matching condition (2.2) (see section 2.1). Since the wavenumber is large, the anti-Stokes term on the right hand side of equation (2.65), $\left(\left(\omega+\omega_{0}\right)^{2}-c^{2}\left(k+k_{0}\right)^{2}-\omega_{p e}\right)^{-1}$, is very nonresonant and can be neglected. This allows equation (2.65) to be reduced to

$$
\omega^{2}-\omega_{\mathrm{e}}^{2}=\frac{1}{4} \mathrm{k}^{2} \mathrm{v}_{\mathrm{osc}}{ }^{2} \omega_{\mathrm{pe}}{ }^{2}\left[\frac{1}{\left(\omega-\omega_{0}\right)^{2}-\mathrm{c}^{2}\left(\mathrm{k}-\mathrm{k}_{0}\right)^{2}-\omega_{\mathrm{pe}}{ }^{2}}\right] .
$$

The growth rate for backscattered SRS can be obtained from this equation. Let $\omega=\omega_{\mathrm{e}}+\delta \omega$, where $\delta \omega \ll \omega_{\mathrm{e}}$. Equation (2.66) can then be rewritten to give

$$
2 \omega_{\mathrm{e}} \delta \omega\left[\left(\omega_{\mathrm{e}}-\omega_{0}\right)^{2}-\mathrm{c}^{2}\left(\mathrm{k}-\mathrm{k}_{0}\right)^{2}-\omega_{\mathrm{pe}}{ }^{2}+2 \delta \omega \omega_{\mathrm{e}}-2 \omega_{0} \delta \omega\right]=\frac{1}{4} \omega_{\mathrm{pe}}{ }^{2} \mathrm{k}^{2} \mathrm{v}_{\mathrm{osc}}{ }^{2}
$$

Since growth is largest when the scattered light wave is also resonant, i.e.,

$$
\left(\omega_{\mathrm{e}}-\omega_{0}\right)^{2}-\mathrm{c}^{2}\left(\mathrm{k}-\mathrm{k}_{0}\right)^{2}-\omega_{\mathrm{pe}}^{2}=0
$$

equation (2.67) reduces to

$$
(\delta \omega)^{2} \omega_{\mathrm{e}}\left(\omega_{\mathrm{e}}-\omega_{0}\right)=\frac{1}{16}{\omega_{\mathrm{pe}}}^{2} \mathrm{k}^{2} \mathrm{v}_{\mathrm{osc}}{ }^{2}
$$

Writing $\delta \omega=\mathrm{i} \gamma_{0}$ gives

$$
\gamma_{0}=\frac{\mathrm{kv}_{\mathrm{osc}}}{4}\left[\frac{\omega_{\mathrm{pe}}^{2}}{\omega_{\mathrm{e}}\left(\omega_{0}-\omega_{\mathrm{e}}\right)}\right]^{\frac{1}{2}}
$$


This is the growth rate for backscattered SRS in the weakly coupled regime. For certain intensities and densities, $\gamma_{0}>\omega_{\mathrm{pe}}$ and the instability is strongly coupled. ([40], [51]) The growth rate for strongly coupled SRS is given by

$$
\gamma_{\mathrm{sc}}=\frac{\sqrt{3}}{2}\left[\frac{\omega_{\mathrm{pe}}^{2} \mathrm{k}^{2} \mathrm{v}_{\mathrm{osc}}{ }^{2}}{\omega_{0}}\right]^{\frac{1}{3}}
$$

Note that the strongly coupled growth rate is proportional to $\mathrm{v}_{\text {osc }} \frac{2}{3}$ whereas $\gamma_{0} \propto \mathrm{v}_{\text {osc }}$.

To solve for the roots of equation (2.66), it is first rewritten as a polynomial,

$$
a_{4} \omega^{4}+a_{3} \omega^{3}+a_{2} \omega^{2}+a_{1} \omega+a_{0}=0
$$

where the coefficients are given by

$$
\begin{aligned}
& \mathrm{a}_{4}=1 \\
& \mathrm{a}_{3}=-2 \omega_{0} \\
& \mathrm{a}_{2}=2 \mathrm{c}^{2} \mathrm{kk}_{0}-\mathrm{c}^{2} \mathrm{k}^{2}-\omega_{\mathrm{e}}^{2} \\
& \mathrm{a}_{1}=2 \omega_{0} \omega_{\mathrm{e}}^{2} \\
& \mathrm{a}_{0}=\omega_{\mathrm{e}}^{2}\left(\mathrm{c}^{2} \mathrm{k}^{2}-2 \mathrm{c}^{2} \mathrm{kk_{0 }}\right)-\frac{1}{4} \omega_{\mathrm{pe}} \mathrm{k}^{2} \mathrm{v}_{\text {osc }}{ }^{2}
\end{aligned}
$$

This polynomial, when solved for the complex frequency $\omega$, produces two real roots and two complex conjugate roots. The complex conjugate roots describe the growth and decay of the electron plasma wave. The real part of the frequency, $\omega_{\mathrm{r}}$, gives information about where the electron plasma wave will grow (in frequency space) and the imaginary part, $\omega_{\mathrm{i}}$, is a measure of that growth. Figure 2.6(a) shows the roots of the polynomial for an undriven case, $\mathrm{n}_{0}=1 \times 10^{18} \mathrm{~cm}^{-3}$ and $\mathrm{v}_{\mathrm{osc}} / \mathrm{c}=0$, where the real part of the normalized frequency, $\omega_{\mathrm{r}} / \omega_{0}$ is plotted versus the normalized wavenumber, $k / k_{0}$. Curves $a$ and $b$ show the electron plasma waves and curve $c$ is the scattered light wave. The other scattered light wave is off this scale and doesn't interact with the other three waves. The same roots, found for $\mathrm{v}_{\mathrm{osc}} / \mathrm{c}=0.5$ and $\mathrm{n}_{0}=1 \times 10^{18} \mathrm{~cm}^{-3}$, are plotted in figure $2.6(\mathrm{~b})$. The effect of the driving term in the dispersion relation 

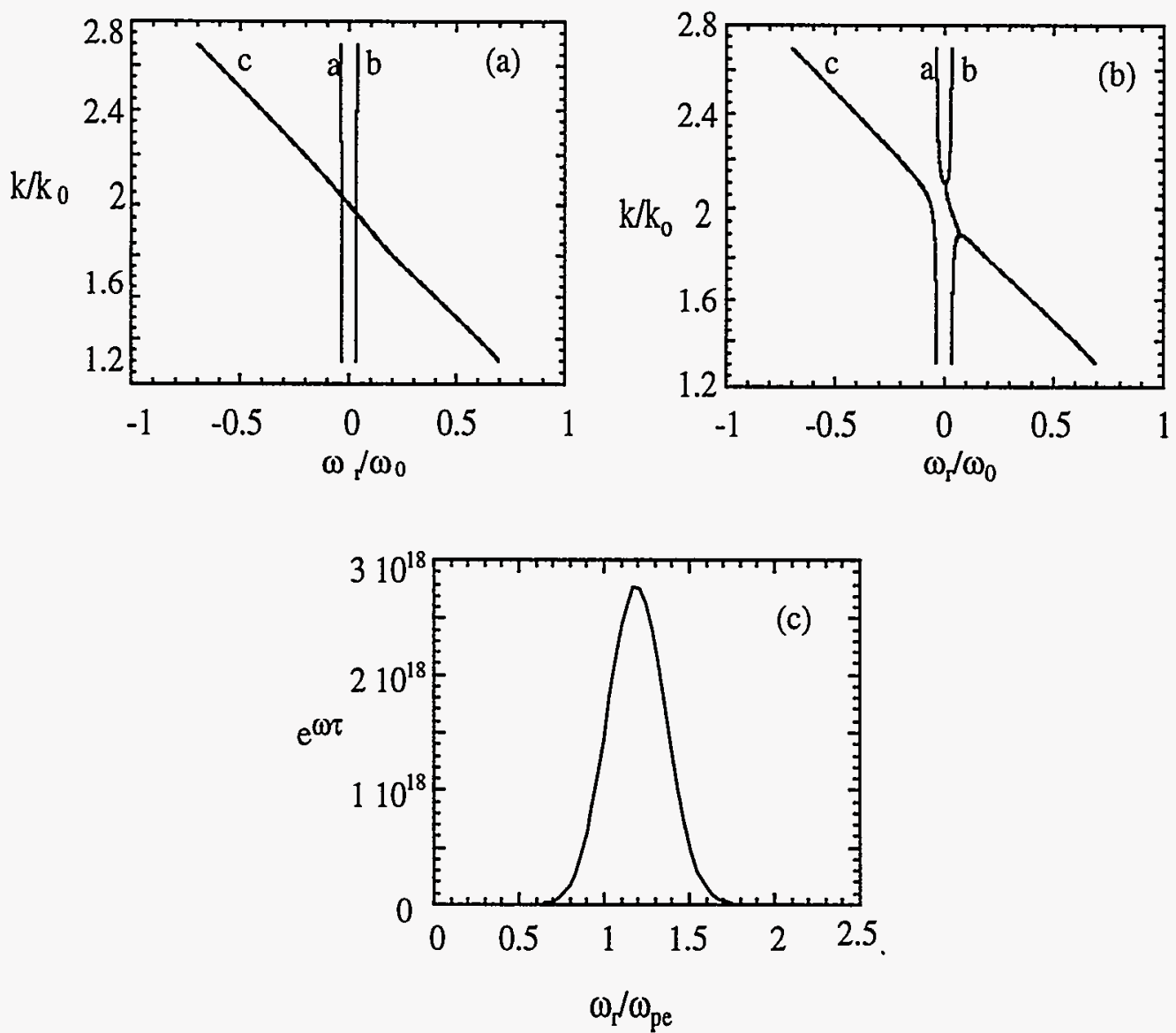

Figure 2.6: (a) Roots of the fourth order dispersion relation for an undriven system $\left(v_{\text {osc }} / \mathrm{c}=0, \mathrm{n}_{0}=1 \times 10^{18} \mathrm{~cm}^{-3}\right)$; (b) roots of the dispersion relation for a driven system $\left(\mathrm{v}_{\mathrm{osc}} / \mathrm{c}=0.5, \mathrm{n}_{0}=1 \times 10^{18} \mathrm{~cm}^{-3}\right)$ and $(\mathrm{c})$ the corresponding spectrum for the driven system.

is apparent in the coupling of the waves. Note that the coupling occurs for $\mathrm{k} / \mathrm{k}_{0} \approx 2$. Exponentiation of the imaginary part of the frequency, $\mathrm{e}^{\omega_{\mathrm{i}} \tau}$, where $r$ is the laser pulsewidth, plotted versus the real part of the normalized frequency, $\omega_{\mathrm{r}} / \omega_{\mathrm{pe}}=\omega_{\mathrm{e}} / \omega_{\mathrm{pe}}$, gives the power spectrum of the scattered light, as shown in figure 2.6(c). The SRS peak is located near $\omega_{\text {pe }}$, as expected from equation (2.1). The shape of the spectrum depends on both intensity $\left(\mathrm{v}_{\mathrm{osc}} / \mathrm{c}\right)$ and density, as illustrated in figure 2.7. In figure $2.7(\mathrm{a}), \omega_{\mathrm{i}} / \omega_{\mathrm{pe}}$ is plotted versus $\omega_{\mathrm{r}} / \omega_{\mathrm{pe}}$ for $\mathrm{v}_{\mathrm{osc}} / \mathrm{c}=0.8$ and $\mathrm{v}_{\mathrm{osc}} / \mathrm{c}=0.2$ for a fixed density of $\mathrm{n}_{0}=7 \times 10^{17} \mathrm{~cm}^{-3}$. The spectrum broadens significantly as the intensity is increased. A broad spectral feature is characteristic of moving from the weakly 

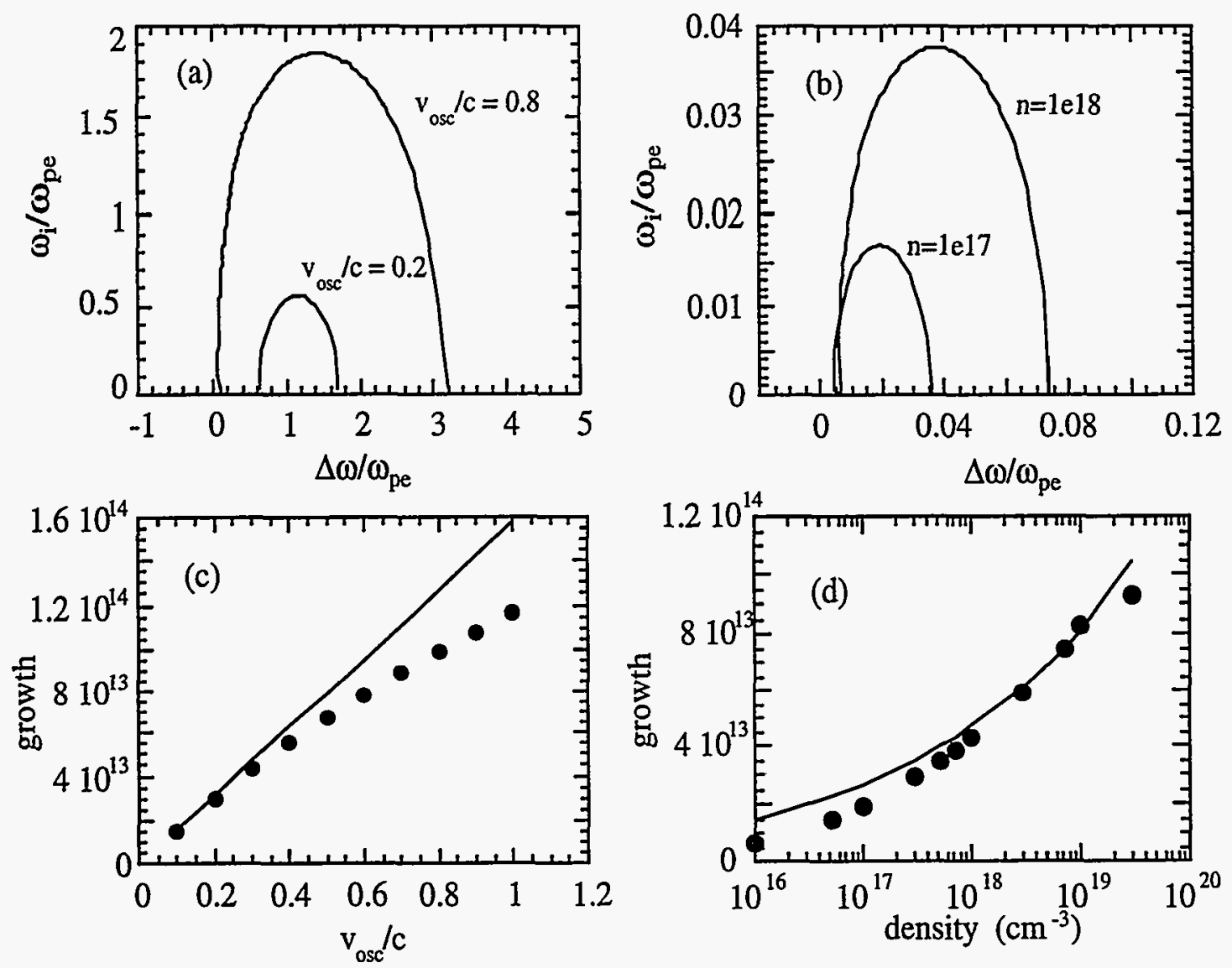

Figure 2.7: (a) Numerical spectra for $\mathrm{v}_{\mathrm{osc}} / \mathrm{c}=0.8$ and 0.2 , (b) numerical spectra for $\mathrm{n}_{0}=1 \times 10^{17}$ and $1 \times 10^{18} \mathrm{~cm}^{-3}$, and the growth rate of backscattered stimulated Raman scattering as a function of intensity (c) and density (d).

coupled to the strongly coupled regime. Figure 2.7(b) shows two spectra obtained at a fixed intensity $\left(\mathrm{v}_{\text {osc }} / \mathrm{c}=0.3\right)$ but different densities $\left(\mathrm{n}_{0}=1 \times 10^{17}, 1 \times 10^{18} \mathrm{~cm}^{-3}\right)$. The shape of the spectra are very similar, but the locations of the maxima are different, as expected based on the frequency matching condition (2.1). The growth rate, $\omega_{i}$, of backscattered SRS is plotted in figure 2.7 as a function of incident laser intensity (c) and plasma density (d) (solid circles). For figure 2.7(c), a fixed density of $\mathrm{n}_{0}=$ $1 \times 10^{18} \mathrm{~cm}^{-3}$ was used. A fixed intensity of $v_{o s c} / \mathrm{c}=0.3$ was used to generate figure 2.7(d). In both figures, the solid curve was generated using the growth rate, $\gamma_{0}$ (equation (2.68)). The analytic growth rate overestimates the growth at the higher 
intensities. At these intensities, however, the instability is probably in the strongly coupled regime and that growth rate (equation (2.69)) should be used. The growth rate, $\gamma_{0}$, agreed with the calculated growth, $\omega_{\mathrm{i}}$, over the density range studied, except for the lower densities where it overestimated the growth by less than a factor of 2 .
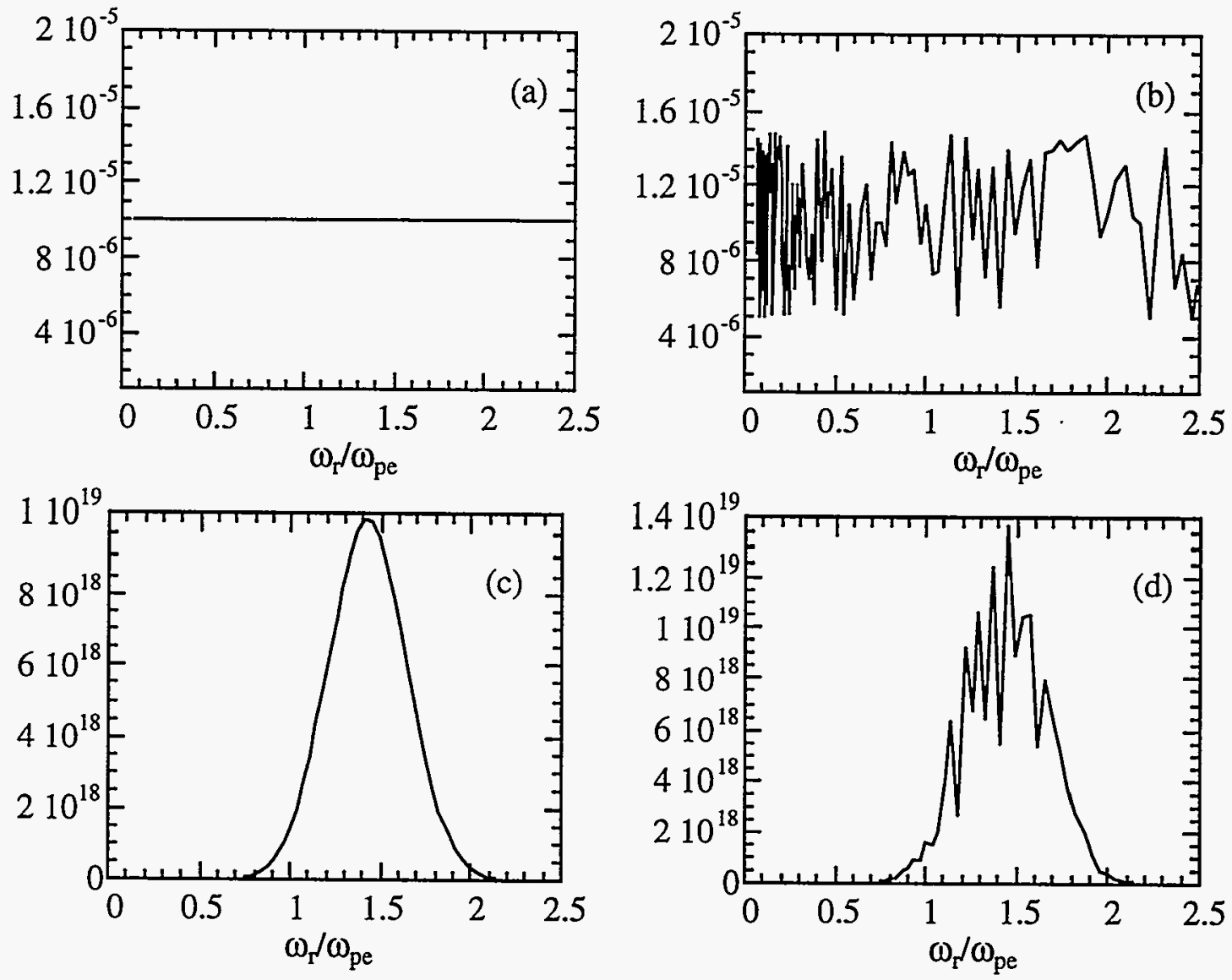

Figure 2.8: (a) Noise source used to obtain spectrum (c); (b) random noise source used to generate spectrum (d). For both spectra, $v_{\text {osc }} / c=0.8$ and $n_{0}=7 \times 10^{17} \mathrm{~cm}^{-3}$.

The noise source from which the electron plasma wave and scattered light wave of SRS grow can affect the actual shape of the spectrum obtained with the dispersion relation since the spectrum is simply an amplification of the initial noise level, as seen in the solution of the coupled equations in section 2.2. Therefore, the influence of the noise source on the expected frequency spectrum is discussed here. In obtaining the spectrum shown in figure $2.8(\mathrm{c}), \omega_{\mathrm{r}}$ is plotted versus we $\mathrm{e}^{\omega_{\mathbf{i}} \tau}$, where $\mathrm{w}$ is the noise source 
from which the SRS grew. In figure $2.8(\mathrm{a}), \mathrm{w}=1 \times 10^{-5}$. If this noise source were not uniform, i.e., $w=f\left(\omega_{r}\right)$, this would be reflected in the spectrum. This is illustrated in figure 2.8(d) where the noise source, $w$, used to obtain this spectrum was random in frequency space. This random noise source is shown in figure 2.8(b). Clearly, when SRS grows from a random noise source, the resulting spectrum is modified.

The spectra obtained with the dispersion relation can be used to predict the width and location of the SRS feature. Since $\omega_{\mathrm{e}}=\omega_{\mathrm{r}}$, the matching condition (2.1) can be used to obtain a location of the scattered feature by plotting we $e^{\omega_{\mathrm{i}} \tau}$ versus $\omega_{0}-\omega_{\mathrm{r}}$. Short pulse considerations [34], however, lead to a correction to this expression. When short pulse, high intensity lasers are used to drive stimulated Raman scattering in underdense plasmas, the driven modes exponentiate from the leading edge backward through the laser pulse. Since the plasma length $\left(L_{\text {plasma }}\right)$ is larger than the laser pulse length ( $c \tau$ ) for a short laser pulse, the backscattered light wave exits the laser pulse before it leaves the plasma. Therefore, it must couple to a normal mode in the undriven plasma behind the pulse. At high laser intensities, a coupling between two dissimilar dispersive media (i.e., inside the laser pulse where dispersion occurs and behind the laser pulse where ordinary dispersion is obeyed) must occur for the backscattered light wave to exit the excitation region. Since the laser pulse is moving, a moving dielectric boundary is created across which the phase of the scattered light wave must be matched. This matching leads to an expression for the "detected" frequency, $\omega_{\text {det }}$, given by

$$
\omega_{\mathrm{det}} \approx \omega_{0}-\frac{1}{2}\left(\delta+\omega_{\mathrm{r}}\right)
$$

where $\delta=\mathrm{c}\left(2 \mathrm{k}_{0}-\mathrm{k}_{\mathrm{e}}\right)$. This correction to the spectra calculated using the dispersion relation is illustrated in figure 2.9. The detected spectrum exhibits less of a red shift (relative to the laser frequency, 0 on this plot) than the uncorrected spectrum. In fact, in the detected spectrum, a significant quantity of energy is shifted by less than the plasma frequency, $\omega_{\text {pe. }}$. The detected spectrum is also broader. For low incident laser intensities $\left(\leq 10^{17} \mathrm{~W} / \mathrm{cm}^{2}\right)$, this correction is negligible and $\omega_{\mathrm{r}}$ accurately predicts the location of the spectrum. 


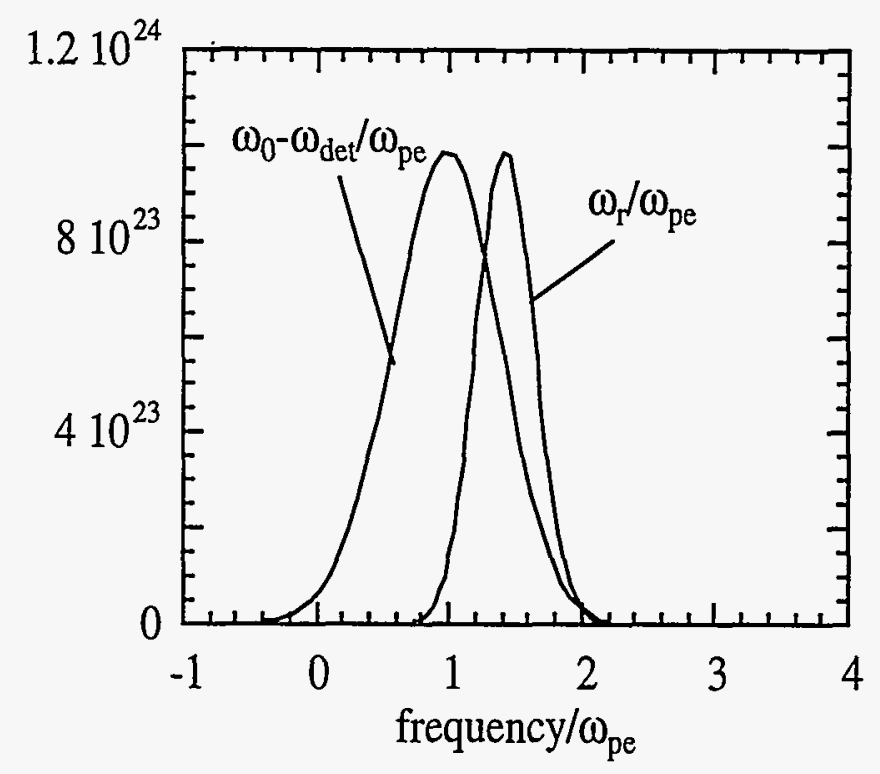

Figure 2.9: "Detected" spectrum obtained using $\omega_{0}-\omega_{\text {det }} / \omega_{\mathrm{pe}}$ and the uncorrected spectrum $\omega_{\mathrm{r}} / \omega_{\text {pe }}$. In both cases, $\mathrm{v}_{\mathrm{osc}} / \mathrm{c}=0.8$ and $\mathrm{n}_{0}=7 \times 10^{17} \mathrm{~cm}^{-3}$.

The validity of neglecting the anti-Stokes term (as nonresonant) to obtain equation (2.66) can be examined by comparing the solutions of equations (2.65) and (2.66). Figure 2.10 shows both solutions for the same parameters, i.e., $\mathrm{v}_{\mathrm{osc}} / \mathrm{c}=0.5$ and $\mathrm{n}_{0}=1 \times 10^{18} \mathrm{~cm}^{-3}$. The solid line shows the result obtained by solving the sixth order polynomial which results from equation (2.65) and the dashed line shows the fourth order result. The two curves are very similiar for this set of parameters. The obvious difference between the two solutions is that the fourth order solution does not grow to the same level the sixth order solution does (difference of 10-15\%). For most of the parameters considered here, however, solving the fourth order dispersion relation provides a sufficient description of the backscatter problem.

\section{Forward Scatter}

The dispersion relation, (2.65) can also be used to examine stimulated Raman forward scatter. For forward scatter, $\mathrm{k} \approx 0$ so both the Stokes and anti-Stokes terms can be resonant. Therefore, the dispersion relation as given by equation (2.65) must be 


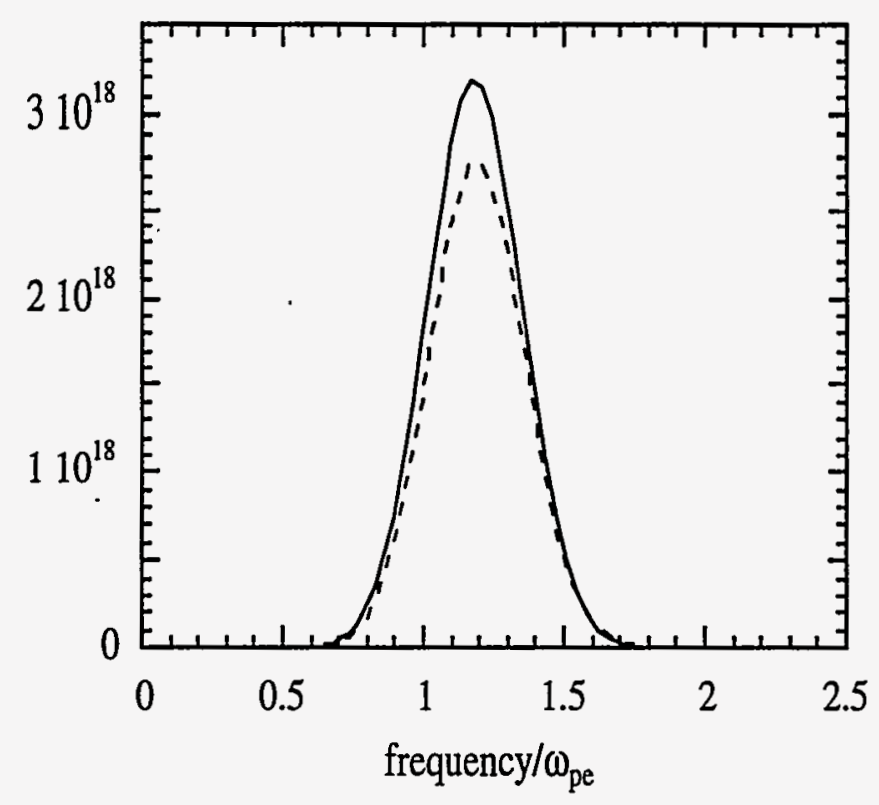

Figure 2.10: Comparison of solutions of the dispersion relation when the anti-Stokes term is neglected (dashed line) and included (solid line).

solved to predict the behavior of forward SRS.

The growth rate for forward SRS is obtained by first letting $\omega=\omega_{\mathrm{e}}+\delta \omega$. This allows $\mathrm{D}\left(\omega \pm \omega_{0}, \mathrm{k} \pm \mathrm{k}_{0}\right)$ to be written as

$$
\begin{aligned}
\mathrm{D}\left(\omega \pm \omega_{0}, \mathrm{k} \pm \mathrm{k}_{0}\right) & =\left[\left(\omega_{e} \pm \omega_{0}\right)^{2}-c^{2}\left(k \pm k_{0}\right)^{2}-\omega_{p e}^{2}\right]+2 \delta \omega\left(\omega_{e} \pm \omega_{0}\right) \\
& =2 \delta \omega\left(\omega_{e} \pm \omega_{0}\right)
\end{aligned}
$$

since growth is maximized when the scattered light waves are also resonant. Substituting this into equation (2.65) gives

$$
\omega_{\mathrm{e}}(\delta \omega)^{2}=\frac{1}{16} \omega_{\mathrm{pe}}{ }^{2} \mathrm{k}^{2} \mathrm{v}_{\mathrm{osc}}{ }^{2}\left(\frac{2 \omega_{\mathrm{e}}}{\omega_{\mathrm{e}}{ }^{2}-\omega_{0}^{2}}\right)
$$

Since $\delta \omega=\mathrm{i} \gamma_{0}$, this reduces to

$$
\gamma_{0}=\frac{1}{2 \sqrt{2}} \omega_{\mathrm{pe}}^{2}\left(\frac{\mathrm{v}_{\mathrm{osc}}}{\mathrm{c}}\right) \frac{1}{\omega_{0}}
$$

assuming $\omega_{\mathrm{e}} \ll \omega_{0}$ and $\mathrm{k}=\omega_{\mathrm{pe}} / \mathrm{c}$. This is the growth rate for forward SRS. It should be noted that this growth rate is a factor of $\sqrt{2}\left(\omega_{\mathrm{pe}} / \omega_{0}\right)^{\frac{3}{2}}$ times smaller than the 
growth rate for backscattered SRS (see equation (2.68)). I.e.,

$$
\gamma_{\mathrm{FS}}=\frac{1}{\sqrt{2}}\left(\frac{\omega_{0}}{\omega_{\mathrm{pe}}}\right)^{\frac{3}{2}} \gamma_{\mathrm{BS}}
$$

When driven by a short pulse, however, forward SRS can be a significant process since for forward SRS the growth is determined by the length of time the laser propagates through the plasma, $\tau_{\mathrm{p}}$, while for backscattered SRS the growth time is limited by the one-half the length of the laser pulse, $0.5 \tau$. I.e., for $\mathrm{v}_{\mathrm{osc}} / \mathrm{c}=0.4, \mathrm{n}_{0}=1 \times 10^{19} \mathrm{~cm}^{-3}$, $\tau=600 \mathrm{fsec}$, and $\tau_{\mathrm{p}}=3000 \mathrm{fsec}$, the growth of forward SRS is $\gamma_{\mathrm{FS}} \tau_{\mathrm{p}}=7.6$ while the growth of backscattered SRS is $0.5 \gamma_{\mathrm{BS}} \tau=34$.

To solve equation (2.65), it is rewritten as a sixth order polynomial equation in $\omega$. This polynomial, given by

$$
a_{6} \omega^{6}+a_{5} \omega^{5}+a_{4} \omega^{4}+a_{3} \omega^{3}+a_{2} \omega^{2}+a_{1} \omega+a_{0}=0
$$

can be solved for the roots. The coefficients are given by

$$
\begin{aligned}
& a_{6}=1 \\
& a_{5}=0 \\
& a_{4}=-2 c^{2} k^{2}+4 \omega_{0}^{2}+\omega_{e}^{2} \\
& a_{3}=8 c^{2} k k_{0} \omega_{0} \\
& a_{2}=c^{4} k^{4}-4 c^{4} k^{2} k_{0}^{2}+2 c^{2} k^{2} \omega_{e}^{2}+4 \omega_{0}^{2} \omega_{e}^{2}-\frac{1}{2} \omega_{p e}{ }^{2} k^{2} v_{\text {osc }}^{2} \\
& a_{1}=-8 c^{2} k k_{0} \omega_{0} \omega_{e}^{2} \\
& a_{0}=\frac{1}{2} \omega_{p e}{ }^{2} c^{2} k^{4} v_{\text {osc }}{ }^{2}-\omega_{e}^{2}\left(c^{4} k^{4}-4 c^{4} k^{2} k_{0}^{2}\right)
\end{aligned}
$$

This polynomial gives 4 real roots (representing electromagnetic waves) and 2 complex conjugate roots (representing the electron plasma wave). The six roots obtained from this polynomial are plotted in figure 2.11 for both the undriven $\left((\mathrm{a}) \mathrm{v}_{\mathrm{osc}} / \mathrm{c}=0\right.$, $\left.\mathrm{n}_{0}=1 \times 10^{19} \mathrm{~cm}^{-3}\right)$ and driven $\left((\mathrm{b}) \mathrm{v}_{\text {osc }} / \mathrm{c}=0.7, \mathrm{n}_{0}=1 \times 10^{19} \mathrm{~cm}^{-3}\right)$ cases. Curves $a, b, c$, and $d$ are the scattered light waves and curves e and $f$ are the electron plasma waves. Clearly, the coupling occurs for $\mathrm{k} / \mathrm{k}_{0} \approx 0$. 

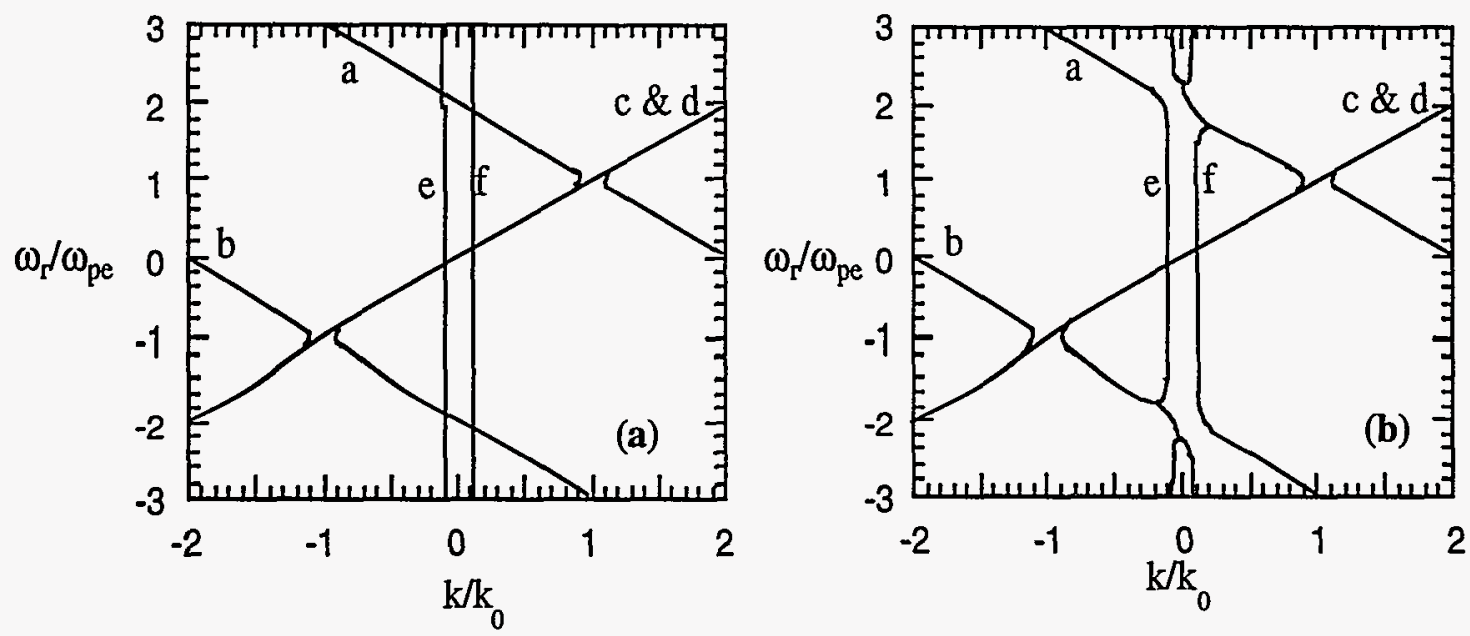

Figure 2.11: Roots of the (a) undriven $\left(\mathrm{v}_{\mathrm{osc}} / \mathrm{c}=0\right)$ and $(\mathrm{b})$ driven $\left(\mathrm{v}_{\mathrm{osc}} / \mathrm{c}=0.7\right)$ sixth order dispersion relation. For both cases, $\mathrm{n}_{0}=1 \times 10^{19} \mathrm{~cm}^{-3}$.

As is the case for stimulated Raman backscatter, plotting $\mathrm{e}^{\omega_{\mathrm{i}} \tau}$ versus $\omega_{\mathrm{r}}$ gives the power spectrum of the scattered light. Figure 2.12 shows a forward scatter spectrum obtained for $v_{\text {osc }} / c=1$ and $n_{0}=2 \times 10^{19} \mathrm{~cm}^{-3}$. There are two features of this spectrum which are distinctly different from the spectra obtained for backscattered SRS (figure 2.6(b)). First, there are two SRS features: a Stokes feature, located near +1 in this plot, and an anti-Stokes feature, located near -1 . The presence of both features implies that solving the fourth order polynomial, as is done for backscatter, is not adequate since the fourth order equation neglects a term which can be resonant for forward scatter. It should be noted that more than one Stokes and anti-Stokes feature can be present in the forward SRS spectrum, but this form of the dispersion relation neglects all but the first order of each. To produce the higher order modes, higher order terms must be retained in equation (2.65). The second difference between the backscattered and forward scattered spectra is that the absolute growth, $\omega_{\mathrm{i}}$, is much less for forward scatter than was observed for backscatter, implying that the threshold for experimental observation of forward SRS will be higher than it is for Raman backscatter.

The power spectrum varies with intensity, as shown in figure $2.13(\mathrm{a})$. In this 


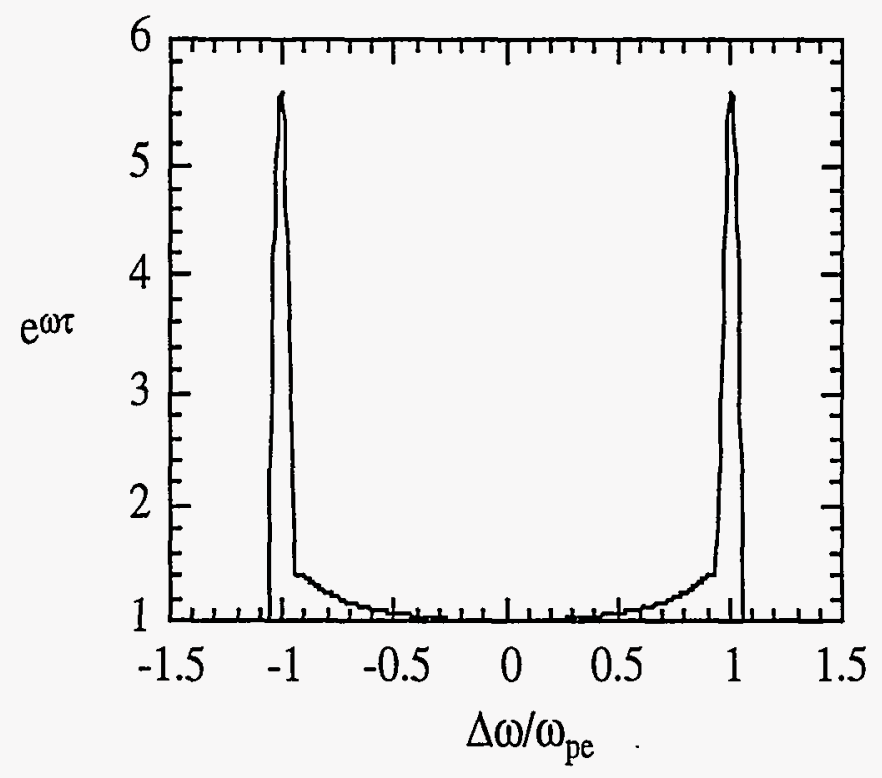

Figure 2.12: Spectrum obtained from the sixth order dispersion relation for forward stimulated Raman scattering. In this case, $\mathrm{v}_{\mathrm{osc}} / \mathrm{c}=1$ and $\mathrm{n}_{0}=2 \times 10^{19} \mathrm{~cm}^{-3}$.

plot, $\mathrm{e}^{\omega_{\mathrm{i}} \tau}$ is plotted versus $\omega_{\mathrm{r}} / \omega_{0}$ for $\mathrm{v}_{\text {osc }} / \mathrm{c}=0.5$ (solid) and 0.8 (dashed) (fixed $\left.\mathrm{n}_{0}=1 \times 10^{19} \mathrm{~cm}^{-3}\right)$. Both the Stokes and anti-Stokes features broaden as the intensity increases. The imaginary part of the frequency, $\omega_{i}$, is plotted as a function of intensity in figure 2.13(c) (solid triangles). This plot illustrates the dependence of the growth of forward SRS on incident laser intensity. The plasma density was fixed at $\mathrm{n}_{0}=1 \times 10^{19} \mathrm{~cm}^{-3}$. The solid line was obtained using the analytic expression for the growth rate, $\gamma_{0}$, equation (2.77). The two results agreed over the entire intensity range studied.

The calculated power spectrum also depends on density. In figure $2.13(\mathrm{~b}), \mathrm{e}^{\omega_{\mathrm{j}} \tau}$ is plotted for $\mathrm{n}_{0}=7 \times 10^{18} \mathrm{~cm}^{-3}$ (solid curve) and $\mathrm{n}_{0}=2 \times 10^{19} \mathrm{~cm}^{-3}$ (dashed curve) for a fixed intensity of $\mathrm{v}_{\mathrm{osc}} / \mathrm{c}=0.8$. Both the Stokes and anti-Stokes modes shifted as the density increased, as expected. The growth rate, given by $\omega_{\mathrm{i}}$, is plotted as a function of plasma density in figure 2.13(d) (solid triangles). The incident intensity was fixed at $\mathrm{v}_{\text {osc }} / \mathrm{c}=0.8$. The analytic expression for the growth rate (equation (2.77)) was used to obtain the solid curve. Again, agreement is observed between the two solutions. 

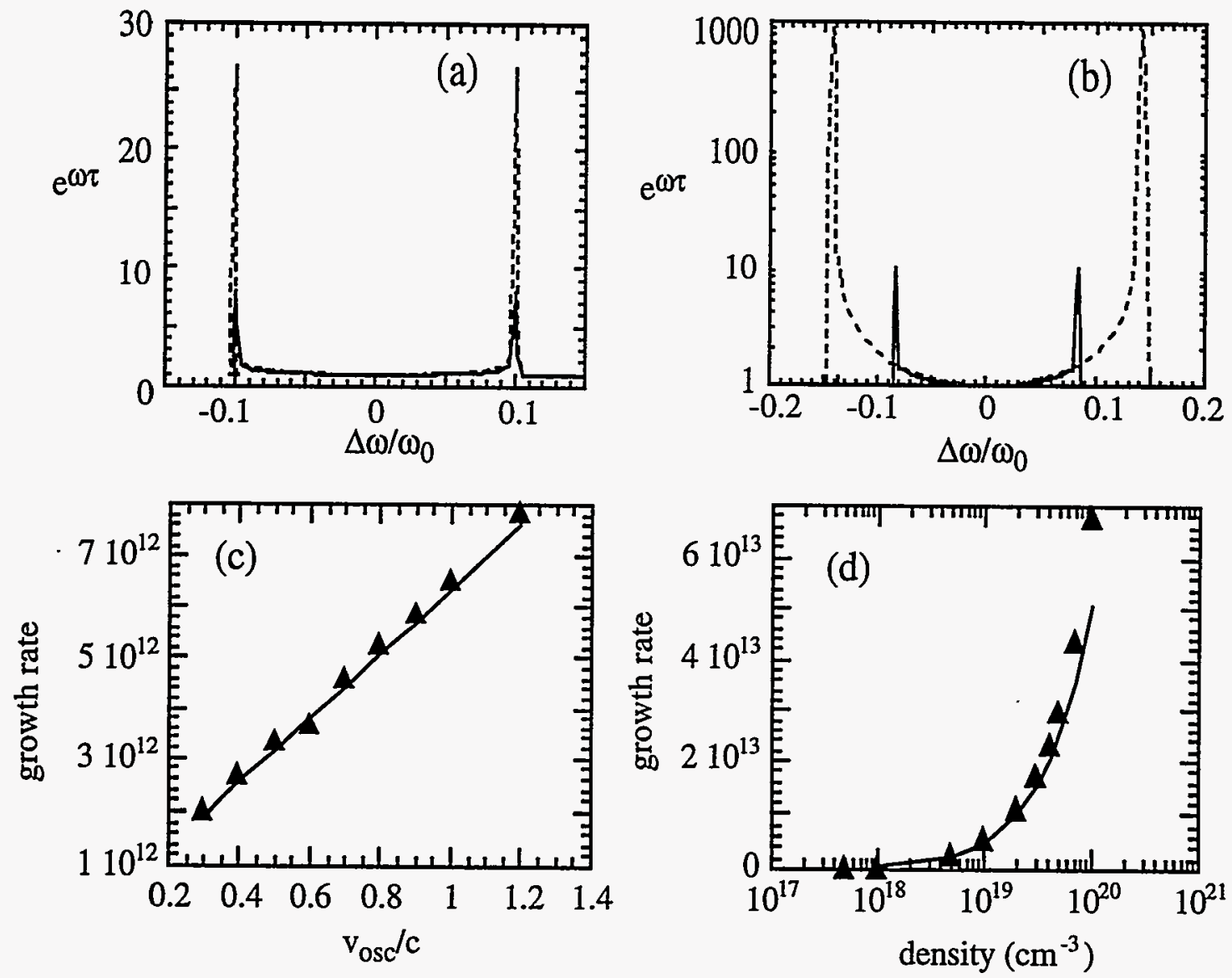

Figure 2.13: (a) Spectrum of forward SRS for $\mathrm{v}_{\text {osc }} / \mathrm{c}=0.5$ (solid) and 0.8 (dashed); (b) forward SRS spectrum for $n_{0}=7 \times 10^{18} \mathrm{~cm}^{-3}$ and $2 \times 10^{19} \mathrm{~cm}^{-3}$; (c) growth as a function of incident intensity (solid triangles); and (d) growth as a function of plasma density (solid triangles). In plots (c) and (d), the solid line was obtained using equation (2.77).

The location of the scattered SRS features can be obtained by plotting $\mathrm{e}^{\omega_{\mathbf{5}} \tau}$ versus $\omega_{0}-\omega_{\mathrm{r}}$. For forward SRS, there is no correction necessary (until the instability saturates and nonlinear effects become important) to account for short pulse laser effects since the scattered light wave travels in the same direction as the pump wave in forward Raman scattering. Therefore, there is no phase matching that must be made across a moving dielectric boundary as for backscatter and the preceding formalism, as stated, can be used to predict the forward Raman spectra (assuming no saturation). 


\subsubsection{Relativistic}

The derivation of the relativistic dispersion relation is more complicated than the derivation of the nonrelativistic version due to the dependence of the relativistic "correction factor", $\gamma$, on the velocity. This derivation is described elsewhere ([38], [80]) and is discussed here for completeness. The relativistic dispersion relation is given by

$$
1=\frac{\mathrm{v}_{\mathrm{osc}}{ }^{2} \omega_{\mathrm{pe}}{ }^{2}}{4 \mathrm{c}^{2} \gamma^{3}}\left[\frac{\mathrm{c}^{2} \mathrm{k}^{2}}{\omega^{2}-\omega_{\mathrm{e}}{ }^{2}}\right]\left[\frac{1}{\mathrm{D}\left(\omega-\omega_{0}, \mathrm{k}-\mathrm{k}_{0}\right)}+\frac{1}{\mathrm{D}\left(\omega+\omega_{0}, \mathrm{k}+\mathrm{k}_{0}\right)}\right]
$$

where $\gamma=\sqrt{1+\mathrm{vosc}^{2} / \mathrm{c}^{2}}, \omega_{\mathrm{e}}^{2}=\omega_{\mathrm{pe}}{ }^{2} / \gamma+3 \mathrm{k}^{2} \mathrm{v}_{\mathrm{th}}{ }^{2}$, and $\mathrm{D}(\omega, \mathrm{k})=\omega^{2}-\mathrm{c}^{2} \mathrm{k}^{2}-\omega_{\mathrm{pe}}{ }^{2} / \gamma$.

\section{Backscatter}

As was the case with the nonrelativistic dispersion relation, the anti-Stokes term, $\left(\left(\omega+\omega_{0}\right)^{2}-c^{2}\left(\mathrm{k}+\mathrm{k}_{0}\right)^{2}-\omega_{\mathrm{pe}}{ }^{2} / \gamma\right)^{-1}$, can be neglected for stimulated Raman backscatter since it is very nonresonant. Therefore, equation (2.86) can be rewritten as

$$
1=\frac{\mathrm{v}_{\mathrm{osc}}{ }^{2} \omega_{\mathrm{pe}}{ }^{2}}{4 \mathrm{c}^{2} \gamma^{3}}\left[\frac{\mathrm{c}^{2} \mathrm{k}^{2}}{\omega^{2}-\omega_{\mathrm{e}}^{2}}\right]\left[\frac{1}{\mathrm{D}\left(\omega-\omega_{0}, \mathrm{k}-\mathrm{k}_{0}\right)}\right]
$$

The relativistic version of the growth rate for stimulated Raman backscatter can be obtained from equation (2.68) by making the substitutions $\mathrm{v}_{\text {osc }} / c \rightarrow(1 / \gamma) \mathrm{v}_{\text {osc }} / c$, $\omega_{\mathrm{pe}} \rightarrow(1 / \sqrt{\gamma}) \omega_{\mathrm{pe}}$, and $\omega_{\mathrm{e}} \rightarrow(1 / \sqrt{\gamma}) \omega_{\mathrm{e}} \cdot[37]$ This gives

$$
\gamma_{0 \mathrm{r}}=\frac{\mathrm{kv}_{\mathrm{osc}}}{4 \gamma^{\frac{5}{4}}}\left[\frac{\omega_{\mathrm{pe}}^{2}}{\omega_{\mathrm{e}}\left(\omega_{0}-\omega_{\mathrm{e}}\right)}\right]^{\frac{1}{2}}
$$

for the relativistic growth rate of backscattered SRS. The relativistically correct strongly coupled growth rate can be similarly obtained and is given by

$$
\gamma_{\mathrm{scr}}=\frac{\sqrt{3}}{2} \frac{1}{\gamma}\left[\frac{\omega_{\mathrm{pe}}{ }^{2} \mathrm{k}^{2} \mathrm{v}_{\mathrm{osc}}{ }^{2}}{\omega_{0}}\right]^{\frac{1}{3}} .
$$

To solve for the roots of equation (2.87), it is rewritten as a fourth order polynomial.

$$
a_{4} \omega^{4}+a_{3} \omega^{3}+a_{2} \omega^{2}+a_{1} \omega+a_{0}=0
$$


The coefficients are given by

$$
\begin{aligned}
& a_{4}=1 \\
& a_{3}=-2 \omega_{0} \\
& a_{2}=2 c^{2} k k_{0}-c^{2} k^{2}-\omega_{e}^{2}+\frac{\mathrm{vosc}^{2} \omega_{\mathrm{pe}}}{4 \mathrm{c}^{2} \gamma^{3}} \\
& a_{1}=2 \omega_{0} \omega_{\mathrm{e}}^{2} \\
& \mathrm{a}_{0}=\omega_{\mathrm{e}}^{2}\left(\mathrm{c}^{2} \mathrm{k}^{2}-2 \mathrm{c}^{2} \mathrm{kk}_{0}-\frac{\mathrm{v}_{\mathrm{osc}}{ }^{2} \omega_{\mathrm{pe}}}{4 \mathrm{c}^{2} \gamma^{3}}\right)-\frac{\mathrm{v}_{\text {osc }}{ }^{2} \omega_{\mathrm{pe}}{ }^{2} k^{2}}{4 \gamma^{3}}
\end{aligned}
$$

This polynomial produces 2 real roots and 2 complex conjugate roots when solved

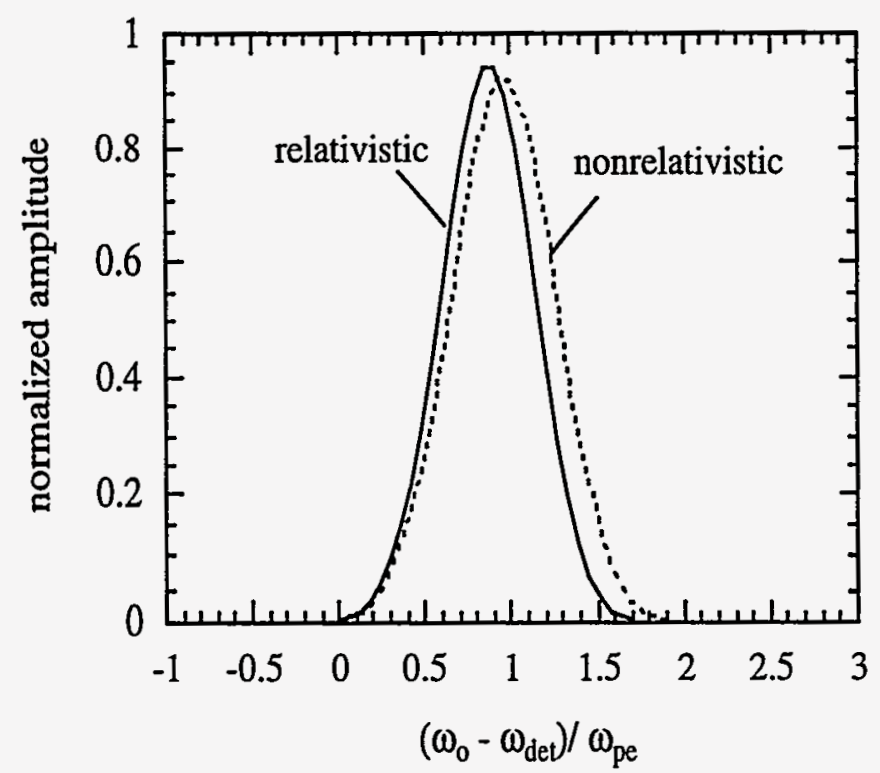

Figure 2.14: "Detected" spectrum obtained with the relativistic dispersion relation and correction (solid) and the nonrelativistic versions (dashed).

with $\mathrm{k} \approx 2 \mathrm{k}_{0}$. As was the case for the nonrelativistic dispersion relation, the real part of the frequency, $\omega_{r}$, gives information about the location of growth while the imaginary part, $\omega_{\mathrm{i}}$ is a measure of the growth. Figure 2.14 shows a power spectrum obtained for $\mathrm{v}_{\mathrm{osc}} / \mathrm{c}=0.5$ and $\mathrm{n}_{0}=1 \times 10^{18} \mathrm{~cm}^{-3}$. The solid line is the power spectrum obtained using a relativistic version of the "detected" frequency, to account for the short pulse considerations discussed earlier. The relativistically correct "detected" 
spectrum is given by

$$
\omega_{\mathrm{det}} \approx \omega_{0}-\frac{1}{2}\left(\delta+\frac{\omega_{\mathrm{r}}}{\sqrt{\gamma}}\right)
$$

The dashed line of figure 2.14 represents the spectrum generated from the nonrelativistic dispersion relation and the nonrelativistic "detected" frequency. Using the relativistic versions produces a slight additional shift. For low incident laser intensities, $\gamma \approx 1$ and there is no difference between the relativistically and nonrelativistically calculated spectra.

Figures 2.15(a) and 2.15(b) illustrate the variation in the growth of backscattered SRS as a function of incident intensity and plasma density respectively (solid circles). For figure 2.15(a), the density was fixed at $\mathrm{n}_{0}=1 \times 10^{18} \mathrm{~cm}^{-3}$ and for figure $2.15(\mathrm{~b})$, the incident laser intensity was restricted to $\mathrm{v}_{\mathrm{osc}} / \mathrm{c}=0.3$. The solid lines were obtained using the relativistic growth rate, $\gamma_{0 r}$, equation (2.88). The open triangles show the
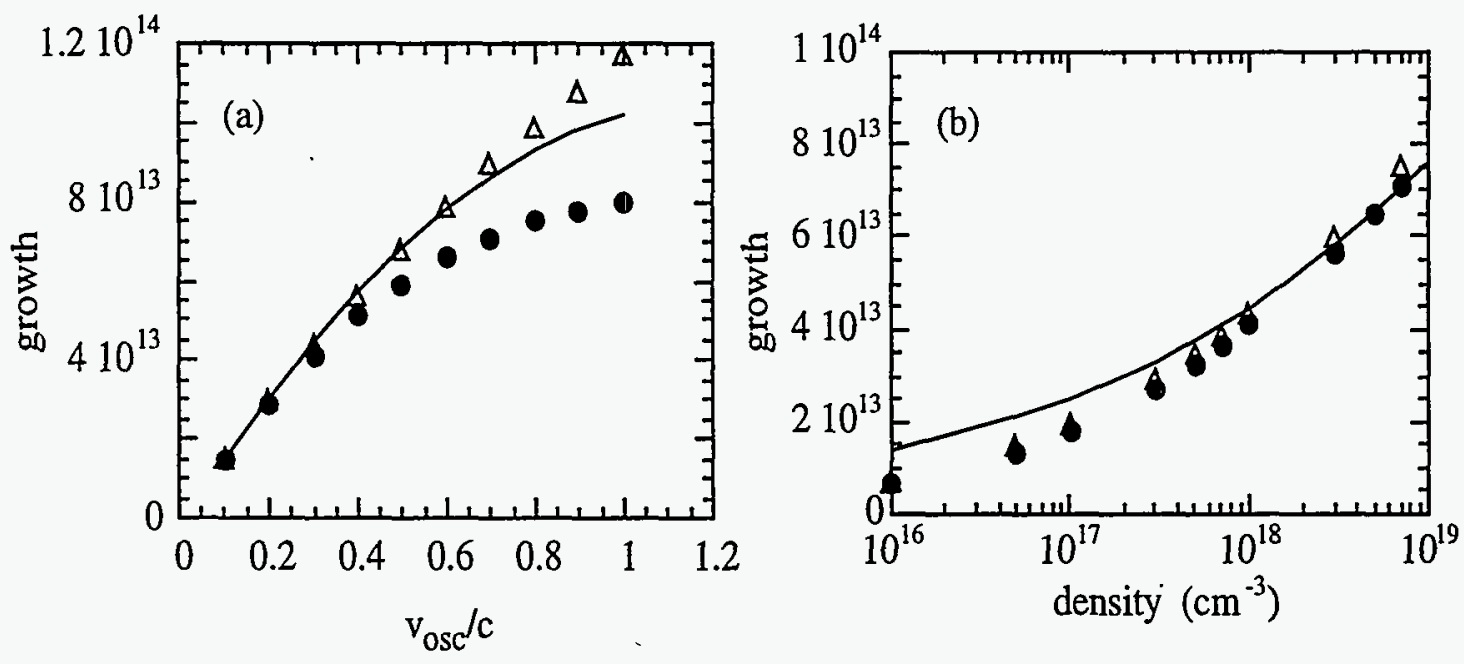

Figure 2.15: Growth of the plasma wave as a function of incident intensity (a) and plasma density (b). The results from the nonrelativistic calculation are shown as open triangles and the solid line was calculated using the relativistic growth rate (equation (2.88)).

results obtained with the nonrelativistic dispersion relation. The discrepancy between the analytic relativistic growth rate result and the dispersion relation prediction in 
figure 2.15(a) indicates that the strongly coupled growth rate is necessary. It is also clear from figure 2.15 (a) that for $\mathrm{v}_{\mathrm{osc}} / \mathrm{c}>0.4$, the relativistic correction to the dispersion relation becomes significant in the overall growth. The nonrelativistic dispersion relation predicts higher growth than the relativistic dispersion relation for these intensities. Therefore, the relativistic dispersion relation should be used. The predicted and analytic growth rates were in good agreement for most of the densities studied, as seen in figure 2.15(b). If the noise source is assumed to be random, the electron plasma wave and scattered light wave develop structure, as was observed in the solution of the nonrelativistic dispersion relation. (see figure 2.8)

\section{Forward Scatter}

The relativistic dispersion relation (2.86) can also be used to predict the behavior of forward stimulated Raman scattering. Both the Stokes and anti-Stokes terms can be resonant for forward SRS since $\mathrm{k} \approx 0$ for forward scatter. The relativistically correct growth rate for forward SRS is given by

$$
\gamma_{0 \mathrm{r}}=\frac{1}{2 \sqrt{2} \gamma^{2}} \frac{\omega_{\mathrm{pe}}{ }^{2}}{\omega_{0}} \frac{\mathrm{v}_{\mathrm{osc}}}{\mathrm{c}}
$$

This growth rate was obtained from equation (2.77) by making the substitutions $\mathrm{v}_{\mathrm{osc}} / \mathrm{c} \rightarrow(1 / \gamma)\left(\mathrm{v}_{\mathrm{osc}} / \mathrm{c}\right)$ and $\omega_{\mathrm{pe}} \rightarrow(1 / \sqrt{\gamma}) \omega_{\mathrm{pe}}$. The dispersion relation, $(2.86)$, can be written as a sixth order polynomial in $\omega$ which can be solved for the roots, as was done with the nonrelativistic dispersion relation.

$$
a_{6} \omega^{6}+a_{5} \omega^{5}+a_{4} \omega^{4}+a_{3} \omega^{3}+a_{2} \omega^{2}+a_{1} \omega+a_{0}=0
$$

The coefficients are given by

$$
\begin{aligned}
& a_{6}=1.0 \\
& a_{5}=0.0 \\
& a_{4}=4 \omega_{0}^{2}-2 c^{2} k^{2}-\omega_{\mathrm{e}}^{2}+\frac{\mathrm{vosc}^{2} \omega_{\mathrm{pe}}^{2}}{2 \mathrm{c}^{2} \gamma^{3}} \\
& \mathrm{a}_{3}=8 \mathrm{c}^{2} \mathrm{kk}_{0} \omega_{0}
\end{aligned}
$$




$$
\begin{aligned}
& \mathrm{a}_{2}=c^{2} \mathrm{k}^{2}\left(\mathrm{c}^{2} \mathrm{k}^{2}-4 \mathrm{c}^{2} \mathrm{k}_{0}^{2}-\frac{\mathrm{v}_{\mathrm{osc}}{ }^{2} \omega_{\mathrm{pe}}{ }^{2}}{\mathrm{c}^{2} \gamma^{3}}\right)+\omega_{\mathrm{e}}{ }^{2}\left(2 \mathrm{c}^{2} \mathrm{k}^{2}-4 \omega_{0}{ }^{2}-\frac{\mathrm{v}_{\mathrm{osc}}{ }^{2} \omega_{\mathrm{pe}}}{2 \mathrm{c}^{2} \gamma^{3}}\right)(2 \\
& \mathrm{a}_{1}=-8 \mathrm{c}^{2} \mathrm{kk}_{0} \omega_{0} \omega_{\mathrm{e}}{ }^{2} \\
& \mathrm{a}_{0}=\omega_{\mathrm{e}}{ }^{2}\left(4 \mathrm{c}^{4} \mathrm{k}^{2} \mathrm{k}_{0}{ }^{2}-\mathrm{c}^{4} \mathrm{k}^{4}+\frac{\mathrm{v}_{\mathrm{osc}}{ }^{2} \omega_{\mathrm{pe}}{ }^{2} \mathrm{k}^{2}}{2 \gamma^{3}}\right)+\frac{\mathrm{v}_{\mathrm{osc}}{ }^{2} \omega_{\mathrm{pe}}{ }^{2} \mathrm{c}^{2} \mathrm{k}^{2}}{2 \gamma^{3}}
\end{aligned}
$$

The solution to the polynomial (2.98) provides information on the growth and behav-

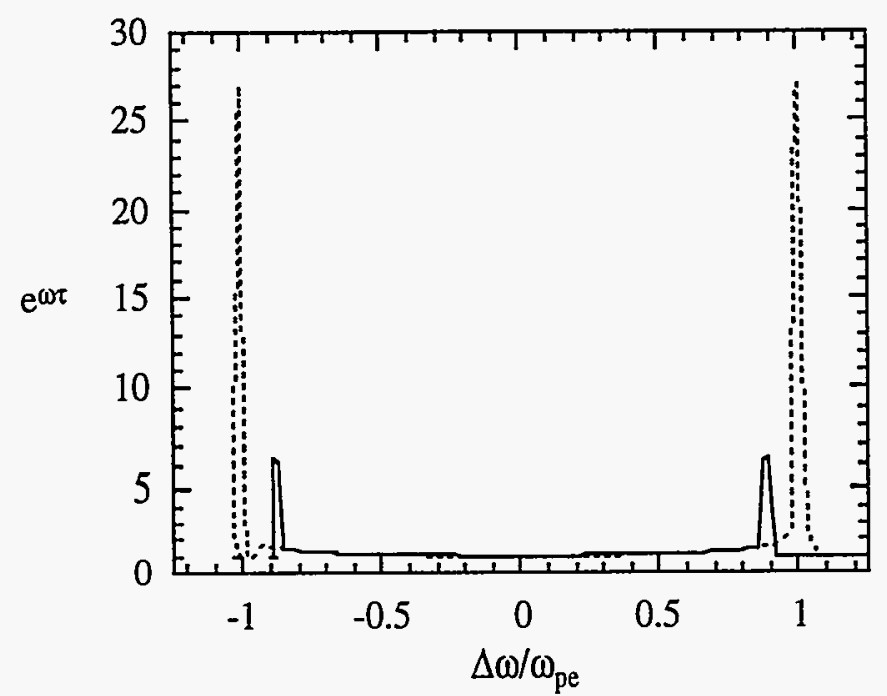

Figure 2.16: Forward SRS spectrum obtained by solving the relativistic dispersion relation (solid) and the nonrelativistic dispersion relation (dashed) for $\mathrm{n}_{0}=1 \times$ $10^{19} \mathrm{~cm}^{-3}$ and $\mathrm{v}_{\mathrm{osc}} / \mathrm{c}=0.8$.

ior of forward SRS. Just as when the nonrelativistic dispersion relation was solved, this relativistic version gives 4 real roots and 2 complex conjugate roots. Figure 2.16 shows the power spectrum obtained for $v_{\text {osc }} / c=0.8$ and $n_{0}=1 \times 10^{19} \mathrm{~cm}^{-3}$. The solid line is the spectrum obtained using the relativistic dispersion relation. The dashed line was produced with the nonrelativistic dispersion relation. Clearly for these parameters, the nonrelativistic disperion relation does not predict the appropriate locations or amplitude of the SRS features. The spectrum in forward SRS needs no 'short pulse correction' since in forward SRS the scattered light wave travels in the same direction as the pump wave.

The intensity dependence of the growth of the forward SRS instability is depicted 

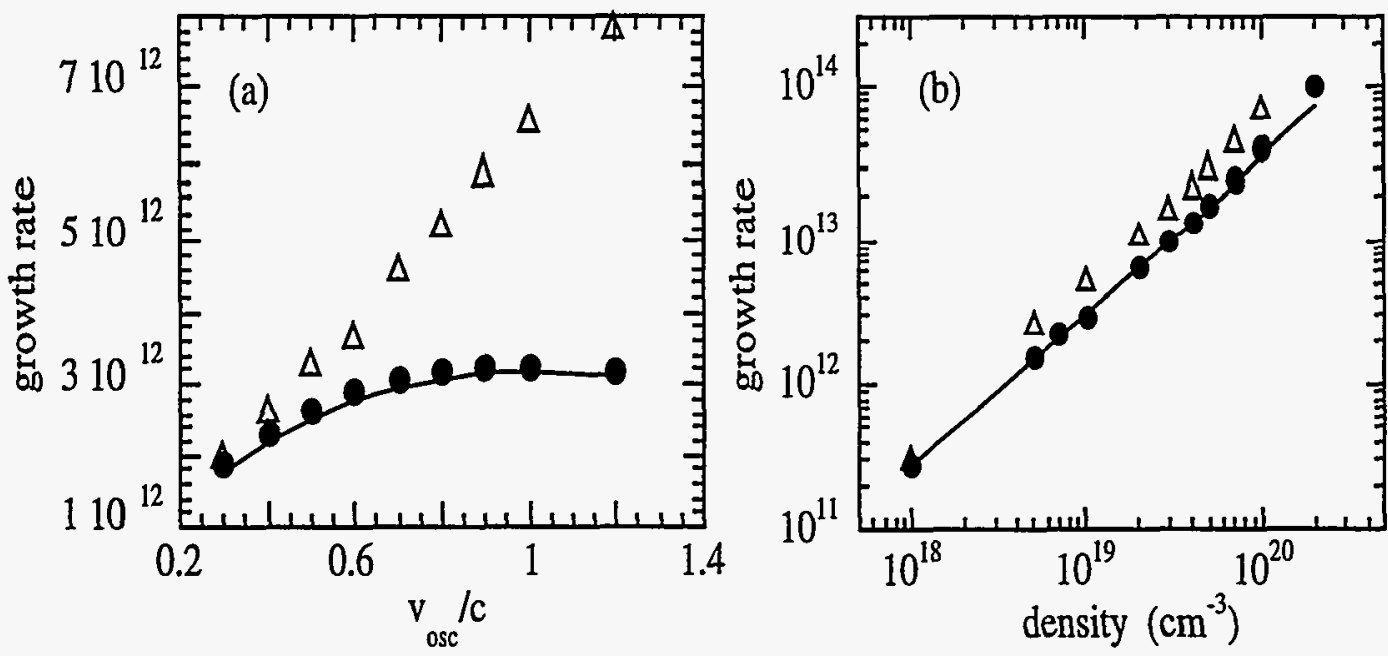

Figure 2.17: Incident intensity (a) and plasma density (b) dependence of the growth of forward SRS (solid circles). The results obtained with the nonrelativistic dispersion relation are shown as open triangles. The solid line was generated using equation (2.97).

in figure 2.17(a) as solid circles. The solid line was calculated using the analytic expression for the relativistic growth rate, equation (2.97). The open triangles show results obtained from the nonrelativistic dispersion relation. A fixed density of $\mathrm{n}_{0}=$ $1 \times 10^{19} \mathrm{~cm}^{-3}$ was used in these calculations. The growth predicted by the relativistic growth rate and the relativistic dispersion relation agreed over this parameter range. Clearly, however, for $v_{o s c} / c>0.4$, the relativistic dispersion must be used to correctly predict the growth. The nonrelativistic dispersion relation overestimates the total growth of the forward SRS for nearly all the intensities.

Figure 2.17(b) shows the density dependence of forward SRS growth. The incident intensity was fixed at $\mathrm{v}_{\mathrm{osc}} / \mathrm{c}=0.8$. The solid line was generated with the relativistic expression for the growth rate, equation (2.97). Good agreement was observed between the analytic and calculated results. The open triangles were obtained with the nonrelativistic dispersion relation. The nonrelativistic solution overestimates the growth for all the densities considered. 


\subsection{Summary}

In this chapter, the coupled equations describing the unstable growh of a density perturbation in the presence of a laser field were derived and solved for parameters relevant to the short pulse, high intensity laser underdense plasma interaction experiments of this work. The evolution of the electron plasma and scattered light waves was calculated as the laser propagated through the plasma. The growth of these waves was also determined. The relativistic and nonrelativistic dispersion relations were solved for both backscattered and forward scattered SRS to predict the growth and location of SRS features. The relativistic and nonrelativistic solutions were compared and the growth of the plasma wave was found as a function of density and intensity. The influence of a random noise source was also examined. These dispersion relations, and the information they provide about the SRS features, will be utilized in chapters 4 and 5 to model the experimental observations of forward scattered SRS and backscattered SRS. 


\section{Chapter 3}

\section{Experimental Apparatus and}

\section{Parameters}

In this chapter, the experimental apparatus, laser system, and diagnostics used to obtain the data are presented. An overview of the experimental setup is given first with details regarding the laser system and specific diagnostics presented later in the chapter. The diagnostics have been divided into two categories: light collection diagnositics and electron energy diagnostics. The creation of the plasma and the plasma parameters are also discussed.

\subsection{System Overview}

The experimental configurations used in this work are depicted in figure 3.1 and are intended to serve as a reference throughout the remainder of this work. Figure 3.1(a) illustrates the configuration used for the backscattered SRS experiments. The experimental setups used to investigate forward SRS and its associated fast electrons are shown in figures $3.1(\mathrm{~b}), 3.1(\mathrm{c})$, and $3.1(\mathrm{~d})$.

In both the forward scatter and backscatter SRS experiments, the laser light was relayed to the experimental vacuum chamber by a series of high reflectivity mirrors where it was focussed with a $69 \mathrm{~cm}(\mathrm{f} / 8.2)$ aspheric lens. The lens served a secondary 


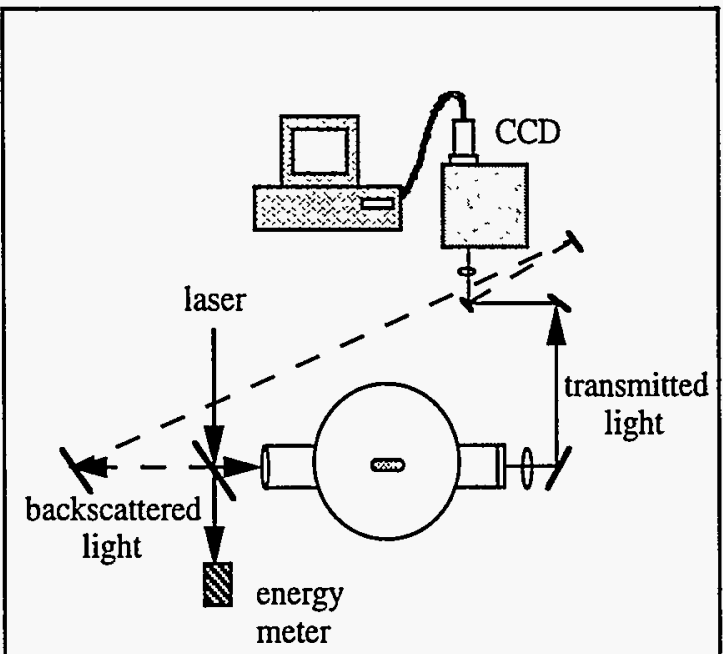

(a) Backscattered SRS experimental setup

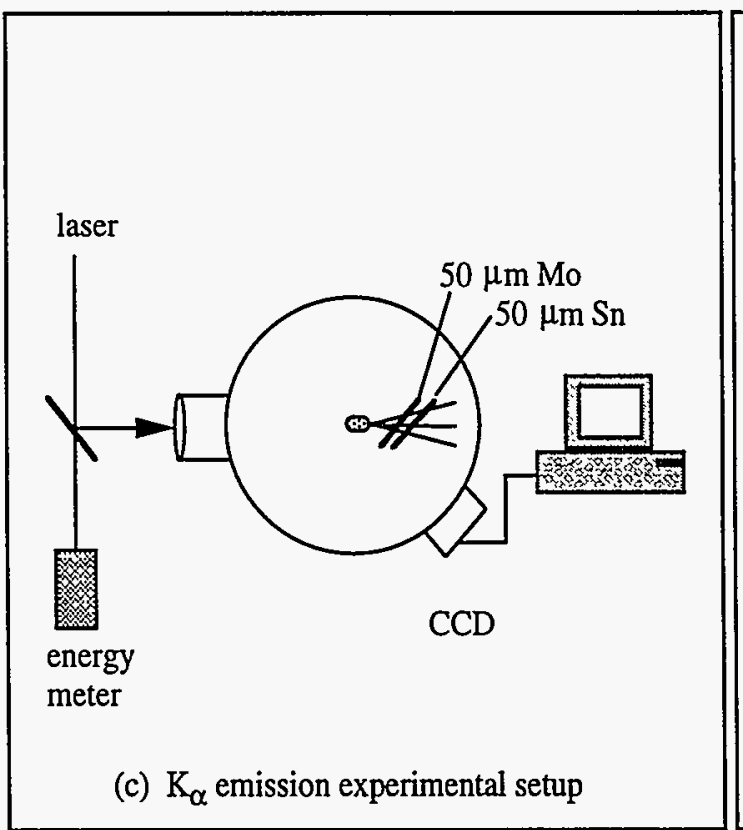

Figure 3.1: Experimental Configurations 
purpose as the vacuum window. Near chamber center, the focussing laser created and interacted with an underdense plasma. The plasmas were formed from either a static gas fill (figure 3.1(a)) or from a burst of gas from a pulsed valve (figures 3.1(b),(c),(d)). The leakage through the final turning mirror before the experimental chamber was directed to an absorbing calorimeter to provide a measurement of the energy entering the chamber. The energy transmitted through the chamber within the original focal cone was selectively monitored with a 4" absorbing glass calorimeter.

As shown in figure 3.1(a), the transmitted and directly backscattered light were collected and spectrally resolved. The transmitted energy and transmitted spectrum could not be simultaneously monitored because the calorimeter used in the transmitted energy measurement blocked the transmitted light path. Figure 3.1(b) shows the setup used to collect and spectrally resolve near-forward scattered light. Nearforward scattered light was collected in a narrow region $\left(5^{\circ}-15^{\circ}\right.$; just outside the vacuum focal cone) to improve signal levels of the relatively weak scattered light relative to the transmitted light. The energy transmitted in the vacuum focal cone was also monitored with a large aperture calorimeter in this configuration. The energy of electrons accelerated by the plasma wave was measured by two techniques. In one technique, depicted in figure 3.1(c), a metal target was placed several centimeters past the plasma region in the direct forward direction and the $\mathrm{K}_{\alpha}$ flourescence generated when energetic electrons struck this target was monitored. [53] In a second method, a $90^{\circ}$ imaging deflecting magnet $([76],[45])$ on loan from the UCLA Plasma Accelerator Group was placed seven centimeters past the plasma region and used to direct electrons onto a silicon surface barrier detector, as shown in figure 3.1(d). In this technique, a second surface barrier detector was used to monitor $\mathrm{x}$-ray emission from the plasma. 


\subsection{The Laser System}

The laser system used in these experiments is a Nd:glass tabletop terawatt system [89] based on the concept of chirped pulse amplification. ([77],[110]) Chirped pulse amplification (CPA), a technique originally used in radar systems, is a process in which a short laser pulse is "stretched" temporally, amplified, then recompressed into a short pulse. The application of this technique to lasers made possible the development of high peak power ( $\geq 1$ terawatt), short pulse ( $\leq 1 \mathrm{psec}$ ) lasers capable of very high intensities $\left(\geq 10^{18} \mathrm{~W} / \mathrm{cm}^{2}\right)$. Previously, the amplification of short pulses with solid state media was limited by the onset of filamentation due to the intensity dependent refractive index of the solid-state materials. [110] CPA circumvents this problem by amplifying long pulses, keeping the intensity in the amplifiers below the nonlinearly induced phase distortion level.

The laser system used in these experiments was developed by F. Patterson and M. Perry at the Lawrence Livermore National Lab. [89] A schematic of this system is shown in figure 3.2. The front end of this system is a titanium sapphire $\left(\mathrm{Ti}: \mathrm{Al}_{2} \mathrm{O}_{3}\right)$ laser pumped by 16 Watts from an argon ion laser. Ti:sapphire is broadband and can produce temporally short, clean pulses. Although Ti:sapphire has optimum perfomance near $800 \mathrm{~nm}$, it can oscillate at $1.0527 \mu \mathrm{m}$ with reduced gain. [111] The output from the Ti:sapphire system is a modelocked train of nearly transform limited $420 \mathrm{~mW}, 130 \mathrm{fsec}$ pulses operating with a repetition rate of $76 \mathrm{MHz}$. The bandwidth of the pulses is large, approximately $13 \mathrm{~nm}$. The pulses from the Ti:sapphire are Gaussian in both time and space. The 130 fsec pulses from the Ti:sapphire are stretched into $600 \mathrm{psec}, 30 \mathrm{~mW}$ pulses in a standard double pass stretcher configuration using a 1740 grooves $/ \mathrm{mm}$ grating and a $1.79 \mathrm{~m}$ lens. The bandwidth of the pulse gets 'chopped' in the stretcher by the lens (3" aperture) prior to the grating reducing the bandwidth of the seed pulse to $4.8 \mathrm{~nm}$.

The long pulse $(600 \mathrm{psec})$ propagates into a ring regenerative amplifier, where a single pulse from the train is selected with a Pockels cell. This is possible because the rise time for the Pockels cell is only a few nanoseconds and the pulses are separated 


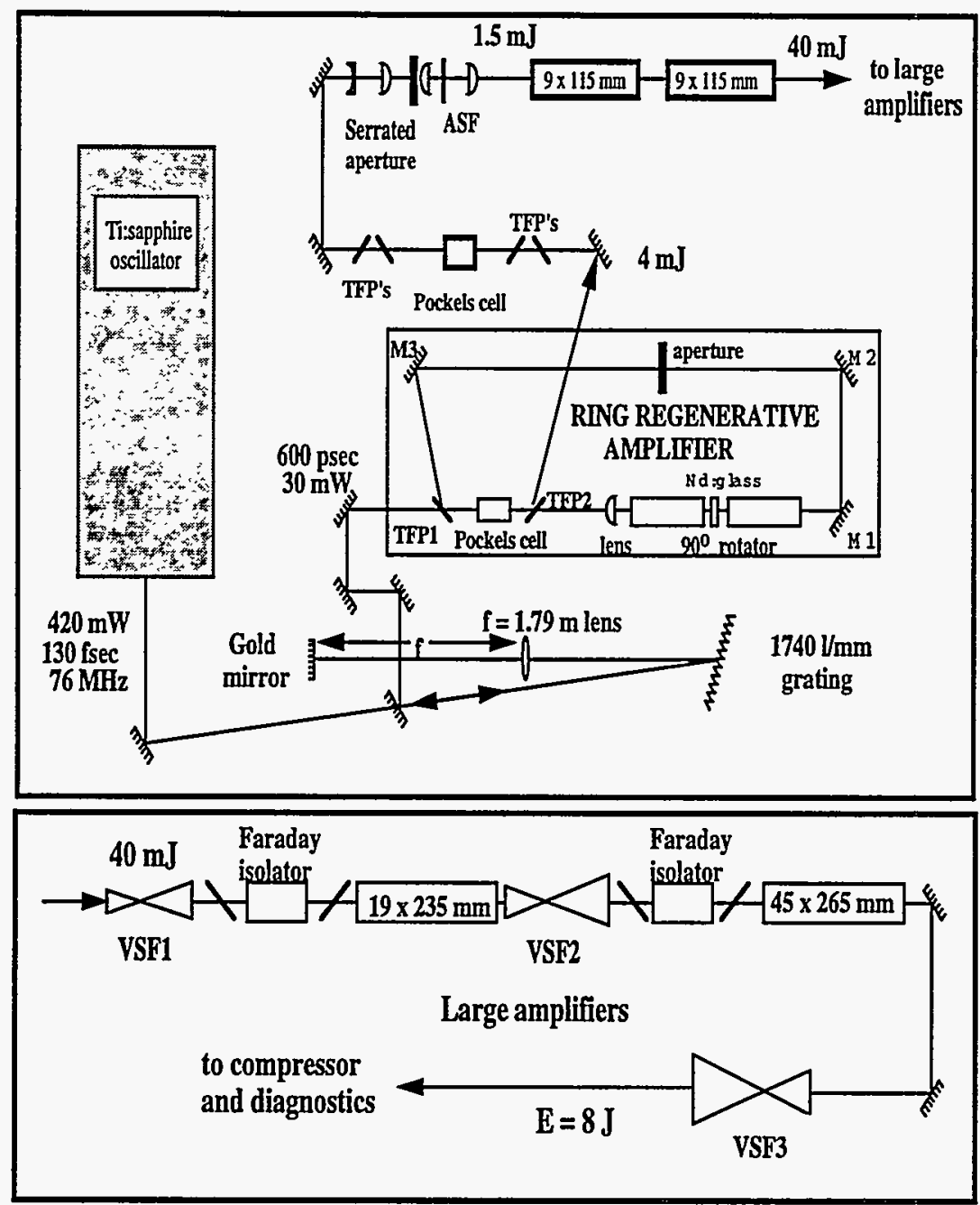

lass of compressor $=37 \%$

$\tau=600 \mathrm{fsec}$

$\mathrm{E}=5 \mathrm{~J}$

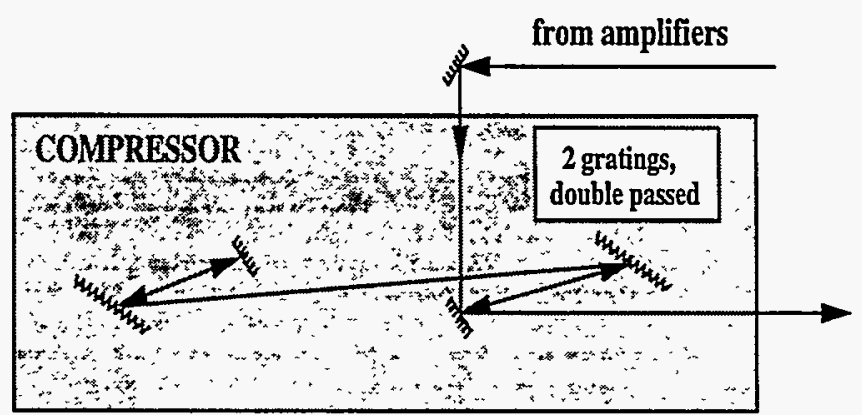

Figure 3.2: Schematic of the $10 \mathrm{TW}$ laser system 
in time by the round trip time of the cavity, 12 nanoseconds. Inside the regenerative amplifier, the pulse makes 14 passes, amplifying the initial $18 \mathrm{pJ}$ pulse up to the $4 \mathrm{~mJ}$ level, resulting in a net gain of approximately $10^{8}$. The amplifying medium in the regenerative amplifier consists of two flashlamp pumped neodymium doped (3\%) glass rods. These rods are made of the phosphate glasses HAP3 and APG1. The gain peaks for these two glasses are at $1052.5 \mathrm{~nm}$ and $1053.5 \mathrm{~nm}$, respectively. The position of the gain peaks relative to the central laser wavelength $(1052.7 \mathrm{~nm})$ provides an effective amplitude gain bandwidth greater than that exhibited by either glass alone. Gain narrowing experienced by the laser pulse in each medium is used in this system to shape the spectrum by balancing the relative gain in each amplifier. [91] This results in a higher contrast ratio than would be achieved in the absence of gain narrowing since, with no spectral shaping, the hard edges of the flat-top beam would be clipped on the amplifiers, reducing the contrast ratio between the peak of the pulse and the base. The regenerative amplifier in this system is a true $\mathrm{TEM}_{00}$ laser cavity, producing Gaussian spatial output. The temporal pulse remains Gaussian as well.

The laser pulse is relay imaged [55] to a serrated aperture, which spatially limits the edges of the gaussian pulse to produce a square or flat-top beam in the near field. The resulting $1.5 \mathrm{~mJ}$ pulse is relayed with an air spatial filter (ASF, $\mathrm{f \# =}$ 61) through two flashlamp pumped $9 \times 115 \mathrm{~mm}$ neodymium doped glass rods which serve as the first set of amplifiers. These rods are made of HAP3 Nd:phosphate glass. This amplifier produces a gain of approximately 27, amplifying the pulse up to $40 \mathrm{~mJ}$. A vacuum spatial filter (VSF1, $\mathrm{f} \#=17.4,200 \mu \mathrm{m}$ pinhole) and a permanent magnet Faraday rotator are traversed before the pulse is amplified by a $19 \times 235 \mathrm{~mm}$ rod. This APG1 laser glass rod is also flashlamp pumped. A second vacuum spatial filter (VSF2, $\mathrm{f} \#=8.7,400 \mu \mathrm{m}$ pinhole) and another Faraday isolator are encountered before the pulse reaches the third amplifier. The final amplifier is a flashlamp pumped $45 \times 265 \mathrm{~mm} \mathrm{Nd}$ :glass rod (APG1). The laser is then focussed into a third vacuum spatial filter (VSF3, $\mathrm{f} \#=20,400 \mu \mathrm{m}$ pinhole). After this final amplifier, the pulse is 
$640 \mathrm{psec}$ in duration and contains up to $8 \mathrm{~J}$. Gain narrowing in the amplifiers leads to a Gaussian-like pulseshape with a bandwidth of $3.2 \mathrm{~nm}$. The spatial profile remains

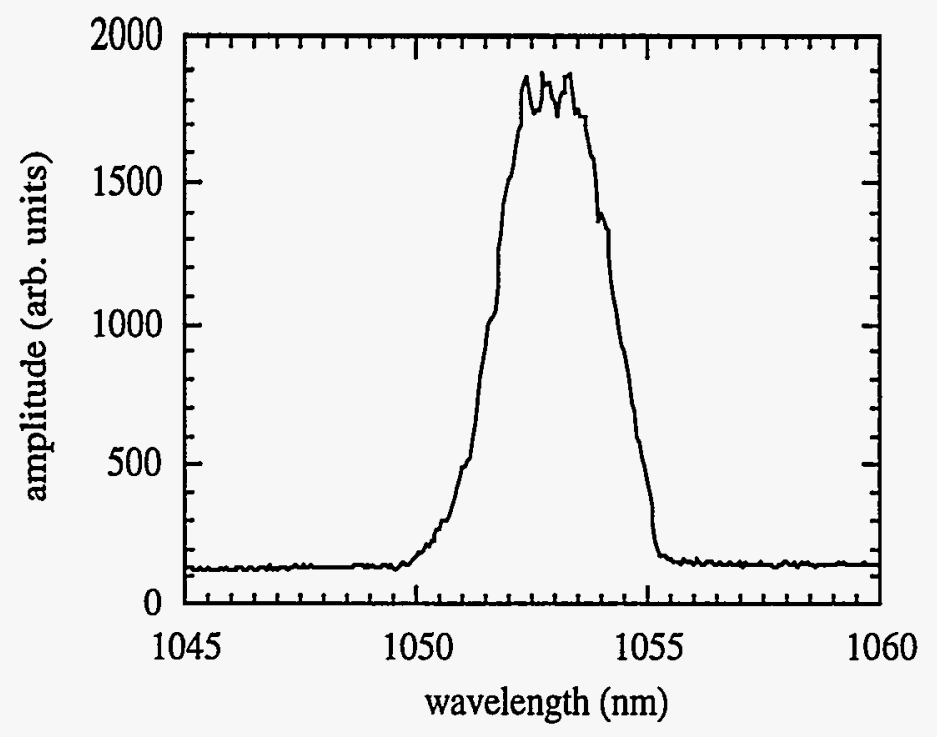

Figure 3.3: Amplified spectrum of the 10 TW laser

a flat-top due to relay imaging. The amplified stretched pulse can now be temporally recompressed to a short, intense laser pulse.

The compressor consists of a large grating pair (twelve inch diameter, 1740 grooves $/ \mathrm{mm}$ ), which are matched to the stretcher. Matching the gratings in the stretcher and compressor is important to minimize distortion effects and chirp on the compressed pulse. The minimum temporal width of the compressed pulse is limited by the bandwidth of the amplifiers. In this laser, where glass is used as the amplifying medium, the bandwidth is only $2.8 \mathrm{~nm}$, which limits the minimum pulsewidth achievable to several hundred femtoseconds. In contrast, if Ti:sapphire were used in the amplifiers, the minimum pulsewidth would be closer to $100 \mathrm{fsec}$ (bandwidth=13 nm). The gratings are double passed (see figure 3.2) and the final output of this system after the compressor is a $600 \mathrm{fsec}$ pulse containing up to $5 \mathrm{~J}$ in an $8.4 \mathrm{~cm}$ diameter beam.

Figure 3.3 shows the amplified spectrum of this laser system. The bandwidth of the amplified laser pulse is $3.2 \mathrm{~nm}$. An autocorrelation trace is shown in figure 
3.4, illustrating the Gaussian-like shape of the temporal pulse. The main laser pulse

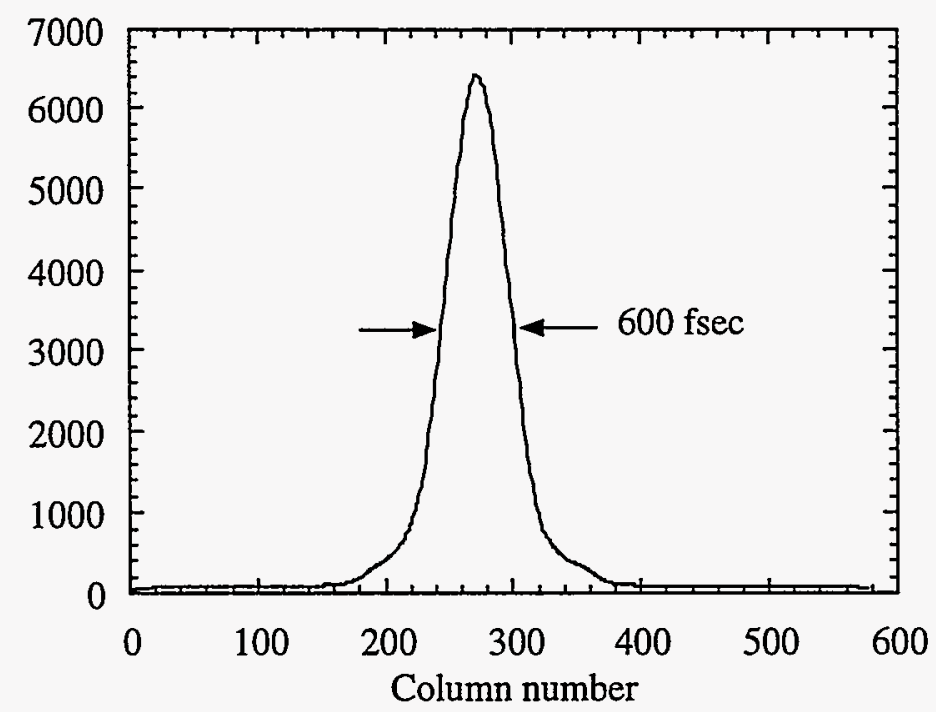

Figure 3.4: Autocorrelation trace of unamplified laser pulse

rests on a $300 \mathrm{psec}$ long pedestal which is reduced in amplitude from the peak of the pulse by approximately $10^{3}$. Since the peak intensity of the laser is near $10^{18} \mathrm{~W} / \mathrm{cm}^{2}$, this pedestal can preform a plasma several picoseconds before the peak of the laser pulse arrives in the focal region. When gas targets are used to generate the plasma, preforming a plasma with the pedestal is not a problem since this merely reduces the role of the main pulse in ionization. For solid target experiments, on the other hand, preforming a plasma several picoseconds before the main laser pulse is undesirable since it produces an expanding plasma and can hinder the desired laser-target interaction. The diffraction limited time-bandwidth product, $\Delta \nu \Delta t$, for this system is typically $0.44(+5-10 \%)$.

Since this laser system produces a flat-top in the near field, an Airy pattern is produced in the focal plane. Use of Gaussian terminology is therefore of limited use in describing the characteristics of this laser beam in the focal region. Many of the terms and formulas used in this work are Gaussian based, but since the central lobe of the Airy pattern can be approximated as a Gaussian, the results obtained with 


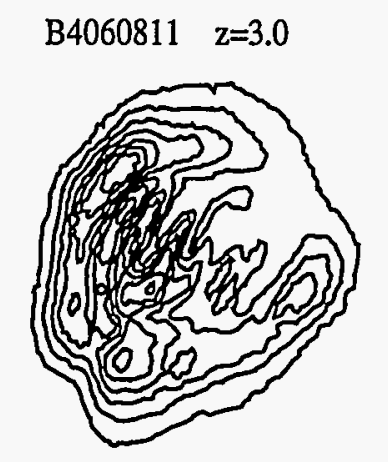

$$
\text { B4060801 } z=4.0 \quad \text { B } 4060802 \quad z=5.0 \quad \text { B } 4060803 \quad z=6.0
$$
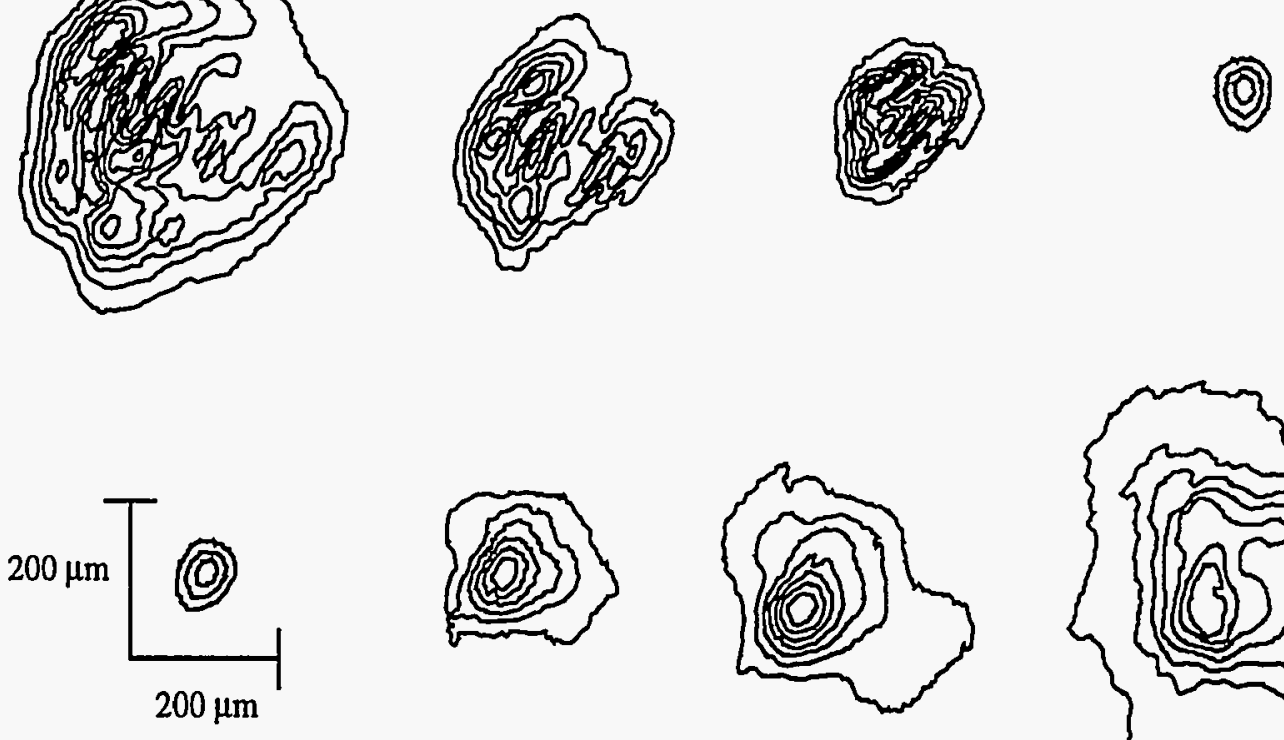

B4060804 $\quad z=7.0$

B4060805 $\mathrm{z}=8.0$
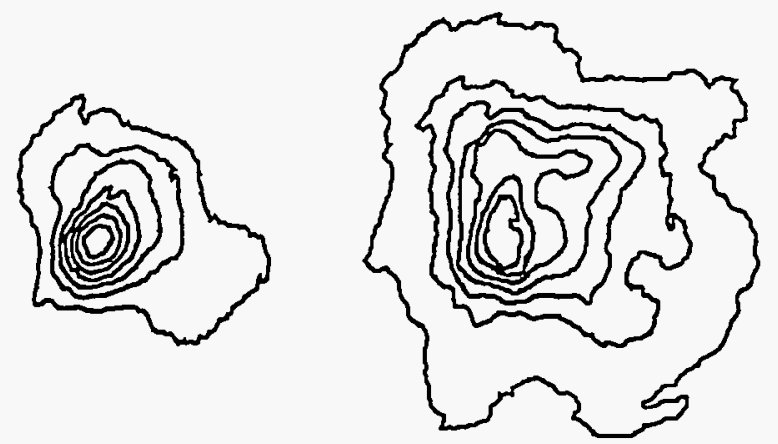

B4060806 $z=9.0$

B4060807 $\mathrm{z}=10.0$

Figure 3.5: Contour plots of the laser spot before and after best focus

these formulas are reasonable.

The focal depth of this laser, defined as the longitudinal distance between the half intensity points on each side of the focus, has been measured to be $1.2 \mathrm{~mm}$. This value was obtained by measuring the full-width at half-maximum intensity of focal spot images taken at various positions on each side of best focus. Imaging of the focal spot at high intensity reveals a slightly astigmatic focal spot with major and minor diameters of $16 \mu \mathrm{m}$ and $24 \mu \mathrm{m}$, respectively, at the full width-half maximum of the intensity. The astigmatism of the focal spot results from astigmatic wavefront quality of the gratings in the compressor. For an Airy pattern, the principal lobe should have a full-width at half-maximum intensity diameter of $2(3.27 / \pi) f \lambda$. For a $1.0527 \mu \mathrm{m}$ laser and $f=8.2$, i.e., parameters of the experiments of this work, this expression gives a FWHM spot diameter of $18 \mu \mathrm{m}$. Therefore, the measured spot is approximately 
diffraction limited (to within the error of the spot measurement). The principal lobe of the Airy pattern contains $84 \%$ of the total energy within a $2.44 f \lambda$ diameter spot. [20] Approximately $48 \%$ of the laser energy should be contained within the FWHM spot. The quantity usually referred to as the "spot size" ( $\left.w_{0}\right)$ is a Gaussian beam quantity which refers to the radius of the focal spot at the $1 / \mathrm{e}^{2}$ point of intensity. Since the central Airy lobe can be approximated by a Gaussian, an estimate can be obtained for $\mathrm{w}_{0}$ if the FWHM spot is known $\left(\mathrm{I}=\mathrm{I}_{0} \mathrm{e}^{-2 \mathrm{r}^{2} / \mathrm{w}_{0}{ }^{2}}\right)$. For a FWHM spot of $16 \mu \mathrm{m} \times 24 \mu \mathrm{m}$, the "spot size" is $13.6 \mu \mathrm{m} \times 20.4 \mu \mathrm{m}\left(\mathrm{w}_{\mathrm{x}} \times \mathrm{w}_{\mathrm{y}}\right)$. In the remainder of this work, the intensity values cited have been calculated with the standard Gaussian formalism. I.e.,

$$
\mathrm{I}_{\text {ave }}=\frac{2\left(0.84 \mathrm{E}_{\mathrm{L}}\right)}{\pi \mathrm{w}_{\mathrm{x}} \mathrm{w}_{\mathrm{y}} \tau}
$$

Contour plots of the laser spot as it propagates through best focus are shown in figure 3.5. The separation in $\mathrm{z}$ between images is $1 \mathrm{~mm}$ and each contour represents a $14 \%$ change in intensity. The best focus lies between the positions $z=6.0$ and $z=7.0$. The contour plots show the distribution of energy in the laser spot before and after the best focus. Clearly the spot has a more uniform energy distribution after best focus than before.

\subsection{Light Collection Diagnostics}

The main light diagnostics for the experiments were collection and spectral resolution of direct backscatter, transmitted, and near forward scattered light. The optical paths for these diagnostics are illustrated in figures 3.1(a), 3.1(b), and 3.1(d).

Light directly backscattered from the interaction of the laser with the plasma was recollimated by the $69 \mathrm{~cm}(\mathrm{f} / 8.2)$ focussing lens and collected as leakage through the final turning mirror (see figures 3.1(a),(d)). This radiation was down-collimated by a pair of lenses and directed to a spectrometer, where the light was focused onto the input slit, which ranged in size from 50-150 $\mu \mathrm{m}$. In the experiments to monitor backscattered SRS, i.e., figure 3.1(a), a $0.25 \mathrm{~m}$ Czerny-Turner spectrometer was used 
with a $15 \mathrm{~cm}$ focal length lens. A long-pass filter was used at the entrance to the spectrometer to reduce second order peaks from wavelengths less than $700 \mathrm{~nm}$. Neutral density filters were placed in front of the focussing lens prior to the spectrometer when attenuation was needed to reduce signal levels. Two gratings were used in these experiments to generate spectra; a 150 grooves $/ \mathrm{mm}$ grating was used for low dispersion, and a 1200 grooves/mm grating was used for high dispersion. The resolution of the spectrometer was limited to 4 Åfor low dispersion and 0.5 Åfor high dispersion. A liquid nitrogen cooled CCD camera (Princeton Instruments) was used to record the spectra. In the experiments investigating the behavior of forward SRS, i.e., figure 3.1(d), backscattered light was focussed into a $0.3 \mathrm{~m}$ Czerny-Turner spectrometer with a $10 \mathrm{~cm}$ lens. A 150 grooves $/ \mathrm{mm}$ grating was used to resolve the spectra and attenuation was provided with neutral density filters prior to the focusing lens. A 500 channel optical multi-channel analyzer (OMA) was used to record these spectra.

The transmitted (direct forward) light was collected with a $1 \mathrm{~m}$ lens at the output of the experimental chamber. The radiation was down-collimated and directed to the $0.25 \mathrm{~m}$ spectrometer (see figure 3.1(a)), where it was multiplexed onto the input slit with the $15 \mathrm{~cm}$ lens. This allowed simultaneous monitoring of the transmitted and backscattered light on the same spectrometer. Large (12" square) neutral density filters placed at the output of the experimental chamber provided attenuation of what was typically a very large laser signal. This attenuation was necessary to avoid self phase modulation that the air-path can induce on the spectrum. The light was spectrally resolved with either a 150 grooves $/ \mathrm{mm}$ or 1200 grooves/mm grating. The liquid nitrogen cooled CCD camera was used to record these spectra as well. The long pass filter was used at the entrance to the spectrometer to eliminate light of wavelength less than $700 \mathrm{~nm}$.

To reduce the level of laser light present in the spectra, near forward scattered light was collected inside the vacuum chamber with a $12 \mathrm{~cm}$ achromatic positive lens (see figure 3.1(b)). This lens collimated light emitting from the plasma over an angular region $5^{\circ}-15^{\circ}$ from the laser axis. The focal cone of the laser was $3.3^{\circ}$. The 
near-forward scattered light was directed to the $0.25 \mathrm{~m}$ spectrometer where it was spectrally resolved with the 150 grooves $/ \mathrm{mm}$ grating and recorded with the liquid nitrogen cooled CCD. Attenuation, when necessary, was provided by neutral density filters placed before the focusing optic of the spectrometer. The long pass filter was also used in these experiments at the entrance to the spectrometer to reduce the effects of second order peaks. The energy transmitted through the plasma within the original focal cone was also monitored in these experiments.

Figure 3.1(d) shows a second method used to collect the near forward scattered light. In this configuration, a $30 \mathrm{~cm}$ lens was used to collect light exiting the plasma and a $2.5 \mathrm{~cm}$ diameter block was placed in the beam to block the central region (up to $5^{\circ}$ from the laser axis) of the collimated beam. Light collected in this method $\left(5^{\circ}-7^{\circ}\right)$ was then directed to the $0.25 \mathrm{~m}$ spectrometer were it was spectrally resolved. The transmitted energy could not be monitored in this experimental configuration.

\subsection{Electron Energy Diagnostics}

Energetic electrons were measured in the experiments with two techniques: a simple $\mathrm{K}_{\alpha}$ flourescence method and a magnet electron spectrometer. In the $\mathrm{K}_{\alpha}$ flourescence technique [53], a metal target, typically a 25-50 $\mu \mathrm{m}$ thick tin (Sn) foil, 2" x 2", was placed ten centimeters from the focal region in the direct forward scatter direction on the focal axis (figure 3.1(c)). Electrons accelerated by the electron plasma wave which escaped the plasma region by overcoming space charge effects struck the Sn target, producing inner $(\mathrm{K})$ shell ionization, leading to emission characteristic of $\mathrm{K}_{\alpha} \mathrm{x}$-rays. In order to produce a $\mathrm{K}_{\alpha}$ emission, the energy of the electron must be greater than the $\mathrm{K}_{\alpha} \mathrm{x}$-ray energy, $25.3 \mathrm{keV}$ in the case of $\mathrm{Sn}$. Observation of $\mathrm{K}_{\alpha}$ emission is therefore an indicator of electrons with energies greater than the $\mathrm{K}_{\alpha}$ energy, $\mathrm{E} \geq \mathrm{E}_{\mathrm{K}_{\alpha}}$. In some experiments, a $50 \mu \mathrm{m}$ thick molybdenum (Mo) foil was bonded to the plasma side of the Sn foil and was used to discriminate against electrons of lower energies; this thickness of Mo is one 'practical range' for a $700 \mathrm{keV}$ electron. The $\mathrm{K}_{\alpha} \mathrm{x}$-rays were 
observed as photon 'hits' on a silicon-based liquid nitrogen cooled CCD camera used to take a spatial picture. When the CCD stops photons, the photons deposit energy in the 'wells' of the pixels, generating electron-hole pairs in the silicon. This energy shows up as counts on the CCD. The number of counts in a particular pixel is proportional to the number of electron-hole pairs created when the photon deposited its energy. Since, in silicon, $3.6 \mathrm{eV}$ of deposited energy is required to produce each electron hole pair, the number of counts can be related to the energy of the photon which was stopped by the CCD. Pulse-height analysis of the 'hits' on the CCD camera produces an $\mathrm{x}$-ray energy spectrum. The pulse-height analysis is essentially a histogram; i.e., an accounting of how many pixels had $\mathrm{K}_{\alpha}$ energy deposited in them. [75] For the experiments here, this analysis resolves the $\mathrm{Sn} \mathrm{K}_{\alpha}$ feature in spectrum (i.e., a peak at $25.3 \mathrm{keV}$ ). It should be noted that the pulse-height analysis (histogram) procedure works only when there is a sufficiently low flux of x-rays onto the CCD so that no pixel captures more than one photon. Typically, the flux level is adjusted through geometry $\left(1 / \mathrm{r}^{2}\right)$ or filtering for typical fluxes of 1 photon/10 pixels. The energy transmitted in the vacuum focal cone and the near forward scattered light could not be monitored when the $\mathrm{K}_{\alpha}$ emission diagnostic was being used since the metal foil physically blocked the transmitted and near-forward scattered light.

To obtain a more specific energy measurement, a $90^{\circ}$ imaging deflecting magnet ([76],[45]) was also used to measure energetic electrons, as shown in figure 3.1(d). This magnet is a symmetric sector magnet with rotated pole faces for vertical focusing. The magnet was placed seven centimeters past the focal region in the direct forward path. Accelerated electrons escaping the plasma reached the magnet and were deflected along trajectories determined by the energy of the particular electron, and the strength of the magnetic field. A $1 \mathrm{~mm}$ thick silicon surface barrier detector was used to collect the electrons (electron SBD). Varying the current applied to the magnet changed the magnetic field, and, therefore, the energy of the electrons which arrived at the detector. Figure 3.6 shows the energy of electrons deflected at $90^{\circ}$ as a function of the current applied to the magnet. The strength of the magnetic field 
between the pole faces of the magnet is also shown in figure 3.6 (right-side y-axis). For a current of $1.3 \mathrm{Amps}$, the magnetic field is $1.5 \mathrm{kG}$ and $2.0 \mathrm{MeV}$ electrons are directed toward the electron SBD. The collection angle of the detector allowed a 200 $\mathrm{keV}$ range of electrons to reach the SBD. I.e., if the magnet was tuned to allow $1 \mathrm{MeV}$ electrons to strike the detector, the actual range of energies collected would be 0.9 1.1 MeV. Since surface barrier detectors are sensitive to both $\mathrm{x}$-rays and electrons,
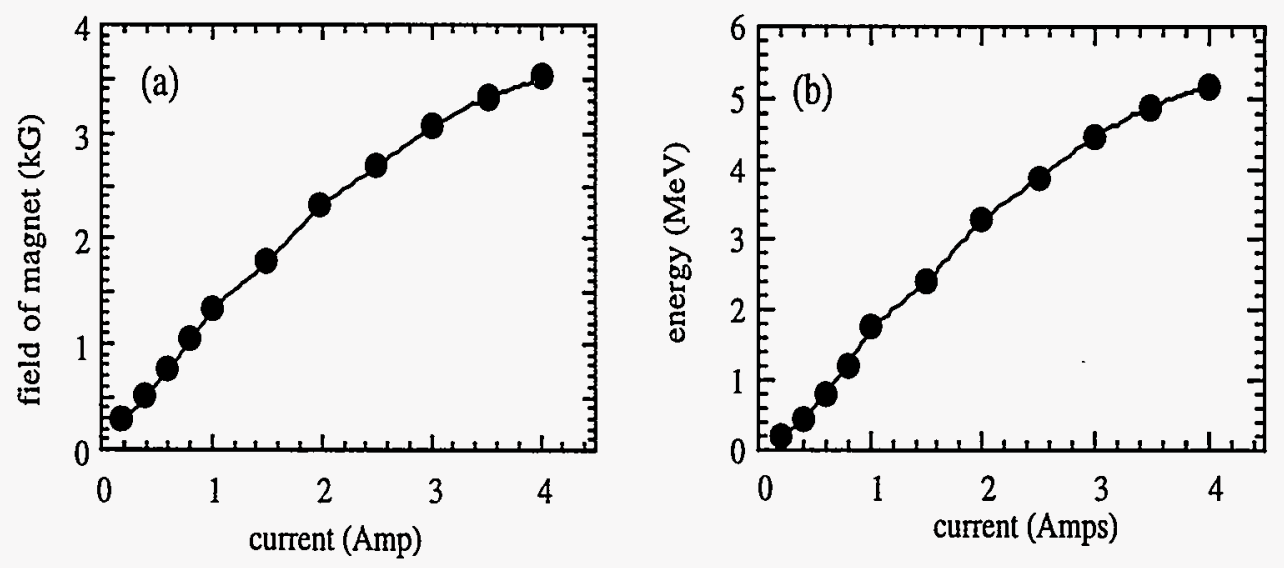

Figure 3.6: Energy of electrons reaching surface barrier detector and field of the deflecting as a function of current applied to the magnet

the electron SBD was shielded to minimize direct $\mathrm{x}$-ray flux and a second surface barrier detector (control SBD) with identical shielding was set up $135^{\circ}$ from the laser axis to monitor $\mathrm{x}$-ray emission from the plasma. The shielding on both detectors consisted of lead surrounding the detector and a $50 \mu \mathrm{m}$ copper foil in front of each SBD. Most of the hard x-rays generated in this experiment are from electrons hitting the front of the spectrometer magnet and the softer x-rays are from the plasma. The $x$-ray level on the electron SBD was measured by reversing the magnet polarity and correlating the x-ray signals measured on the two detectors. In the experiments, the electron-plus-x-ray signal was then measured on the electron SBD with the correct polarity $\mathrm{B}$ field while the control SBD once again measured the $\mathrm{x}$-ray contribution. 
This allowed for subtraction of the x-ray component of the electron SBD signal. Either near forward scattered light (as shown in figure 3.1(d)) or the energy transmitted in the vacuum focal cone could be monitored simultaneously with the electrons with this experimental configuration since the gap between the poles of the magnet did not block the laser beam. Backscattered light was also collected.

\subsection{Plasma Creation and Characterization}

\subsubsection{Creation of the Plasma}

As mentioned earlier in this chapter, a $1.0527 \mu \mathrm{m}$ short pulse laser was used to create and interact with an underdense plasma. The experiments presented in this work were perfomed with helium as the target gas, using either a static fill or a high Mach number gas jet (on loan from the UCLA Plasma Accelerator Group). Ionization induced refraction limits the plasma density achievable with a static fill. ([6], [81],[95],[97]) The maximum electron density (refraction limited) is given by [73]

$$
\left(\frac{\mathrm{n}_{0}}{\mathrm{n}_{\mathrm{cr}}}\right)_{\max } \leq \frac{\lambda}{2 \mathrm{z}_{\mathrm{R}}}
$$

where $\mathrm{n}_{\mathrm{cr}}$ is the critical density $\left(1.01 \times 10^{21} \mathrm{~cm}^{-3}\right.$ for a $1.0527 \mu \mathrm{m}$ laser $)$ and $\mathrm{z}_{\mathrm{R}}$ is the Rayleigh length. For these experiments, this limits the electron density of a static fill to $\mathrm{n}_{0}$, is limited to $2 \times 10^{18} \mathrm{~cm}^{-3}$ (assuming $\mathrm{z}_{\mathrm{R}}=0.6 \mathrm{~mm}=$ one-half the measured focal depth). Therefore, a localized burst of gas from a supersonic gas jet is utilized for experiments requiring $\mathrm{n}_{0}>2 \times 10^{18} \mathrm{~cm}^{-3}$. The General Valve gas jet, equipped with a ceramic nozzle designed to create a high Mach number burst, produces a dense, localized region of gas which is roughly cylindrical and has diameter several times the vacuum spot diameter, $\mathrm{D}_{\mathrm{vol}} \approx 100 \mu \mathrm{m}$ and length approximately the focal depth of the laser, $\mathrm{L}_{\mathrm{vol}} \approx 1 \mathrm{~mm}$. When a static fill of helium was used, the size of the plasma was determined by the region in which the laser was above the ionization threshold. Therefore, the plasmas created with static fill were quite large $\left(\mathrm{L}_{\mathrm{vol}} \leq 1 \mathrm{~cm}, \mathrm{D}_{\mathrm{vol}} \approx\right.$ $1 \mathrm{~mm})$. 
The plasma is created via tunneling ionization when the leading edge of the laser pulse enters the region of helium gas. There are two types of ionization which characterize two regimes of laser-plasma interactions: multiphoton ionization and tunneling ionization. Typically, these regimes are defined by the Keldysh parameter, $\kappa=\left(\mathrm{E}_{\text {ion }} / 2 \Phi_{\mathrm{p}}\right)^{1 / 2}$, where $\mathrm{E}_{\mathrm{ion}}$ is the ionization potential and $\Phi_{\mathrm{p}}$ is the ponderomotive potential of the laser field. [60] The tunneling regime corresponds to $\kappa<1$ while $\kappa>1$ defines the multiphoton regime. The tunneling regime can be viewed as an ionization process in which the Coulomb barrier of the nucleus is suppressed by the laser field, thereby allowing an electron to tunnel through the barrier. In contrast, multiphoton ionization occurs when an atom absorbs several photons, resulting in the liberation of an electron. For short-pulse, high intensity lasers, the laser field is typically on the order of or larger than the atomic field binding the electrons to the nucleus, allowing tunneling ionization to occur. The threshold laser intensity for tunnel ionization of a particular charge state can be estimated using [5]

$$
\mathrm{I}_{\mathrm{th}}\left(\mathrm{W} / \mathrm{cm}^{2}\right)=4.0 \times 10^{9} \frac{\mathrm{E}_{\text {ion }}{ }^{4}(\mathrm{eV})}{\mathrm{Z}^{2}}
$$

where $\mathrm{Z}$ is the ionic charge. For helium, the gas used in the experiments presented here, both electrons are removed from each atom at an intensity achieved early in the laser pulse ( 2 psec before the peak intensity for a $1 \mathrm{psec}, 10^{18} \mathrm{~W} / \mathrm{cm}^{2}$ gaussian laser pulse). In fact, experiments performed with the laser system used in this work show that the helium atoms are fully stripped with a laser intensity of $4 \times 10^{15} \mathrm{~W} / \mathrm{cm}^{2} .[32]$ This implies that, for the high intensities actually used in the experiments, a uniform plasma density is seen by the high intensity portion of laser pulse in the interaction region since the ionization occurs early at the front edge of the pulse and the plasma density is simply twice the atom density. The size and shape of plasmas produced from a static fill are discussed in more detail in appendix A. 


\subsubsection{Characterization of the Plasma}

The plasmas created from both static fill and gas jet bursts of helium were characterized with low intensity stimulated Raman backscatter. ([28],[90]) As described in chapter 2, stimulated Raman scatter is a multi-wave process in which an incident light wave decays into scattered light waves and an electron plasma wave. The frequency of the scattered light provides a measurement of the electron density since it is shifted from the laser frequency, $\omega_{0}$, by the frequency of the electron plasma wave, $\omega_{\mathrm{e}}$ as illustrated in equation (2.1). For backscattered SRS, the wavenumber of the electron plasma wave, $\mathrm{k}_{\mathrm{e}}$, is approximately $2 \mathrm{k}_{0}$, which allows equation (2.5) to be rewritten as

$$
\omega_{\mathrm{e}}=\omega_{\mathrm{pe}} \sqrt{1+12 \frac{\mathrm{v}_{\mathrm{th}}{ }^{2} \omega_{0}^{2}}{\mathrm{c}^{2} \omega_{\mathrm{pe}}{ }^{2}}}
$$

The frequency shift has a weak dependence on the electron temperature, $\mathrm{T}_{\mathrm{e}}$, through the thermal velocity, $\mathrm{v}_{\mathrm{th}}$, allowing for estimates of the plasma temperature as well as the density.

A typical low intensity stimulated Raman backscatter spectrum is shown in figure 3.7. The laser intensities used for the characterization are low in order to minimize the intensity dependent spectral anomalies associated with the strongly coupled regime of SRS.[34] A distinct peak is apparent on the red side of the laser wavelength, shifted by $\Delta \lambda$, which can be used to extract a frequency shift, $\Delta \omega$. The electron density can be estimated based on this shift. I.e.,

$$
\begin{aligned}
\frac{\Delta \lambda}{\lambda_{0}} & =\frac{\Delta \omega}{\omega_{\mathrm{s}}}=\frac{\omega_{\mathrm{e}}}{\omega_{0}-\omega_{\mathrm{e}}} \\
& =\frac{\sqrt{\mathrm{n} / \mathrm{n}_{\mathrm{cr}}}}{1-\sqrt{\mathrm{n} / \mathrm{n}_{\mathrm{cr}}}}
\end{aligned}
$$

where $\lambda_{0}=1.0527 \mu \mathrm{m}$, the wavelength of the laser. This expression can be rewritten as

$$
\mathrm{n}=\mathrm{n}_{\mathrm{cr}}\left(\frac{\Delta \lambda}{\lambda+\Delta \lambda}\right)^{2}
$$




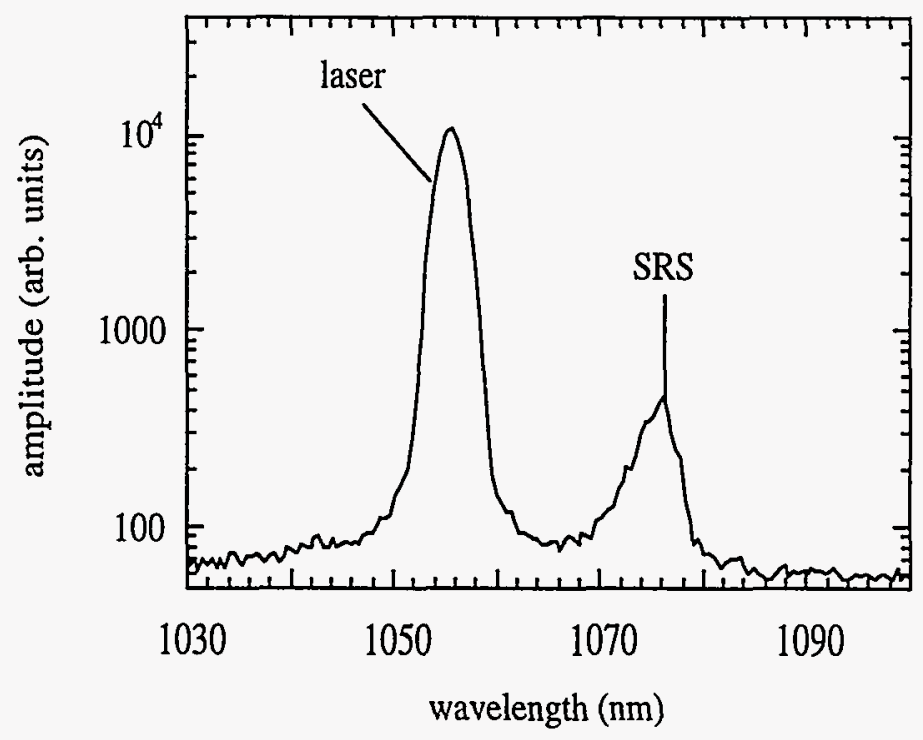

Figure 3.7: Low intensity SRS spectrum obtained with a helium static fill pressure of 5.4 torr

The spectrum shown in figure 3.7 was obtained with a static fill helium pressure of 5.4 torr and a laser energy of $0.25 \mathrm{~J}$. The electron density inferred from $\Delta \omega$ is $4.55 \times 10^{17} \mathrm{~cm}^{-3}$ if the electron temperature contribution is negligible. For static fill SRS spectra, an estimate of the plasma density can also be obtained using the known pressure of gas in the chamber. The electron density of the fully stripped helium plasma in the interaction region is simply twice the atom density and can be found from the ideal gas law

$$
\mathrm{p}=\mathrm{nkT}
$$

which becomes, upon rearranging terms and assuming that $\mathrm{T}=293^{\circ} \mathrm{K}$,

$$
\mathrm{n}\left(\text { in } \mathrm{cm}^{-3}\right)=3.28 \times 10^{16} \mathrm{pZ}
$$

where $\mathrm{p}$ is the static fill pressure (in torr) and $\mathrm{Z}$ is the ionization state ( $\mathrm{Z}=2$ for helium). For the static fill pressure of figure 3.7, the calculated plasma density should be $3.54 \times 10^{17} \mathrm{~cm}^{-3}$. By comparing electron density measurements obtained from the backscattered SRS, $n_{\text {srs }}$, and from the static fill pressure, $n_{s f}$, the temperature of the 
plasma can be estimated through the thermal correction term of equation (3.4).

$$
\mathrm{T}_{\mathrm{e}}=\frac{511000}{12}\left[\frac{\mathrm{n}_{\mathrm{srs}}-\mathrm{n}_{\mathrm{sf}}}{\mathrm{n}_{\mathrm{cr}}}\right]
$$

For the spectrum shown in figure 3.7, an electron temperature of $\sim 5 \mathrm{eV}$ is found. Figure 3.8(a) illustrates the dependence of the wavelength shift of the SRS feature on the static fill pressure. These shifts were obtained at low laser intensity $(\leq 1 \times$ $10^{17} \mathrm{~W} / \mathrm{cm}^{2}$ ). As the static fill pressure of helium was increased, the shift between the laser wavelength and the SRS feature increased. Figure 3.8(b) shows the relationship between the known density of the static fill and the inferred density from the SRS (assuming $\mathrm{T}_{\mathrm{e}}=0 \mathrm{eV}$ ). If the temperature of the electrons was exactly equal to zero, the density inferred from the SRS shift should be exactly the known static fill density
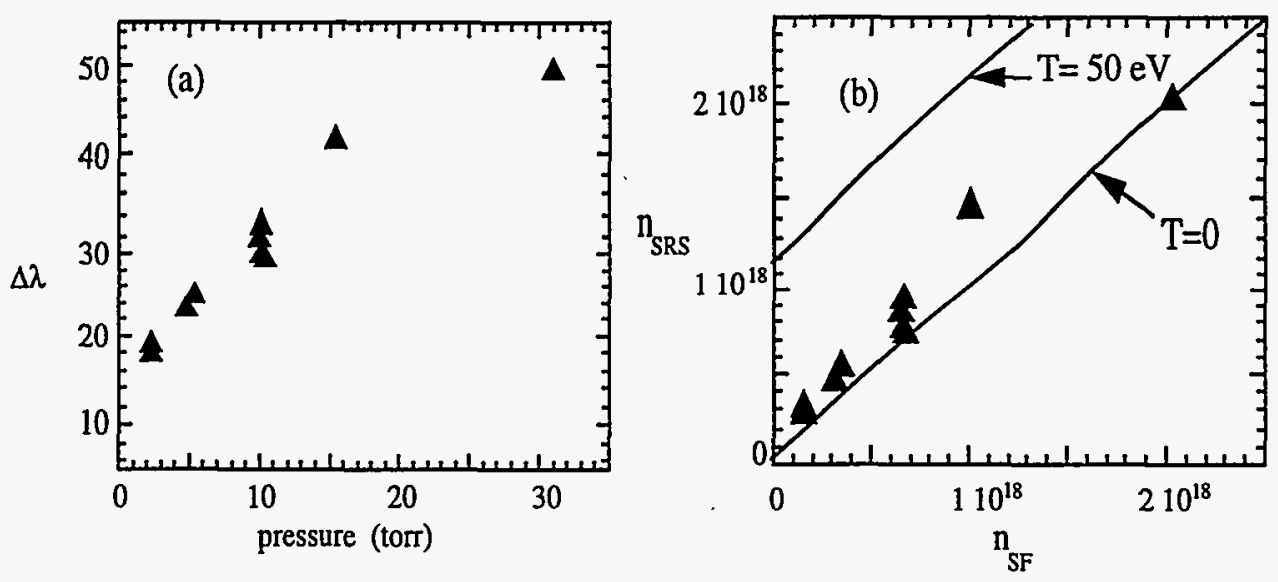

Figure 3.8: (a) Wavelength shift of SRS feature as a function of helium static fill pressure. (b) Density inferred from SRS shift versus density of known static fill.

(depicted by $\mathrm{T}=0$ line). If the electron temperature was $50 \mathrm{eV}$, the density inferred from the SRS shift would lie along the line $\mathrm{T}=50 \mathrm{eV}$. Clearly, for SRS obtained with low laser intensity, the electron temperature values inferred from the SRS spectra at a variety of static fill pressures are all less than $50 \mathrm{eV}$.

Since the static fill experiments indicate that the thermal correction of equation 
(3.4) is small, it is neglected in the gas jet density measurements and the electron density is inferred by simply assuming that the frequency shift, $\Delta \omega$, is equal to the plasma frequency, $\omega_{\mathrm{pe}}$. Figure 3.9 shows the variation of the electron density of the helium plasma with the backing pressure applied to the gas jet. There is typically some shot-to-shot fluctuation in the inferred density, but this can be partly attributed to irreproducibility of the gas jet. In general, however, the electron density inferred from the frequency shift increased with increasing backing pressure, as expected.

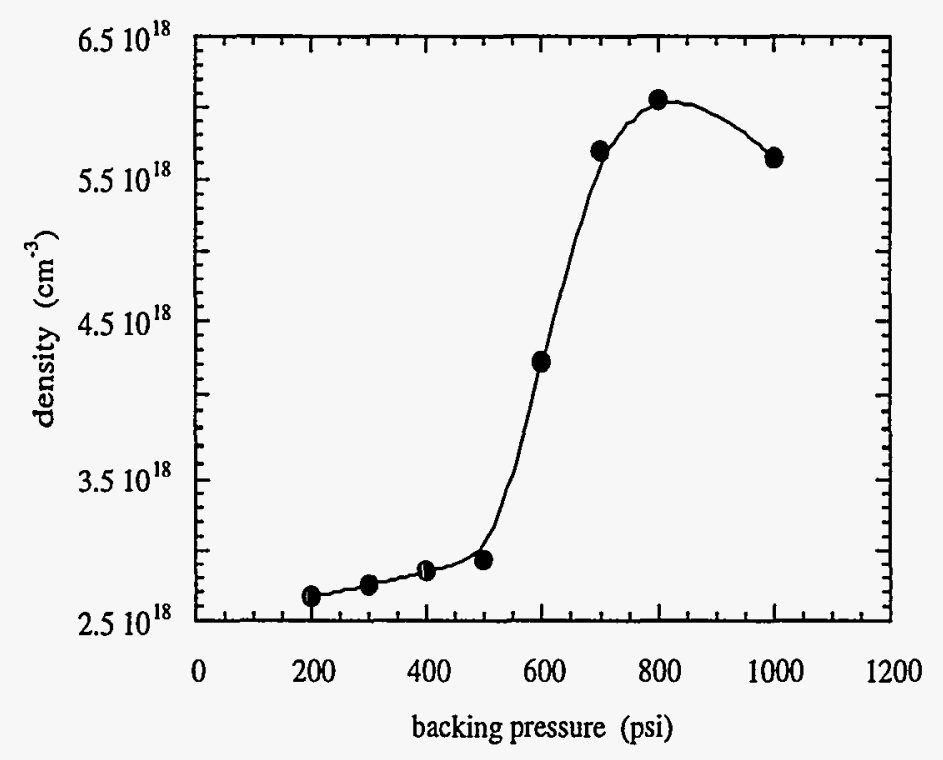

Figure 3.9: Variation of electron density with backing pressure of the gas jet 


\section{Chapter 4}

\section{Forward Stimulated Raman}

\section{Scatter}

The results of experiments studying forward stimulated Raman scattering in short pulse, high intensity laser underdense plasma interactions are presented in this chapter. The parameter regime in which forward SRS occurs is described and observations of the signatures of forward SRS (spectra and energetic electrons) are presented. Results of experiments studying how forward SRS affects the propagation of the laser pulse and the implications of these observations are also presented.

\subsection{Parameter Regime}

For forward SRS to be observable in laser-plasma interaction experiments, appreciable e-foldings of the instability must occur. In particular, $\gamma_{0} \tau$, which represents the growth of the instability during the time the laser propagates through the plasma, where $\gamma_{0}$ is the relativistic growth rate for forward SRS (equation (2.97)) and $\tau$ is the propagation time, must be $\geq 8$. This corresonds to a total gain of $e^{\gamma_{0} \tau} \geq 3000$. The parameter regime in which forward SRS can be studied is defined by this condition, as shown in figure 4.1. Values relevant for the laser system described in chapter 3, $\omega_{0}=1.79 \times 10^{15} \mathrm{sec}^{-1}$ and $\tau=3600$ fsec ( 6 times the laser pulsewidth) were used 
to obtain this plot. The shaded area of figure 4.1 shows the densities and intensities for which the forward Raman instability should be observable based on $\gamma_{0} \tau \geq 8$. The

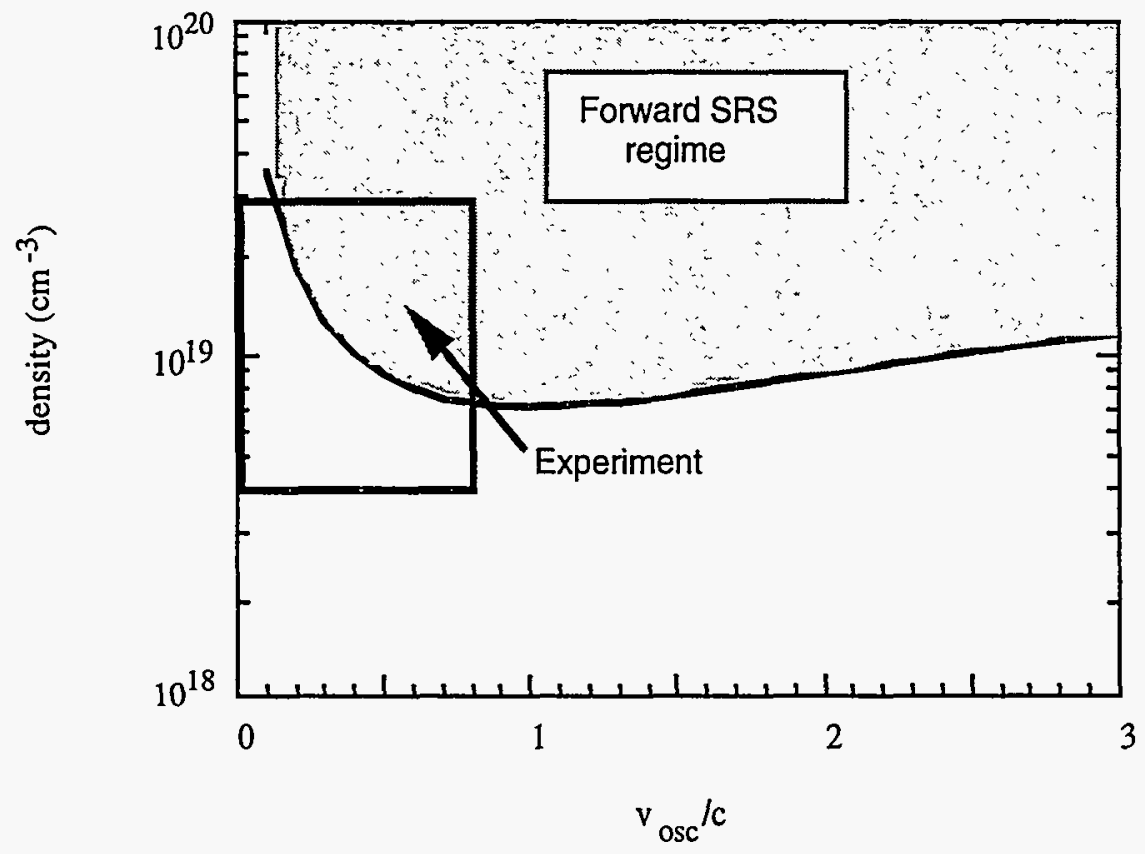

Figure 4.1: Regime in which forward SRS can be observed

experimentally accessible regime, defined by the intensity available from the laser and the density obtainable with the gas jet, is also shown. The overlap region of figure 4.1 specifies the parameter regime in which the experiments to study forward Raman scattering were performed in this work. Specifically, the laser intensities used in the experiments ranged from $\mathrm{v}_{\mathrm{osc}} / \mathrm{c}=0.2$ to $\mathrm{v}_{\mathrm{osc}} / \mathrm{c}=0.8$ and the plasma density was restricted to $8 \times 10^{18} \mathrm{~cm}^{-3} \leq \mathrm{n}_{0} \leq 3 \times 10^{19} \mathrm{~cm}^{-3}$. For these parameters, the number of e-foldings of the forward SRS instability ranges from $\gamma_{0} \tau=3.7$ to $\gamma_{0} \tau=36$. The total gain (for unchecked growth), $\mathrm{e}^{\gamma_{0} \tau}$, ranges from 38.5 to $4 \times 10^{15}$. Clearly, a gain of $10^{15}$ is not physical, but this calculation does not include nonlinear saturation effects which will limit the actual growth in experiments. It is worth noting that the level of growth necessary for experimental observation of forward SRS can be estimated from the gain. For $\gamma_{0} \tau=17, \mathrm{G}=\mathrm{e}^{\gamma_{0} \tau}=2.4 \times 10^{7}$. The amplitude of the scattered 
light wave would then be the product of the gain times the noise intensity, $G * I_{\text {noise }}$. Assuming a typical Thomson scatter noise level of $10^{-10}, \mathrm{G} * 10^{-10}=2.4 \times 10^{-3}$, which should be observable experimentally. In fact, the anti-Stokes features observed (discussed in section 4.2) are typically $10^{3}-10^{4}$ above the noise floor of the detection system. The plasmas studied in the experiments were created from a burst of gas from a gas jet (see section 3.5.1). This limits the size of the plasma to approximately $1 \mathrm{~mm}$, which is approximately the focal depth for this laser.

\subsection{Forward Stimulated Raman Scatter Spectra}

The spectral signature of forward stimulated Raman scatter is the presence of Stokes and anti-Stokes modes, as seen in section 2.3 where the dispersion relation for SRS was solved. This solution showed spectral features shifted from the laser frequency, $\omega_{0}$, by approximately $-\omega_{\mathrm{pe}}$ (Stokes) and $+\omega_{\mathrm{pe}}$ (anti-Stokes). In fact, a cascade of several Stokes and anti-Stokes modes is expected. ([37],[48]) The spectral cascade does not appear in the dispersion relation solution because only first order effects were considered (i.e., $\omega_{0} \pm \omega_{\mathrm{pe}}$ ). If higher order coupling terms are retained in the dispersion relation (equation (2.65), i.e., $\omega_{0} \pm \mathrm{m} \omega_{\mathrm{pe}}, \mathrm{m} \geq 1$ ), higher order Stokes and anti-Stokes features are obtained when it is solved.

The frequency matching condition, equation (2.1), reflects the spectral cascading and is given by

$$
\omega_{\mathrm{s}}=\omega_{0} \pm \mathrm{m} \omega_{\mathrm{pe}}
$$

where $\mathrm{m} \geq 1$ and the $+(-)$ refers to the anti-Stokes (Stokes) feature. It is clear from equation (4.1) that adjacent Stokes or anti-Stokes peaks have a constant frequency separation, $\omega_{\text {pe }}$. This separation is independent of the order of the feature. The wavelength separation, $\Delta \lambda_{\mathrm{m}}$, however, is not constant, and is given by:

$$
\begin{aligned}
\Delta \lambda_{\mathrm{m}} & =\left(\lambda_{\mathrm{m}}-\lambda_{\mathrm{m}-1}\right) \\
& =\lambda \sqrt{\frac{\mathrm{n}_{0}}{\mathrm{n}_{\mathrm{cr}}}}\left[\left(1 \mp \mathrm{m} \sqrt{\frac{\mathrm{n}_{0}}{\mathrm{n}_{\mathrm{cr}}}}\right)\left(1 \mp(\mathrm{m}-1) \sqrt{\frac{\mathrm{n}_{0}}{\mathrm{n}_{\mathrm{cr}}}}\right)\right]^{-1}
\end{aligned}
$$


where $\lambda_{0}$ is the laser wavelength, $\mathrm{m}$ is the order of the peak, and the minus (plus) sign refers to the Stokes (anti-Stokes) mode. This formula can be used to predict where (in $\lambda$-space relative to the laser fiducial) the spectral signature of forward SRS should appear for a particular plasma density.

In the first set of experiments designed to detect forward Raman scatter, light propagating along the axis of the laser within the vacuum focal cone was collected and spectrally resolved. With this configuration, no SRS features were observed, most likely because the level of the scattered light wave of SRS was low relative to the level of laser light propagating in the forward cone. In order to minimize the laser light contribution to the spectra, near-forward scattered light was collected and spectrally resolved to look for the forward SRS features. This experimental configuration is illustrated in figure 3.1 and described in section 3.3. The laser light should be contained within a $3.3^{\circ}$ focal cone based on the f-number of the focusing lens. In the near-forward collection system, light is collected over $5^{\circ}-15^{\circ}$.

Figure 4.2 shows an experimental spectrum obtained for the near-forward scattered light (solid line). The first and second anti-Stokes modes were observed, as well as scattered laser light. This spectrum was obtained with a helium backing pressure of 800 psi and a laser energy of approximately $3 \mathrm{~J}$. The Stokes modes were not observed in any of the forward SRS experiments, but the CCD camera used to record the spectra is silicon based, and therefore not sensitive to wavelengths above $1100 \mathrm{~nm}$, which is where the Stokes features would appear. This poor sensitivity precluded the observation of the Stokes features. The frequency separation between the first and second anti-Stokes modes and the laser light and the first anti-Stokes feature were $102 \mathrm{~nm}$ and $138 \mathrm{~nm}$, respectively. Equation (4.2) predicts, for a plasma density of $2.25 \times 10^{19} \mathrm{~cm}^{-3}$, a $137 \mathrm{~nm}\left(\Delta \lambda_{1}\right)$ separation between the laser and the first anti-Stokes and a $105 \mathrm{~nm}\left(\Delta \lambda_{2}\right)$ separation between the first and second anti-Stokes modes. The predicted values are in excellent agreement with those observed in the spectra. The dashed line of figure 4.2 shows the first Stokes and anti-Stokes features predicted by the dispersion relation for $n_{0}=2.3 \times 10^{19} \mathrm{~cm}^{-3}$ and $\mathrm{v}_{\text {osc }} / \mathrm{c}=0.8$. The 


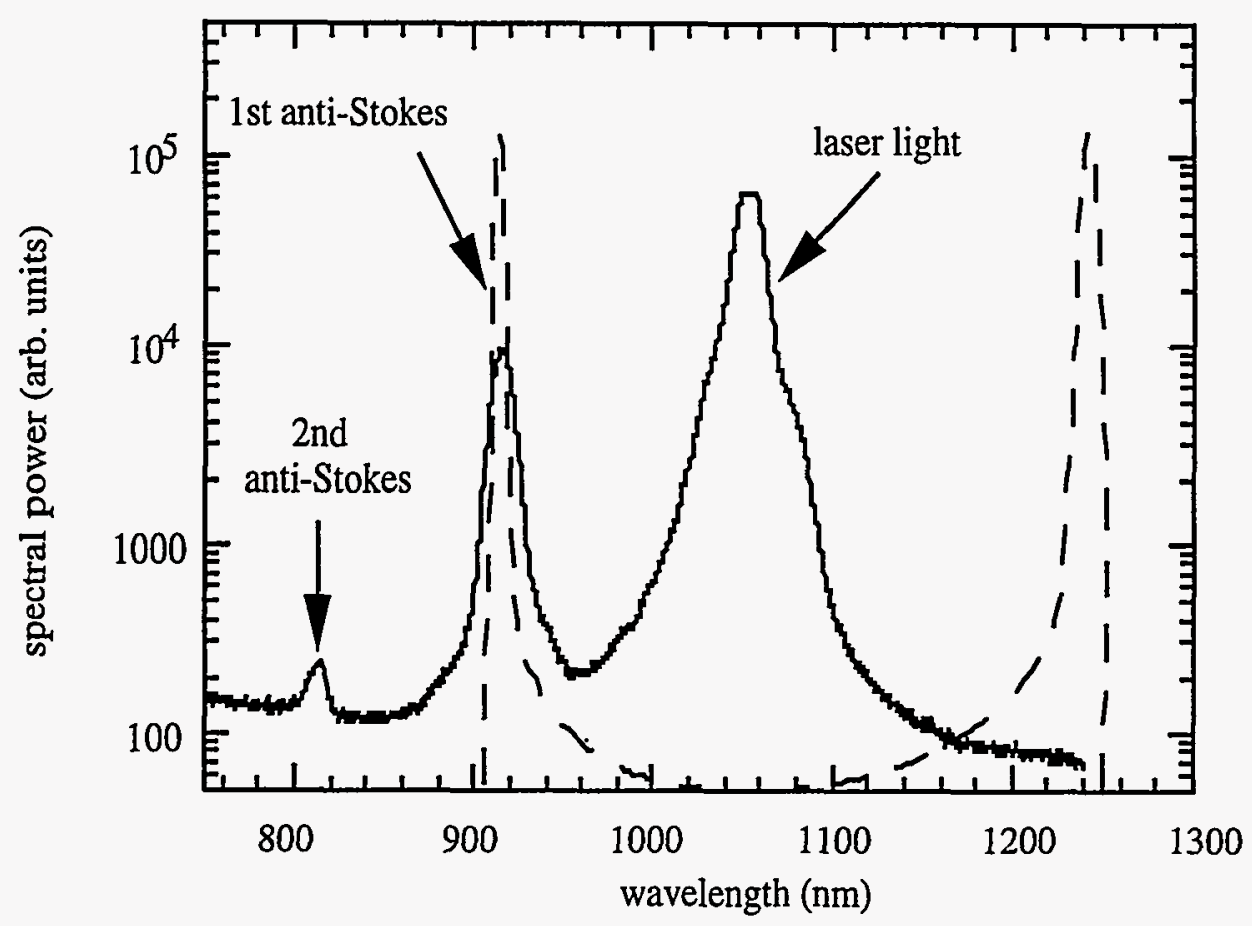

Figure 4.2: Spectrum of near-forward scattered light showing scattered laser light, and the first and second anti-Stokes features of forward stimulated Raman scattering. The dashed line shows the result predicted from the dispersion relation (retaining only first Stokes and anti-Stokes).

location of the calculated spectrum agrees with the observed spectrum. The second anti-Stokes feature of the experimental spectrum is not predicted by the dispersion relation calculation because the higher order terms were neglected in the calculation.

The dynamic range of the detector $(10,000: 1)$ limits the number of anti-Stokes modes observable since the amplitudes of the modes fall off with increasing order. The ratio second anti-Stokes/first anti-Stokes for the spectrum of figure 4.2 is 0.027 . Since the amplitudes of the anti-Stokes features decrease with increasing order, higher order anti-Stokes features would not be observable above the noise level. For example, if the ratio of the third anti-Stokes/second anti-Stokes was also 0.027 (a high estimate of the amplitude ratio), the amplitude of the third anti-Stokes mode would be substantially below the noise level of the detector. The second anti-Stokes feature was not observed on every shot and there was substantial shot-to-shot variation in the amplitude of the 
first anti-Stokes mode. Some of this fluctuation is likely due to irreproducibility of the gas jet burst and timing fluctuations between the laser and the gas jet.

The density inferred from the location of the first anti-Stokes feature (obtainable from equation (4.2)) is shown in figure 4.3(a) (open circles). The stimulated Raman backscatter was simultaneously monitored on many shots and the plasma density inferred from that frequency shift is shown in figure 4.3(a) (solid diamonds). There is substantial fluctuation on a shot-to-shot basis in the plasma density inferred from both the forward and backscattered SRS (20-30\%). Increasing the backing pressure of the gas jet generally increases the plasma density, as shown in figure 3.9 in section 3.5.2 and in figure 4.3(a). The plasma density values obtained from the backscattered SRS are consistent with those extracted from the first anti-Stokes feature, except at the lower backing pressures, where the density inferred from the backscattered is larger than that inferred from the forward SRS. The general agreement between the backscatter and forward scatter densities further strengthens the argument that the features in the near-forward spectra are indeed forward SRS modes. The frequency shift of the first anti-Stokes mode increased with increasing density, as shown in figure 4.3(b). The solid line shows the expected frequency shift as a function of plasma density for the first anti-Stokes mode. The experimental frequency shifts and predicted shifts are in excellent agreement.

The amplitude of the first anti-Stokes mode of the forward SRS varied with both incident laser intensity and plasma density, as illustrated in figure 4.4. At low laser intensities $\left(\mathrm{v}_{\mathrm{osc}} / \mathrm{c} \leq 0.4, \mathrm{P} / \mathrm{P}_{\mathrm{cr}} \leq 0.6\right.$ ), where there were few e-foldings of growth $\left(\gamma_{0} \tau \leq 12\right)$, the anti-Stokes features disappeared, as shown in figure $4.4(\mathrm{a}) . \mathrm{P}_{\mathrm{cr}}$ is defined as the critical power necessary for relativistic self focusing and is given by

$$
\mathrm{P}_{\mathrm{cr}}=17 \mathrm{GW} \frac{\mathrm{n}_{\mathrm{cr}}}{\mathrm{n}_{0}}
$$

The values in figure 4.4(a) were obtained with a fixed helium gas jet backing pressure of 800 psi. The amplitude of the first anti-Stokes mode generally increased as the intensity increased. For high laser intensities ( $\mathrm{v}_{\mathrm{osc}} / \mathrm{c} \geq 0.7, \mathrm{P} / \mathrm{P}_{\mathrm{cr}} \geq 2$ ), however, the anti-Stokes level began to decrease slightly. An increase in the amount of 

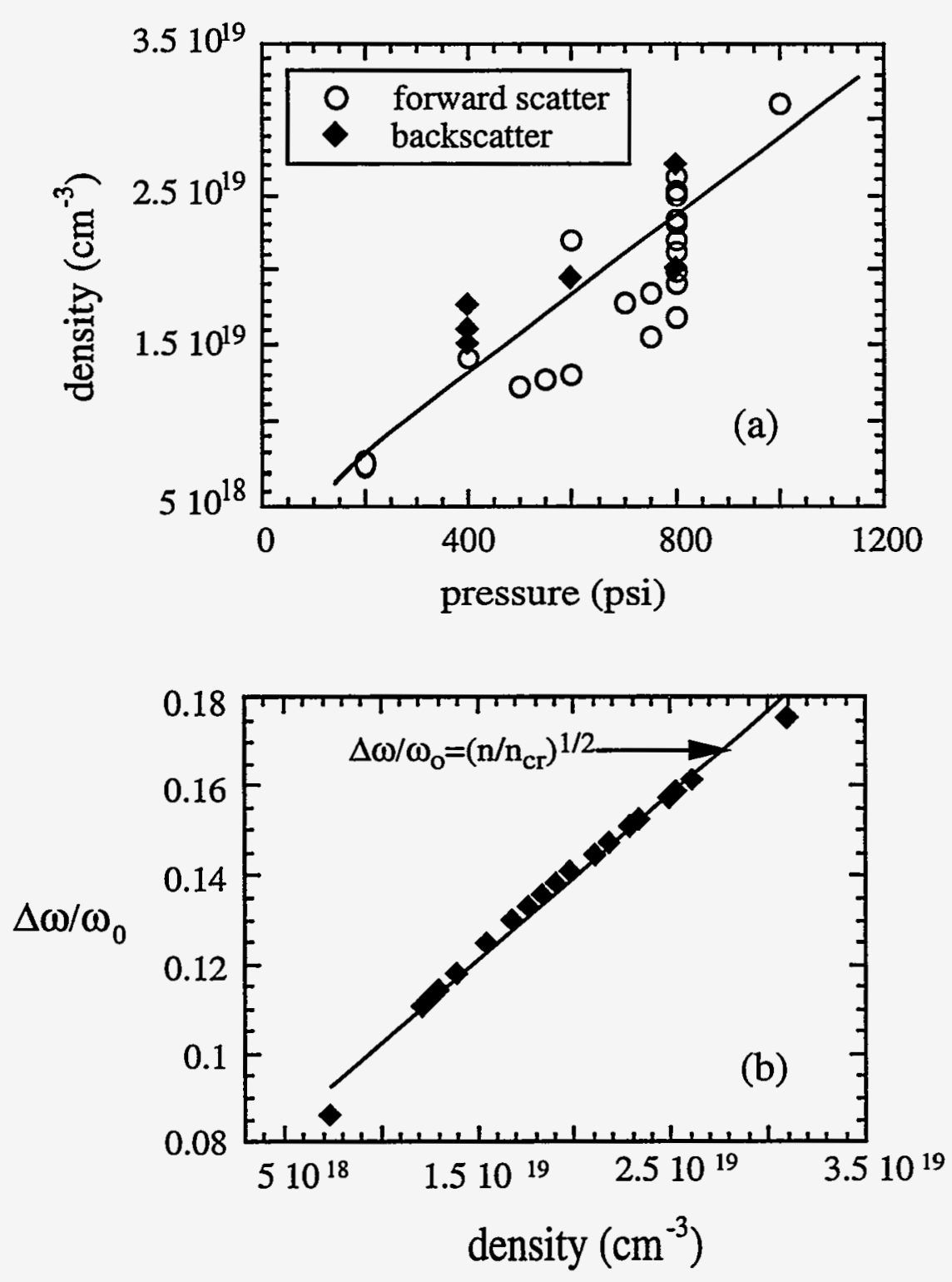

Figure 4.3: (a) The density inferred from the first anti-Stokes mode (open circles) and the backscattered SRS signal (solid diamonds) and (b) the frequency shift of the first anti-Stokes mode as a function of plasma density. 

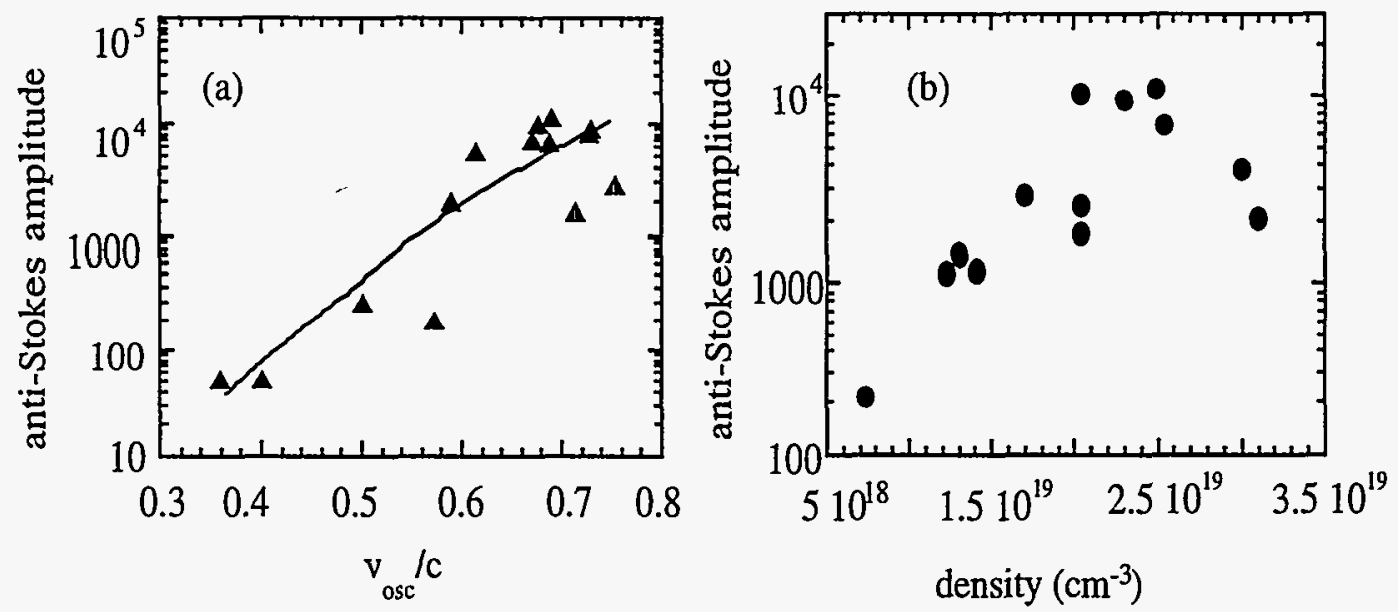

Figure 4.4: Amplitude of the first anti-Stokes feature as a function of (a) incident laser intensity (fixed backing pressure, 800 psi helium) and (b) plasma density (fixed incident intensity, $\left.\mathrm{v}_{\mathrm{osc}} / \mathrm{c} \sim 0.6\right)$.

light at the pump frequency $( \pm 10 \mathrm{~nm})$ was also observed for these intensities. At high intensity, the laser pulse contains enough power for appreciable relativistic selffocusing to occur.([114], [78], [18]) Diffraction resulting from this self focusing could limit the interaction length by reducing the f-number of the focal cone. Therefore, since the growth of forward SRS is limited by the interaction region (see chapter 1), the total growth of forward SRS will be reduced. [85] This diffraction could scatter a substantial amount of laser light outside the original focal cone of the laser, thereby explaining the large laser signals collected at $5^{\circ}-15^{\circ}$. For example, if the f-number were reduced to $\mathrm{f} / 5$, laser light would be scattered within a $5.7^{\circ}$ focal cone. The largest anti-Stokes signals were observed for $\mathrm{v}_{\mathrm{osc}} / \mathrm{c} \approx 0.67\left(\mathrm{P} / \mathrm{P}_{\mathrm{cr}} \approx 1.6\right)$.

The amplitude of the anti-Stokes feature also fell off for both low and high plasma densities, as seen in figure 4.4(b). The incident laser intensity was fixed at $\mathrm{v}_{\text {osc }} / \mathrm{c} \approx 0.6$ in this plot. For densities below $1 \times 10^{19} \mathrm{~cm}^{-3}$, the growth rate for forward SRS is small and the instability does not grow appreciably $\left(\gamma_{0} \tau \leq 12\right)$. At the higher densities (above $2.5 \times 10^{19} \mathrm{~cm}^{-3}$ ), the level of scattered light near the laser frequency increased substantially, as shown in figure 4.5. This could be due to a combination of ionization 
induced refraction [127] and diffraction resulting from relativistic self-focusing. In

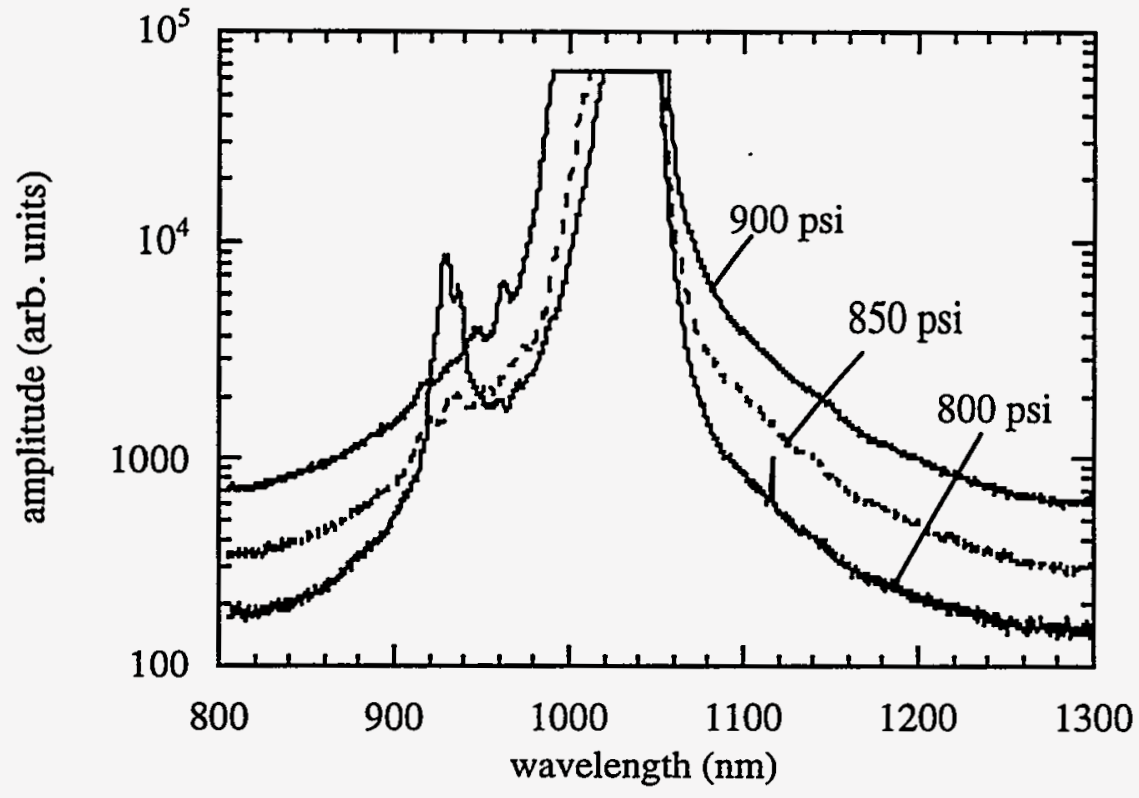

Figure 4.5: Forward SRS spectra obtained with increasing density. The laser intensity . was fixed at $\mathrm{v}_{\text {osc }} / \mathrm{c}=0.8$.

other words, it is stipulated that the anti-Stokes signal is reduced because the focusing properties of the laser are adversely affected. The increase in the scattered pump light typically occurred above $2.5 \times 10^{19} \mathrm{~cm}^{-3}$, whereas the best anti-Stokes signals were observed for densities near $2 \times 10^{19} \mathrm{~cm}^{-3}$. The slope of the anti-Stokes amplitude versus the density is much less steep than predicted by forward SRS theory (i.e., $\gamma_{0} \tau$ ). This strongly supports electron data shown later in this chapter which indicates that the electron plasma wave is driven to a very large amplitude and that nonlinear saturation. (i.e., particle trapping, pump depletion) has set in.

\subsection{Energetic Electron Measurements}

Another characteristic of forward SRS is the generation of the energetic electrons accelerated by the plasma wave, as discussed in section 2.1. Two methods were used to detect the presence of energetic electrons: $K_{\alpha}$ flourescence of a metallic foil and 
direct detection using a focusing $90^{\circ}$ sector magnetic electron spectrometer. These experimental configurations are shown in figure 3.1 and discussed in section 3.4.

\subsection{1 $\mathrm{K}_{\alpha}$ Flourescence Measurement}

The first method used to measure energetic electrons was a $\mathrm{K}_{\alpha}$ flourescence technique, shown in figure 3.1(c). A schematic showing how the $\mathrm{K}_{\alpha}$ emission is generated is shown in figure 4.6. Electrons accelerated by the plasma wave which overcome the space charge effects of the plasma produce inner shell $(\mathrm{K})$ ionization, leading to emission characteristic of $\mathrm{K}_{\alpha} \mathrm{x}$-rays. In order to produce $\mathrm{K}_{\alpha}$ emission, the energy of the electron must be greater than the $\mathrm{K}_{\alpha} \mathrm{x}$-ray energy $(25.3 \mathrm{keV}$ for $\mathrm{Sn}, 17.5 \mathrm{keV}$ for Mo). Therefore, observation of $\mathrm{K}_{\alpha}$ emission is indicative of electrons with energies greater than the $\mathrm{K}_{\alpha}$ energy, $\mathrm{E} \geq \mathrm{E}_{\mathrm{K}_{\alpha}}$. The advantage to this technique is its simplicity. The primary disadvantage, however, is that an exact measurement of the energy of accelerated electrons can not be obtained. The measurement only provides a lower bound on the energy of the electrons (i.e., $\mathrm{E} \geq \mathrm{E}_{\mathrm{K}_{\alpha}}$ ). When this measurement was employed, the spectral features of forward SRS could not be simultaneously monitored, so correlations between energetic electrons and spectral forward SRS features could not be made.

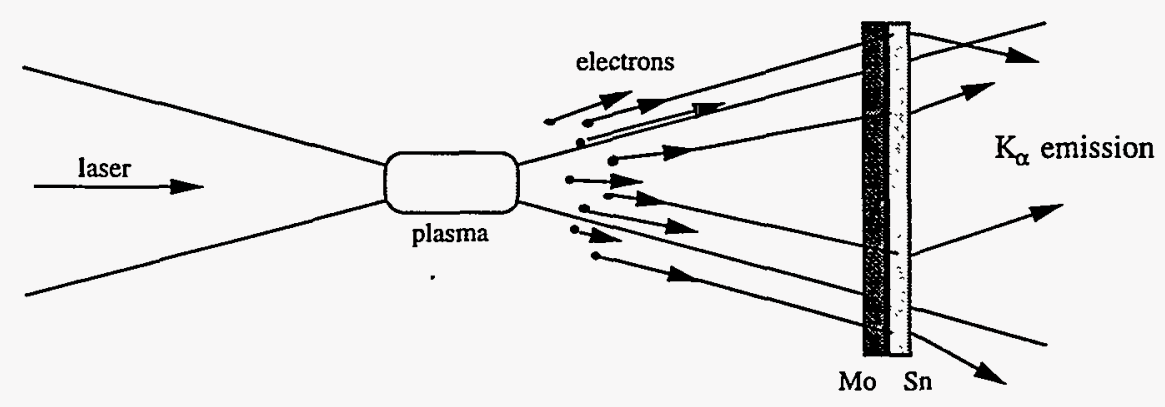

Figure 4.6: Schematic of the $\mathrm{K}_{\alpha}$ emission process

In the experiments, the 2 " $\times 2$ " metal foils which the electrons strike to produce $\mathrm{K}_{\alpha}$ emission were placed $10 \mathrm{~cm}$ from the focal region (plasma region) in the direct 
forward scatter direction on the focal axis. Experiments were performed using a 25 $\mu \mathrm{m}$ thick tin (Sn) foil, as well as a combination of $25 \mu \mathrm{m} \mathrm{Sn}$ and $50 \mu \mathrm{m}$ molybdenum (Mo). The Mo/Sn foil combination is shown in figures 3.1(c) and 4.6. The laser energies used in these $\mathrm{K}_{\alpha}$ flourescence experiments were $1-3 \mathrm{~J}$ and the gas jet plasma densities ranged from $\mathrm{n}_{0}=1 \times 10^{19}$ to $3 \times 10^{19} \mathrm{~cm}^{-3}$, the same parameters for which the best anti-Stokes spectral features were observed. The $\mathrm{K}_{\alpha} \mathrm{x}$-rays were observed as photon 'hits' on a silicon based CCD camera taking a 2-dimensional spatial picture. The number of counts in a particular pixel is proportional to the energy of the photon deposited (see section 3.4). Pulse-height analysis, i.e., an accounting of how many pixels had $K_{\alpha}$ energy deposited in them, produces an $x$-ray energy spectrum. The pulse-height analysis (histogram) procedure works only when there is a sufficiently low flux of $\mathrm{x}$-rays onto the CCD so that no pixel captures more than one photon. The CCD camera used to record the x-ray 'hits' was filtered with $1.3 \mathrm{~mm}$ of glass (window of CCD) and $0.76 \mathrm{~mm}$ of aluminum (visible light filter). The combined transmission of the glass and aluminum at $17.5 \mathrm{keV}$ (the $\mathrm{Mo} \mathrm{K}_{\alpha}$ energy) is $14 \%$. The transmission for the $\mathrm{Sn} \mathrm{K}_{\alpha}$ energy $\left(25.3 \mathrm{keV}\right.$ ) is $54 \%$ (glass and aluminum). The $\mathrm{Mo} \mathrm{K}_{\alpha} \mathrm{X}$-ray also sees $50 \mu \mathrm{m}$ of Mo and $25 \mu \mathrm{m}$ of $\mathrm{Sn}$, whereas the $\mathrm{Sn} \mathrm{K}_{\alpha}$ x-ray sees the $25 \mu \mathrm{m}$ of $\mathrm{Sn}$ as well as the glass and aluminum. Inclusion of these filtering effects reduces the Mo $\mathrm{K}_{\alpha}$ transmission to $3 \%$ and the $\mathrm{Sn} \mathrm{K}_{\alpha}$ transmission to $45 \%$.

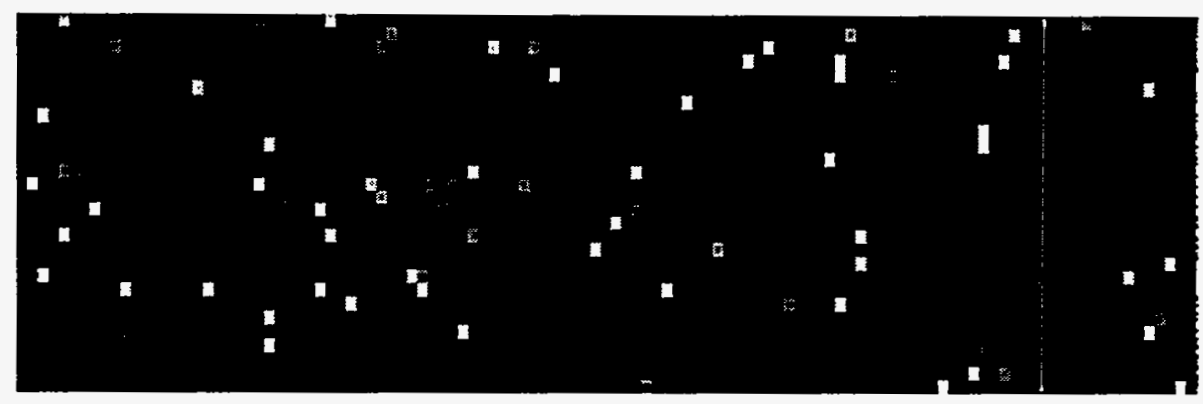

Figure 4.7: Example picture of the $\mathrm{K}_{\alpha} \mathrm{x}$-ray 'hits' recorded by the CCD.

Figure 4.7 shows a two-dimensional CCD image of the x-ray 'hits' obtained in a typical $\mathrm{K}_{\alpha}$ experiment. A pulse-height analysis of these 'hits' produces an $\mathrm{x}$-ray 
energy spectrum. Figure 4.8(a) shows a plot of the x-ray energy spectrum from a Sn target (laser $=2.3 \mathrm{~J}, 800 \mathrm{psi}$ helium backing pressure on the gas jet) and figure 4.8(b) shows the spectrum obtained from a Mo/Sn target (laser $=2.5 \mathrm{~J}, 800 \mathrm{psi}$ helium backing pressure on the gas jet). The pulse-height analysis shows, in both cases, a distinct peak near $25.3 \mathrm{keV}$, the $\mathrm{K}_{\alpha} \mathrm{x}$-ray energy for $\mathrm{Sn}$. No clear peak is observed above the noise at $17.5 \mathrm{keV}$ (Mo $\mathrm{K}_{\alpha}$ energy). The absence of the Mo $\mathrm{K}_{\alpha}$ peak probably results from the low transmission (3\%) of the Mo $\mathrm{K}_{\alpha} \mathrm{x}$-ray through the glass/aluminum/Mo/Sn filter. The amplitude of the $\mathrm{K}_{\alpha}$ peak was approximately the same for both targets.
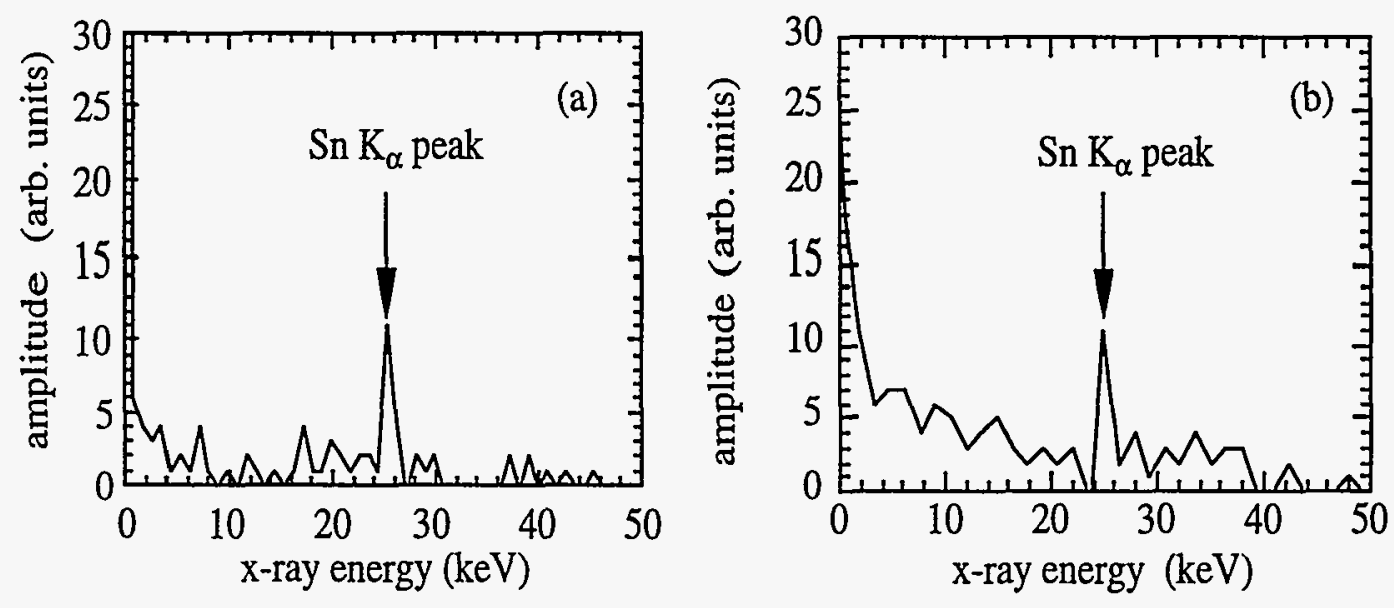

Figure 4.8: X-ray energy spectra obtained using (a) $25 \mu \mathrm{m}$ Sn and (b) $25 \mu \mathrm{m}$ Sn + $50 \mu \mathrm{m}$ Mo. The $\mathrm{K}_{\alpha}$ peak for $\mathrm{Sn}$ is at $25.3 \mathrm{keV}$.

A simple null test confirmed that the 'hits' observed on the CCD were indeed $\mathrm{K}_{\alpha}$ emission produced from energetic electrons and not the result of the intense laser pulse striking the metal foil. When no plasma was present to interact with the laser pulse, no photon 'hits' (i.e., $\mathrm{K}_{\alpha} \mathrm{x}$-ray emission) were observed.

If $50 \mu \mathrm{m}$ of Mo is approximately 1 practical range for the electron which produced the $\mathrm{Sn} \mathrm{K}_{\alpha}$ emission, then the energy associated with this electron would be approximately $700 \mathrm{keV}$. Since this thickness of Mo was used in the experiments, the only electrons which reach the $\mathrm{Sn}$ must have energies $\geq 700 \mathrm{keV}$. The electron plasma 
wave generated via stimulated Raman scattering can produce energetic electrons, as discussed in section 2.1. For backscattered SRS, the energy of electrons accelerated by the plasma wave is limited to a few $\mathrm{keV}$ for the parameters investigated here. Forward SRS, on the other hand, can produce electrons with much higher energies via wavebreaking ( $10 \mathrm{MeV}$ for the $\mathrm{n}=0.01 \mathrm{n}_{\mathrm{cr}}$ example of section 2.1). Therefore, since $\mathrm{K}_{\alpha}$ emission from $\mathrm{Sn}$ was observed after the electrons tranversed a $50 \mu \mathrm{m}$ Mo electron energy discriminator, which implies that $\mathrm{E}_{\text {electron }} \geq 700 \mathrm{keV}$, the energetic electrons observed could have only been generated via the forward Raman plasma wave.

An estimate for the number of energetic electrons which escaped from the plasma and reached the metal foil target can be made based on the number of x-ray 'hits' on the CCD. The CCD subtends a solid angle of approximately $2.5 \times 10^{-4}$. For $10 \mathrm{Sn}$ $\mathrm{K}_{\alpha}$ 'hits' present in the CCD image, the number of energetic electrons ( $E \geq 700 \mathrm{keV}$ ) which escaped the plasma is approximately $10^{6}$. If only $1 \%$ of the energetic electrons escape the plasma, then the total number of electrons in the plasma with energies greater than $700 \mathrm{keV}$ is approximately $10^{8}$.

In another experiment, only the electrons propagating outside the original $f / 8.2$ focal cone of the laser were collected. This was done using a 2 " $\times 2$ ", $25 \mu \mathrm{m}$ thick Sn foil with a $1.5 \mathrm{~cm}$ hole in the center, aligned such that the laser would propagate through the hole. This target was placed $10 \mathrm{~cm}$ beyond the plasma region so that the hole subtended an $f / 6.7$ cone. No appreciable change was observed in the $K_{\alpha}$ emission yield. This implies that most of the energetic electrons are scattered outside the focal cone of the laser.

To examine the angular region over which the electrons were scattered, a variation of the experimental setup of figure 3.1(c) was used. The metal foil target, a thin (0.3 inches) vertical strip of $50 \mu \mathrm{m}$ Mo on $25 \mu \mathrm{m} \mathrm{Sn}$, was positioned $10 \mathrm{~cm}$ from the plasma region on the focal axis, as before. In this experiment, however, two silicon surface barrier detectors were set up to look at the front side of the Mo, as shown in figure 4.9(a). These detectors are sensitive to $\mathrm{x}$-rays and electrons. Detector 

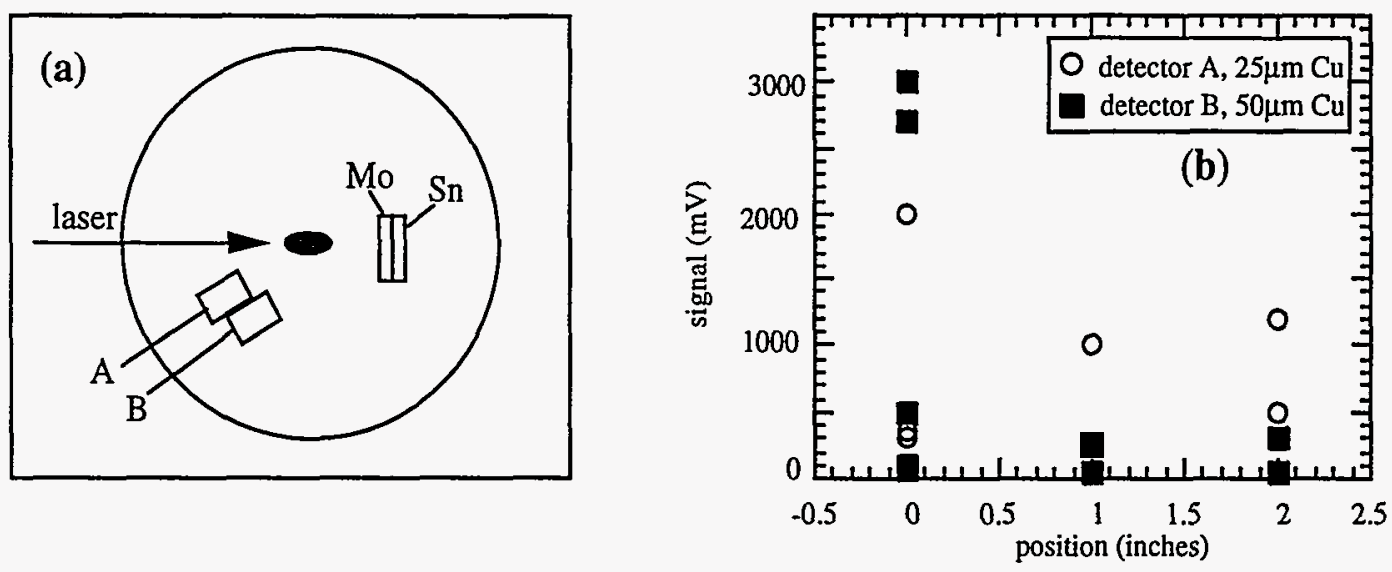

Figure 4.9: (a) Experimental setup used to study radial extent of the energetic electrons, and (b) $\mathrm{K}_{\alpha}$ emission as a function of radial distance from the laser axis.

"A" was filtered with $25 \mu \mathrm{m}$ of copper and detector "B" was filtered with $50 \mu \mathrm{m}$ of copper. A $25 \mu \mathrm{m}$ thick copper foil will not transmit $\mathrm{x}$-rays with energies below $\sim 5 \mathrm{keV}\left(\mathrm{T} \leq 10^{-2}\right)$ whereas for a $50 \mu \mathrm{m}$ copper foil the minimum observable $\mathrm{x}$-ray energy is approximately $10 \mathrm{keV}\left(\mathrm{T} \geq 10^{-2}\right)$. Therefore, the signal on the surface barrier detectors results from $\mathrm{x}$-rays with energies $>5 \mathrm{keV}$ (detector A) or $>10$ $\mathrm{keV}$ (detector $\mathrm{B}$ ). Since these $\mathrm{x}$-ray energies are much less than the $\mathrm{K}_{\alpha}$ energy of the metal foil, the $\mathrm{x}$-rays being collected with the detectors result from Bremsstrahlung radiation.

The signal levels of both detectors ( $\mathrm{A}$ and $\mathrm{B}$ ) were monitored at three positions of the metal target: on-axis, $1^{\prime \prime}$ off axis $\left(14^{\circ}\right)$, and $2^{\prime \prime}$ off axis $\left(27^{\circ}\right)$. These signal levels are plotted in figure $4.9(\mathrm{~b})$ as a function of position. It should be noted that the x-ray signal level may not fall off as rapidly as figure 4.9 (b) suggests since the signals in this plot have not been corrected for the sampling geometry. Clearly, the level of signal decreased as the distance from the axis increased. When the target was 1" from the laser axis, the signal level on the detector with stronger filtering (detector $B$ ) was in the noise. Since the signal rolls off with increasing distance from the laser axis, the signal cannot be the result of electrons striking the detectors and must result from 
Bremsstrahlung radiation. Since the level of Bremsstrahlung radiation is proportional to the flux of electrons, this implies that the most energetic electrons were scattered at angles $<14^{\circ}$.

\subsubsection{Magnetic Electron Spectrometer Measurement}

In order to obtain a more detailed measurement of the energetic electrons, and to simultaneously monitor energetic electrons and the forward SRS spectral features, a $90^{\circ}$ bending magnet was used. This magnet was on loan from the UCLA Plasma Accelerator Group. This experimental setup is shown in figure 3.1(d) and described in section 3.4.

In these experiments, the magnetic field was chosen such that $2.0 \mathrm{MeV}( \pm 0.1$ $\mathrm{MeV}$ ) electrons were directed to the electron surface barrier detector. This energy was chosen for two reasons. First, the maximum value of the magnetic field obtainable with the magnet used in these experiments is $2.7 \mathrm{kG}$ (power supply limited). This limits the maximum energy of electrons deflected to $4.0 \mathrm{MeV}$. Second, simulations ([37],[29]) show that $2.0 \mathrm{MeV}$ electrons should be present in substantial numbers in the electron energy distribution. Since only electrons which overcome the space-charge of the plasma will reach the deflecting magnet there must be many electrons accelerated in order to observe them in these experiments. The detector used to collect the electrons was shielded with $50 \mu \mathrm{m}$ of copper and surrounded by lead to minimize the $\mathrm{x}$-ray contribution to the signal, as discussed in section 3.4. The $\mathrm{x}$-ray signal on a control detector with identical shielding was simultaneously monitored and used to determine the x-ray contribution to the electron signal (see figure 3.1(d)). This contribution was typically about $10 \%$. The correlation between the signal levels on the control and electron detectors is shown in figure 4.10 (solid circles). As the signal level on the electron detector increased, the control detector signal increased as well.

A "null" experiment was performed to verify that the signal on the electron detector was indeed from electrons and not $\mathrm{x}$-rays. In this experiment, the polarity of 
the deflecting magnet was reversed, sending the electrons away from the electron surface barrier detector. Ideally, this should result in no signal on the electron detector. The "null" experiments are shown as open triangles. Clearly, the signals observed on the electron detector are significantly reduced compared to the signals obtained with the correct polarity on the magnet (for similar control detector values) in the "null" experiment.

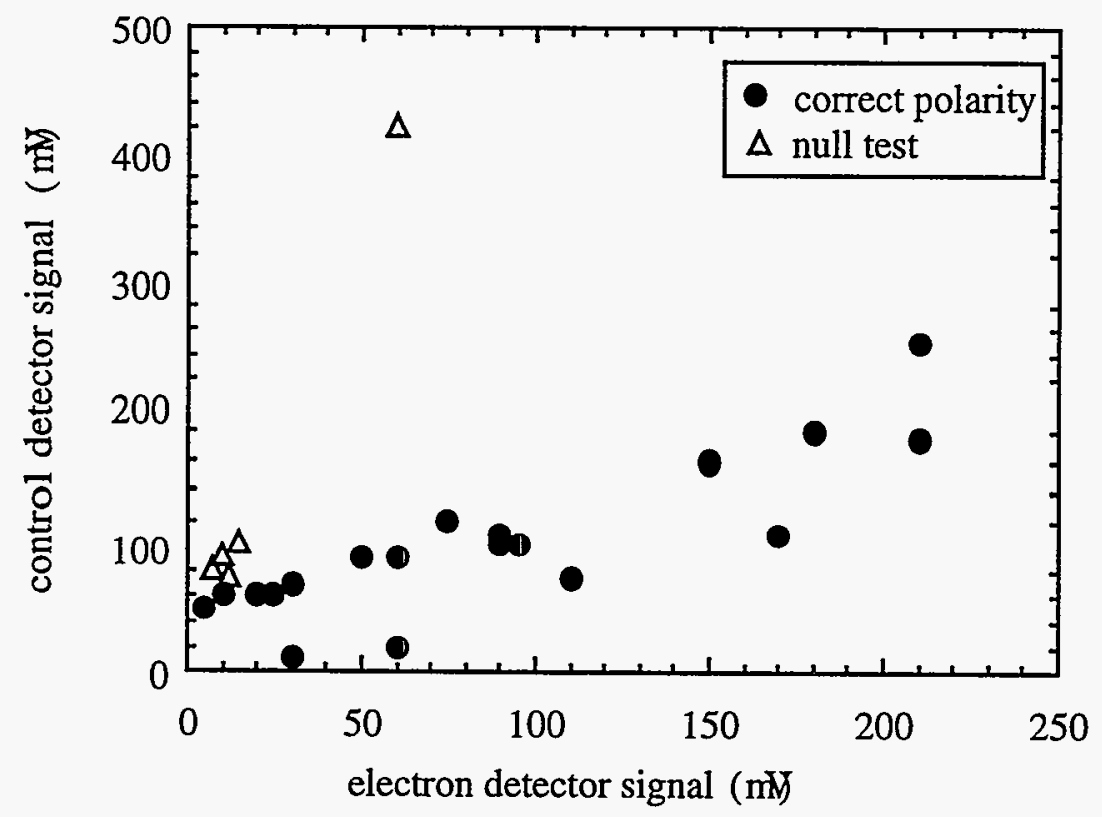

Figure 4.10: Signal levels on the electron and control detectors. The open triangles show the "null" experiment values.

On each laser shot, the near-forward spectra, the $2.0 \mathrm{MeV}$ electron signal, and the $\mathrm{x}$-ray signal were monitored. A distinct correlation between the electron signal and the amplitude of the first anti-Stokes feature was observed. This is shown in figure (4.11). These measurements were made at $800 \mathrm{psi}$, the pressure at which the antiStokes levels were typically highest. The laser energy ranged from $2 \mathrm{~J}$ to $3 \mathrm{~J}$. As the amplitude of the anti-Stokes increased by 4 orders of magnitude, the electron signal (corrected for the $\mathrm{x}$-ray contribution) was seen to generally increase over two decades (open circles). The $\mathrm{x}$-ray noise contribution (monitored by the control detector), 


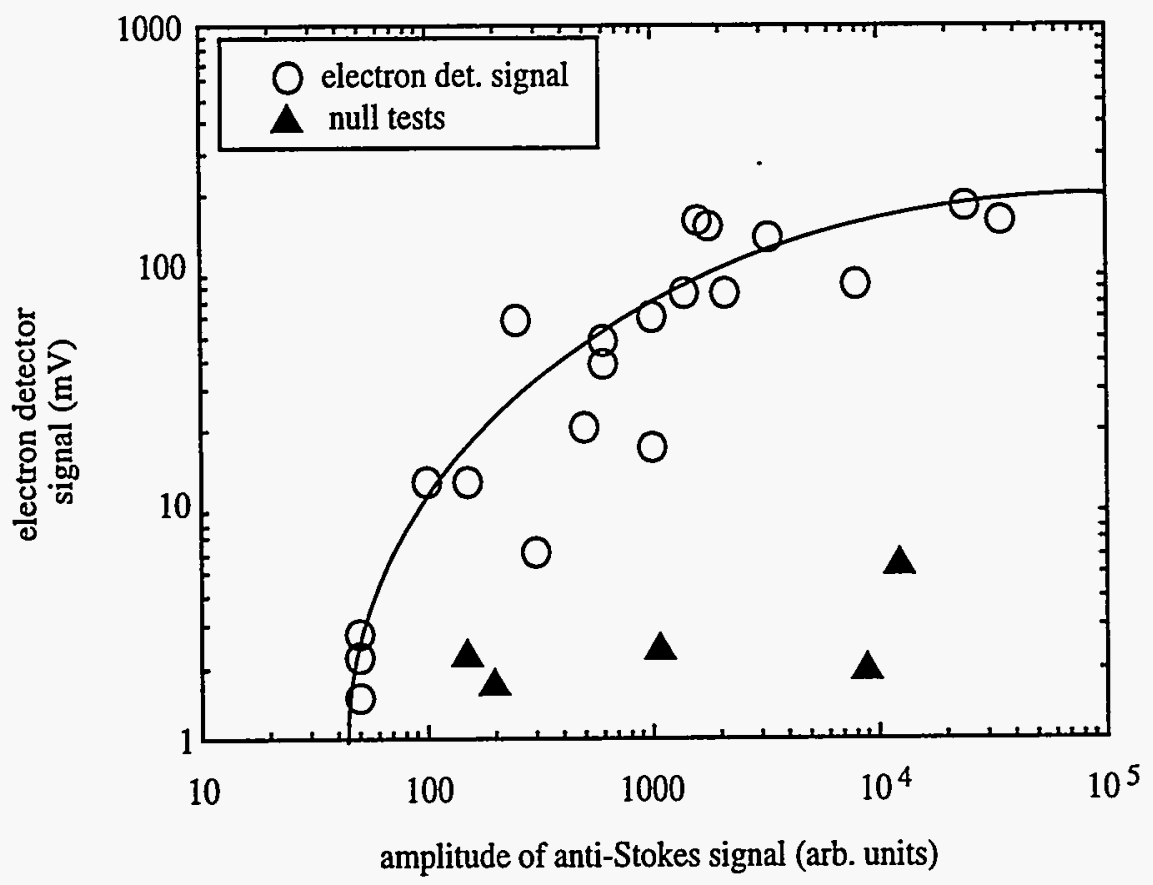

Figure 4.11: Correlation of electron signal with first anti-Stokes feature (open circles). The solid triangles indicate the "null" experiment.

on the other hand, remained nearly constant over the same range of anti-Stokes amplitude. When the polarity of the magnet was reversed (i.e., "null" experiment), no signal was observed on the electron detector. For this system, the total number of $2.0 \mathrm{MeV}$ electrons observed was below 50 (approximately $5-10 \mathrm{mV}$ per $2.0 \mathrm{MeV}$ electron). The obvious correlation between the observance of the anti-Stokes modes and the measurement of $2.0 \mathrm{MeV}$ electrons is clear evidence that forward SRS was occuring in the underdense plasma.

The 2.0 MeV electron signal varied with the gas jet backing pressure, as shown in figure 4.12. Clearly, as the gas jet backing pressure (i.e., plasma density) was increased, the level of electron signal increased despite some fluctuation in the electron signal. At the highest densities, however, the signal appears to decrease slightly. A similar decrease in the amplitude of the anti-Stokes feature was observed at high densities as well (see figure 4.4). The reduction in electron signal and the decrease in 


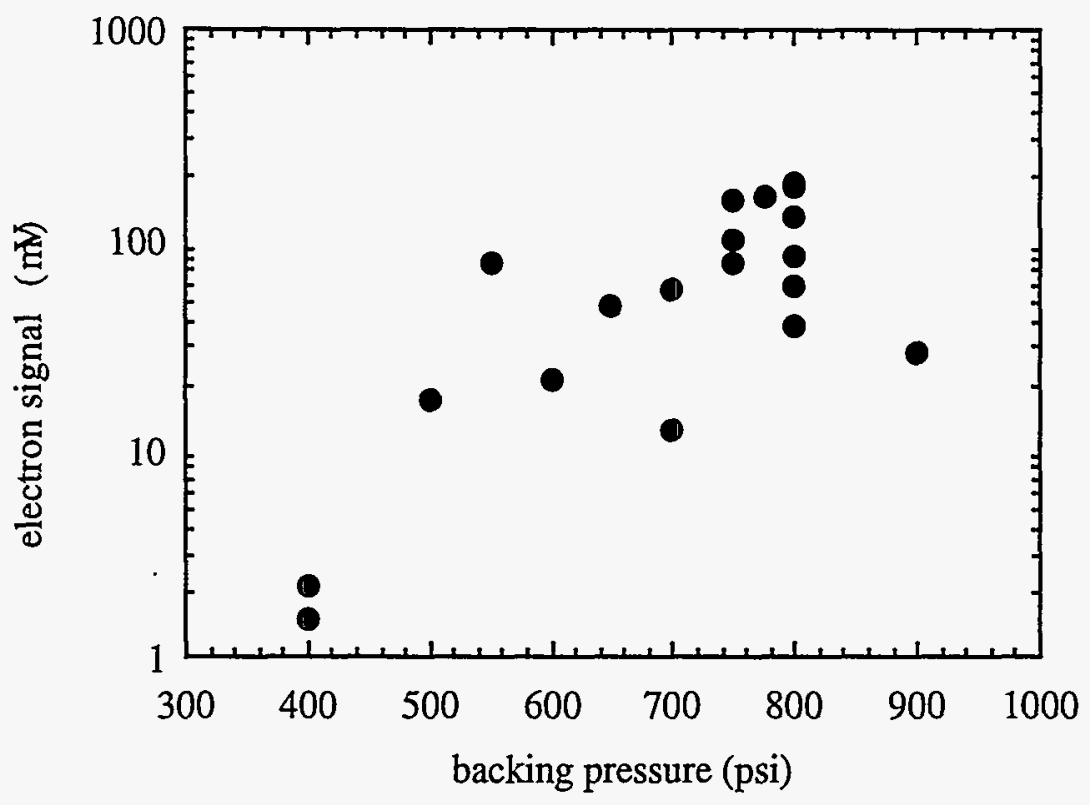

Figure 4.12: $2.0 \mathrm{MeV}$ electron signal as a function of gas jet backing pressure.

the anti-Stokes amplitude, combined with the correlation between the electron signal and the anti-Stokes amplitude shown in figure 4.11, indicate that the growth of the forward SRS was less than the growth obtained with lower plasma densities. This reduction in growth probably results from the reduced interaction length caused by ionization induced refraction and/or relativistic self-focusing.

\subsection{Energy transmitted through the underdense plasma}

To obtain information about how the generation of forward stimulated Raman scattering and its associated electrons affects the propagation of a short laser pulse through an underdense plasma, the amount of energy transmitted within the original $\mathrm{f} / 8.2$ cone of the laser was measured. Figure 4.13(a) shows the fraction of the incident laser energy transmitted through the focal cone as a function of incident laser energy. The open circles, solid triangles, and open squares were obtained at helium backing 
pressures of $400 \mathrm{psi}, 800 \mathrm{psi}$, and $1000 \mathrm{psi}$ respectively. At low incident laser energy $(<1 \mathrm{~J})$, the transmission was nearly unity for helium backing pressures of 400 and $800 \mathrm{psi}$. As the incident energy was increased beyond $1 \mathrm{~J}$, the fraction transmitted began to decrease. At the highest energy, only about $55 \%$ of the incident energy was transmitted in the cone angle of the laser beam for 800 psi. For a backing pressure of 400 psi (lower density), the transmission was $>80 \%$ except for energies greater than about $2 \mathrm{~J}$, where it dropped to approximately $65 \%$. At the highest densities (1000 psi backing pressure), the level of transmitted energy was less than $70 \%$ even at low incident laser energies. Only about $40 \%$ of the incident laser energy was transmitted through the underdense plasma in the original focal cone at the highest energy for a backing pressure of 1000 psi.

The decrease in the transmission of laser energy through the plasma correlated with the observance of the anti-Stokes feature, illustrated in figure 4.13(b). For both curves, the gas jet backing pressure was 800 psi of helium. As the level of incident light transmitted through the plasma decreased from approximately $100 \%$ to $55 \%$, the amplitude of the anti-Stokes feature increased by nearly 4 orders of magnitude. This strongly suggests that forward SRS (in a collisionless absorption or sidescatter process) is at least partially responsible for the reduction in transmission. Effects such as self-focusing and ionization induced refraction can scatter pump light out of the original focal cone. Massively parallel simulations (two-dimensional, fully relativistic particle-in-cell) [119], however, show that the generation of stimulated Raman forward scatter and sidescatter significantly reduce the amount of light transmitted through the plasma. These simulations were performed at UCLA by Warren Mori, Chris Decker, and Kuo-Cheng Tzeng using the specific parameters of these experiments. The decrease in transmitted light observed in the experiments agrees quantitatively with that observed in these one-to-one simulations. [29] Reference [29] has been included as appendix B. 
(a)
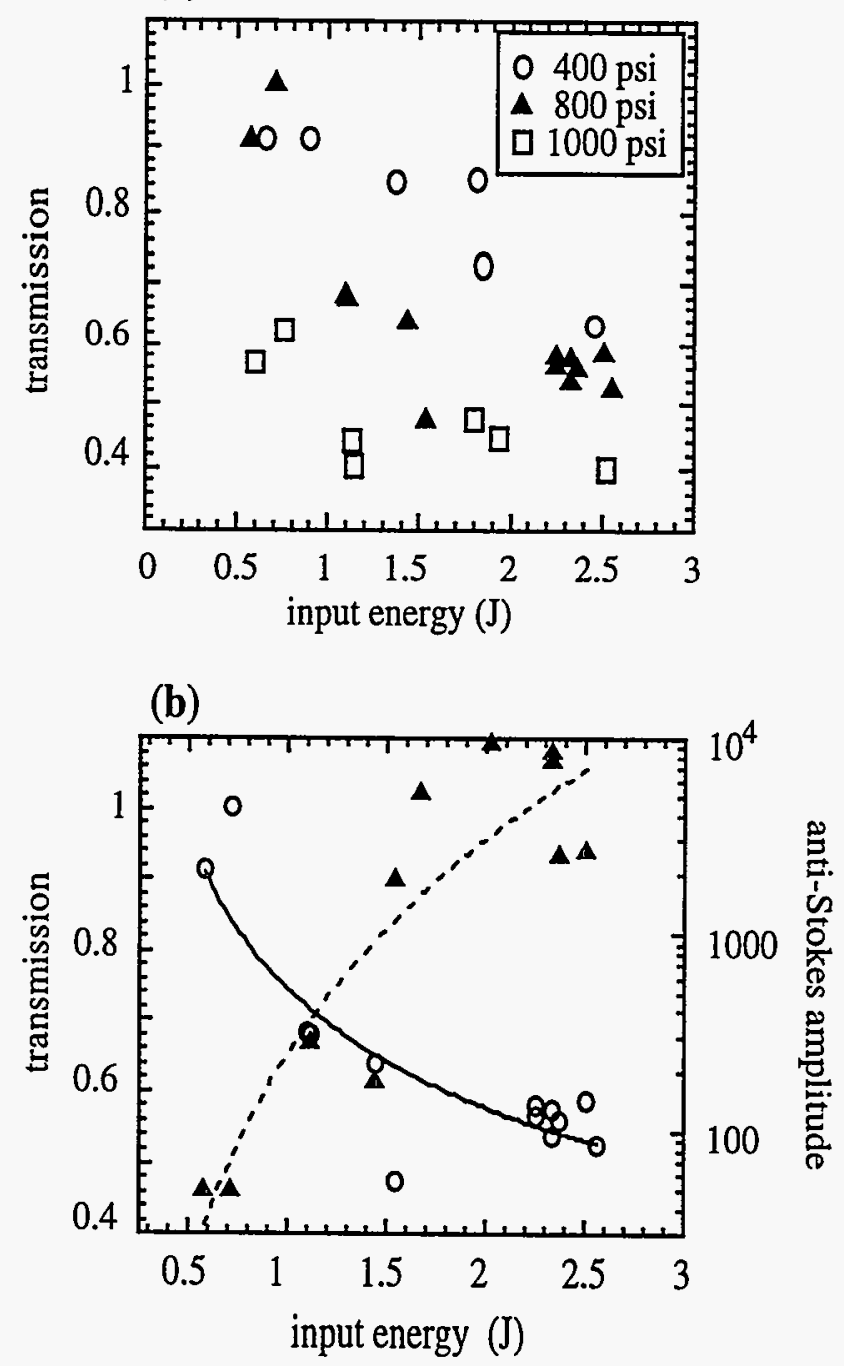

Figure 4.13: (a) Transmission of incident laser energy in the focal cone of the laser as a function of incident energy. (b) Correlation between the observation of anti-Stokes features and the decrease in transmission. 


\subsection{Implications of Forward SRS Observations}

The observation of forward stimulated Raman scattering from a short intense laser pulse interacting with an underdense plasma has some important implications. The observation of anti-Stokes features and energetic electrons, while interesting from a basic physics standpoint, merely corroborate simulation results $([37],[38],[84])$ that show it is possible to drive forward SRS in the short, intense laser pulse regime described in chapter 1 . In fact, the experimental results agree very well with the simulation predictions. The simulations predict the presence of energetic electrons and the observance of more than one anti-Stokes feature, both of which were observed in the experiments. The simulations also show that the presence of the second antiStokes feature is an indication that breakup of the laser beam is occuring. Therefore, given the agreement between simulation predictions and experimental observations, and the experimental observation of two anti-Stokes features, one might conclude that beam breakup is present in the experiments.

The correlation between the observation of the anti-Stokes modes and a decrease in the transmitted energy (figure 4.13(b)) also suggests that the propagation of the short pulse laser through an underdense plasma is seriously affected by the coupling of the laser pulse to the plasma via forward Raman scattering. The experimental observations and simulation results agree on this observation as well [29]; $55 \%$ of the incident light was transmitted through the plasma within the focal cone in the experiments whereas the simulations predicted $48 \%$. This remarkable agreement, discussed in reference [29], underscores the criticality of forward Raman scatter for propagation of intense short pulses in underdense plasmas. In fact, the PIC simulations show that longitudinal breakup of the laser beam can occur as a result of unchecked growth of forward SRS. The loss of the laser beam energy as the pulse propagates through an underdense plasma, and the potential for beam breakup, could seriously hinder applications which rely on the propagation of a short intense laser pulse through an underdense plasma channel. Two applications which rely on this are the laser wakefield accelerator ([107], [116]) and the fast ignitor fusion concept [115]. 


\subsection{Summary}

To summarize, stimulated Raman forward scatter was observed for the first time in short pulse, high intensity laser (600 fsec, $\mathrm{v}_{\mathrm{osc}} / \mathrm{c} \leq 0.9$ ), underdense plasma ( $\mathrm{n} \leq 3 \times 10^{19} \mathrm{~cm}^{-3}$ ) interactions. Two anti-Stokes features of the forward SRS were detected in the near-forward spectra. Energetic electrons typically associated with this instability were also observed with two techniques, $\mathrm{K}_{\alpha}$ flourescence and a deflecting magnet. Observations of $2 \mathrm{MeV}$ electrons correlated with observations of the anti-Stokes feature in the near forward direction. A decrease in the amount of light transmitted within the original focal cone of the laser was also observed and correlated with the appearance of forward SRS features, corroborating PIC simulation observations that foward Raman scatter adversely affects the propagation of a short pulse laser through an underdense plasma. 


\section{Chapter 5}

\section{Stimulated Raman Backscatter}

The results of experiments investigating stimulated Raman backscatter driven by an intense, subpicosecond laser are presented in this chapter. The parameter regime studied in the experiments is identified and general observations of backscattered SRS in this regime are discussed. Observations of anomalous spectral features in the backscattered spectra are also presented.

\subsection{Parameter regime}

In order for backscattered SRS to be distinguishable from the plasma noise in a calculation, there must be appreciable growth of the instability. I.e., $\gamma_{0} \tau \geq 2$ where $\gamma_{0}$ is the relativistic growth rate for Raman backscatter (equation (2.88)) and $\tau$ is the laser pulsewidth. This corresponds to a total gain of $e^{\gamma_{0} \tau} \geq 7.4$. Figure 5.1 illustrates the regime in which stimulated Raman backscatter can be driven with the laser system described in chapter $3 \cdot\left(\omega_{0}=1.79 \times 10^{15} \mathrm{sec}^{-1}, \tau=600 \mathrm{fsec}\right)$. The shaded area shows the densities and intensities for which the instability should be significantly above the plasma noise $\left(\gamma_{0} \tau \geq 2\right)$. The parameter regime experimentally achievable is also illustrated in figure 5.1. This region defines the parameter regime in which the SRS backscatter was studied. Specifically, the laser intensities ranged from $\mathrm{v}_{\mathrm{osc}} / \mathrm{c}=0.2$ to 0.8 and the plasma density was limited to $1 \times 10^{17} \mathrm{~cm}^{-3} \leq \mathrm{n}_{0} \leq 2 \times 10^{19} \mathrm{~cm}^{-3}$. 


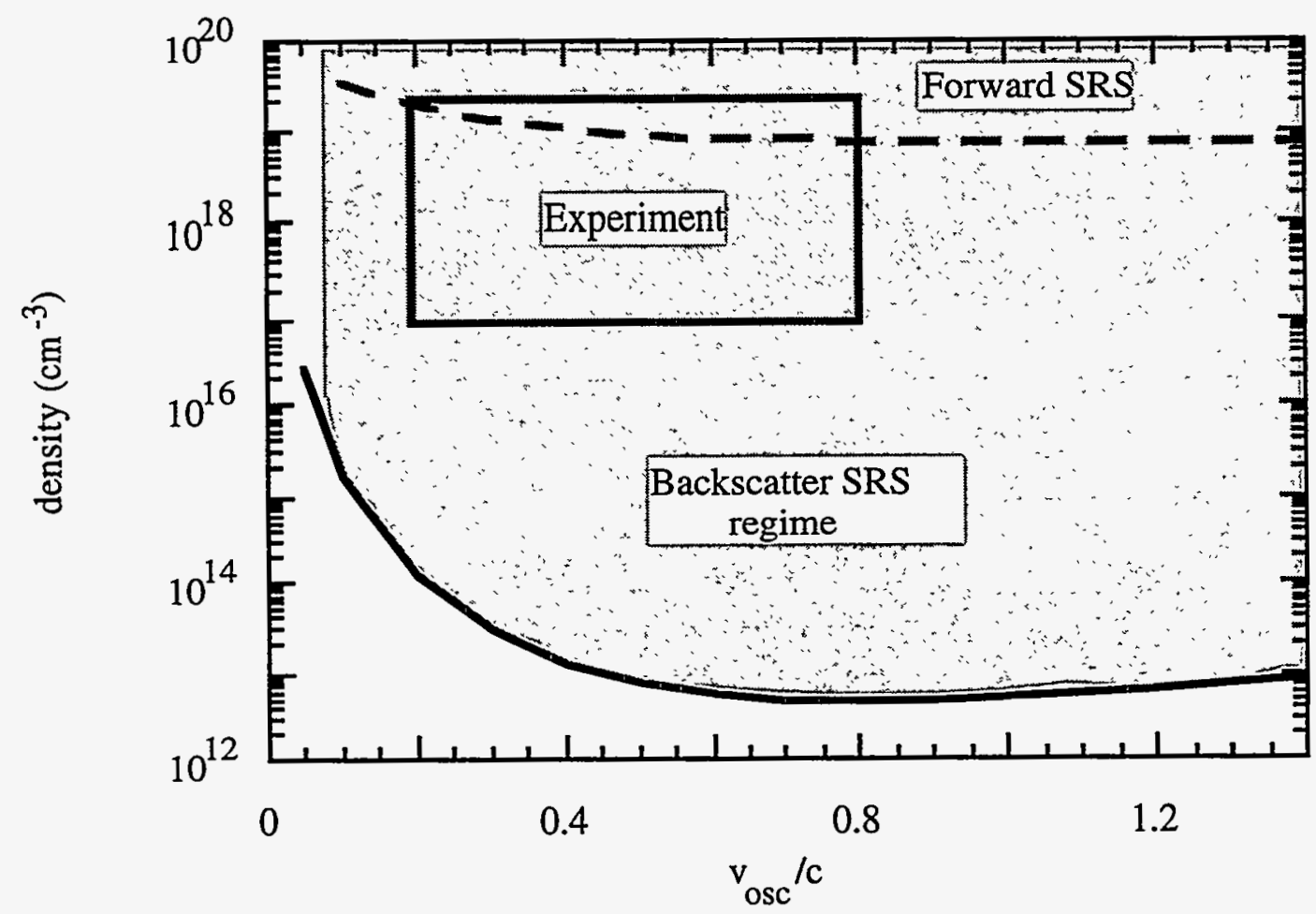

Figure 5.1: Density and intensity regime for which backscatterd stimulated Raman scatter can grow (shaded area). The dashed line defines the region in which forward SRS will be observable.

For plasma densities below $2 \times 10^{18} \mathrm{~cm}^{-3}$, a static fill of helium was used (see section 3.5). The plasmas created in the static fill ranged in size from about $1 \mathrm{~mm}$ to several millimeters, depending on the incident laser intensity. A gas jet was used to generate plasmas with densities greater than $5 \times 10^{18} \mathrm{~cm}^{-3}$. Helium was used as the target gas in these experiments as well. Plasmas produced with the gas jet were limited in size to approximately $1 \mathrm{~mm}$.

The total growth of backscattered SRS (number of e-foldings) ranged from $\gamma_{0} \tau=6$ to 15 for $\mathrm{n}_{0}=1 \times 10^{17} \mathrm{~cm}^{-3}$ and $\gamma_{0} \tau=34$ to 76 for $\mathrm{n}_{0}=1 \times 10^{19} \mathrm{~cm}^{-3}$. This level of growth corresponds to large gain of the stimulated Raman backscatter instability $\left(403-10^{33}\right)$. Clearly, this level of gain is unphysical, but the calculation used to generate these values does not include nonlinear saturation effects. 
Backscattered SRS is observable at much lower intensities and densities than forward scattered SRS. Comparison of the growth rates (equations (2.88) and (2.97)) shows that the growth rate for backscatter is $\left[\gamma\left(n_{c r} / n\right)\right]^{3 / 4}$ times larger than the forward scatter growth rate, where $\gamma$ is the relativistic correction factor, $\gamma=\sqrt{1+\mathrm{vosc}^{2} / \mathrm{c}^{2}}$. I.e.,

$$
\left(\gamma_{0}\right)_{\text {backscatter }}=\left[\gamma\left(\frac{n_{\text {cr }}}{n}\right)\right]^{\frac{3}{4}}\left(\gamma_{0}\right)_{\text {forward }}
$$

The dashed line in figure 5.1 shows the threshold above which foward SRS should be observable $\left(\gamma_{0} \tau \geq 1.4\right)$. For some of the experimental parameters, both stimulated Raman forward scatter and stimulated Raman backscatter should be excited.

The intensity and density necessary for the backscattered SRS to be experimentally observable is higher than figure 5.1 suggests. The expression $\gamma_{0} \tau \geq 2$ defines a region in which the SRS grows enough to be observed above the plasma noise level in the calculation. The minimum level of growth necessary for experimental detection is above the level determined by $\gamma_{0} \tau \geq 2$. This is the result of the configuration used to collect the BS light. In these experiments, the backscattered light is collected as leakage through the final turning mirror (see figure 3.1). A significant fraction of the light does not transmit through this optic, thereby increasing the minimum level of light necessary for SRS to be observable in these experiments. The sensitivity of the CCD camera used to record the data is reduced for wavelengths where backscattered SRS occurs. This poor sensitivity also increases the experimental threshold for observation of the instability. The experimental configuration used in these experiments is shown in figure 3.1 and described in section 3.3.

\subsection{General Behavior of Backscattered SRS}

In the experiments investigating stimulated Raman backscatter in the short pulse, high intensity laser, underdense plasma regime, modulated SRS backscatter spectra were observed. Figure (5.2) shows a series of backscattered spectra obtained for focal intensities ranging from $\mathrm{I}=5 \times 10^{16}$ to $3 \times 10^{17} \mathrm{~W} / \mathrm{cm}^{2}\left(\mathrm{v}_{\text {osc }} / \mathrm{c}=0.2\right.$ to 0.5$)$. At 
low intensity ( $\mathrm{v}_{\mathrm{osc}} / \mathrm{c}=0.2$ ), a laser fiducial (peak on the left) and a well-defined SRS feature were observed. The SRS feature was red-shifted relative to the laser fre-

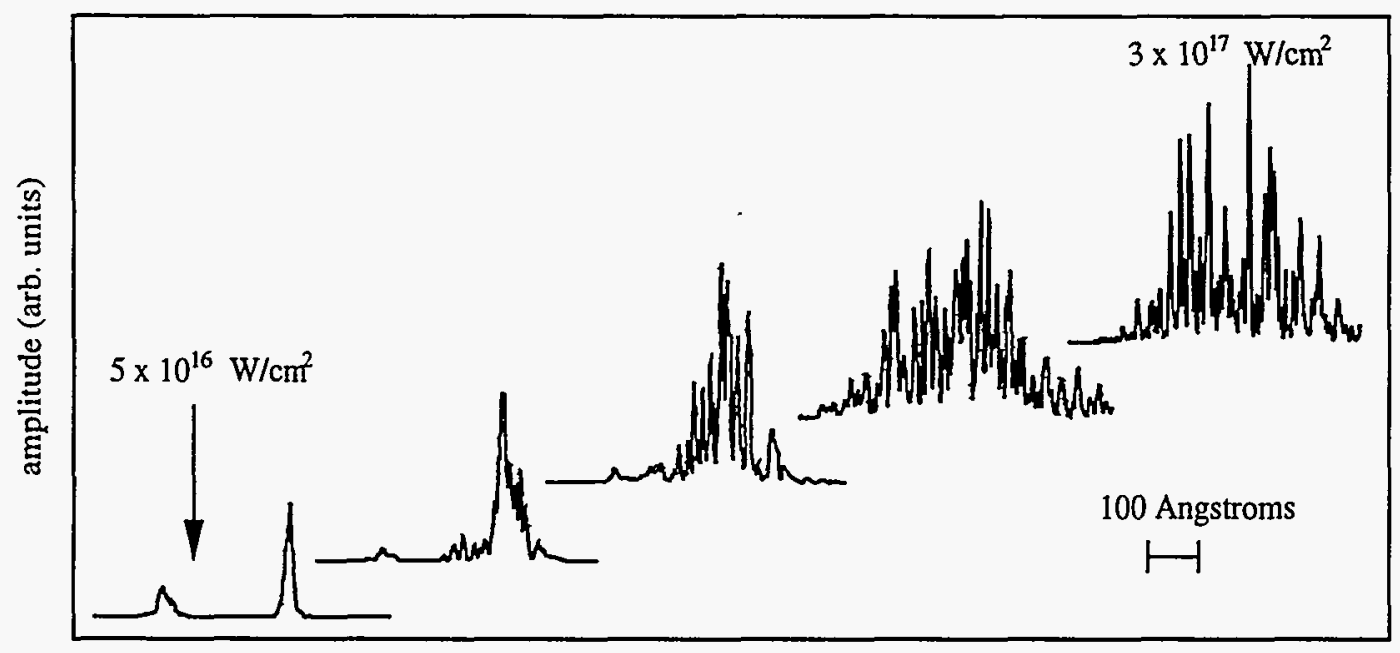

Figure 5.2: Backscattered stimulated Raman backscatter spectra obtained with plasma density of $\mathrm{n}_{0}=6 \times 10^{17} \mathrm{~cm}^{-3}$ and an intensity range of $5 \times 10^{16} \mathrm{~W} / \mathrm{cm}^{2}$ to $3 \times 10^{17} \mathrm{~W} / \mathrm{cm}^{2}$.

quency by approximately the plasma frequency for the known density ( 9 torr helium, $\left.6 \times 10^{17} \mathrm{~cm}^{-3}\right)$. The spectral width of this feature was essentially the same as that of the laser fiducial, $\Delta \lambda=32 \AA$. At a slightly higher intensity $\left(\mathrm{v}_{\text {osc }} / \mathrm{c}=0.28\right)$, the backscattered SRS feature began to broaden, as predicted by the dispersion relation. Further increases in power $\left(\mathrm{v}_{\mathrm{osc}} / \mathrm{c}>0.3\right.$ ) led to further broadening and severe spectral modulations of the feature. Concurrently, there was a gradual extension of the spectral energy toward the blue relative to the $\omega_{\mathrm{pe}}$-shifted SRS frequency obtained at low intensity. The modulated peaks became narrower and more closely spaced as the intensity was increased. This behavior was obtained throughout the density range studied.

A similar evolution of modulation and broadening in the backscattered spectra was observed as the plasma density was varied from $1.4 \times 10^{17} \mathrm{~cm}^{-3}$ to $1.3 \times 10^{18} \mathrm{~cm}^{-3}$, as shown in figure 5.3. The incident laser intensity was fixed at $1.7 \times 10^{17} \mathrm{~W} / \mathrm{cm}^{2}$ $\left(v_{\text {osc }} / c=0.37\right.$ ). As the density was increased, the SRS feature broadened and the 
degree of modulation increased. There was also a shift of spectral energy to the blue relative to the nominal $\omega_{\mathrm{pe}}$-shifted SRS frequency. As observed in the intensity evolution of the backscattered SRS, the individual peaks of the modulation were narrow and closely spaced at the higher densities.

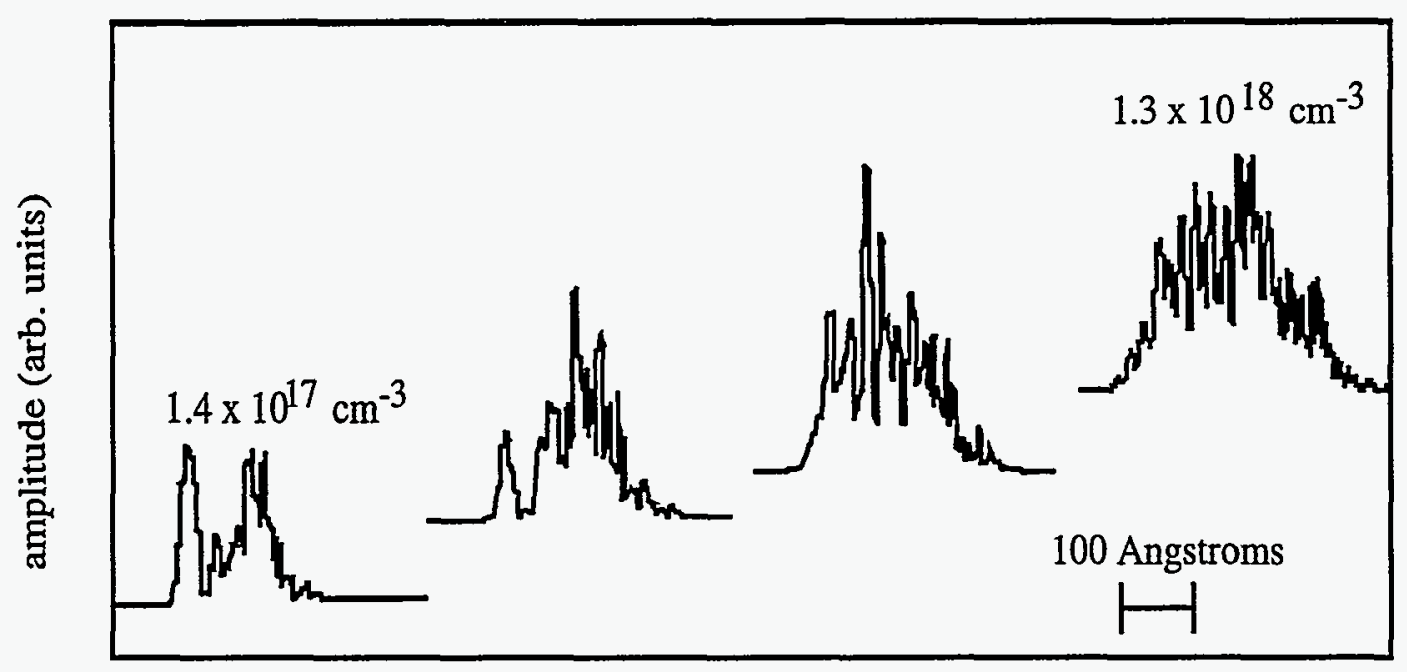

Figure 5.3: Backscattered SRS spectra obtained with plasma densities ranging from $1.4 \times 10^{17} \mathrm{~cm}^{-3}$ to $1.3 \times 10^{18} \mathrm{~cm}^{-3}$ and a fixed laser intensity of $1.7 \times 10^{17} \mathrm{~W} / \mathrm{cm}^{2}$.

\subsection{Spectral Features}

The stimulated Raman backscatter signals observed in the experiments exhibit both familiar and anomalous features. The familiar aspects of these spectra include the broadening of the spectra with intensity and the red shift of the SRS relative to the laser frequency. The dispersion relation solution presented in section 2.3 predicts a red-shifted SRS feature and broadening (see figure 2.7). Anomalous features in the spectra included the severe modulation and the blue-shifting behavior (relative to $\omega_{\mathrm{pe}}$ ) of the SRS feature. 


\subsubsection{Modulations}

\section{Individual Peaks}

The spectra shown in figures 5.2 and 5.3 show that the number of modulation peaks increased as the incident laser intensity and plasma density were increased. The widths of these modulations and the separations between adjacent modulations decreased as the plasma density and laser intensity increased. The individual modulations in the SRS backscatter feature were spectrally narrow, with widths ranging from 3 $10 \AA$. In contrast, the bandwidth of the laser is $3.2 \mathrm{~nm}$. The spectral resolution of the detector is approximately $0.5 \AA$. The narrowness of the individual modulations implies that they exist for several picoseconds, i.e., longer than the pulsewidth of the laser. The typical frequency separation between adjacent modulation peaks varied from about $\omega_{\text {pe }} / 10$ at low intensity to $\omega_{\text {pe }} / 30$ at the highest intensity. In many of the backscattered SRS spectra, there appeared, at first glance, to be some regularity present in the modulations. Closer analysis, however, revealed that a single period could not be identified. Furthermore, the locations of individual peaks in the modulated spectra were not reproducible on a shot-to-shot basis, as illustrated in figure 5.4. The three spectra in this plot were obtained sequentially with a fixed laser intensity of $2.5 \times 10^{17} \mathrm{~W} / \mathrm{cm}^{2}$ ( $\mathrm{v}_{\mathrm{osc}} / \mathrm{c}=0.45$ ) and plasma density of $6.7 \times 10^{17} \mathrm{~cm}^{-3}$. The overall shape and location of the spectrum is reasonably reproducible, but the locations of the individual modulations are not.

\section{Symmetric Behavior}

While there was no single repeating characteristic frequency separation present in the spectral modulations, further analysis in frequency space revealed a rather welldefined symmetric structure in many spectra. Specifically, a frequency "symmetry point" near the centroid of the SRS feature could often be defined such that for each modulation peak red-shifted relative to this symmetry point by an amount $\delta \omega$, there was a corresponding peak blue-shifted by nearly exactly the same $\delta \omega$. This is illustrated in figure 5.5(a), where a few of the "matching" modulation peaks are 


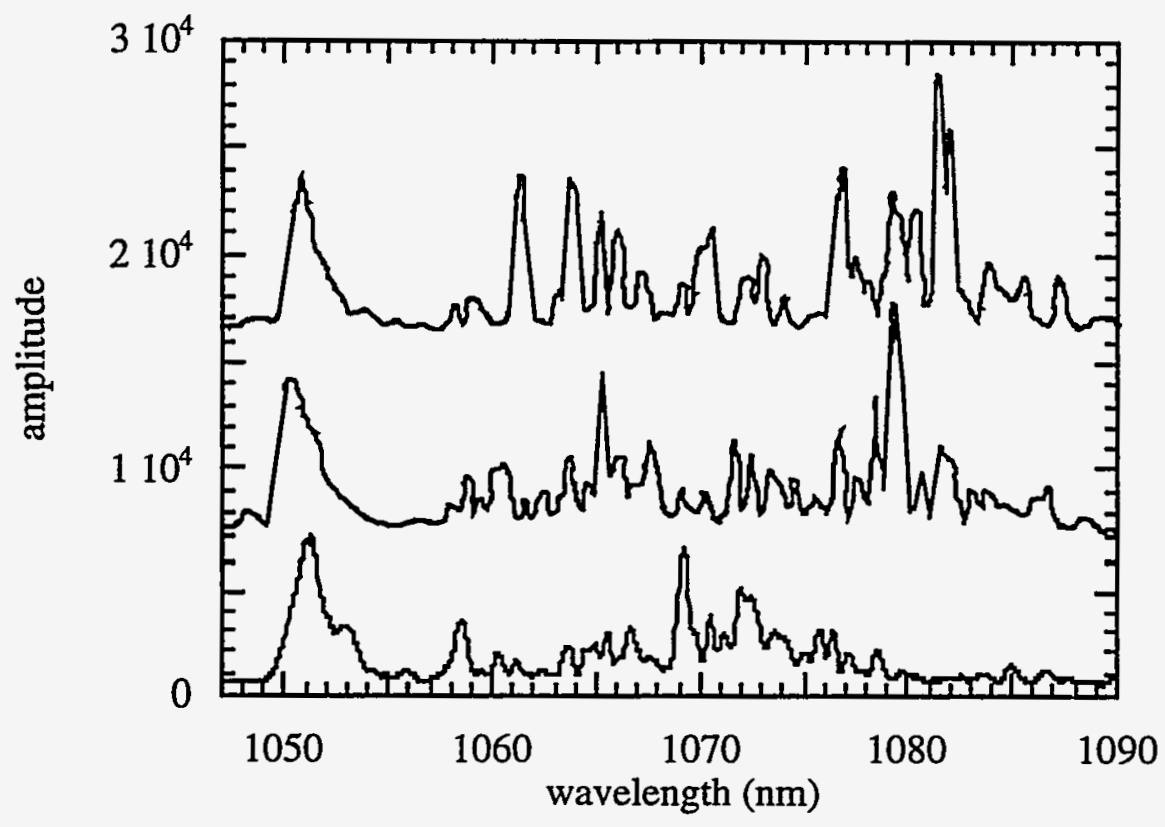

Figure 5.4: Three consecutive backscattered SRS spectra obtained for identical laser and plasma conditions. The laser intensity was $2.5 \times 10^{17} \mathrm{~W} / \mathrm{cm}^{2}$ and the plasma density was $6.7 \times 10^{17} \mathrm{~cm}^{-3}$.

identified. Figure 5.5(b) shows the absolute value of the frequency shifts of each modulation peak of figure 5.5(a) relative to the centriod frequency. The resulting points which represent these shifts lie on two $45^{\circ}$ lines since the shifts are linear with frequency. Symmetry is indicated when two points, one on each branch of the plot, fall on the same horizontal line. In this example, $70 \%$ of the red peaks had symmetric blue compliments which were exactly symmetric (within $0.02 \omega_{\text {pe }}(\sim 2 \AA)$ ). An additional $13 \%$ of the peaks were symmetric to within approximately $0.04 \omega_{\text {pe }}(\sim 5 \AA)$. The remaining $17 \%$ of the peaks exhibited no symmetry. Exact matches are illustrated by solid lines in figure 5.5(b) while close matches are shown with dashed lines. Note that out of the nineteen peaks (blue and red) closest to the symmetry point, fourteen were exact matches. The same analysis applied to other high intensity (modulated) spectra yields similar statistics with respect to symmetry. For lower intensity shots, an insufficient number of peaks existed for a meaningful analysis. 

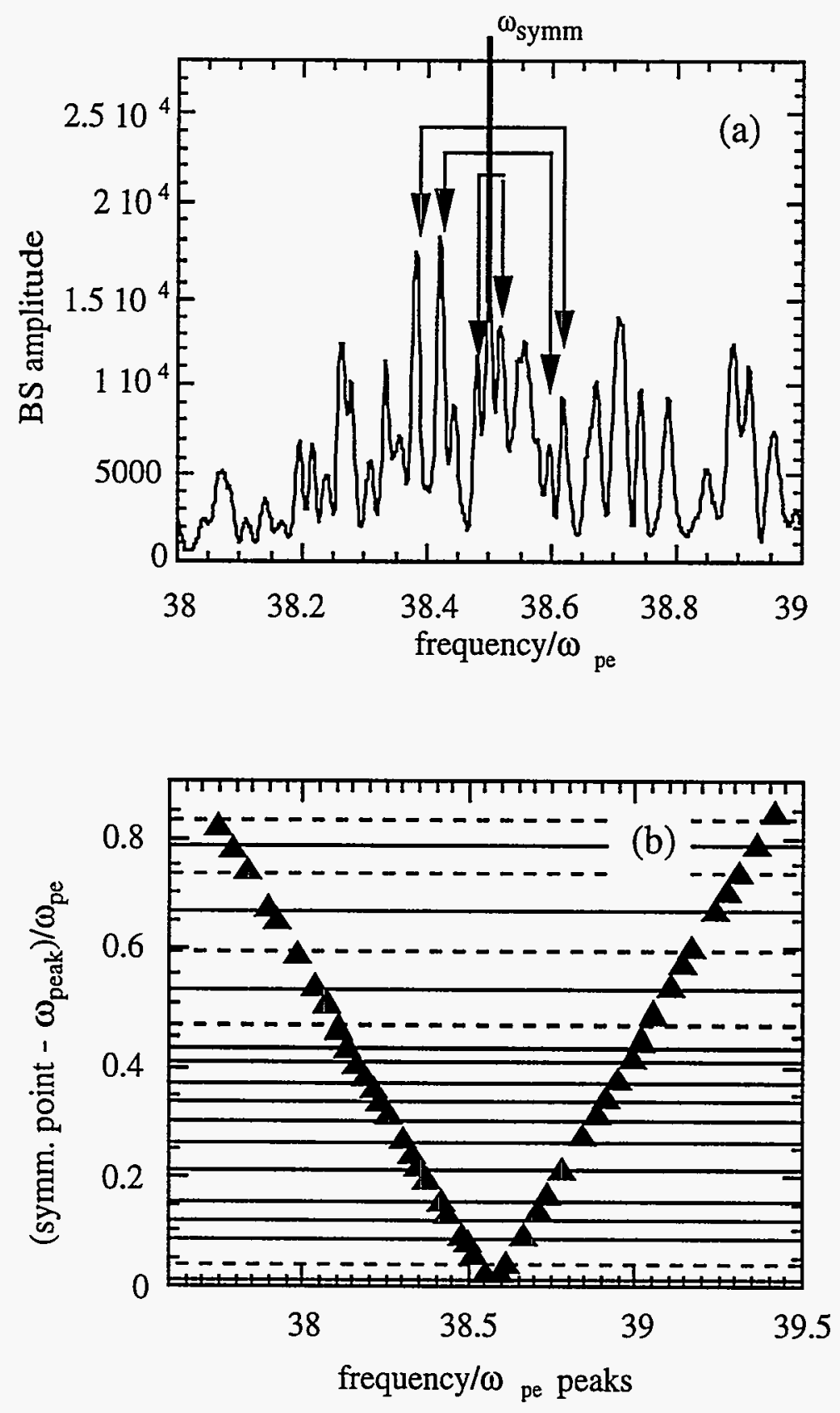

Figure 5.5: (a) Spectrum (in $\omega$-space) obtained for $\mathrm{n}_{0}=6 \times 10^{17} \mathrm{~cm}^{-3}$ and $\mathrm{v}_{\text {osc }} / \mathrm{c}=$ 0.46 , and (b) the corresponding symmetry of the modulation peaks. 


\subsubsection{Broad Feature Underlying the Modulations}

A broad Gaussian-like feature was observed to lie beneath the modulations in the SRS spectra. This feature resembles previously observed strongly coupled stimulated Raman backscatter [34], in which intensity broadening of the spectra and a gradual extension of spectral energy to the blue were observed. The dispersion relation, equation (2.87), can be used to predict the shape and location of the SRS feature in both the weakly coupled and strongly coupled regimes, as discussed in section 2.3. Comparison of predicted spectra and experimentally observed spectra show that the general shape of this underlying feature agrees with the calculated spectrum. Figure 5.6 shows the calculated (dashed line) and observed (solid line) spectra for $\mathrm{v}_{\mathrm{osc}} / \mathrm{c}=0.31$. The plasma density was $\mathrm{n}_{0}=6 \times 10^{17} \mathrm{~cm}^{-3}$. The spectrum obtained with the dispersion relation was multiplied by a factor appropriate to scale the calculated spectrum with the experimental result. The "detected" spectrum, shown by the
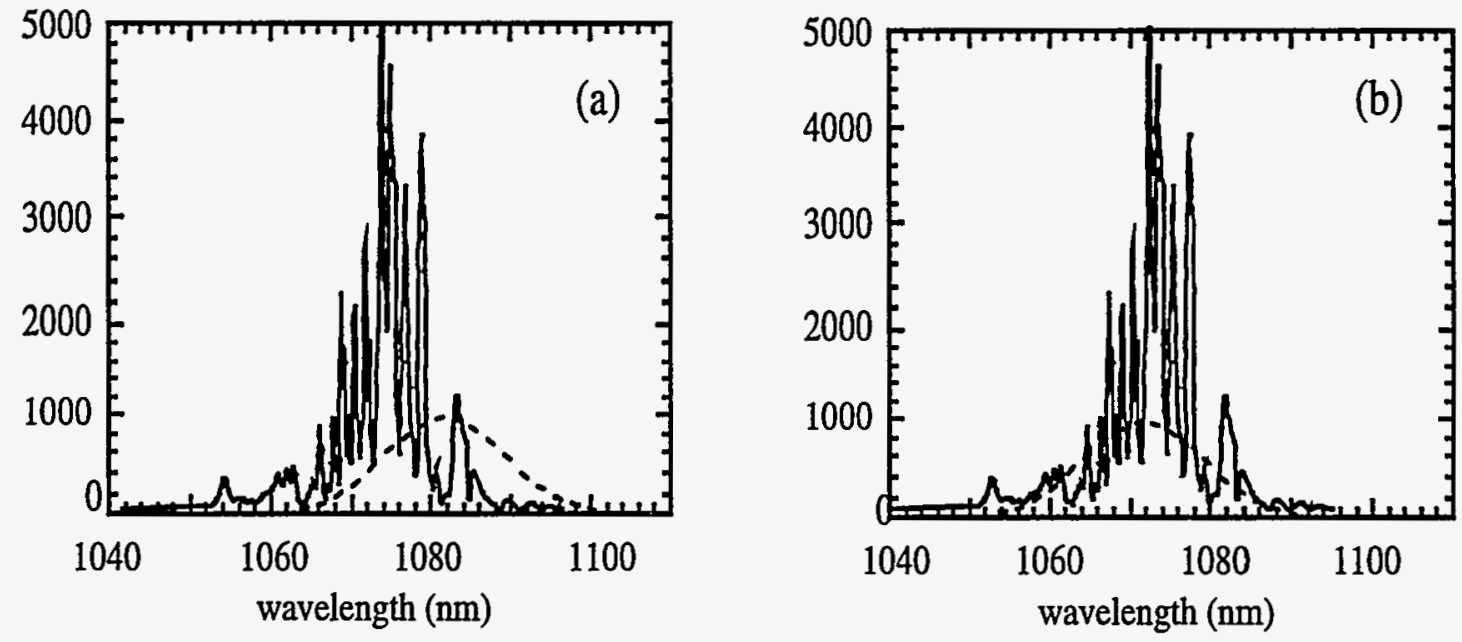

Figure 5.6: Calculated (dashed line) and experimentally observed (solid line) spectra for $v_{\text {osc }} / c=0.31$ and $n_{0}=6 \times 10^{17} \mathrm{~cm}^{-3}$. The "detected" spectrum is shown in (a) and the "shifted" spectrum is shown in (b).

dashed line in figure 5.6(a), was calculated using equation (2.96). The dashed line of figure $5.6(\mathrm{~b})$ is a "shifted" spectrum. The "shifted" spectrum is merely a wavelength 
shift of the "detected" spectrum to force the calculated spectrum to overlap the experimental spectrum. This shift is discussed later in this section. Clearly, the "shifted" spectrum accurately predicts the general shape of the broad feature underlying the modulations. Similar agreement was observed for other experimental spectra.

A variety of information about the SRS spectrum can be obtained from this broad feature, as shown in figure 5.7. The "width" of the SRS feature, obtained at the full

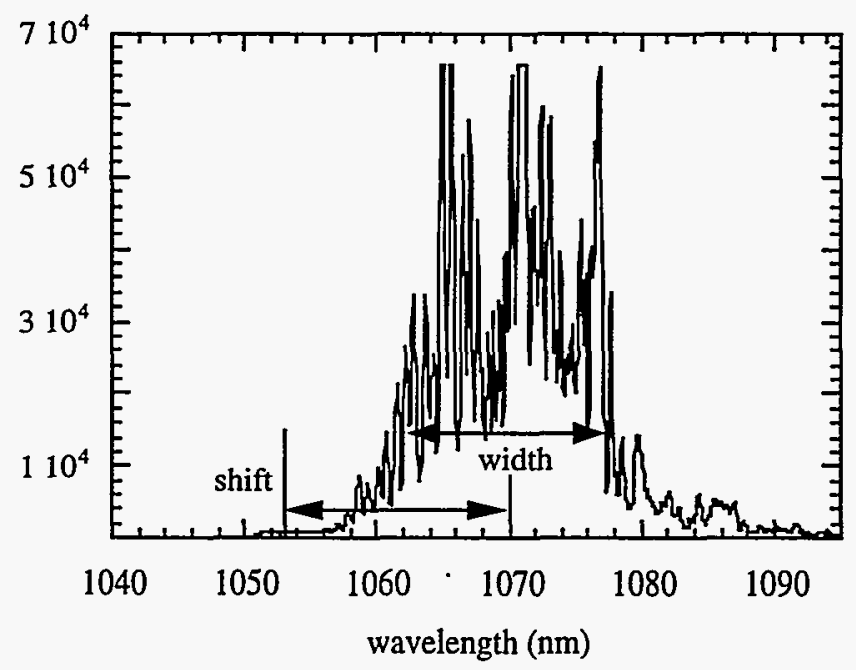

Figure 5.7: The "width" and "shift" of the SRS feature can be obtained from the backscattered spectrum.

width half-maximum, and the shift of the feature from the laser wavelength can be estimated from the experimental spectra. These results can then be compared to values obtained from the dispersion relation. The reflectivity of the stimulated Raman backscatter can also be measured by calculating the area of the SRS feature.

\section{Width}

Measurements of the full width at half-maximum of the broad feature underlying the modulations in the experimental spectra are shown in figure 5.8 (solid circles). These values were obtained for a plasma density of $\mathrm{n}_{0}=7 \times 10^{17} \mathrm{~cm}^{-3}$. Values of the width generated using the dispersion relation (equation (2.87)) are also shown in this figure (open squares). At low intensity, the SRS feature is unmodulated (see figure 5.2) 
and narrow $(<10 \mathrm{~nm})$. As the intensity increased, modulations appeared on the spectrum and the width of the feature increased. Extracting a width from the higher intensity spectra was difficult in many cases due the severity of the modulations. In general, the measured widths of the stimulated Raman backscatter feature were in reasonable agreement (order of magnitude) with the calculated results. Similar agreement was observed for other plasma densities. This agreement implies that the spectra calculated with the dispersion relation can be used to predict the general shape of the backscattered SRS spectra, even in the regime where modulations appear in the spectra.

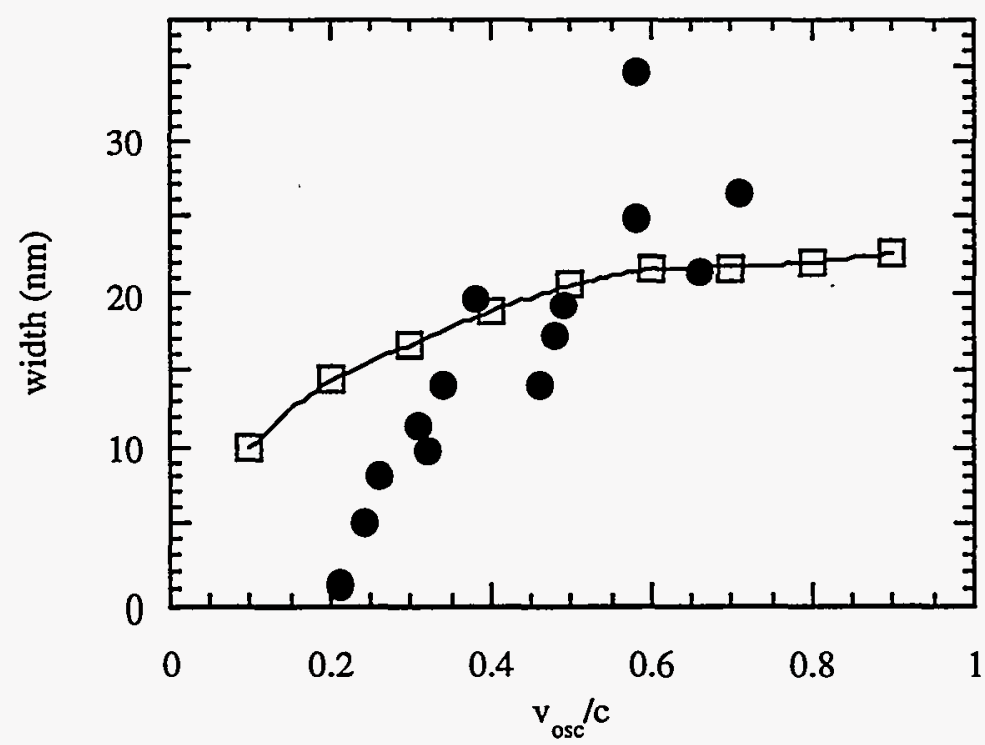

Figure 5.8: Full width half-maximum of the broad feature underlying the modulated SRS. The solid circles show experimentally measured widths for $\mathrm{n}_{0}=7 \times 10^{17} \mathrm{~cm}^{-3}$. The open squares were obtained from spectra calculated with the relativistic dispersion relation (equation (2.88)).

\section{Shift}

The shift of the stimulated Raman backscatter feature can also be obtained from the feature lying beneath the modulations, as shown in figure 5.7. For SRS, this 
shift should be approximately $\omega_{\mathrm{pe}}$. In the experiments performed here, however, there appeared to be a gradual shift of energy to the blue (relative to $\omega_{\mathrm{pe}}$ ) as the incident laser intensity was increased (see figure 5.2). Using equation (2.96), the expected location of the SRS feature can be found from the solution to the dispersion relation ("detected" spectrum). This location can then be compared with the shift observed experimentally in the backscattered spectra. As shown in figure 5.6, the calculated spectra needs to be shifted in wavelength in order to make it overlap with the experimentally observed spectrum ("shifted" spectrum). The distance by which the "detected" spectrum had to be "shifted" is plotted in figure 5.9 as a function of intensity for $\mathrm{n}_{0}=3 \times 10^{17} \mathrm{~cm}^{-3}$ (solid circles) and $\mathrm{n}_{0}=7 \times 10^{17} \mathrm{~cm}^{-3}$ (open triangles). At low intensity ( $\mathrm{v}_{\text {osc }} / \mathrm{c}<0.3$ ), the "detected" spectrum accurately predicts the location of the observed spectrum. At higher intensities, however, the "shift" of the spectrum increases, particularly for the higher density plasma. At the highest intensity $\left(\mathrm{v}_{\text {osc }} / \mathrm{c}=0.71\right)$, the "detected" spectrum needs to be shifted by almost 12

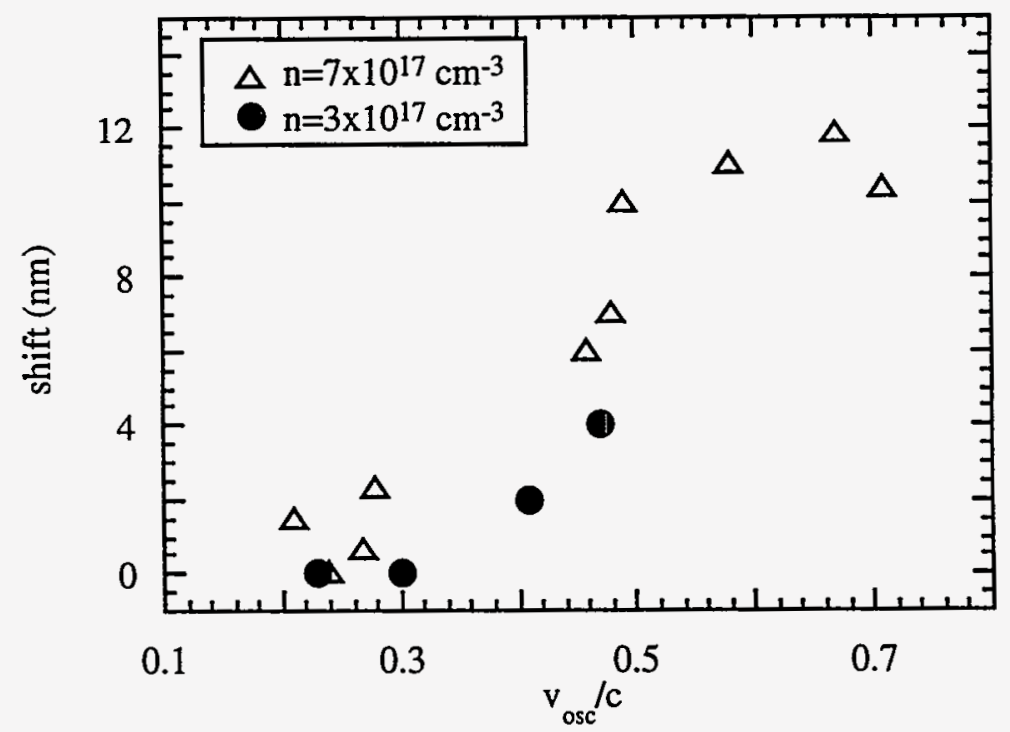

Figure 5.9: Distance the calculated spectra must be blue-shifted in order to overlap the experimentally observed spectra. The solid circles (open squares) show the shift for $\mathrm{n}_{0}=3 \times 10^{17} \mathrm{~cm}^{-3}\left(7 \times 10^{17} \mathrm{~cm}^{-3}\right)$. 
$\mathrm{nm}$ in order for the calculated and observed spectra to agree. This necessary blueshift does not agree with standard stimulated Raman backscatter theory, and has yet to be adequately explained. Potential mechanisms for these anomalous features are discussed in section 5.4.

\subsubsection{Reflectivity}

The reflectivity of the stimulated Raman backscatter was also measured in the experiments. To obtain these values, the spectra were recorded, the spectral response of the backscatter path calibrated, and the energy was obtained by integrating the SRS spectrum $\left(\int \frac{\mathrm{dP}}{\mathrm{d} \lambda} \mathrm{d} \lambda\right)$. This method slightly underestimates the actual reflectivity since light at exactly the laser wavelength was used to calibrate the backscatter path and detector. Because the detector is less sensitive for the wavelengths typically associated with SRS (i.e., red side of $\omega_{0}$ ) than it is for the laser wavelength. Therefore the calibration factor, $\frac{d E}{d \lambda}$, will underestimate the amount of energy in the spectral feature. This technique for calculating the reflectivity is accurate to within a factor of 5 , however.

Figure 5.10 shows a plot of the measured reflectivity as a function of intensity for two plasma densities, $\mathrm{n}_{0}=3 \times 10^{17}$ (open triangles) and $7 \times 10^{17} \mathrm{~cm}^{-3}$ (solid circles). The reflectivity increases intially with intensity and appears to reach a saturation level of approximately $10^{-4}$ near $\mathrm{v}_{\text {osc }} / \mathrm{c}=0.4$. The peak reflectivity observed for a plasma density of $\mathrm{n}_{0} \sim 7 \times 10^{17} \mathrm{~cm}^{-3}$ was $2 \times 10^{-4}$ (i.e., $0.02 \%$ of the incident laser light was collected as stimulated Raman backscatter in the solid angle subtended by the focus lens). Reflectivity values obtained for several different plasma densities exhibit similar peak reflectivities and saturation behavior. For a density of $\mathrm{n}_{0} \approx 10^{18} \mathrm{~cm}^{-3}$, the peak reflectivity was also near $2 \times 10^{-4}$ with the reflectivity saturating at about $\mathrm{v}_{\text {osc }} / \mathrm{c}=0.35$. It should be noted that the $\mathrm{v}_{\text {osc }} / \mathrm{c}$ at which the reflectivity saturates is approximately the same as the $\mathrm{v}_{\mathrm{osc}} / \mathrm{c}$ at which the modulations appear (see section 5.2). The saturation of the reflectivity indicates that nonlinear effects are occuring in the plasma. I.e., the SRS is no longer growing in the linear regime. The saturation 


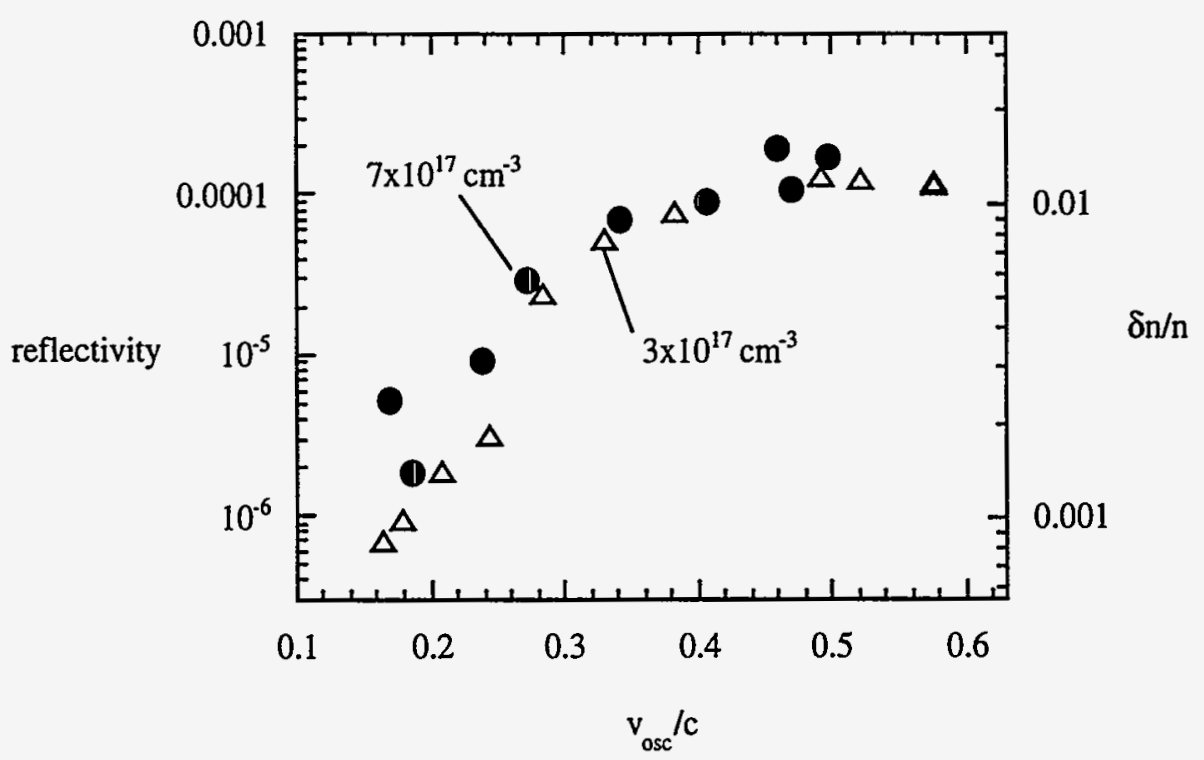

Figure 5.10: Reflectivity of the backscattered SRS feature as a function of incident laser intensity. The open triangles (solid circles) show the reflectivity values for $\mathrm{n}_{0}=3 \times 10^{17} \mathrm{~cm}^{-3}\left(7 \times 10^{17} \mathrm{~cm}^{-3}\right)$ with $\mathrm{L}_{\mathrm{z}}=1 \mathrm{~mm}$. The density fluctuation, $\delta \mathrm{n} / \mathrm{n}$ is given on the right hand $\mathrm{y}$-axis.

level of the reflectivity also indicates that the presence of backscattered SRS in the plasma does not dramatically pump deplete the laser. The peak reflectivity is only a fraction of a percent $(0.02 \%)$, implying that little laser energy is lost in the production of backscattered SRS.

Estimates for the initial density flucutation, $\delta \mathrm{n} / \mathrm{n}$, can be obtained from the reflectivity using [104]

$$
\mathrm{R}=\frac{\mathrm{P}_{\mathrm{s}}}{\mathrm{P}_{0}}=\frac{1}{4}\left(\frac{\delta \mathrm{n}}{\mathrm{n}_{0}}\right)^{2} \mathrm{r}_{0}^{2} \lambda^{2} \mathrm{~L}_{\mathrm{z}}^{2} \mathrm{n}_{0}^{2}
$$

where $\lambda$ is the laser wavelength, $r_{0}^{2}$ is the Thomson cross section $\left(r_{0}=e^{2} / \mathrm{mc}^{2}\right)$, and $\mathrm{L}_{\mathrm{z}}$ is the length of the plasma. Values for the density fluctuation are also plotted in figure 5.10 as a function of intensity for $n_{0}=3 \times 10^{17} \mathrm{~cm}^{-3}$ and $7 \times 10^{17} \mathrm{~cm}^{-3}$ (right side $y$-axis). The right hand axis of figure 5.10 shows that as the reflectivity of the backscattered SRS increased, the amplitude of the density perturbation necessary to produce the SRS increased as well. For $\mathrm{v}_{\mathrm{osc}} / \mathrm{c}=0.46$ and $\mathrm{n}_{0}=7 \times 10^{17} \mathrm{~cm}^{-3}, \delta \mathrm{n} / \mathrm{n}$ 
was 0.03 , its largest value. Table 5.1 lists several values of $\delta \mathrm{n} / \mathrm{n}$, obtained for a variety of densities and intensities in the experiments.

The growth rate of backscattered SRS can be obtained from the reflectivity, R. The reflectivity is given by

$$
\mathrm{R}=\frac{\mathrm{E}_{\mathrm{n}}}{\mathrm{E}_{0}} \mathrm{e}^{\gamma_{0} \tau}
$$

where $\mathrm{E}_{\mathrm{n}}$ is proportional to the noise power from which the SRS grew, $\mathrm{E}_{0}$, the laser energy scattered by the noise level, is proportional to the power incident on the plasma, and $\gamma_{0} \tau$ is a constant for a fixed density and laser intensity. Equation (2.88) shows that the growth rate, $\gamma_{0}$, is linearly related to the oscillation velocity, $v_{\text {osc }} / \mathrm{c}$. In particular,

$$
\gamma_{0} \tau=\kappa \frac{\mathrm{v}_{\text {osc }}}{\mathrm{c}}\left(1+\frac{\mathrm{v}_{\text {osc }}{ }^{2}}{\mathrm{c}^{2}}\right)^{-\frac{5}{8}}
$$

where $\kappa$ is a constant. If the reflectivity is known at more than one energy (for a fixed plasma density), the linear growth rate can be calculated for each incident energy. The growth rate as a function of $v_{\text {osc }}$ is given by

$$
\gamma_{\mathrm{ref}}=\frac{\kappa \mathrm{v}_{\text {osc }}}{\tau}\left(1+\frac{\mathrm{v}_{\text {osc }^{2}}}{\mathrm{c}^{2}}\right)^{-\frac{5}{8}}
$$

where $\kappa$ is a constant. $\kappa$ can be found by taking the ratio of two reflectivity values. I.e.,

$$
\left.\frac{R_{1}}{R_{2}}=\frac{E_{02}}{E_{01}} e^{\left(\gamma_{1}-\gamma_{2}\right.}\right) \tau
$$

based on equation (5.3). $\gamma_{1}$ and $\gamma_{2}$ can be written as $\gamma_{1} \tau=\kappa\left(\frac{v_{\text {oscl }}}{c}\right)\left(1+\mathrm{v}_{\text {osc1 }}{ }^{2} / \mathrm{c}^{2}\right)^{-\frac{5}{8}}$ and $\gamma_{2} \tau=\kappa\left(\frac{\mathrm{v}_{\text {osc }}}{\mathrm{c}}\right)\left(1+\mathrm{v}_{\mathrm{osc} 2}{ }^{2} / \mathrm{c}^{2}\right)^{-\frac{5}{8}}$, the expression above can be rearranged to give

$$
\kappa=\frac{1}{v_{\text {osc1 }}\left(1+\frac{v_{\text {osc } 12} c^{2}}{c^{2}}\right)^{-\frac{5}{8}}-v_{\text {osc2 }}\left(1+\frac{\left.v_{\text {osc } 2}\right)^{2}}{c^{2}}\right)^{-\frac{5}{8}}} \ln \left[\left(\frac{R_{1}}{R_{2}}\right)\left(\frac{v_{\text {osc1 }}}{v_{\text {osc2 }}}\right)^{2}\right]
$$

since $\mathrm{E}_{0 \mathrm{i}} \propto\left(\mathrm{v}_{\mathrm{osci}}\right)^{2}$. Figure 5.11 shows the growth rate obtained from the reflectivity (solid circles) as a function of $\mathrm{v}_{\text {osc }} / \mathrm{c}$. The reflectivity values used were those for $\mathrm{n}_{0}=7 \times 10^{17} \mathrm{~cm}^{-3}$, as shown in figure 5.10. The result obtained with equation 


\begin{tabular}{||l|l|l||}
\hline plasma density & $\mathrm{v}_{\text {osc }} / \mathrm{c}$ & $\delta n / n$ \\
\hline $3 \times 10^{17} \mathrm{~cm}^{-3}$ & 0.25 & 0.0018 \\
\cline { 2 - 4 } & 0.38 & 0.009 \\
\cline { 2 - 4 } & 0.49 & 0.012 \\
\hline \multirow{3}{*}{$7 \times 10^{17} \mathrm{~cm}^{-3}$} & 0.19 & 0.0013 \\
\cline { 2 - 4 } & 0.34 & 0.0086 \\
\cline { 2 - 4 } & 0.46 & 0.014 \\
\hline \multirow{2}{*}{$1 \times 10^{18} \mathrm{~cm}^{-3}$} & 0.27 & 0.022 \\
\cline { 2 - 4 } & 0.55 & 0.076 \\
\hline
\end{tabular}

Table 5.1: Values of the density fluctuation, $\delta \mathrm{n} / \mathrm{n}$, for a variety of plasma densities and incident laser intensities. 


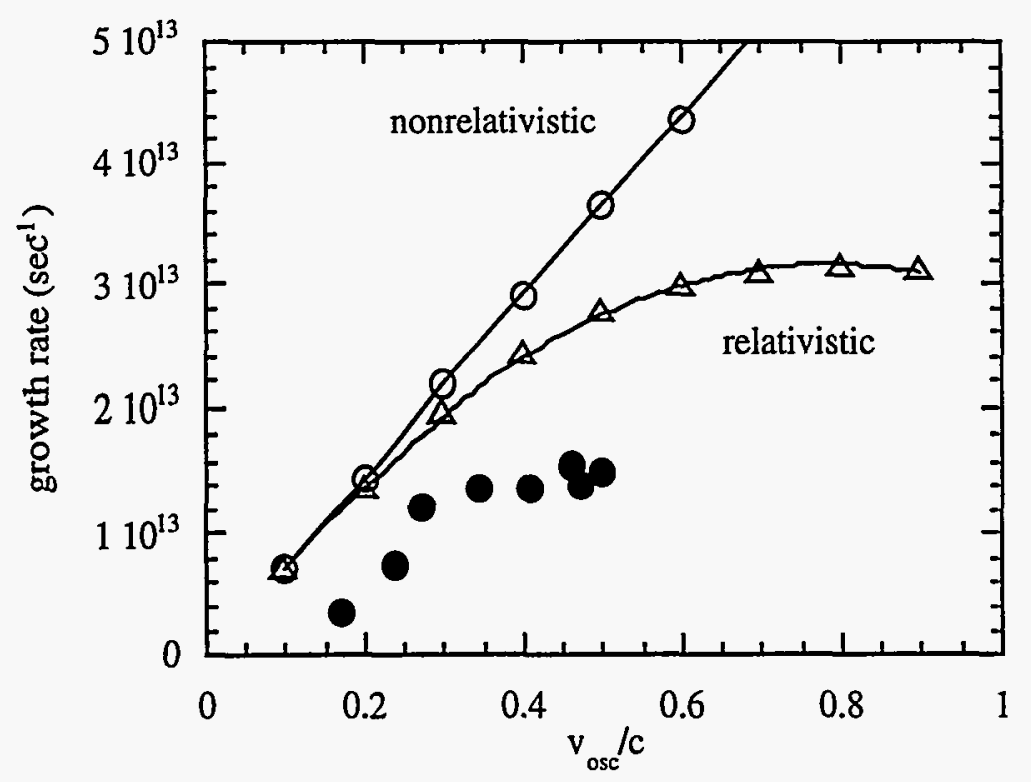

Figure 5.11: Growth rate of the backscattered SRS as a function of intensity. The solid circles represent values of $\gamma_{0}$ extracted from the reflectivity. The open triangles (circles) were calculated using the relativistic (nonrelativistic) growth rate for backscatter.

(2.88), the expression for the relativistic growth rate for backscattered SRS, is also shown in figure 5.11 (open triangles). There is reasonable agreement between the calculated and experimentally measured results (within a factor of 2). The general behavior (shape of the curve) of the experimental growth rate is the same as the relativistic curve. The calculated growth rate is larger than the values extracted from the experimentally measured reflectivity for all the intensitites. The open circles in figure 5.11 show the growth rate obtained using the nonrelativistic growth rate, equation (2.68).

\subsection{Candidate Mechanisms for Modulations}

Several mechanisms have been proposed to explain the modulated stimulated Raman backscatter spectra. Among the most plausible have been self phase modulation, the excitation of a trapped particle instability, randomness in the noise source from 
which the SRS grows, and temporal bursting of the SRS. Each of these mechanisms can generate behavior in a spectrum which resembles some aspect of the behavior observed in the experiments.

One of the most common sources of modulations in high power laser spectra is self phase modulation. Self phase modulation is a self-induced phase shift that can occur when a high power laser propagates through a material with an intensity dependent nonlinear index of refraction. I.e., the index of refraction, $\eta$, can be written as $\eta=\eta_{0}+$ $\eta_{2}$ I. For low intensity, the refractive index is given simply by $\eta_{0}$. For high intensities, however, $\eta_{2} I$ can become significant and alter the index of refraction seen by the propagating laser, resulting in a phase shift. Self phase modulation is the temporal analog to self-focusing, which occurs in the spatial domain as a result of the intensity dependent refractive index. [103] It can appear in a spectrum as a result of ionization of the gas ([95],[127]), the airpath the laser propagates through prior to entering the vacuum chamber, or optics the light propagates through (in this case, in the backscatter path). Self phase modulation can also occur in a plasma via relativistic effects. [78] The index of refraction of a plasma is given by $\eta=\sqrt{1+\omega_{0}^{2} / \gamma \omega_{\mathrm{pe}}{ }^{2}}$, where $\gamma$ is the relativistic correction to the electron mass. In relativistic self phase modulation, the intensity dependent change in the refractive index results from the relativistic correction factor $\gamma$, since $\gamma \sim \sqrt{1+\mathrm{CI}}$, where $\mathrm{C}$ is a constant. This form of self phase modulation is analagous to relativistic self focusing, which has an established threshold given by equation (4.3). This threshold can also be used to determine the regime in which relativistic self phase modulation should occur.

Two simple tests were performed to verify that the modulations of the SRS feature were indeed a plasma effect and not self phase modulation effects. In the first test, the transmitted light was collected and monitored simultaneously with the backscattered light. The transmitted spectrum showed little or no modulation with or without gas in the target chamber. When modulations were observed in the spectrum, they were fewer in number $(\leq 5)$ and not as severe $(\triangle \mathrm{P} / \mathrm{P}<10 \%)$ as those observed in the backscattered spectrum. This indicates that the airpath encountered before the 
beam enters the chamber and the ionization of the gas do not significantly contribute to self phase modulation of the laser pulse. In the second test, a retro-reflecting mirror was placed before the final focus lens and light was propagated along the backscatter path to the spectrometer (see figure 3.1). Approximately 10 times the typical energy associated with the backscattered light was used and no modulations were observed on the collected spectrum. This test further supports the assertion that the modulations in the backscattered SRS feature are indeed a plasma effect and not the result of self phase modulation altering the laser before the interaction region. This does not necessarily rule out the possiblity that phase modulation resulting from propagation of the laser through the constant density plasma (i.e., relativistic self phase modulation [78]) is occuring and inducing modulations in the SRS spectrum. However, for most of the plasma densities and laser intensities for which modulated spectra were observed, the threshold given by equation (4.3) was not surpassed and it is believed that relativistic self phase modulation did not significantly contribute to the observed modulations.

The symmetric, sideband-like behavior of the modulated SRS feature described in section 5.3.1 is suggestive of a trapped-particle-like instability mechanism. In this process, the wave amplitude (the electron plasma wave) becomes large enough to trap plasma electrons in the troughs of the wave. These trapped electrons oscillate in the "wells" at a characteristic frequency, $\omega_{\mathrm{b}}$. ([66], [67])

$$
\omega_{\mathrm{b}}=\omega_{\mathrm{pe}} \sqrt{\frac{\delta \mathrm{n}}{\mathrm{n}}}
$$

where

$$
\frac{\delta \mathrm{n}}{\mathrm{n}}=\frac{\mathrm{ekE}}{\mathrm{m} \omega_{\mathrm{pe}}{ }^{2}}
$$

This time-dependent perturbation to the electron density modulates the current which drives the backscattered SRS (which is oscillating at $\omega_{\mathrm{bs}}$ ), leading to spectral sidebands separated by $\omega_{\mathrm{b}}$. I.e.,

$$
\left(\omega_{\mathrm{bs}}\right)^{\prime}=\omega_{\mathrm{bs}} \pm \mathrm{m} \omega_{\mathrm{b}}
$$

where $m \geq 1$. This would appear in the spectrum as regularly spaced modulation 
sidebands $\left(\Delta \omega=\omega_{\mathrm{b}}\right)$. In the experimental data, a single period could not be extracted from the modulations of the SRS feature. It is possible for a trapped particle instability to produce more than one characteristic frequency since the electric field of the plasma wave is related to the density perturbation $\delta \mathrm{n} / \mathrm{n}$. For example, if $\delta \mathrm{n} / \mathrm{n}=10^{-4}$ in one region of the excitation region of the plasma and $\delta \mathrm{n} / \mathrm{n}=10^{-2}$ in another, the bounce frequency normalized to the plasma frequency, $\omega_{\mathrm{b}} / \omega_{\mathrm{pe}}$, would be $10^{-2}$ and $10^{-1}$, respectively, giving two different periods. Particles with different energies will be trapped at different 'depths' in the trough and oscillate with correspondingly varying degrees of nonlinearity. Therefore, more deeply trapped electrons would execute anaharmonic phase space orbits leading to harmonic spectral components of the bounce frequency $\left(\omega_{\mathrm{b}}, 2 \omega_{\mathrm{b}}, 3 \omega_{\mathrm{b}}\right.$, etc.).

In the experiments, $\delta \mathrm{n} / \mathrm{n}$ ranged from $5 \times 10^{-4}$ at low intensity to $2 \times 10^{-2}$ at high intensity for $\mathrm{n}_{0}=3 \times 10^{17} \mathrm{~cm}^{-3}$ (see figure 5.10). These values of $\delta \mathrm{n} / \mathrm{n}$ would give bounce frequencies of $0.022 \omega_{\mathrm{pe}}$ and $0.14 \omega_{\mathrm{pe}}$, respectively. The separation between individual modulation peaks ranged from approximately $0.033 \omega_{\mathrm{pe}}$ to $0.1 \omega_{\mathrm{pe}}$ in these spectra. Clearly, if a trapped particle instability were present, the bounce frequency associated with the density fluctuation could produce modulations with separations similar to those experimentally observed. For the parameters of these experiments, however, $\omega_{\mathrm{b}} \tau_{\text {laser }} \leq 1$. This implies that fewer than one 'bounce' could occur within the temporal duration of the laser pulse. Therefore, $\omega_{\mathrm{b}}$-modulations of the SRS feature could not contribute to the observed spectra on a relevant timescale.

A third candidate mechanism for the modulated stimulated Raman backscatter feature involves the noise source from which the SRS instability grows. If the noise source is assumed to be spatially smooth and uniform (smoothly varying), one would expect a smooth, uniform spectrum. A noise source with some random spatial structure, however, would result in a modulated spectrum since the SRS feature is effectively an amplification of the noise source (see sections 2.2 and 2.3.1). For a random noise source to be responsible for the modulations observed in these experiments, the randomness would have to be intensity dependent since the degree of modulation is 
observed to increase with increasing intensity. Ion acoustic turbulence $\left(\gamma_{\text {sbs }} \tau \geq 3\right.$ for the higher intensities of these experiments, where $\gamma_{\text {sbs }}$ is the weakly coupled growth rate for stimulated Brillouin scatter) is one effect which could affect the randomness of the noise source. Since the level of turbulence, if present, would vary with the incident laser intensity, this could produce an intensity-dependent source. While a random noise source can produce a severly modulated SRS spectrum (see figure 2.8, for example), it would not necessarily give symmetric structure in the modulations, however.

While each of the above mechanisms can produce behavior similar to that observed in the experiments, none of them individually can account for all the characteristics observed in the experimental spectra. Therefore, the most plausible cause of the observed spectral features is temporal bursting of the stimulated Raman backscatter ([23],[83]). Temporal bursting can produce spectral modulations because the corresponding spectral signature will consist of amplitude modulation (AM) like features (i.e., symmetric structure around a central wavelength). This is illustrated by a simple example in figure 5.12. Figure 5.12(a) shows a series of Gaussian wavepackets with a constant temporal separation. The Fourier transform of the sum of these wavepackets produces a power spectrum, shown in figure 5.12(b). Clearly, the spectrum is modulated, with a distinct symmetry pattern present relative to the central peak. The modulations in this case are separated by a constant frequency. If the wavepackets have random temporal separations, the frequency separations of the modulations in the spectra is no longer ordered, as illustrated in figure 5.12(d). The temporal separation of the individual wavepackets for this spectrum is shown in figure 5.12(c). While the separation between adjacent modulations is no longer constant, a distinct symmetric structure is still apparent.

Recent one-dimensional relativistic particle-in-cell simulations performed at UCLA and at the General Physics Institue in Moscow show evidence of temporal bursting of the scattered light wave associated with SRS. ([23],[83]) These simulations were 

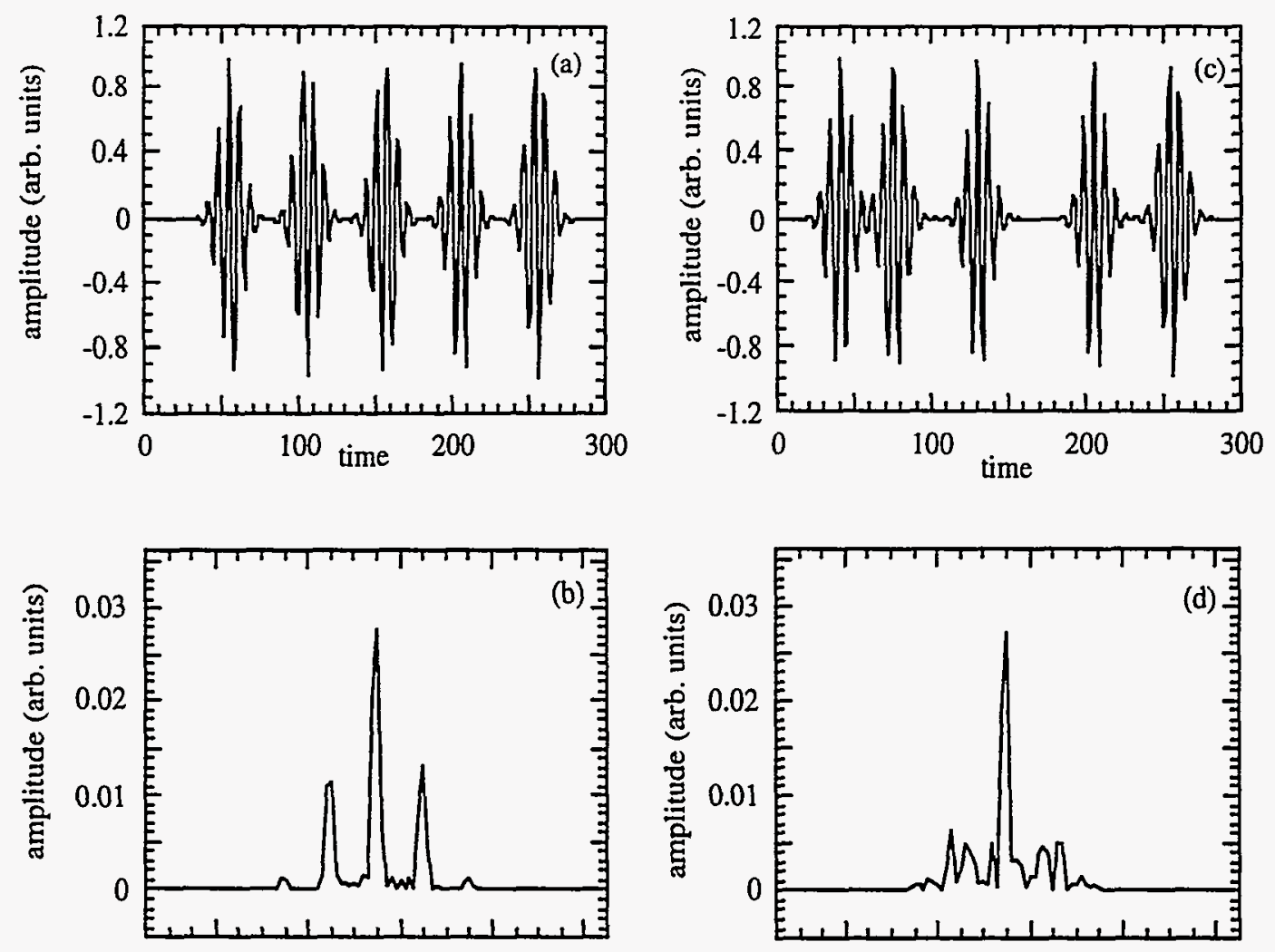

Figure 5.12: Gaussian wavepackets and their corresponding spectra for constant temporal separation of the wavepackets $((a)$ and $(b))$ and random temporal wavepacket separations ((c) and (d)).

performed for parameters similar to those of the experiments in which the modulated SRS spectra were observed. I.e., $3 \times 10^{17} \mathrm{~cm}^{-3} \leq \mathrm{n} \leq 3 \times 10^{19} \mathrm{~cm}^{-3}$ and $\mathrm{v}_{\mathrm{osc}} / \mathrm{c}=0.1-1$. [30] From the preceding discussion, it appears likely that the cause of the modulations is temporal bursting. However, the mechanism responsible for this bursting has not yet been identified.

\subsection{Anomalous Features at Higher Densities}

The observations of backscattered SRS described thus far in this chapter were all obtained at plasma densities below $2 \times 10^{18} \mathrm{~cm}^{-3}$ (static fill experiments). At higher plasma densities, $\left(\mathrm{n}_{0} \geq 5 \times 10^{18} \mathrm{~cm}^{-3}\right.$, gas jet experiments), similar behavior of the 
SRS feature is observed. The SRS feature broadens and modulations begin to appear as the incident laser intensity increases, resulting in, among other things, difficult to interpret density data in cases when backscattered SRS is used as a density diagnostic. At these densities, however, another anomalous feaure appears in the backscattered

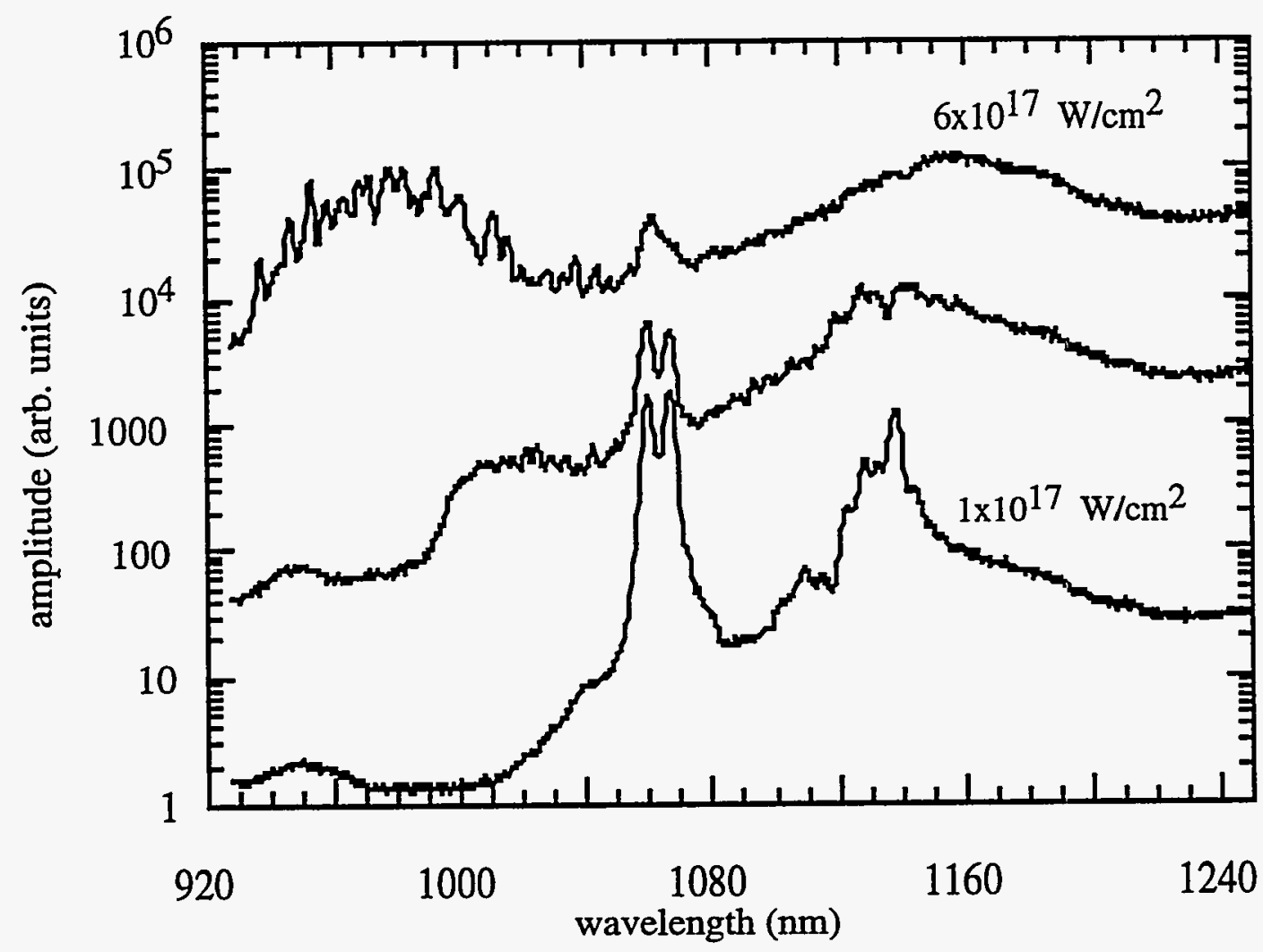

Figure 5.13: Evolution of the backscattered spectrum for a fixed plasma density of $\mathrm{n}_{0}=8 \times 10^{18} \mathrm{~cm}^{-3}$. The laser intensity ranged from $1 \times 10^{17} \mathrm{~W} / \mathrm{cm}^{2}$ to $6 \times 10^{17} \mathrm{~W} / \mathrm{cm}^{2}$.

spectrum. The evolution of the backscattered spectrum for high density is shown in figure 5.13. These spectra have been corrected for the sensitivity of the CCD camera over this wavelength range. This correction procedure, which involves division of the spectrum by the measured response of the $C C D$, leads to the observed sloped spectral baseline. In this figure, the plasma density was fixed at $8 \times 10^{18} \mathrm{~cm}^{-3}(1000$ psi gas jet backing pressure). The laser intensity ranged from $\mathrm{v}_{\mathrm{osc}} / \mathrm{c}=0.29$ to 0.7 .

At low intensity $\left(\mathrm{v}_{\mathrm{osc}} / \mathrm{c}=0.29\right)$, there is a distinct $\mathrm{SRS}$ feature red shifted from 
the laser fiducial (peak in center of plot). As the intensity increases, a clear feature blue-shifted with respect to the laser wavelength begins to appear and the red-shifted SRS broadens. Further increases in intensity show that the blue and red features contribute equally to the total scattered energy. The CCD camera used to record the spectrum is much more sensitive to wavelengths associated with this blue-shifted feature than it is to the red-shifted SRS (approximately 10 times more sensitive). As mentioned earlier, the spectra shown in figure 5.13 have been corrected for this sensitivity.

The energy content of the red and blue spectral components of the backscatter were measured as a function of incident laser intensity in the same manner that reflectivity was measured in section 5.3.3. In this case, however, the "red reflectivity" was obtained by integrating the area to the red side of the laser wavelength $(1052.7 \mathrm{~nm})$ and the "blue reflectivity" was obtained by integrating over the area on the blue side of the laser wavelength. These reflectivities are shown in figure 5.14, where the redshifted (open circles) and blue-shifted (solid circles) features are shown as a function of incident laser intensity. The plasma density, $\mathrm{n}_{0}$, was fixed at $8 \times 10^{18} \mathrm{~cm}^{-3}(1000$ psi helium backing pressure). Both the red-feature and blue-feature reflectivity values initially increased with increasing intensity. The red reflectivity (SRS reflectivity) saturated for intensities above $v_{o s c}>0.4$, as observed in reflectivity measurements made with lower plasma densities. The reflectivity of the blue feature continued to increase above $v_{o s c} / c=0.4$, appearing to saturate near $v_{\text {osc }} / c \approx 0.7$. The red-shifted feature saturated at approximately $10^{-4}$, while the blue-shifted feature had a peak reflectivity near $2 \%$. These values were obtained with a calibration factor generated using the incident laser light (see section 5.3.3). This calibration underestimates the energy for red-shifted features (by approximately a factor of 5) and overestimates the energy associated with blue-shifted features (by approximately a factor of 10) due to the sensitivity of the CCD. The absolute reflectivity, therefore, may not be completely numerically accurate since these values were calculated using spectra which were not corrected for the CCD sensitivity. Error bars have been placed on the reflectivities 


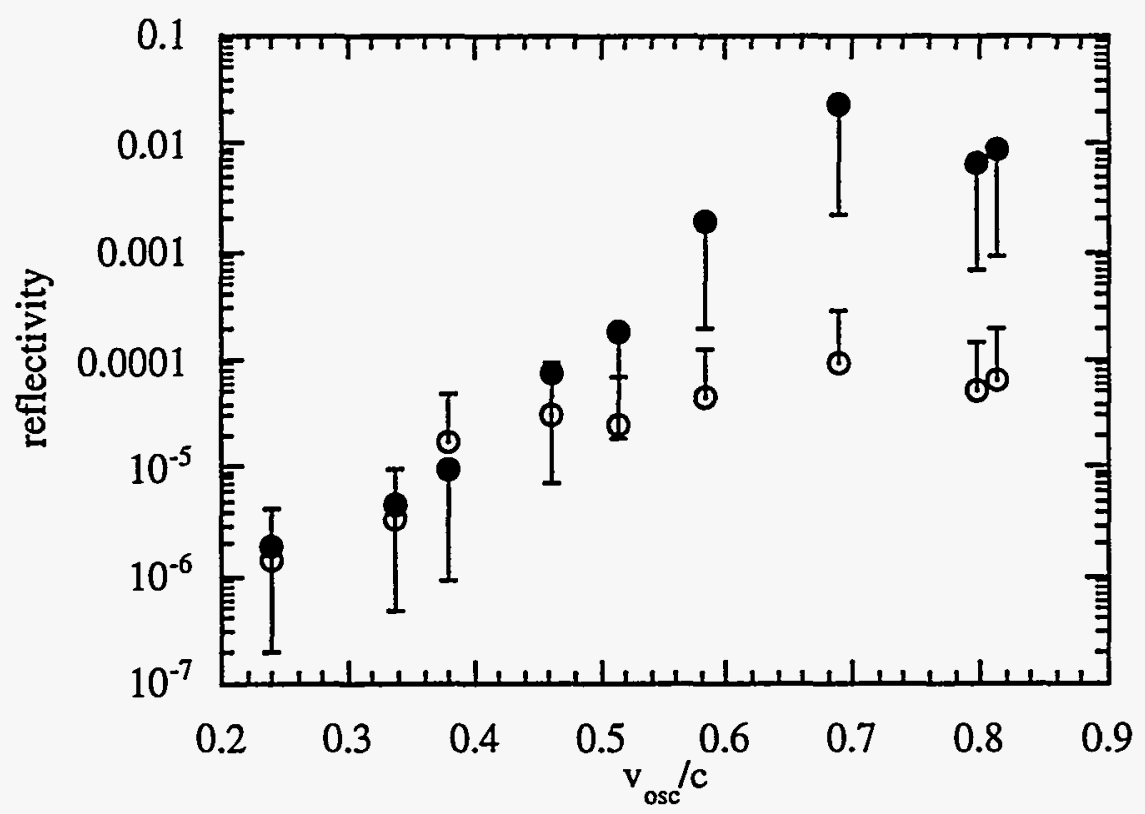

Figure 5.14: Reflectivity of the red-shifted (open circles) and blue-shifted (solid circles) features in the backscattered spectrum. The plasma density was fixed at $8 \times 10^{18} \mathrm{~cm}^{-3}$.

shown in figure 5.14 to illustrate this uncertainty. These error bars indicate that the measured reflectivies for the red-shifted and blue-shifted features are similar.

This distinct blue-shifted feature may result from a coupling between the plasma wave of forward stimulated Raman scatter $\left(\omega_{\mathrm{p}}, \mathrm{k}_{\mathrm{p}}\right)$ and the ion acoustic wave of stimulated Brillouin backscatter $\left(\omega_{\mathrm{ia}}, \mathrm{k}_{\mathbf{i} \mathbf{z}}\right)$. ([36],[59],[69],[50]) These two waves, both of which are forward going, give $\left(\omega_{\mathrm{p}} \pm \omega_{\mathrm{ia}}, \mathrm{k}_{\mathrm{p}} \pm \mathrm{k}_{\mathrm{ja}}\right)$ when coupled. A scattered light wave is produced when the coupled wave interacts with the incident light wave. This scattered light wave is given by $\left(\omega_{0} \pm\left(\omega_{\mathrm{p}} \pm \omega_{\mathrm{ia}}\right), \mathrm{k}_{0} \pm\left(\mathrm{k}_{\mathrm{p}} \pm \mathrm{k}_{\mathrm{ia}}\right)\right)$. The frequency of the plasma wave, $\omega_{\mathrm{p}}$, is approximately the plasma frequency, $\omega_{\mathrm{pe}}$, since the plasma is assumed to be cold. The frequency associated with the ion acoustic wave, $\omega_{\mathrm{ia}}$, is much smaller than the plasma frequency, $\omega_{\mathrm{pe}}$ while the wavenumber, $\mathrm{k}_{\mathrm{ia}}$, is given by $2 k_{0}$ (SBS is backscattered). The wavenumber of the electron plasma wave, $k_{p}$ is much smaller than $k_{0}$ (since forward SRS). 
Two of the scattered waves are given by $\left(\omega_{0}+\omega_{\mathrm{pe}}, 3 \mathrm{k}_{0}\right)$, a blue-shift relative to $\omega_{0}$, or $\left(\omega_{0}-\omega_{\mathrm{pe}}, 3 \mathrm{k}_{0}\right)$, a red shift relative to $\omega_{0}$, where $\omega_{\mathrm{ia}}$ and $\mathrm{k}_{\mathrm{p}}$ have been neglected. These waves do not propagate. The backscattered wave is either a blue-shift, $\left(\omega_{0}+\right.$ $\left.\omega_{\mathrm{pe}},-\mathrm{k}_{0}\right)$ or a red-shift $\left(\omega_{0}-\omega_{\mathrm{pe}},-\mathrm{k}_{0}\right)$ relative to the incident laser frequency. In the backscattered spectrum, the red-shift would appear as an enhancement of stimulated Raman backscatter whereas the blue-shift would be located near where the anti-Stokes SRS feature would be expected (if it were resonant).

For coupling between the forward SRS plasma wave and SBS ion acoustic wave to occur, both forward SRS and SBS must first be driven in the plasma. The regime in which these instabilities can be observed in a plasma (i.e., $\gamma_{0} \tau \geq 1.4$ ) is shown in

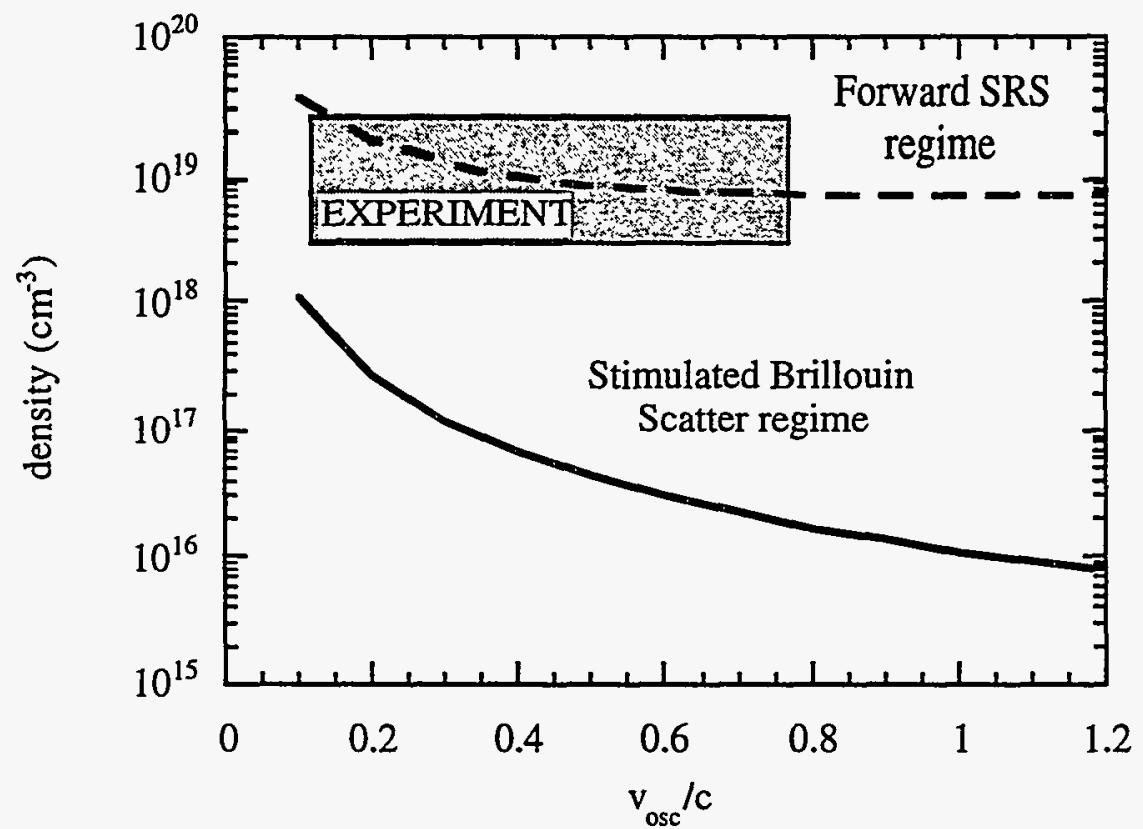

Figure 5.15: Density and intensity regime for which SBS and forward SRS both grow sufficiently to couple. The experimentally accessible region is also shown.

figure 5.15. The experimentally accessible region is also depicted. The density regime in which forward SRS is driven lies well above that required for SBS. Clearly, for all the densities and intensities where stimulated Raman forward scatter has appreciable growth, stimulated Brillouin backscatter is also present. Therefore, the coupling 
described earlier appears to be a plausible mechanism for the anomalous blue feature observed in the backscatter experiments. Spectrally, one would expect to see evidence of not only this coupling, but also SBS in the backscattered spectrum, as well as forward SRS features in the forward scattered spectrum.

The parameters for which the anomalous blue feature appears in the experiment are similar to those for which forward SRS was observed in the near-forward scattered spectrum (described in section 4.2). Specifically, for intensities above $\mathrm{v}_{\mathrm{osc}} / \mathrm{c}=0.4$, both forward SRS and the anomalous blue-shifted feature in the backscatter were observed. Stimulated Brillouin backscatter was not resolved in the experimental spectra. At best, however, for most of the parameters studied here, the SBS would show up as only a shoulder on the red side of the laser fiducial due to the large bandwidth of the laser (assuming weakly coupled SBS).

If the blue-shifted feature resulted from coupling between the plasma wave and ion acoustic wave, it would be located near $\omega_{0}+\omega_{\text {pe }}$. Therefore, the density of the plasma could be inferred from this feature. Figure 5.16 shows the density inferred from both the red-shifted backscattered SRS (solid circles) and the blue-shifted anomalous feature (open triangles) for several backing pressures of the gas jet. There is a substantial amount of shot-to-shot fluctuation, resulting from the irreproducibility of the gas jet burst, but clearly the densities extracted from the blue feature agree with those obtained from the backscattered SRS.

A second explanation postulated for the anomalous blue feature is stimulated Compton scattering. [126] Stimulated Compton scattering (SCS) is the low-density or high-temperature (large $\mathrm{k} \lambda_{\text {debye }}$ ) limit of stimulated Raman scattering. In the SRS process, the pump electromagnetic wave scatters off an electron plasma wave. For SCS, the pump and scattered light waves interact with the resonant thermal plasma electrons since the electron plasma wave is heavily Landau damped. [71] The growth rate for SCS is much less than that of SRS, unless the plasma wave is heavily damped.

In a recent experiment at UCLA [49], the evolution of SRS into SCS was observed as an initially cold plasma was heated via wavebreaking. Recent 1-dimensional PIC 


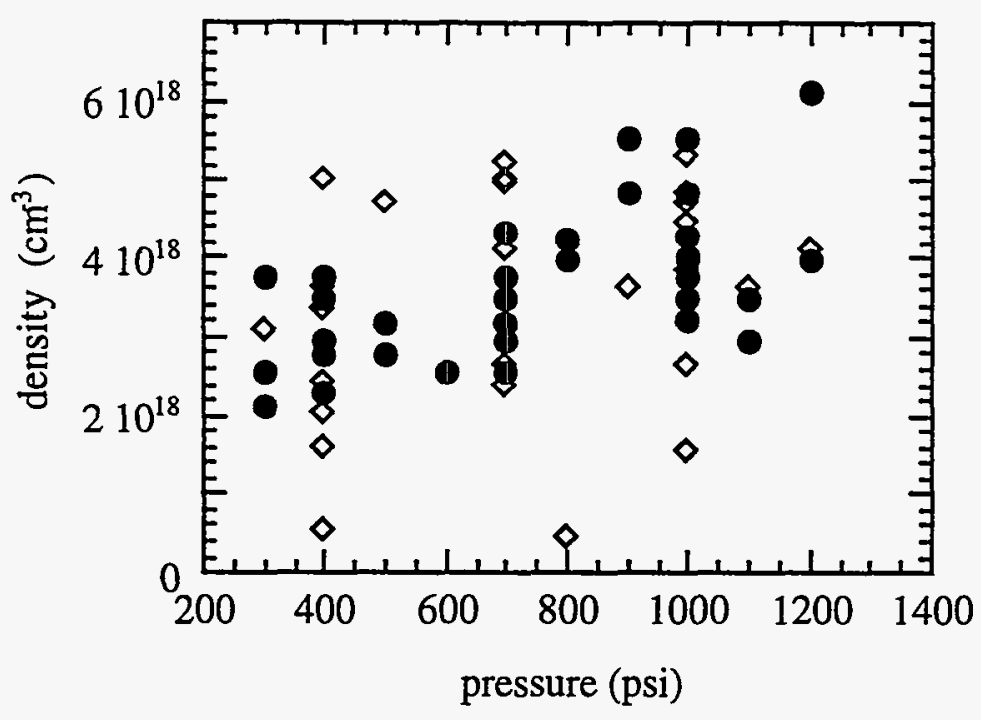

Figure 5.16: Density values inferred from the red-shifted SRS (solid circles) and the anomalous blue feature (open triangles).

simulations (ZOHAR) [126] have shown that significant heating of the plasma occurs for the densities and intensities of these experiments. For example, up to $50 \%$ of the electrons are heated to $2 \mathrm{keV}$, with the remaining electrons near $100 \mathrm{eV}$ for parameters similar to those of the experiments $\left(\mathrm{n} \sim 1 \times 10^{19} \mathrm{~cm}^{-3}, 0.1<\mathrm{v}_{\text {osc }} / \mathrm{c}<1\right)$. These temperatures correspond to $\mathrm{k} \lambda_{\text {debye }}$ values of 0.28 and 1.25 , respectively. Clearly, $\mathrm{k} \lambda_{\text {debye }}$ is large enough $(\geq 0.3)$ at the higher temperature to be in the stimulated Compton regime. In fact, these PIC simulations show evidence of both SRS (from the low temperature plasma) and SCS (from the high temperature plasma). For low $v_{\text {osc }} / c$, only the SRS feature is present. It is narrow, and located near $\omega_{0}-\omega_{\text {pe }}$, as expected. As the laser intensity is increased $\left(n=0.01 n_{c}, v_{o s c} / c=0.4\right)$, a stimulated Compton scatter feature appears in the spectrum as well, indicating that part of the plasma has been heated. This SCS feature is much broader ( $\Delta \omega \approx 0.6 \omega_{0}$ at FWHM) than the SRS feature (which is still present, located at $\omega_{0}-\omega_{\mathrm{pe}}$ ), is of lower absolute amplitude, and has two distinct 'bumps', symmetrically located near $\pm 2 \omega_{\mathrm{pe}}$ relative to the SRS feature.[126] I.e., the two SCS features are located near $\omega_{0}+\omega_{\text {pe }}$ (a blueshifted feature) and $\omega_{0}-3 \omega_{\mathrm{pe}}$ (a second red-shifted feature). The broad SCS feature 
should be located at $\omega_{\mathrm{s}} \approx \omega_{0}-\mathrm{kv}_{\mathrm{th}}$. For this particular case, this corresponds to the location of the SRS feature (i.e., $\mathrm{kv}_{t h} \sim \omega_{\mathrm{pe}}$ ). Further increases in intensity result in further heating of the plasma, shifting the SCS feature farther to the red, away from the SRS feature, and only a single SCS feature is present in the spectrum. [126]

Comparison of the experimentally observed spectra (i.e., figure 5.13) with the simulations suggest that SCS may be occuring in the high intensity spectra as well as SRS. The observed blue feature in the spectrum shifts with increasing intensity, and is located near $\omega_{0}+\omega_{\mathrm{pe}}$ (see figure 5.16). These results are consistent with simulation results obtained for moderate laser intensities, where $\mathrm{kv}_{\mathrm{th}} \sim \omega_{\mathrm{pe}}$. The red-shifted SCS feature (near $\omega_{0}-3 \omega_{p e}$ ) was not observed in the experimental spectra, but this is likely due to experimental limitations since the silicon-based CCD used to collect the spectra is not sensitive to wavelengths above $1100 \mathrm{~nm}$ and the red-shifted SCS feature would be located near $1300 \mathrm{~nm}$. Other experiments performed on the same laser system used in these experiments with similar parameters have shown evidence of the heating of the plasma predicted by the simulations [33], reinforcing the argument that stimulated Compton scattering is responsible for the blue-shifted feature.

\subsection{Summary}

In stimulated Raman backscatter experiments using a short, intense laser pulse interacting with an underdense plasma, several interesting features were observed. The SRS spectra broadened and became severly modulated as both density and intensity were increased. A broad feature underlying these spectra qualitatively agreed with spectra obtained with the dispersion relation, with the exception being the location of the SRS feature. The experimentally observed spectra, while still red-shifted with respect to the laser frequency, were blue-shifted relative to the expected $\omega_{\text {pe }}$ shift. This discrepancy was also seen to increase with intensity. The modulations present on the spectra were narrow, and closely spaced. They often exhibited a high degree of spectral symmetry, but the locations of individual peaks were not reproducible. The 
reflectivity of the red SRS feature increased with increasing intensity, and saturated for intensities above $v_{o s c} / c>0.4$, indicating nonlinear effects may be present in the plasma. Growth rates extracted from this reflectivity were in good agreement with relativistically calculated growth rates for backscattered SRS. Furthermore, the saturation of the reflectivity occured for intensities similar to those for which modulations appeared in the spectra.

Several mechanisms have been postulated for the structure present in the SRS spectra, including self-phase modulation, trapped particles, random noise sources, and temporal bursting of SRS. While each of these mechanisms has merit, it has been demonstrated that three of these suggestions are probably not responsible for the modulations. Simulations suggest that the mechanism for the modulations is temporal bursting of the SRS, but the cause of the bursting is not known.

An anomalous feature blue-shifted from the laser frequency was observed in backscattered spectra obtained with high plasma densities. Mechanisms postulated for this blue feature include coupling between the plasma wave associated with forward SRS and the ion acoustic wave associated with stimulated Brillouin scattering and stimulated Compton scattering. 


\section{Chapter 6}

\section{Summary}

Advances in laser technology in recent years have resulted in compact, terawatt class laser systems. These lasers, capable of generating subpicosecond, intense ( $\geq$ $\left.5 \times 10^{16} \mathrm{~W} / \mathrm{cm}^{2}\right)$ pulses, have provided access to a new parameter regime in which laser-plasma interactions can be studied. In this regime, $\mathrm{L}_{\text {plasma }} \geq 2 \mathrm{~L}_{\text {Rayleigh }}>\mathrm{c} \tau$, where $L_{\text {plasma }}$ is the size of the plasma, $L_{\text {Rayleigh }}$ is the Rayleigh range, and $c \tau$ is the pulse length of the laser. Prior to the development of short pulse lasers, experiments investigating laser-plasma interactions were constrained to the regime defined by $c \tau>\mathrm{L}_{\text {plasma }} \geq \mathrm{L}_{\text {Rayleigh. }}$. In this dissertation, the interaction of a short pulse, high intensity laser with an underdense plasma was studied. Specifically, the parametric instability known as stimulated Raman scatter was experimentally investigated in this new parameter regime. This instability is interesting not only from a basic physics standpoint, but also for its significance to applications such as the fast ignitor concept and $\mathrm{x}$-ray lasers.

The behavior of stimulated Raman scatter in this relatively new parameter regime was predicted with theoretical models based on the coupled partial differential equations and the dispersion relation. The coupled equations, both derived and solved in this work, illustrated the growth and evolution of the electron plasma and scattered light waves which are generated when SRS is driven in an underdense plasma. The dispersion relation, derived from the coupled partial differential equations, was used 
to predict the actual electromagnetic spectrum expected for both stimulated Raman backscatter and stimulated Raman forward scatter. Both the relativistic and nonrelativistic versions of the dispersion relation were solved and the results compared to determine when the relativistic correction factor became significant. The growth rate of the scattered light wave was also modeled, as was the influence of random versus smooth noise sources.

Experiments designed to study stimulated Raman backscatter and forward scatter spectrally were performed. The energetic electrons commonly associated with forward Raman scattering were also investigated. A Nd:glass laser system which produces 600 fsec pulses with intensities up to $10^{18} \mathrm{~W} / \mathrm{cm}^{2}$ at a wavelength of $1.0527 \mu \mathrm{m}$ was used in these experiments. Helium plasma densities ranged from $\mathrm{n}_{0}=1 \times 10^{17} \mathrm{~cm}^{-3}$ to $3 \times 10^{19} \mathrm{~cm}^{-3}$. For densities below $2 \times 10^{18} \mathrm{~cm}^{-3}$, a static fill of helium was used; a high mach number gas jet, backed with helium, was used for experiments with densities higher than $2 \times 10^{18} \mathrm{~cm}^{-3}$.

Near-forward scattered light, when collected and spectrally resolved, clearly showed two of the anti-Stokes features commonly associated with forward stimulated Raman scatter. The location of the first anti-Stokes feature agreed well with the prediction obtained from the dispersion relation. Energetic electrons were observed for the same laser and plasma parameters as the anti-Stokes modes. In fact, there was a distinct correlation between the generation of the spectral features of forward Raman scatter and $2 \mathrm{MeV}$ electrons, clear evidence that stimulated Raman forward scatter was being driven in the underdense plasma. This is the first observation of this instability with a subpicosecond laser.

There was also a distinct correlation between the observance of forward SRS antiStokes modes and a decrease in the level of energy transmitted through the plasma within the original focal cone. At high laser intensities, only $55 \%$ of the incident laser energy was transmitted through the plasma within the focal cone. The correlation between the forward SRS and reduction in transmitted energy suggested that the actual propagation of the short, intense laser pulse through the underdense plasma was 
altered by the generation of the forward Raman instability. This observation agreed quantitatively with results of massively parallel particle in cell computer simulations performed by UCLA for the parameters of the experiments. These same simulations showed that forward SRS leads to longitudinal break-up of the laser beam. The experimental forward SRS observations have raised questions about the stability of short pulse laser propagation through underdense plasmas.

Stimulated Raman backscatter was also observed in these experiments. At low incident laser intensities, a clean SRS feature was observed which agreed with the predictions of the dispersion relation. As the intensity was increased, however, the backscattered spectral feature broadened and developed severe modulations. There was also a shift of spectral energy toward the blue relative to the expected location of the SRS. The general nature of the SRS agreed with dispersion relation predictions, in both shape and width. The appearance of modulations and blue-shifting, however, were not predicted. The modulations in the backscattered spectrum were narrow, closely spaced, and exhibited a distinct symmetric structure. Potential mechanisms for the modulations include the presence of self phase modulation, a trapped particle instability, a random noise source, and temporal bursting of the SRS. Relativistic 1dimensional PIC simulations indicate that temporal bursting produces the modulated SRS, although the mechanism for the bursting is not yet known.

Reflectivity measurements of the modulated backscattered SRS showed a saturation of the instability, implying that nonlinear effects are occuring in the plasma. Growth rates extracted from the reflectivity were consistent in amplitude (within a factor of 2) and general behavior with the relativistically correct predicted growth rate. The peak reflectivity of the SRS (for $\mathrm{n}_{0}=7 \times 10^{17} \mathrm{~cm}^{-3}$ ) was $2 \times 10^{-4}$, indicating that only a small fraction of the incident laser energy was lost in driving backscattered SRS. It is worth noting that the intensity for which the reflectivity saturates $\left(\mathrm{v}_{\text {osc }} / \mathrm{c} \geq 0.35\right)$ is approximately the same intensity at which modulations appear in the spectra.

At higher densities $\left(\mathrm{n}_{0} \geq 5 \times 10^{18} \mathrm{~cm}^{-3}\right)$, a distinct feature appeared in the 
backscattered spectrum, blue-shifted from the laser frequency. This feature may be the result of coupling between the electron plasma wave from forward SRS and the ion acoustic wave associated with stimulated Brillouin scatter or stimulated Compton scatter. Reflectivity measurements of this blue feature show saturation behavior similar to that observed in the backscattered SRS reflectivity.

In conclusion, several new and interesting observations were made in the short, intense laser pulses, underdense plasma experiments of this work. Stimulated Raman forward scatter was observed for the first time in this parameter regime. Experiments studying stimulated Raman backscatter revealed both familiar and anomalous features in the spectra, some of which still elude explanation. These experiments, which represent some of the first in this regime, have illustrated that while the interactions between lasers and plasmas in the long pulse and short pulse regimes are very similar, they are also distinctly different. I expect that in the coming years, the field of short pulse, high intensity lasers interacting with plasmas will continue to produce interesting and unexpected results. 


\section{Appendix A}

\section{Plasma Images}

The size and shape of the plasmas produced by a high intensity laser pulse can be theoretically predicted and compared to experimentally observed plasmas. In this appendix the theory governing the spatial characteristics of a laser produced plasma is reviewed, with examples restricted to parameters of interest in this work. These results are compared with experimentally obtained plasma images. The energy lost to the creation of the plasma is also discussed.

\section{A.1 Theory}

The intensity distribution for a Gaussian laser pulse is given by

$$
\mathrm{I}(\overrightarrow{\mathrm{r}})=\mathrm{I}_{0}\left(\frac{\mathrm{W}_{0}}{\mathrm{r}_{0}}\right)^{2} \mathrm{e}^{-2 \mathrm{r}^{2} / \mathrm{r}^{2}}
$$

where $\mathbf{r}$ is the radial distance from the laser axis, $I_{0}$ is the peak intensity, $r_{0}=$ $\mathrm{w}_{0}\left(1+\mathrm{z}^{2} / \mathrm{z}_{0}^{2}\right)^{\frac{1}{2}}$ is the radius of the laser spot ("spot size"), $\mathrm{z}_{0}=\pi \mathrm{w}_{0}^{2} / \lambda$ is the Rayleigh length, and $w_{0}$ is the minimum radius of the focal spot. [103] Rearranging the terms of equation (A.1) provides an expression for the radial distance from the laser axis, $\mathrm{r}$, as a function of the intensity, $I$, and position, $\mathrm{z}$.

$$
r(z)=w_{0}\left[\frac{1}{2}\left(1+\frac{z^{2}}{z_{0}^{2}}\right)\left[\ln \left(\frac{I_{0}}{I}\right)-\ln \left(1+\frac{z^{2}}{z_{0}^{2}}\right)\right]\right]^{\frac{1}{2}}
$$


Isointensity contours of the focal region can be determined from this equation, as shown in figure A.1. In this plot, isointensity contours are shown for intensities, I,

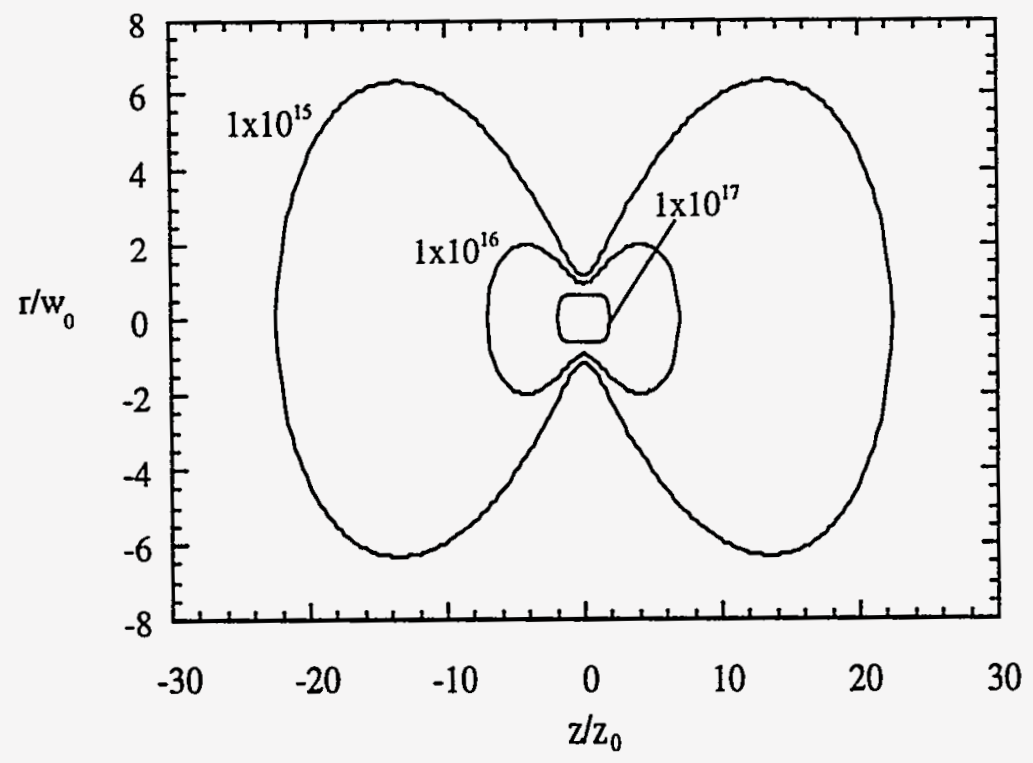

Figure A.1: Isointensity contours for a Gaussian distribution

of $1 \times 10^{15}, 1 \times 10^{16}$, and $1 \times 10^{17} \mathrm{~W} / \mathrm{cm}^{2}$. The peak intensity, $\mathrm{I}_{0}$, was chosen to be $5 \times 10^{17} \mathrm{~W} / \mathrm{cm}^{2}$. The longitudinal distance, $z$, is plotted on the $z$-axis $\left(z / z_{0}\right)$ and the radial distribution, $r$, is plotted on the $y$-axis $\left(r / w_{0}\right)$. For intensities much lower than the peak intensity $\left(I \ll I_{0}\right)$, the isointensity contour has a double lobe shape. At higher intensities, only one central lobe is present. The three dimensional distribution of the intensity can be obtained by rotating the contours about the z-axis. It should be noted that the isointensity curves shown in figure A.1 do not represent the actual aspect ratio of $r$ and $z$ since these quantities have been normalized to $w_{0}$ and $z_{0}$, respectively, and $z_{0}=\pi \mathrm{w}_{0}{ }^{2} / \lambda$.

The maximum value of the axial dimension, $z$, can be obtained from equation (A.2) by letting $r=0$. This value of $z$ represents the spatial extent of the volume for which the intensity is greater than or equal to the value $I$. The maximum value is given by

$$
z_{\max }= \pm z_{0} \sqrt{\frac{I_{0}}{I}-1}
$$


For the isointensity contours shown in figure A.1, the values of $z_{\max } / z_{0}$ are 22,7 , and 2 for $\mathrm{I}=1 \times 10^{15}, 1 \times 10^{16}$, and $1 \times 10^{17} \mathrm{~W} / \mathrm{cm}^{2}$, respectively.

The theoretically expected ionization profile (density distribution) of a plasma produced with a high intensity laser can be determined from equations (A.2) and (A.3). The assumptions employed in this calculation include perfect Gaussian focusing of the laser, no gas-induced or ionization induced refraction, and negligible pump depletion. The validity of these assumptions is discussed further later in this appendix and in section 3.5.1. Many different charge states of an ion may be present, depending on the gas used and the f-number and peak intensity of the laser. When short pulse, high intensity lasers are used to create plasmas from gaseous targets, ionization usually occurs via a process known as tunneling ionization. [5] Tunneling ionization is an ionization process in which the Coulomb barrier of the nucleus of the atom is suppressed by the laser field, allowing an electron to tunnel through the barrier. This regime is defined by $\kappa>1$, where the Keldysh parameter, $\kappa$, is given by $\left(E_{\text {ion }} / 2 \Phi_{\mathrm{p}}\right)^{\frac{1}{2}}, E_{\text {ion }}$ is the ionization potential of a particular charge state of an atom and $\Phi_{\mathrm{p}}$ is the ponderomotive potential of the laser field. [60] For short, intense laser pulses, the laser field is typically on the order of or greater than the atomic field binding the electrons to the nucleus, allowing tunneling ionization to occur.

As an example of how equations (A.2) and (A.3) can be used to predict the ionization profile of a plasma produced from a gaseous target, helium is examined. A threshold intensity of $4 \times 10^{14} \mathrm{~W} / \mathrm{cm}^{2}$ is necessary to generate $\mathrm{He}^{+}$whereas the threshold intensity for $\mathrm{He}^{2+}$ is $2 \times 10^{15} \mathrm{~W} / \mathrm{cm}^{2}$. Above $4 \times 10^{15} \mathrm{~W} / \mathrm{cm}^{2}$, the saturation intensity for $\mathrm{He}^{2+}$, only $\mathrm{He}^{2+}$ will be present. The intensities cited here were experimentally measured with the laser system used in the experiments of this work. [32] Clearly, for a particular peak intensity incident on an infinite region of gas (i.e., static fill, many Rayleigh lengths), there will be an intensity region in which only $\mathrm{He}^{+}$will be present and a smaller volume in which $\mathrm{He}^{2+}$ will exist. The density in the $\mathrm{He}^{2+}$ region will be higher than that of the $\mathrm{He}^{+}$region by a factor of 2 . For a gas of finite extent (gas jet burst, few Rayleigh lengths) and a high peak laser intensity $\left(\geq 5 \times 10^{15} \mathrm{~W} / \mathrm{cm}^{2}\right)$, it 
is possible to generate a uniform plasma where $\mathrm{He}^{2+}$ is the only charge state present in the plasma over a longitudinal distance determined either by $z_{\max }$ (equation (A.3)) or the extent of the gaseous region. The radial uniformity is determined by equation (A.2) for a fixed peak laser intensity. Figure A.2 shows the spatial distributions of $\mathrm{He}^{+}$and $\mathrm{He}^{2+}$ for a peak incident intensity of $\mathrm{I}_{0}=2 \times 10^{17} \mathrm{~W} / \mathrm{cm}^{2}$ and an infinite gaseous region. The shaded area shows the spatial regime where both charge states will be present. The vertical lines isolate the spatial region of interest when a gas of .

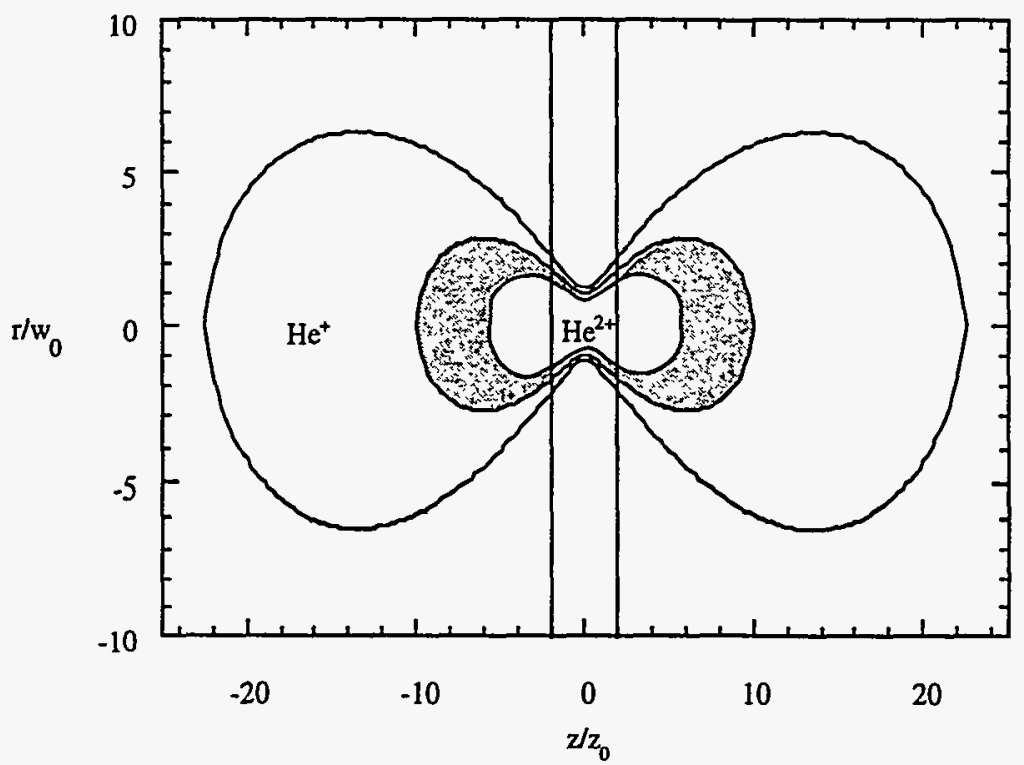

Figure A.2: Spatial distribution for $\mathrm{He}^{+}$and $\mathrm{He}^{2+}$ for a peak laser intensity of $2 \times$ $10^{17} \mathrm{~W} / \mathrm{cm}^{2}$.

finite extent is used (here, $z= \pm 2 z_{0}$ ). In this example, for $|z|>2 z_{0}$, there is no gas to ionize and therefore no plasma present. For a localized helium plasma produced with a high intensity laser, $\mathrm{He}^{2+}$ is the only charge state present (for $\left|\mathrm{r} / \mathrm{w}_{0}\right|<1$ ). For $1<\left|r / w_{0}\right|<2$, the density will decrease by about a factor of 2 over the longitudinal region defined by $\left|z_{0}\right| \leq 2$. There is not sufficient laser intensity to ionize the helium for $\left|r / w_{0}\right|>2$.

The volume associated with an intensity exceeding a particular value (i.e., the volume in which a particular charge state is present) can also be calculated from 
equations (A.2) and (A.3). [92] This volume, $\mathrm{V}$, is given by

$$
\mathrm{V}=\int_{-\mathrm{z}_{\max }}^{z_{\max }} \pi \mathrm{r}(\mathrm{z})^{2} \mathrm{dz}
$$

Substitution of $r(z)$ (equation (A.2)) into this expression, gives, upon integration,

$$
\mathrm{V}=\frac{\pi^{2} \mathrm{~W}_{0}^{4}}{\lambda}\left[\frac{2}{9} \mathrm{~A}^{3}+\frac{4}{3} \mathrm{~A}-\frac{4}{3} \tan ^{-1} \mathrm{~A}\right]
$$

where $A=z_{\max } / z_{0}=\sqrt{I_{0} / I-1}$. For the contours shown in figure A.2, the volume of the $\mathrm{He}^{+}$region is $2 \times 10^{-3} \mathrm{~cm}^{-3}$ while the $\mathrm{He}^{2+}$ region has a volume of $6.5 \times 10^{-5} \mathrm{~cm}^{-3}$. A spot size, $\mathrm{w}_{0}$, of $17 \mu \mathrm{m}$ (see chapter 3 ) was used to calculate these values.

The energy required to ionize a region of gas can be determined once the interaction volume is obtained. This energy is given by

$$
\mathrm{E}_{\mathrm{ion}}=\mathrm{n}_{0} \mathrm{VU}^{\text {charge state }}
$$

where $n_{0}$ is the neutral atom density and $U^{\text {chargestate }}$ is the ionization potential of a particular charge state. This formula includes only tunneling ionization of the gas. This energy represents the pump depletion experienced by the incident laser beam as it ionizes the neutral gas. When the pump is depleted through ionization, less laser energy (intensity) is available to interact with the plasma. For atoms with more than one accessible charge state, the total energy lost is given by

$$
E_{\text {total }}=\sum_{\mathbf{n}} E_{n}
$$

where $E_{n}$ is the energy required to ionize from charge state (n-1) to charge state n. Note that $E_{n}=n_{0} V_{n} U^{n}$ where $V_{n}$ is the volume over which the charge state $\mathrm{n}$ is accessible, calculated from equation (A.5). For helium, $\mathrm{U}^{\mathrm{He}+}=24.59 \mathrm{eV}$ and $\mathrm{U}^{H e^{2+}}=54.41 \mathrm{eV}$. [31] Therefore, for a peak laser intensity of $2 \times 10^{17} \mathrm{~W} / \mathrm{cm}^{2}$ and an infinite volume of helium gas with atom density of $7 \times 10^{17} \mathrm{~cm}^{-3}$, approximately $1 \%$ of the incident intensity is lost when the neutral gas is ionized to $\mathrm{He}^{2+}$. Parameters relevant to the laser system described in chapter 3 were used to obtain this value (i.e., $84 \%$ of the energy is contained within a $13.6 \mu \mathrm{m} \times 20.4 \mu \mathrm{m}$ "spot," $600 \mathrm{fsec}$ 


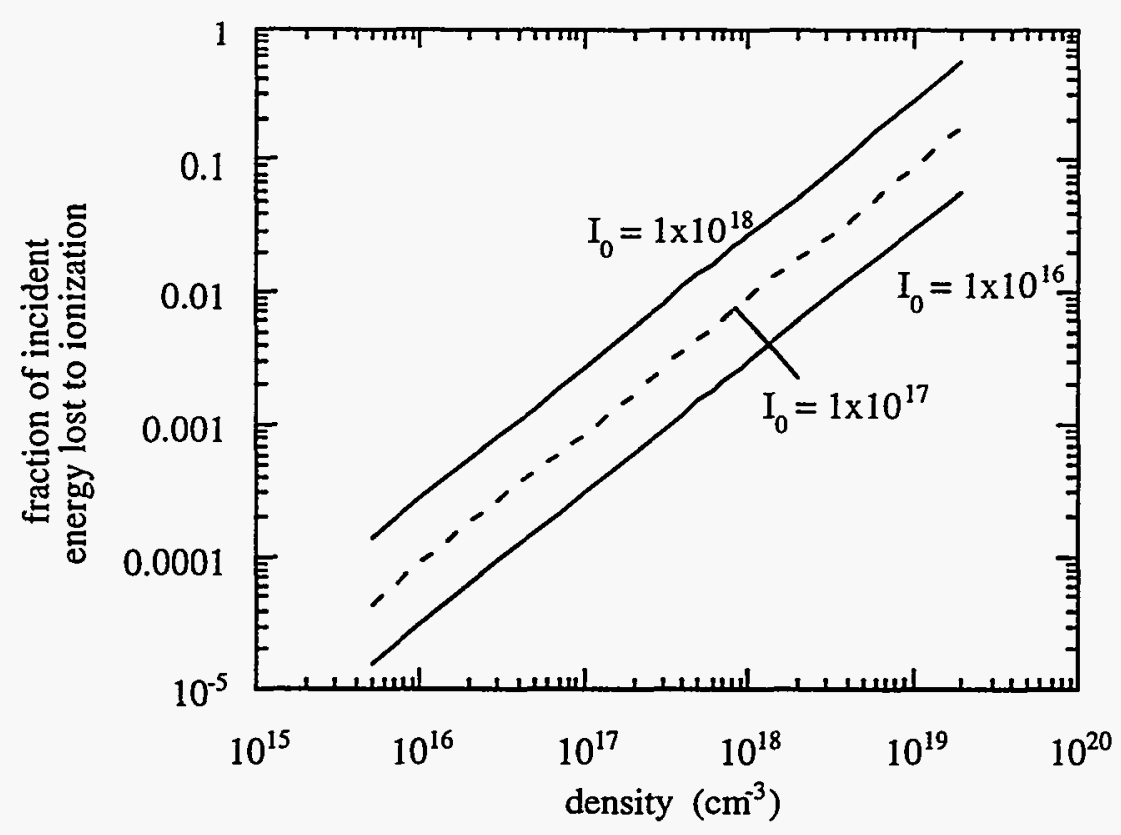

Figure A.3: Fraction of incident energy of a focused Gaussian laser beam lost to ionization as a function of density for an infinite volume of gas.

pulsewidth). Figure A.3 shows the energy lost to ionization of an infinite volume of helium for several densities and intensities. For low densities $\left(\leq 10^{18} \mathrm{~cm}^{-3}\right)$, only a small fraction of a percent of the incident energy is lost to ionization of the helium. Even at the highest intensities obtainable with the $10 \mathrm{TW}$ laser, only a few percent is lost. At higher densities ( $>10^{18} \mathrm{~cm}^{-3}$ ), however, a significant fraction of the incident energy is lost to ionization, particularly at the higher laser intensities. For a density of $1 \times 10^{19} \mathrm{~cm}^{-3}$, almost $10 \%$ of the incident energy is lost to ionization for $\mathrm{I}_{0}=1 \times 10^{17} \mathrm{~W} / \mathrm{cm}^{2}$. In obtaining these values, however, an infinite region of gas was assumed. The plasmas created with high intensity lasers in an infinite region of gas are very long, as shown in figure A.4. In this figure, the length of the plasma is plotted as a function of intensity for the $\mathrm{He}^{+}$region and the $\mathrm{He}^{2+}$ region. The Rayleigh range, $2 z_{0}$, was assumed to be approximately $1 \mathrm{~mm}$, the focal depth measured for the laser used in this work (see section 3.2). At a laser intensity of $1 \times 10^{17} \mathrm{~W} / \mathrm{cm}^{2}$, a $\mathrm{He}^{+}$plasma $2.5 \mathrm{~cm}$ long (along the laser axis) is generated. 


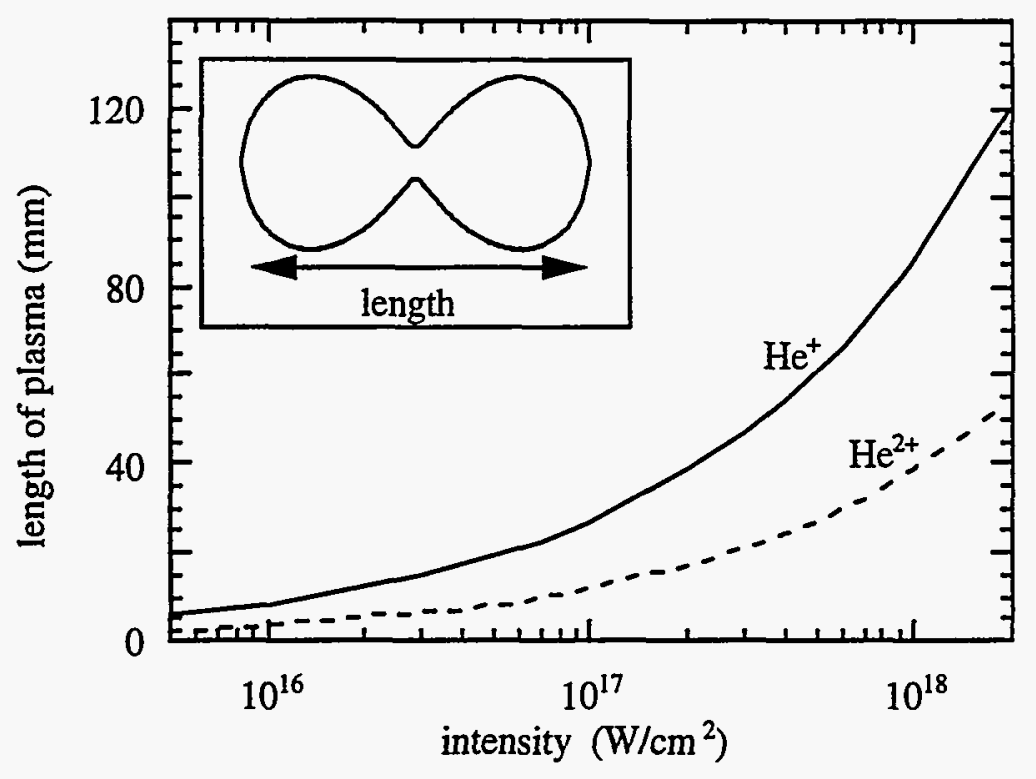

Figure A.4: Length of $\mathrm{He}^{+}$and $\mathrm{He}^{2+}$ plasmas as a function of incident laser intensity.

The $\mathrm{He}^{2+}$ region of plasma is approximately $1 \mathrm{~cm}$ long. Clearly, to generate such large plasmas requires substantial energy. Figure A.3, combined with the ionization induced refraction discussed in section 3.5.1, illustrates the need for a localized region of gas (plasma) when densities greater than $2 \times 10^{18} \mathrm{~cm}^{-3}$ are used with high intensity lasers. If a localized region of gas approximately $1 \mathrm{~mm}$ long (along the laser axis) is used, obtainable with a gas jet for example, the fraction of energy lost to ionization for a density of $1 \times 10^{19} \mathrm{~cm}^{-3}$ and a peak intensity of $1 \times 10^{18} \mathrm{~W} / \mathrm{cm}^{2}$ is only $0.014 \%$.

\section{A.2 Experiments}

Images of experimentally produced plasmas were taken at a variety of plasma densities and laser intensities for comparison with the calculated size and shape of the plasma. The experimental configuration used to obtain the images is shown in figure A.5. The $1.0527 \mu \mathrm{m}$ laser was focused by an aspheric $\mathrm{f} / 8.2$ lens into a vacuum vessel statically filled with helium gas. The plasmas were imaged $90^{\circ}$ relative to the laser axis with an $f / 12$ Nikon lens attached to a liquid nitrogen cooled CCD camera. Narrowband 


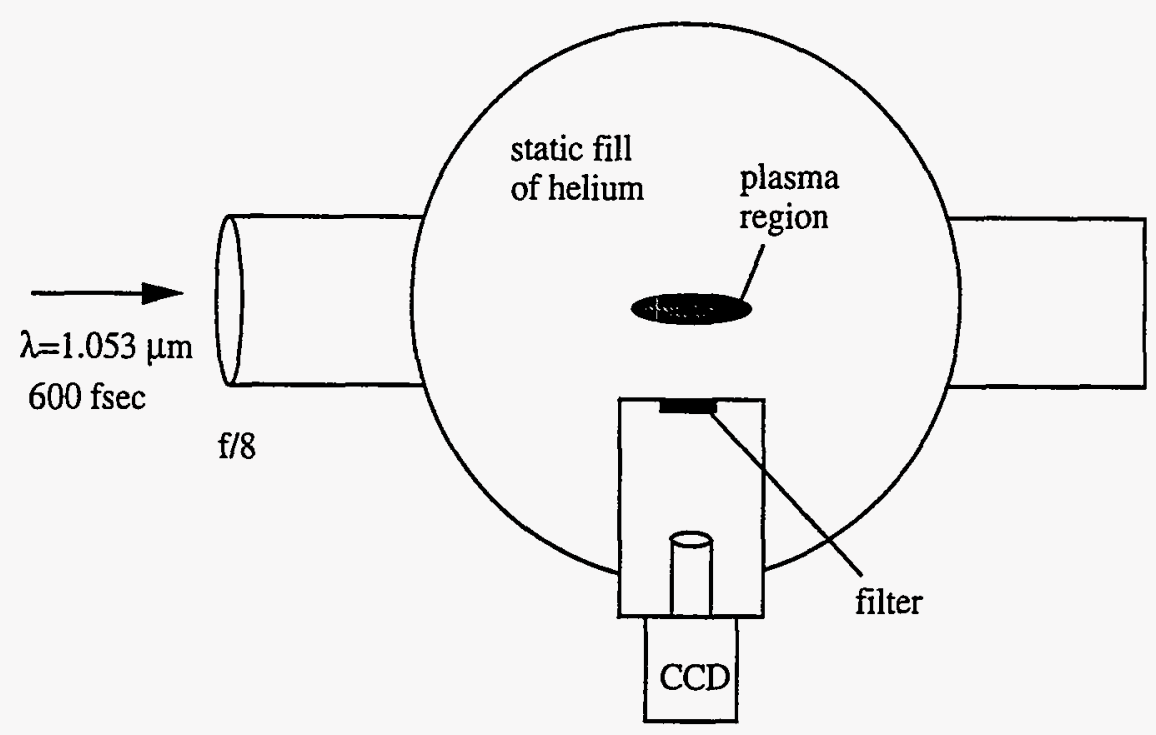

Figure A.5: Experimental configuration used to collect plasma images

interference filters were used to collect different wavelengths of recombination light from the plasma $(600 \mathrm{~nm}, 526 \mathrm{~nm}$ (second harmonic of the laser light, $2 \omega), 500 \mathrm{~nm}$, $400 \mathrm{~nm})$. These filters transmit approximately $10 \mathrm{~nm}$ at the central wavelength. A $1 \omega(1.05 \mu \mathrm{m})$ narrowband interference filter was used to obtain images of the laser as it propagated through the focal region.

A plasma image obtained with a low helium density $\left(2.0\right.$ torr, $\left.1.3 \times 10^{17} \mathrm{~cm}^{-3}\right)$ is shown in figure A.6(a). The laser intensity was $1.7 \times 10^{17} \mathrm{~W} / \mathrm{cm}^{2}$. This image was collected using the $1 \omega$ filter so that only light near the laser wavelength was imaged. The laser propagates from left to right in this image. Figure A.6(b) is a contour plot of the image shown in figure A.6(a). A smooth distribution of light is observed in these intensity contours for both lobes. The intensity of light observed in the image is a measure of the number of scatterers present (i.e., amount of plasma) in the region. The lobe before the focus is slightly brighter and larger than the lobe after the focus. This probably results from refraction effects since pump depletion is not an issue at this density (see figure A.3). Both lobes are brightest along the laser axis, as expected since the laser intensity is highest there. The calculated shape 

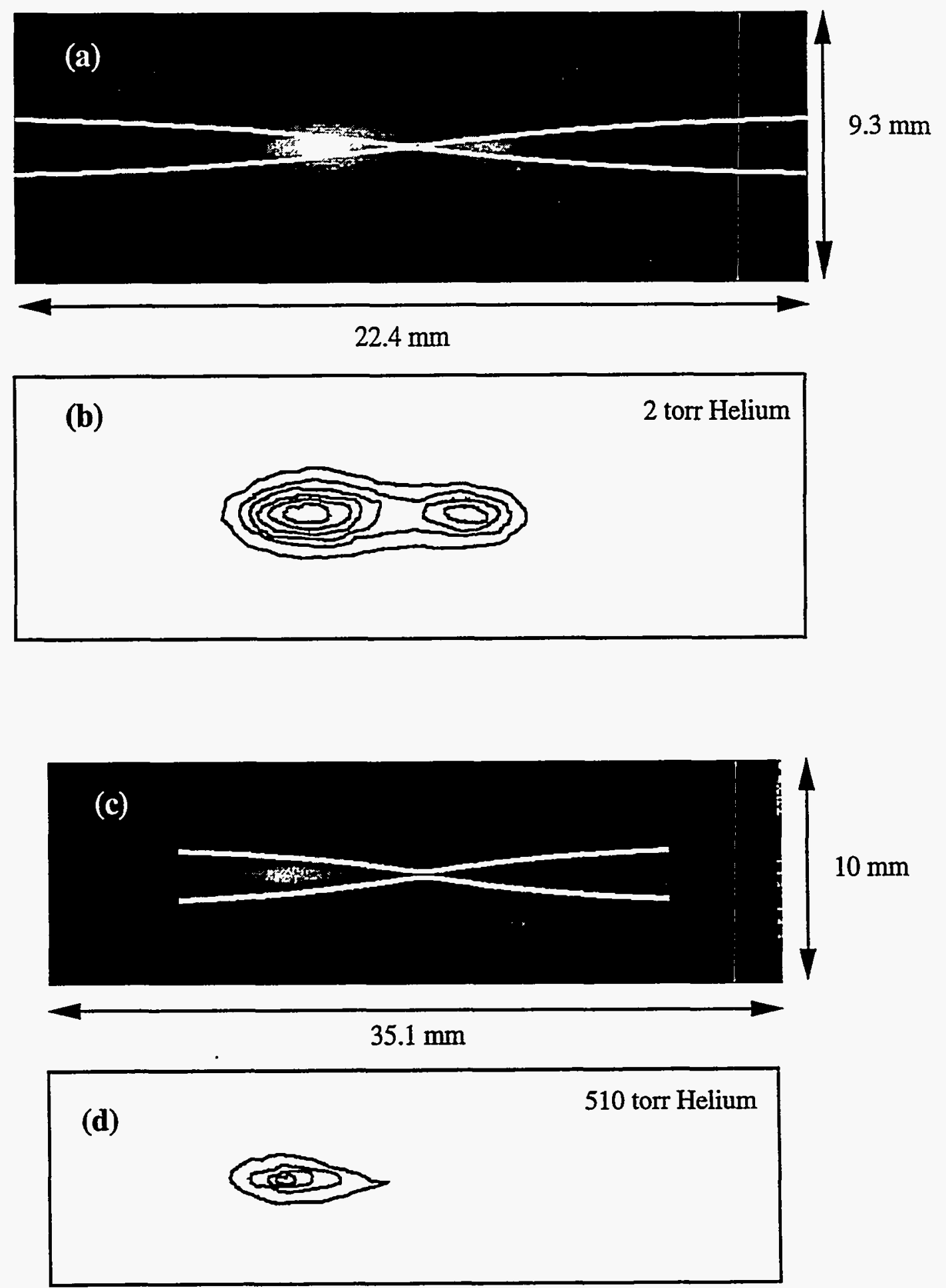

Figure A.6: Plasma images obtained using a $1 \omega$ filter for (a) 2.0 torr helium and (c) 510 torr helium. Contour plots of these images are shown in (b) and (d). 
of the plasma based on equation (A.2), and including the region of $\mathrm{He}^{+}$plasma, is shown in figure A.6(a) (white curve). To calculate this curve, a spot size, $w_{0}$, of 35 $\mu \mathrm{m}$ and a Rayleigh length, $z_{0}$ of $0.5 \mathrm{~mm}$ were used. The spot size used here is larger than typically assumed for this laser, but at the time the images were obtained the focal region was not as well characterized as it was for later experiments.

The predicted shape of the focal region and the observed shape were similar, although the predicted focal region does not extend as far in the radial direction as the observed plasma. This may simply be due to the fact that the experimental laser pulse forms an Airy pattern at the focus and the calculation assumes a Gaussian profile. With an Airy pattern, $84 \%$ of the energy is contained within the central lobe, with the remaining $16 \%$ distributed in the outer concentric rings. For the high laser intensities used here, the energy in these rings can be sufficient to ionize regions of gas to generate plasmas larger than the central lobe alone could produce. It is also possible that the large radial extent of the plasmas results from expansion of the plasma. Since these images are time-integrated, if self-emission (recombination) of the plasma occurs near $1.05 \mu \mathrm{m}$, this light would be observed in the images. There are several wavelengths in the visible and IR region which helium plasmas can emit via recombination. In particular, there are three particularly strong $\mathrm{He} I$ emission lines near $1083 \mathrm{~nm}$. Therefore, the light collected with the $1 \omega$ filter could be collected in the images. For a plasma temperature of $50 \mathrm{eV}$, the helium ions could move approximately $50 \mu \mathrm{m}$ if a recombination time of $10^{-9} \mathrm{sec}$ is assumed. Therefore, some of the radial extent of the observed plasmas could be explained by this recombination light.

Similar images were obtained for higher plasma densities (510 torr, $3.3 \times 10^{19} \mathrm{~cm}^{-3}$ ) when the $1 \omega$ filter was used, as shown in figure A.6(c). The nominal laser intensity for this plasma was $7.2 \times 10^{17} \mathrm{~W} / \mathrm{cm}^{2}$, though refraction reduces this significantly. The white curve in this figure was calculated using equation (A.2). A contour plot of the image is shown in figure A.6(d). Only one lobe is present in this image, the one before "best focus." This is probably due to refraction effects (e.g., ionization induced refraction) and the loss of energy to ionization since the plasma density is 
high. The discussion in section 3.5.1 showed that for the laser system used here, the maximum density for which the laser is not significantly altered is $2 \times 10^{18} \mathrm{~cm}^{-3}$. The density used for the image of figure A.6(c) is 10 times higher than this and the loss of incident energy to ionization is considerable (see figure A.3). Therefore, the lack of plasma after the focus can be accounted for as the reduction in laser intensity caused by ionization and refraction to a level below that necessary for appreciable ionization.

The images obtained experimentally are two-dimensional pictures $(x, z)$ of a three dimensional object $(x, y, z)$. As a result, information about the depth of the plasma has been averaged over. I.e., each pixel on the CCD collects light emitted along $\mathrm{y}$, adding up the emission to produce a net signal at position $(\mathrm{x}, \mathrm{z})$. In order to compare these images with calculation predictions, a recombination "brightness" of the plasma is calculated using equation (A.2) by integrating over the depth of the plasma to obtain the column density of emitters at each $(x, z)$. This produces an image in $x-z$ space. Contour plots of the brightness of these images are shown figure A.7 for the parameters of the images shown in figure A.6. Figure A.7(a) shows the brightness contours obtained assuming a spot size, $\mathrm{w}_{0}$, of $35 \mu \mathrm{m}$ and a peak laser intensity of $5 \times 10^{17} \mathrm{~W} / \mathrm{cm}^{2}$ while figure A.7(c) was obtained with $\mathrm{w}_{0}=35 \mu \mathrm{m}$ and $\mathrm{I}_{0}=1 \times 10^{17} \mathrm{~W} / \mathrm{cm}^{2}$. Comparison with the experimental contour plots shows that the length of the calculated and experimental plasmas agree. The radial extent, however, is much larger in the experiments than it is in the calculation (4-5 times larger). Only one lobe is present in figure A.7(b), while two are predicted by the calculation. As noted earlier, however, in the discussion of figure A.6(c), the absence of the second lobe results from refraction and/or pump depletion (see figure A.3). The calculation does not include either of these effects.

The images of figure A.6 have smooth, Gaussian-like profiles, as shown in figure A.8. The curve in figure A.8(a) is a horizontal lineout of the image shown in figure A.6(a) (i.e., along the direction of propagation) while the curve of figure A.8(b) is a vertical lineout through the left lobe (before the focus). The horizontal lineout shows two smooth Gaussian-like features, one for each lobe of the image. The amplitude of 


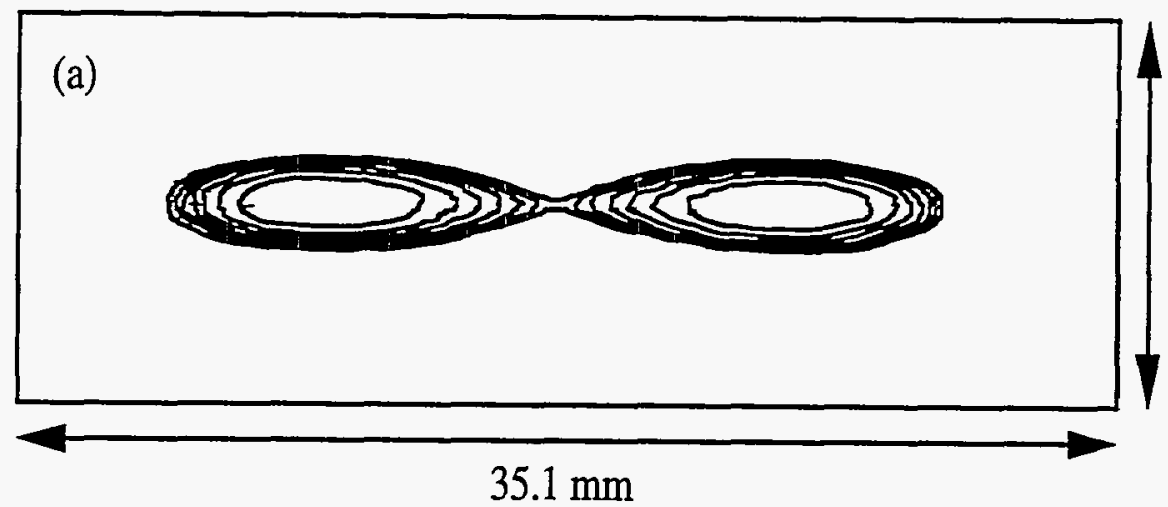

$2.1 \mathrm{~mm}$

(b)
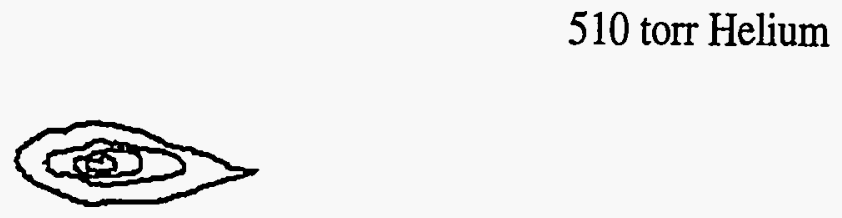

$10 \mathrm{~mm}$

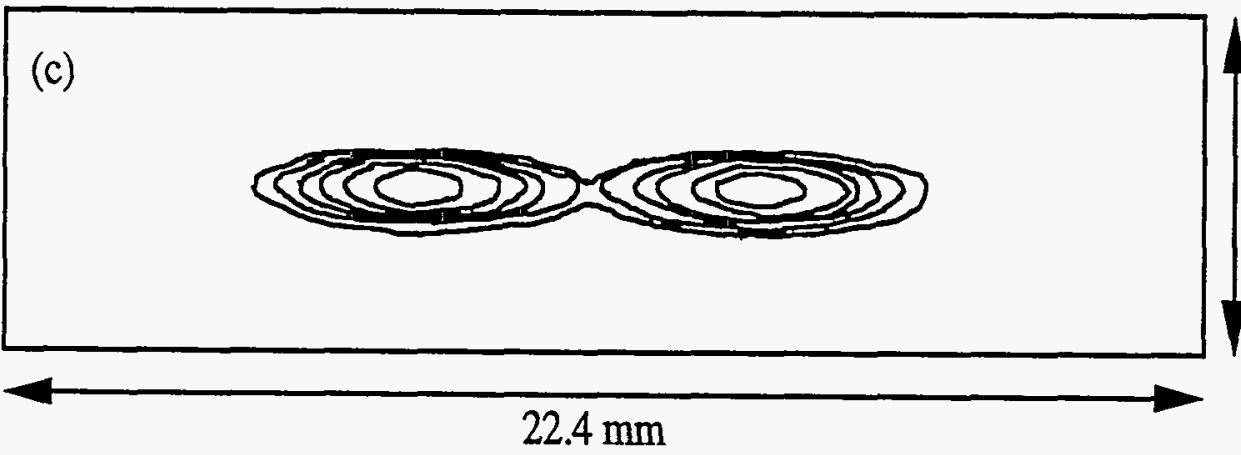

$2.1 \mathrm{~mm}$

(d)
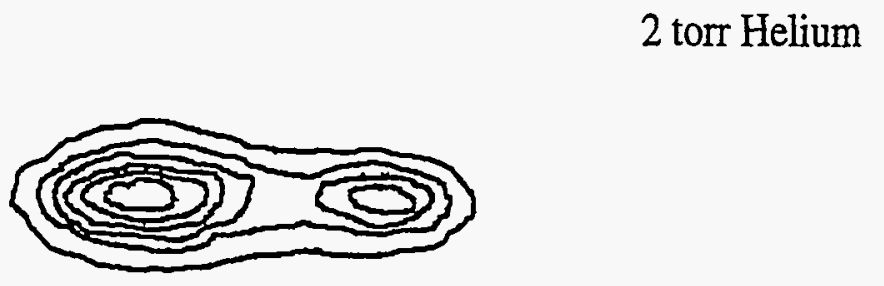

$9.3 \mathrm{~mm}$

Figure A.7: Contour plots of experimental images obtained at 510 torr helium (b) and 2 torr helium (d) and the corresponding calculated recombination brightness contour plots (a) and (c). 

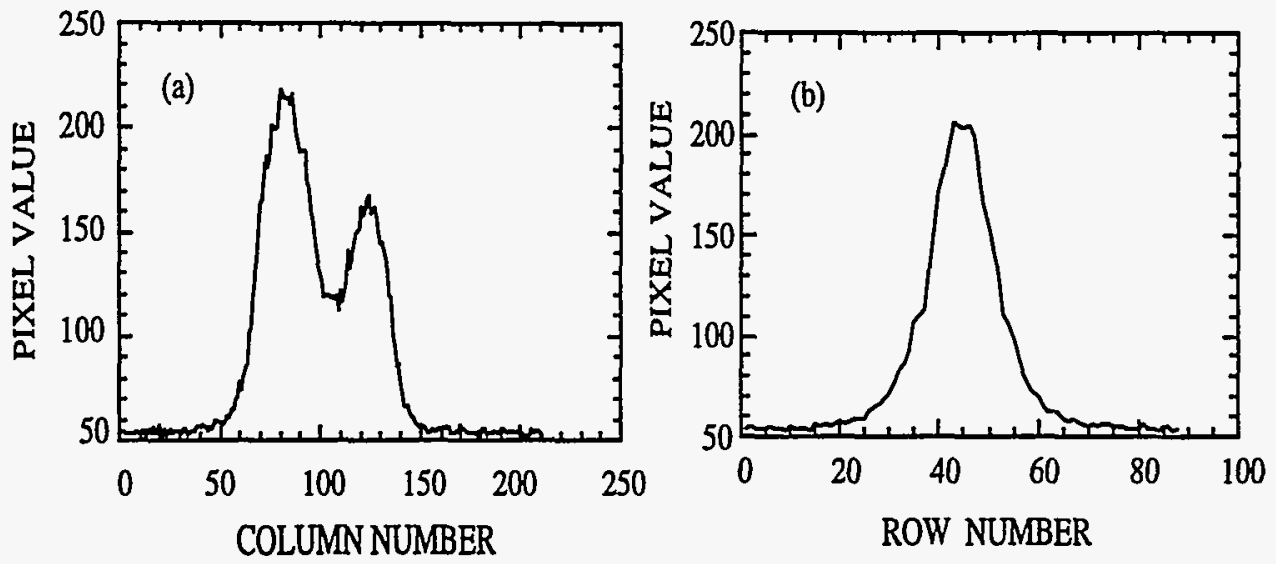

Figure A.8: Horizontal (a) and vertical (b) lineouts of the $1 \omega$ plasma image shown in figure A.6.

the feature representing the lobe before the focus is $33 \%$ larger than the amplitude of the feature after the focus, indicating that the plasma before the focus is thicker. If the light collected in the images is from Thomson scatter, this would imply that the region of brightest emission is the region of highest density (most number of scatterers). From a recombination light standpoint, brighter emission also corresponds to higher density (more electrons and ions recombining). This $33 \%$ difference probably results from the loss of laser energy to refraction since the pump is depleted by less than $1 \%$ for $\mathrm{n}=1.3 \times 10^{17} \mathrm{~cm}^{-3}$ and $\mathrm{I}=1.7 \times 10^{17} \mathrm{~cm}^{-3}$. Refraction of the laser would alter the $\mathrm{f}$-number of the focusing in the plasma. If the f-number of the laser changed from the original $f / 8.2$ to $f / 12$, the column height calculated by equation (A.2) is reduced by $32 \%$. Reduction of the column height reduces the 'depth' of the plasma, and, therefore, the recombination brightness.

Both features in figure A.8(a) are smooth, indicating that the number of scatterers (electron density) varies uniformly throughout the focal region. The decrease in amplitude between the features can be explained by the relatively narrow radial extent of the focus in the focal region. There simply aren't as many electrons to scatter light in this region (or to recombine with the ions). The vertical lineout shown in figure 
A.8(b) is also smooth, indicating that the column density profile varies smoothly in the direction transverse to the laser as well. Lineouts of the plasma image shown in figure A.6(c) also reveal smooth, Gaussian-like behavior.

Horizontal and vertical profiles can be obtained from the calculated recombination "brightness" contours shown in figure A.7. Figure A.9 shows horizontal (a) and vertical (b) lineouts of figure A.7(c). These can be compared with the lineouts of figure A.8. The horizontal lineout of figure A.9(a) illustrates the two-lobe structure of the plasma, with both lobes equal, in contrast to figure A.8(a). A reduction in intensity after the focus (refraction induced change in the f-number), however, would reduce the amplitude of the second feature relative to the first. Neither of these effects was included in the calculation. The vertical lineout of figure A.9 is narrower than that of figure A.8, where the lineout broadens at low amplitudes.
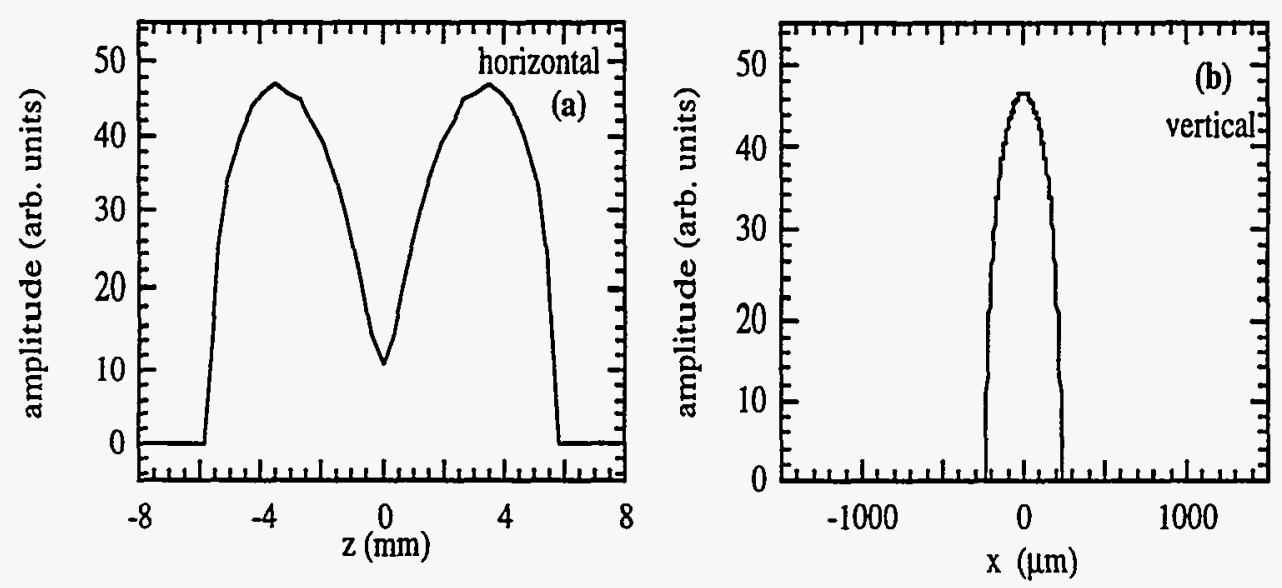

Figure A.9: Horizontal (a) and vertical (b) lineouts of a recombination light calculated image $\left(I_{0}=1 \times 10^{17} \mathrm{~W} / \mathrm{cm}^{2}, \mathrm{w}_{0}=35 \mu \mathrm{m}\right)$.

Plasma images were also obtained by collecting plasma recombination light in different wavelength bands. Images of recombination light are time integrated images and the ions in the plasma may have moved with respect to their positions when the laser was present in the plasma. These images can reveal information about the plasma, however, as did the $1 \omega$ images shown in figure A.6, since recombination can 
only occur where plasma is present. A uniform distribution of plasma should produce a uniform distribution of recombination light. Figure A.10 shows two recombination light images, obtained using a $600 \mathrm{~nm}( \pm 5 \mathrm{~nm})$ filter (a) and a $2 \omega(532 \pm 5 \mathrm{~nm})$ filter (c). These images were obtained with a static fill helium pressure of 400 torr $\left(2.6 \times 10^{19} \mathrm{~cm}^{-3}\right)$. The laser intensities were $6 \times 10^{17} \mathrm{~W} / \mathrm{cm}^{2}$ and $7 \times 10^{17} \mathrm{~W} / \mathrm{cm}^{2}$ for figures A.10(a) and A.10(c) respectively. As was the case for the images of figure A.6, the laser propagates from left to right in these iamges. Contour plots of these images are shown in figures A.10(b) and A.10(d). As observed in the $1 \omega$ image of figure A.6(c), only one feature is present. This is again due to intensity losses resulting from ionization (see figure A.3) and refraction. Unlike the $1 \omega$ image, however, this lobe is not uniform when the recombination light is collected. In fact, the contour plots show a distinctly nonuniform distribution of intensity for both the $600 \mathrm{~nm}$ image and the $2 \omega$ image. This result implies that the plasma is not uniform since the recombination light is not uniform. Horizontal and vertical lineouts of figure A.10(c) are shown in figure A.11. The horizontal lineout (taken along the laser axis) shows variations in the intensity, further illustrating the nonuniformity of the recombination light. The vertical lineout (transverse to the direction of laser propagation), shown in figure A.11(b), shows a smooth profile, however. These lineouts suggest that the plasma is uniform in the radial direction, but not along the axis of laser propagation.

More than one region of bright emission is observable in these images (and in the horizontal lineout of figure A.11(a)), as observed in the scattered $1 \omega$ images of figure A.6. Unlike the images of figure A.6, however, the recombination light images do not show a smooth concentric distribution of intensity. This suggests that filamentation or self-focusing may be occuring in the plasma. These effects are known to produce varying intensties and densities in laser produced plasmas. ([19],[26],[129]) If ponderomotive self-focusing was occuring, for example, there would be regions in the plasma with different densities. [112] This could produce several areas of bright emission since the plasma would be denser (more emitters) in regions with more ions. Therefore, if ponderomotive self-focusing was responsible for the anomalous structure 

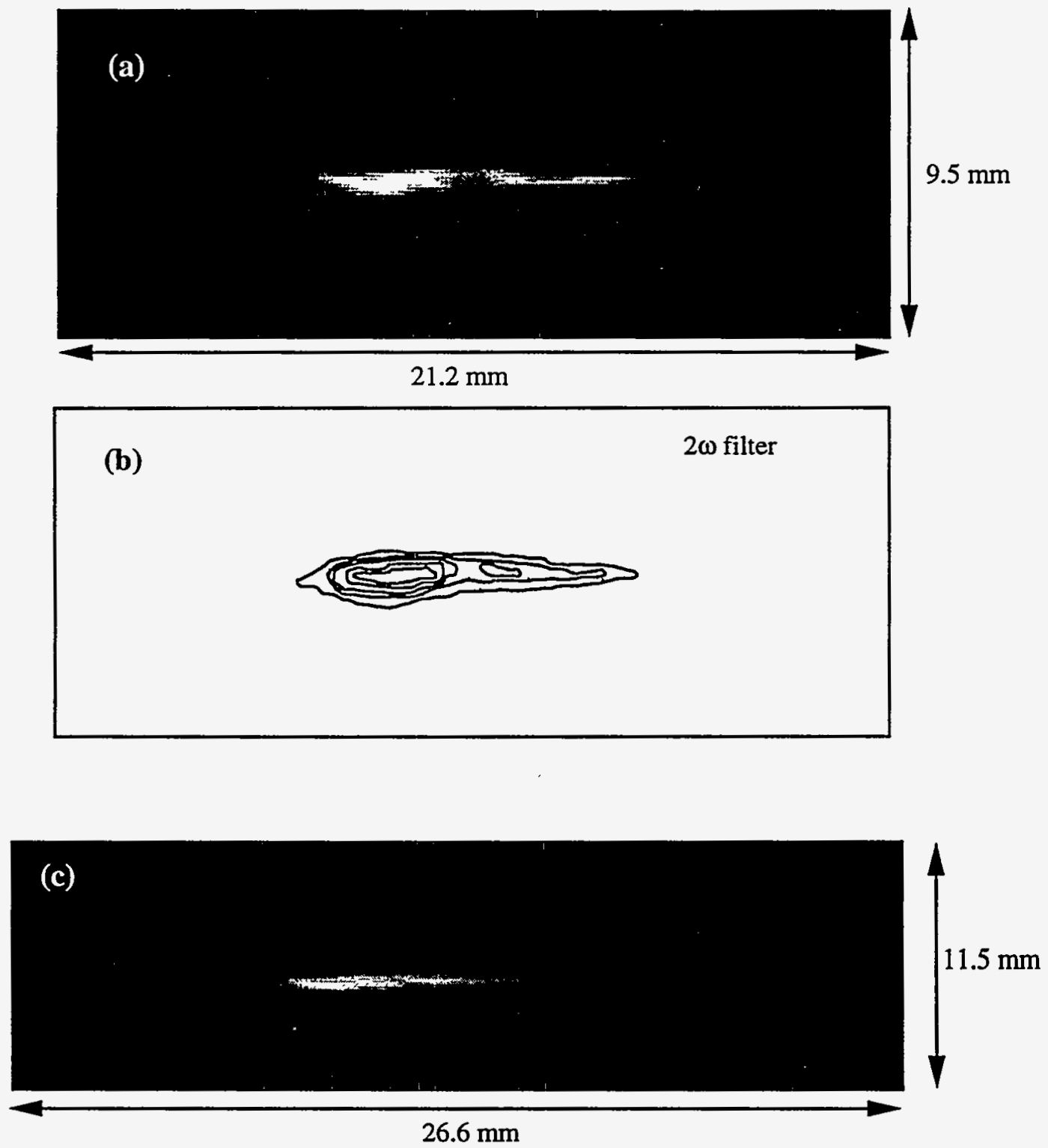

(d) $600 \mathrm{~nm}$ filter

Figure A.10: Plasma images obtained with recombination light of helium plasmas (400 torr). 

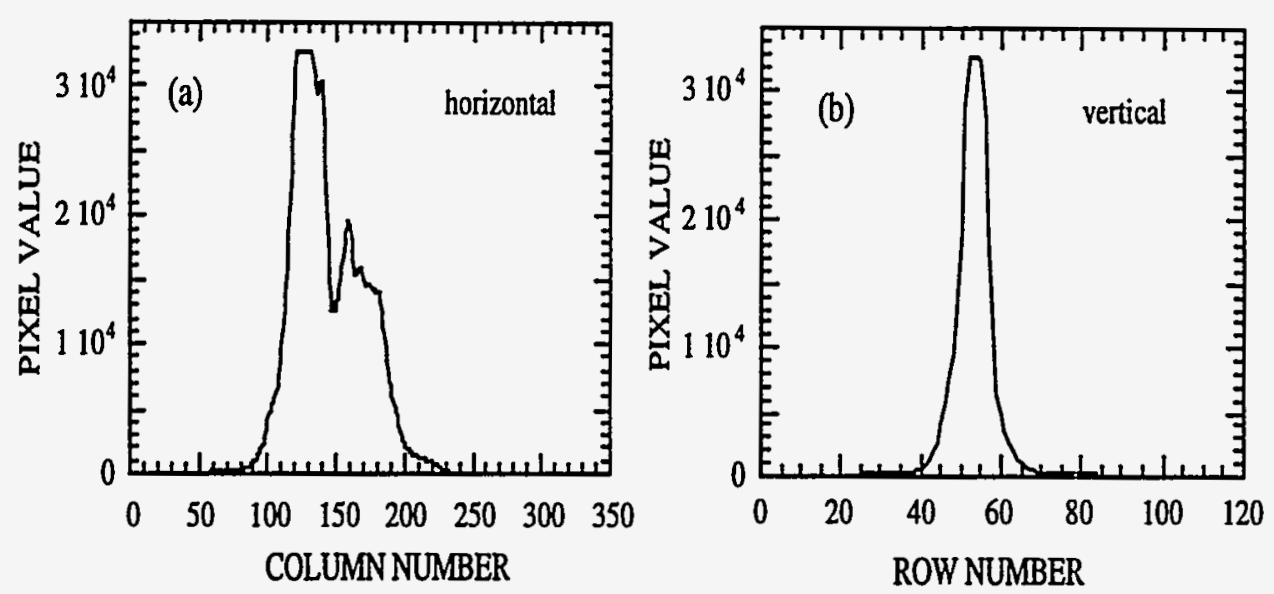

Figure A.11: Horizontal (a) and vertical (b) lineouts of the image shown in figure A.10(c).

in the images obtained with recombination light, one would expect that the brightest emission would not be along the laser axis since this region would be depleted of plasma. One would also expect that the channel generated by this self-focusing would be visible in the $1 \omega$ images since the resolution of the imaging system was approximately $100 \mu \mathrm{m}$. The $1 \omega$ images do not show any channel, however, as shown in figure A.6, and the contour plots and lineouts of the $1 \omega$ images indicate that the only focusing behavior present is from the aspheric $f / 8.2$ lens. Therefore, the structure present in the recombination light images cannot, at this point, be conclusively attibuted to any type of filamentation or self-focusing effect (ponderomotive or otherwise).

To determine if the effects seen in recombination light images were unique to helium, plasma images were also obtained using argon as the target gas. Figure A.12 shows two images obtained using an argon static fill pressure of 200 torr. To assign a single density to these plasmas is not as simple with argon as it is with helium. Recall that for a helium plasma, the saturation intensity for $\mathrm{He}^{2+}$ (fully stripped helium) is only $4 \times 10^{15} \mathrm{~W} / \mathrm{cm}^{2}$. Argon, on the other hand, has 18 charge states, only 8-9 of which are accessible with the intensity available with this laser system. Furthermore, the highest charge states $\left(\mathrm{Ar}^{8+}\right.$ and $\left.\mathrm{Ar}^{9+}\right)$ are only accessible very 
near best focus. This means that as the laser focuses, it encounters many different densities in the plasma. Therefore, assigning a single density value to the plasma is much more difficult with argon than it is with helium. An "average" density can be assigned, however, depending on the incident laser intensity. The average density is determined by assuming an "average charge state." This average charge state is chosen to be the one most representative of the focal region. For the argon static fill images shown here, an average charge state of $\mathrm{Ar}^{7+}$ will be assumed for the high intensity $\left(\geq 2 \times 10^{17} \mathrm{~W} / \mathrm{cm}^{2}\right)$ laser shots. For a static presșure of 200 torr, this gives a plasma density of $4.6 \times 10^{19} \mathrm{~cm}^{-3}$. It should be noted that the incident laser energy lost to ionization of the argon can be appreciable ( $\geq 5 \%$ ) with an incident laser intensity of $\mathrm{I}=5 \times 10^{17} \mathrm{~W} / \mathrm{cm}^{2}$ even for static fill pressures as low as $10^{18} \mathrm{~cm}^{-3}$. This is due to the fact that several electrons are stripped from each neutral argon atom during the ionization. Ionization induced refraction is also an issue here, as it was for high density helium static fills.

The images shown in figure A.12 were obtained with 200 torr of Argon and a $600 \mathrm{~nm}$ filter (a) and a $2 \omega$ filter (c) at laser intensities of $6 \times 10^{17} \mathrm{~W} / \mathrm{cm}^{2}$ and $8 \times 10^{17} \mathrm{~W} / \mathrm{cm}^{2}$, respectively. Contour plots of these images are shown in figures A.12(b) and A.12(d). The laser propagates from left to right in these images. The recombination light from the plasmas show only one lobe of the double lobe predicted behavior and indicate, in both images, that the plasmas are very long $(\approx 2 \mathrm{~cm})$. The images also show structure similar to that seen in the recombination light images of helium plasmas. In fact, in argon, the structure appears more pronounced. The images in figure A.12, and the corresponding contour plots shown in figures A.12(b) and A.12(d), show several localized regions of emission. It should be noted that these images are column averaged; i.e., the depth of the plasma has been averaged in the images. The recombination light from the argon plasmas suggest the possiblity of filamentation generating these localized emission regions, as did the helium recombination light images. The argon images suggest filamentation even more than the 


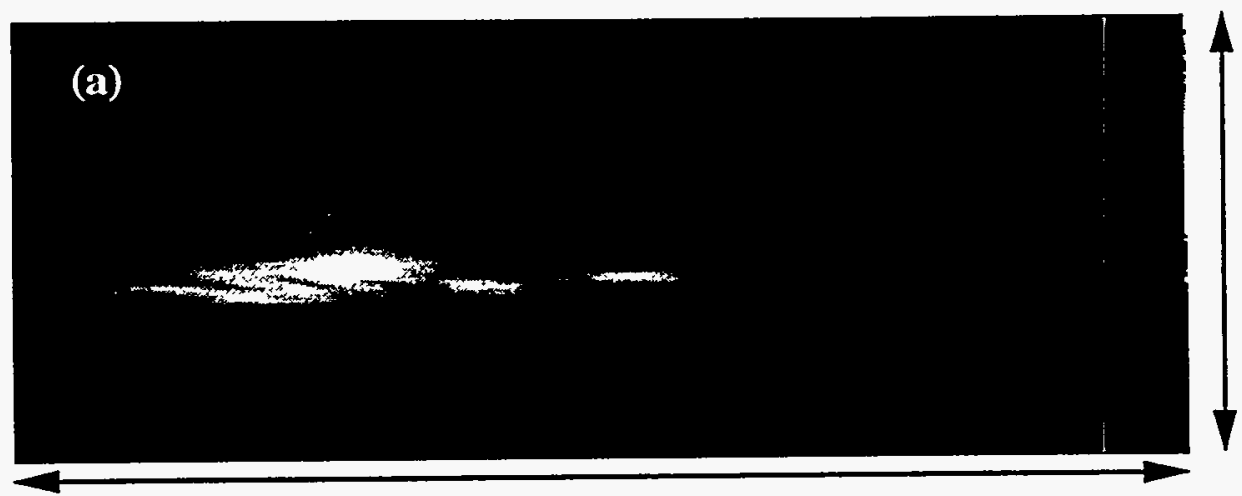

$15.6 \mathrm{~mm}$

$39.4 \mathrm{~mm}$
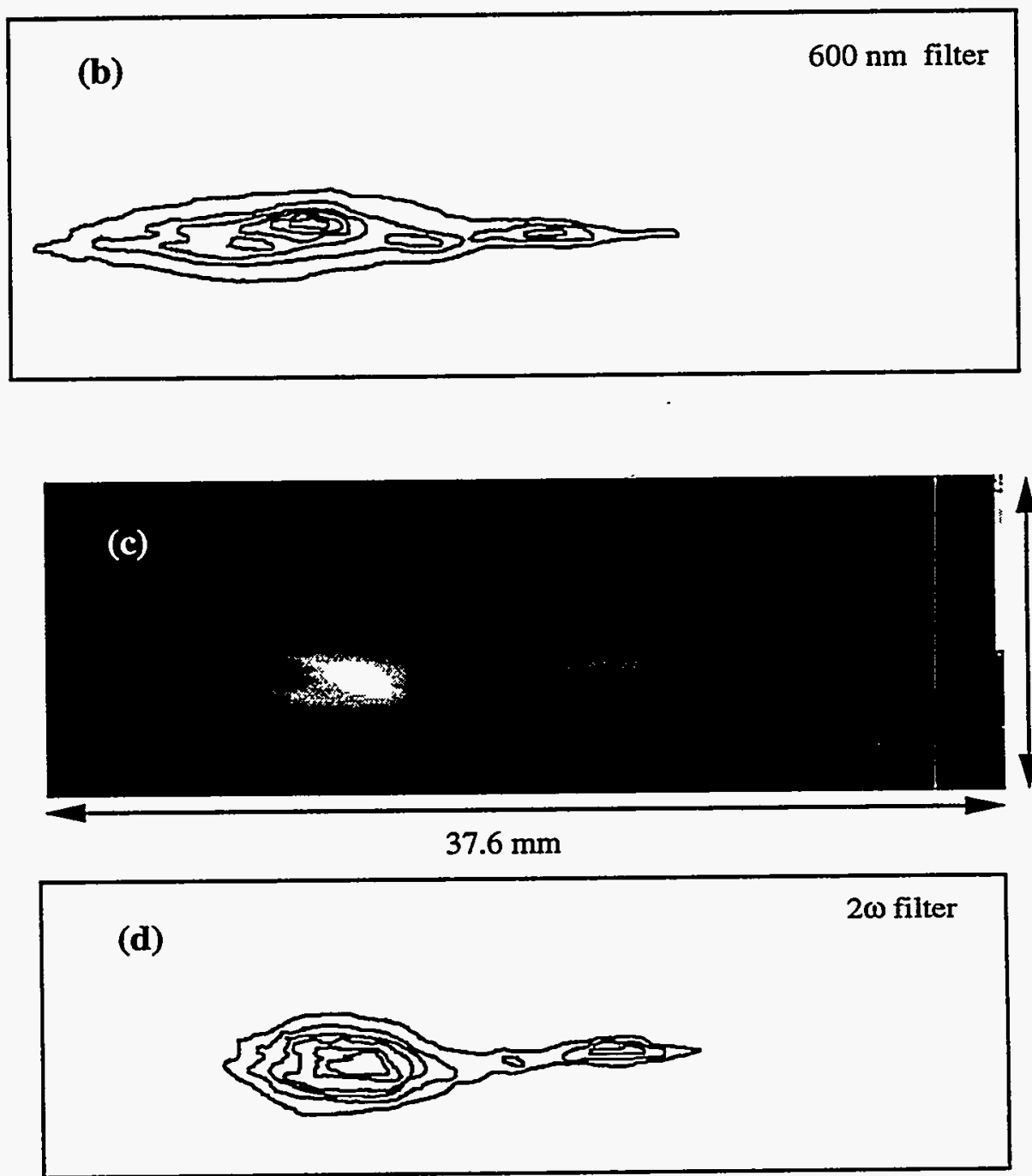

Figure A.12: Recombination light plasma images of Argon plasmas (200 torr). 
helium images did since both images of figure A.12 show relatively narrow extensions of light. Figure A.12(a) appears to have several long, narrow strips of emission. Images of the argon plasmas collected with the $1 \omega$ filter, however, do not show this structure, as was the case with the helium images. The lack of structure in the $1 \omega$ plasma images again reduces the plausibility of the structure resulting from filamentation or self-focusing.
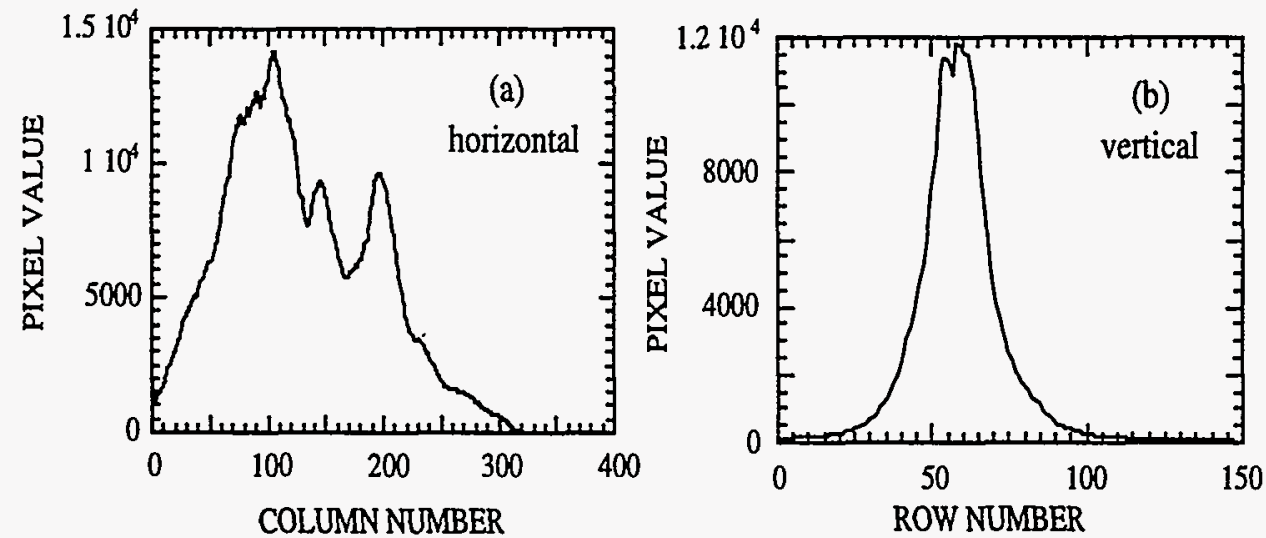

Figure A.13: Horizontal (a) and vertical (b) lineouts of the plasma image shown in figure A.12(a).

Lineouts of the argon plasma image shown in figure A.12(a), the image produced by the $600 \mathrm{~nm}$ recombination light, are shown in figure A.13. The horizontal lineout (along the direction of laser propagation), shown in figure A.13(a), is not smooth. Three distinct peaks are visible in this lineout, indicating that three distinct regions of intense emission were present along the direction of laser propagation. This suggests that the plasma density was not uniform in $\mathrm{z}$ since the brightest recombination emission should arise from the regions of densest plasma, a fact supported by the image from which this lineout was taken. The vertical lineout, taken transverse to the propagation direction, was fairly uniform, indicative of a relatively uniform radial distribution of plasma. This lineout was taken through the brightest portion of the image, however. The distribution is less uniform if a lineout is taken in one of less 
intense regions of the image. Again, the lineouts, both vertical and horizontal, are similar to those obtained with helium plasmas.

The similarities between the helium recombination light images and the argon recombination light images indicate that the mechanism for the structure is not strictly a helium effect or an argon effect. The structure in the images is more pronounced in argon, but there are many differences in the ionization dynamics of these two plasmas, including atom (ion) mass, uniformity of the plasma, and energy lost to ionization.

\section{A.3 Summary}

The size and shape of laser-produced plasmas can be predicted theoretically for comparison with experimentally observed plasmas. The amount of laser energy lost to ionization can also be estimated. For high density plasmas (i.e., $10^{19} \mathrm{~cm}^{-3}$ ), this pump depletion is appreciable for high laser intensity $\left(I \approx 10^{17} \mathrm{~W} / \mathrm{cm}^{2}\right)$ static fill experiments $(\geq 10 \%)$. For a localized region of plasma (i.e., gas jet), however, the pump depletion due to ionization is not important $(\leq 1 \%)$. Images obtained with $1.05 \mu \mathrm{m}$ light for low density helium plasmas resemble those predicted by a gaussian formalism. At higher densities in infinite gas volumes, the shape of the plasma obtained experimentally (at $1 \omega$ ) differs from the theoretically predicted shape. This difference lies mainly in the disappearance of the "lobe" after the focus, an effect explainable through a reduction of available laser intensity resulting from ionization induced refraction and the loss of laser energy to ionization. Plasma images obtained with recombination light $(2 \omega$ and $600 \mathrm{~nm})$ show structure and localized regions of recombination emission, suggestive of nonuniform plasmas. Similar recombination light images were obtained with both helium and argon plasmas. The structure in these images suggests that filamentation or self-focusing may be occuring in the plasmas, but plasma images obtained at $1.05 \mu \mathrm{m}$ do not support this theory. 


\title{
Appendix B
}

* Reprint of reference [29]

Propagation of Intense Subpicosecond Laser Pulses through Underdense Plasmas

\author{
C. A. Coverdale, C. B. Darrow, C. D. Decker \\ Lawrence Livermore National Lab, P.O. Box 808, Livermore, CA 94550 \\ W. B. Mori, K.-C. Tzeng, K. A. Marsh, C. E. Clayton, and C. Joshi \\ Departments of Physics and Electrical Engineering, \\ University of California, Los Angeles, CA 90024 \\ December 22, 1994
}

The propagation of an intense, subpicosecond laser pulse through a substantial length $\left(\mathrm{L} / \lambda \sim 10^{3}\right)$ of an underdense plasma $\left(\mathrm{n} / \mathrm{n}_{\mathrm{c}} \sim 1 \%\right)$ is studied through experiments and computer simulations. For $\mathrm{I}=8 \times 10^{17} \mathrm{~W} / \mathrm{cm}^{2}$ only $55 \%$ of the incident laser light was transmitted through the plasma within the focal cone angle. The decrease in transmission was accompanied by Raman forward scattering as evidenced by the generation of anti-Stokes sidebands and $2 \mathrm{MeV}$ electrons. Simulations show that the majority of the reduction in transmission could be due to Raman forward and side scattering.

PACS numbers: $52.35 . \mathrm{Mw}, 52.40 . \mathrm{Db}, 52.40 . \mathrm{Nk}, 52.65 . \mathrm{Rr}$ 
During the past few years there has been a great deal of theoretical and computational work on the propagation of short-pulse, high-intensity laser pulses through underdense plasmas.[1]-[4] By this we mean pulse lengths less than the diffraction length, the plasma length, and the ion plasma period; intensities approaching $10^{18} \mathrm{~W} / \mathrm{cm}^{2}$ for $1 \mu \mathrm{m}$ light; and plasma densities on the order $1 \%$ of the critical density. At these high intensities and low densities, electron-ion collisions are less important in determining the fraction of the energy that can be absorbed by a plasma of a given length. These laser pulses, however, are susceptible to many collective instabilities which can cause beam breakup. One of these instabilities is stimulated Raman forward scattering (SRS-F) [5], which is the decay of an electromagnetic wave $\left(\omega_{0}, \mathrm{k}_{0}\right)$ into a plasma wave $\left(\omega_{\mathrm{p}}, k_{\mathrm{p}}\right)$ and two forward propagating electromagnetic waves at frequency $\omega_{0}-\omega_{\mathrm{p}}$ (Stokes) and $\omega_{0}+\omega_{\mathrm{p}}$ (anti-Stokes) where $\omega_{\mathrm{p}}{ }^{2}=4 \pi \mathrm{e}^{2} \mathrm{n}_{0} / \mathrm{m}_{\mathrm{e}}$. In this letter, we show through experiments and supporting particle-in-cell (PIC) simulations clear evidence for the onset of SRS-F when a short pulse (600 fsec) high intensity $\left(8 \times 10^{17} \mathrm{~W} / \mathrm{cm}^{2}\right) 1 \mu \mathrm{m}$ laser pulse interacts with an underdense $\mathrm{n}=2 \times 10^{19} \mathrm{~cm}^{-3}$, gas jet plasma approximately one millimeter long. An experimental correlation is found between the onset of SRS-F and a decline in the fraction of the laser energy transmitted in the original focal cone. PIC simulations that closely model the experiment in two-dimensions indicate that most of this reduction in transmission can be accounted for by absorption and scattering losses from SRS at near forward antles. These results may be important for the recently proposed fast-ignitor fusion concept [6], the self-modulated laser wake-field accelerator [3], and optical-field ionized x-ray laser schemes [?].

For conditions found in long pulse experiments, SRS-F had proven to be difficult to observe because the spatial gain across the plasma was limited and the temporal growth time was greater than an ion plasma period. [8] Therefore, there have been few previous experimental observations of the scattered light from SRS-F. [9],[10] In fact, only Turner et. al. [9] detected both the Stokes and anti-Stokes signals (a $0.2 n_{c}$ plasma). Another diagnostic for SRS-F is the detection of energetic electrons 
[11] generated by the plasma wave which results from SRS-F. Joshi et. al. directed detected $1.8 \mathrm{MeV}$ electrons from a $0.2 \mathrm{n}_{c}$ plasma which Turner et. al. correlated the occurrence of the anti-Stokes light with $\mathrm{keV} x$-rays. The simultaneous observation of the anti-Stokes sidebands and energetic electrons is the strongest evidence for the excitation of SRS-F.

For short-pulse lasers, SRS-F is an absolute instability [1],[2],[4] in the frame of the laser pulse. The spatial-temporal theory of SRS-F predicts an asymptotic gain given by [4] $(2 \pi \mathrm{G})^{-1 / 2}$, where $\mathrm{G}=\left[8\left(\mathrm{P} / \mathrm{P}_{\mathrm{c}}\right)\left(\mathrm{L} / \mathrm{L}_{\mathrm{R}}\right)\left(\omega_{\mathrm{p}} \tau\right)\left(\omega_{\mathrm{p}} / \omega_{0}\right)\right]^{1 / 2}, \mathrm{P}_{\mathrm{c}}$ is the critical power necessary for relativistic self-focusing [12],[13], and $\mathrm{L}_{\mathrm{R}}=\pi \mathrm{w}_{0}{ }^{2} / \lambda$ is the Rayleigh length. For linearly polarized light, $\mathrm{P} / \mathrm{P}_{\mathrm{c}}=\mathrm{a}_{0}{ }^{2}\left(\mathrm{w}_{\mathrm{o}}{ }^{2} / 32\right)\left(\omega_{\mathrm{p}}{ }^{2} / \mathrm{c}^{2}\right)$ where $\mathrm{a}_{0}$ is the normalized vector potential of the laser $\mathrm{a}_{0}=\mathrm{eA}_{0} / \mathrm{mc}^{2}$, and $\mathrm{w}_{0}$ is the spot size. Therefore, for short pulses $\left(\omega_{\mathrm{p}} \tau \approx 150\right)$ and tenuous plasmas $\omega_{\mathrm{p}} / \omega_{0}=\left(\mathrm{n} / \mathrm{n}_{\mathrm{c}}\right)^{1 / 2}=0.1$, the laser power $\mathrm{P}$ needs to be of order $\mathrm{P}_{c}$ to observe SRS-F within a Rayleigh length (i.e., $G>10$ ). We emphasize that SRS-F can occur in the absence of relativistic self-focusing and that the amount of SRS-F will continuously increase as $P / P_{c}$ is increased from less than unity to greater than unity. For our laser parameters, the requirement that $\mathrm{P} / \mathrm{P}_{\mathrm{c}}$ be near unity necessitates a plasma density near $10^{19} \mathrm{~cm}^{-3}$ for $1 \mu \mathrm{m}$ light. If the plasma is formed by field-induced ionization of a static fill gas, ionization induced refraction (IIR) limits the maximum plasma density that can be obtained to about $2 \times 10^{18} \mathrm{~cm}^{-3}$. [14] Therefore, to limit refraction and achieve the required density, the laser is focussed into a supersonic gas jet target. For short pulses, stimulated Raman backscatter grows spatially from the front of the pulse with a gain $\exp \left[\left(\mathrm{a}_{0} / 2 \sqrt{2}\right)\left(\omega_{0} / \omega_{\mathrm{p}}\right)^{1 / 2}\left(\omega_{\mathrm{p}} \tau\right)\right.$. [7] This gain is generally large, and the instability eventually becomes strongly coupled [15] but the refelctivity is typically low because it saturates very rapidly at a local position within the laser pulse, limiting the amount of laser energy which can be coherently backscattered.

In the present experiments, a glass laser producing $1.053 \mu \mathrm{m}, 500 \mathrm{fsec}$ pulses with energies up to $3.0 \mathrm{~J}$ was used. The beam ( $8.4 \mathrm{~cm}$ diam., square profile in the near field) was focused by an aspheric lens (69 cm focal length, $\mathrm{f} / 8.2)$ resulting in an Airy pattern 
at the focus [16] with an estimated maximum intensity of $1 \times 10^{18} \mathrm{~W} / \mathrm{cm}^{2} \pm 15 \%\left(\mathrm{a}_{0} \approx\right.$ $0.9)$. In arriving at this result, we have used a gaussian fit to the principal Airy lobe to deduce the effective gaussian beam waist spot sizes [17], $\mathrm{w}_{\mathrm{x}}=13.6 \mu \mathrm{m}, \mathrm{w}_{\mathrm{y}}=20.4 \mu \mathrm{m}$, of the slightly elliptical focal spot. The intensity follows from $I=2 E_{A} /\left(\tau \pi w_{x} w_{y}\right)[17]$ where $\mathrm{E}_{\mathrm{A}}=0.4 \mathrm{E}_{\text {total }}$ is the energy contained in the principal Airy lobe.[16] Placing a gas jet, backed with helium, at the focus results in a tunnel-ionized plasma with a density controlled by varying the backing pressure (200-1000 psi). Using a second identical gas jet, the plasma length was measured to be $0.8 \pm 0.1 \mathrm{~mm}$. [18] The focal depth of the focused beam (distance between half-intensity points on each side of best focus) was measured to be $1.2 \mathrm{~mm}$. While ideal gaussian beam theory is not valid for relating the spot size and focal depth of an Airy beam, the previous discussion of SRS gain is applicable if we substitute beam intensities obtained in this manner when calculating $a_{0}$ and ignore the difference between our measured focal depth and the gaussian Rayleigh range (since in these experiments the plasma length, about 0.8 $\mathrm{mm}$, is less than a focal depth). The frequency shift of the anti-Stokes light was used both as a measure of the plasma density and for monitoring the SRS-F.

Near forward scattered light $\left(5^{\circ}-7^{\circ}\right.$ from the laser axis) was collected and spectrally analyzed using a $0.25 \mathrm{~m}, 150$ grooves/mm grating spectrograph coupled to a liquid nitrogen cooled CCD camera. Because the CCD sensitivity drops off rapidly beyond $1100 \mathrm{~nm}$, the Stokes cascade at $\omega_{0}-\mathrm{n} \omega_{\mathrm{p}}$ could not be measured. This also meant that we could not measure sidescatter red shifted by $\omega_{\mathrm{p}}$ from the pump frequency though it is important in the overall energy balance, as discussed laser. Within a narrow range of laser and gas jet operating parameters, the first and second antiStokes features were clearly observable. Fig. 1(a) shows one such spectrum and the $\Delta \omega$ between the first and second anti-Stokes and between the pump and the first anti-Stokes is the same to within $3 \%$.

Although not shown here, the density inferred from the frequency shift of the first anti-Stokes feature as a function of the backing pressure of the gas jet monotonically 
increased with pressure, as expected. [19] Below 200 psi, the anti-Stokes was not observed because the SRS-F spatio-temporal growth is too small at the lower densities. On the high pressure side, an exponential increase in the amount of light near the pump frequency was observed and the anti-Stokes signal fell below the noise level. This could be due to a combination of diffraction caused by self-focusing from relativistic and plasma wave effects [20] and refraction caused by IIR. [21] Either effect could limit the net interaction length. The increase in scattered pump light occured above $800 \mathrm{psi}$, whereas the anti-Stokes signal was maximum at this pressure ( 5 times larger than signals obtained at $600 \mathrm{psi}$ and $1000 \mathrm{psi}$ ). The anti-Stokes signals were seen to disappear for both $\mathrm{P}<1.2 \mathrm{P}_{\mathrm{c}}$, presumably because there is not a sufficient number of e-foldings of growth, and $\mathrm{P}>5.3 \mathrm{P}_{\mathrm{c}}$, where the laser pulse is intense enough for appreciable self-focusing and electron cavitation to occur. [22] At $800 \mathrm{psi}$, we determined the density of the plasma to be $2 \times 10^{19} \mathrm{~cm}^{-3}$ from the frequency shift of the anti-Stokes signal.

To measure the high energy electrons produced by SRS-F, a $90^{\circ}$ bending magnet was placed eight centimeters past the gas jet in the direct forward path of the laser. Electrons escaping the plasma in an $\mathrm{f} / 4$ cone were deflected by the magnet and detected using a $1 \mathrm{~mm}$ thick silicon surface-barrier detector (SBD). The magnetic field (1.5 kG) was chosen to collect $2.0 \pm 0.1 \mathrm{MeV}$ electrons. The SBD was shielded to minimize false electron signals from ambient $\mathrm{x}$-rays. Nevertheless, there is a slight background x-ray noise level on the SBD. Additional shielding blocked the line of site between the plasma region and teh SBD, ensuring that electrons reaching the SBD were deflected by the magnet. On each laser shot we simultaneously measured the anti-Stokes signals, the $2 \mathrm{MeV}$ electron signal and, in addition, the $\mathrm{x}$-ray signal from a control detector. The control detector, an identical SBD, was placed about $15 \mathrm{~cm}$ from the gas jet $135^{\circ}$ from the laser propagation direction. The electron detector and control detector had identical lead shielding and a $50 \mu \mathrm{m}$ copper foil in front to reduce the $\mathrm{x}$-ray signal. THe $\mathrm{x}$-ray level on the electron SBD was measured by reversing the magnet polarity and correlating the $\mathrm{x}$-ray signals measured on the two detectors. 
The electron-plus-x-ray signal was then measured on the electron detector with the correct polarity $\mathrm{B}$ field while the control detector measured the $\mathrm{x}$-ray contribution. In this manner, the $\mathrm{x}$-ray contribution (typically $10 \%$ ) to the electron signal could be subtracted. The measured correlation of the electron signal and the first anti-Stokes level is shown in Fig. 1(b). These electron measurements were made at or near 800 psi, the pressure at which the anti-Stokes levels were highest. The electron signal was generally seen to increase with an increase in the anti-Stokes level (circles), wheras the $\mathrm{x}$-ray noise level remained more-or-less constant.

In order to estimate the importance of SRS as a collective mechanism that can couple energy into the plasma and also scatter light out of the cone angle of the incident beam, we measured the fraction of the incident laser energy transmitted through the original focal cone as a function of the incident laser energy for a backing pressure of 800 psi (Fig. 1(c)). At low incident laser energy ( $<1 \mathrm{~J}$ ), when no forward propagating collective instabilities were detected, the transmission was very nearly unity. We also measured the total amount of light backscattered in the cone angle of the beam. Even at the highest laser intensities used in this experiment, the total backscattered fraction was less than $2 \%$ and therefore SRS-B and SBS (stimulated Brillouin scattering) [4] are negligible in the overall energy balance. As the incident laser energy was increased beyond $1 \mathrm{~J}\left(\mathrm{P} / \mathrm{p}_{\mathrm{c}} \approx 1.3\right)$, the transmitted fraction began to decrease, as seen by the solid symbols in fig. 1(c). At the highest energies (3.0 $\mathrm{J}, \mathrm{P} / \mathrm{P}_{\mathrm{c}} \approx 5.3$ ), only about $55 \%$ of the incident energy was transmitted in the cone angle of the laser beam. The decrease in teh transmission was accompanied by a rapid increase (4 orders of magnitude) of the energy observed in the first anti-Stokes (open circles). This correlation of the decrease in the transmitted power and the increase in the level of the first anti-Stokes sideband strongly suggests that SRS at near forward angles is playing a role in reducing the transmitted fraction by depositing energy into the plasma and by scattering a substantial amount of laser light out of the cone angle being measured. 
To quantify the role of the Raman forward and sidescatter in reducing the beam transmission, we have carried out two dimensional PIC code simulations of the experiment. With the advent of massively parallel supercomputers it is now possible to model short ulse laser-plasma experiments far more realistically than in the past; i.e., the transverse and longitudinal spatial dimensions, laser pulsewidth, laser to plasma frequency ratio, and propagation distance in the simulation are the same as in the experiments. We have developed a parallelized fully relativistic electromagnetic PIC code which uses the basic algorithm of ISIS on a cyclic mesh.[4],[23] The computational box is a $8192 \times 256$ cartesian grid and the simulations follow $1.2 \times 10^{7}$ particles for $3 \times 10^{4}$ time steps. The main difficulties with quantitatively modeling the experiment are that the noise in the simulation is typically larger than in the experiment and that the simulations are two-dimensional (slab geometry). In the present experiment, however, SRS-B saturates so rapidly that it is rather insensitive to the noise source and the SRS-F noise source is the ponderomotive wake which is independent of the thermal fluctuations. [4]

In order to model the experimental conditions, an estimate of the effective plasma length is required. This estimate was made by performing a series of simulations which covered a range of laser intensities and plasma densities: (A) $\omega_{0} / \omega_{p}=10, a_{0}=0.33$, and $\mathrm{P} / \mathrm{P}_{\mathrm{c}}=0.41 ;(\mathrm{B}) \omega_{0} / \omega_{\mathrm{p}}=10, \mathrm{a}_{0}=0.8$, and $\mathrm{P} / \mathrm{P}_{\mathrm{c}}=2.7$; and $(\mathrm{C}) \omega_{0} / \omega_{\mathrm{p}}=5$, $\mathrm{a}_{0}=0.5$, and $\mathrm{P} / \mathrm{P}_{\mathrm{c}}=4.0$. In each simulation, the laser spot size, $\mathrm{w}_{0}$, was $18 \mu \mathrm{m}$, the laser pulse duration was $600 \mathrm{fsec}$, the plasma was preformed with a uniform density, and the ions were immobile. The evolution of the electron distribution function, the electromagnetic mode spectra, and the laser energy transmitted through the original focal cone were monitored as a function of the distance the head of the laser pulse penetrated into the plasma. The results are summarized in figure $1(d)$ and figure $2(a-d)$.

In Fig. 1(d) we plot the fraction of the total energy remaining in the electromagnetic modes at all frequencies (solid lines) in the forward $2 \pi$ sterradians and the fraction remaining in the original focal cone of th beam (open symbols) as a function 
of the penetration distance for all three simulations. This plot clearly demonstrates that for a fixed penetration distance, both the absorption and scattering losses increase with laser power and plasma density. These results are consistent with the experimental results in that for low SRS-F gain $(G \leq 10)$, very little transmission losses occur while for high SRS-F gain ( $G \geq 10)$, significant transmission losses occur.

To make further quantitative comparisons, we examined the evolution of the electron distribution function and the first two anti-Stokes sidebands. In figure 2 , we show for simulation $B$ the electron distribution function, $f\left(P_{x}\right)$, and the $k$ spectrum of the laser, $\mathrm{E}_{\mathrm{z}}\left(\mathrm{k}_{\mathrm{x}}, \mathrm{k}_{\mathrm{y}}\right)$, after the pulse has penetrated $0.48 \mathrm{~mm}$ and $0.64 \mathrm{~mm}$ into the plasma. Fig. 2(c) and 2(d) show that a substantial amount of light is side-scattered (SRS-S), as illustrated particularly by the STokes line at $k_{x}=9$. This side-scatter is extremely important in the overall energy balance. We calculate the total energy within each anti-Stokes line and find that after $0.64 \mathrm{~mm}(0.48 \mathrm{~mm})$ the ratio of antiStokes/pump=0.06 (0.006) and second anti-Stokes/anti-Stokes $=0.04$ (in the noise). The experimentally measured ratios at $5^{\circ}-7^{\circ}$ for the anti-Stokes/pump and the second anti-Stokes/anti-Stokes were 0.034 and 0.027 respectively. The results from the experiment and the simulations are in reasonable agreement, within the shot-to-shot variation, for a plasma length of $0.64 \mathrm{~mm}$. Importantly, this distance is less than the presumed dimension of the gas jet. In addition, the edge of the electron distribution does not exceed $2 \mathrm{MeV}$ until the laser has penetrated $0.48 \mathrm{~mm}$ into the plasma (fig. 2(a)), and it extends to $5 \mathrm{MeV}$ after $0.64 \mathrm{~mm}$ of plasma, with a few electrons at 20 $\mathrm{MeV}$ (fig. 2(b)). We emphasize that in simulation $\mathrm{A}\left(\mathrm{P} / \mathrm{P}_{\mathrm{c}}=0.41\right)$, no $\mathrm{MeV}$ electrons or higher order anti-Stokes sidebands were observed for laser penetration distances less than $1 \mathrm{~mm}$.

Taking the plasma length to be $0.64 \mathrm{~mm}$, we carried out an additional simulation (D) in which a laser pulse of $\mathrm{a}_{0}=0.8$ was propagated through a $\mathrm{n}=2 \times 10^{19} \mathrm{~cm}^{-3}$ density plasma slab. These are the experimental conditions which gave the maximum transmission losses. In this simulation, only $49 \%$ of the laser light was transmitted through the $\mathrm{f} / 8$ focal cone, indicated in figure $1(\mathrm{~d})$ as a solid triangle. This is in 
reasonable agreement with the experimental measurement of $55 \%$ (solid square in fig. 1(d)). The horizontal error bar on this symbol is indicative of the upper bound of uncertainty in the plasma length. We point out that the $51 \%$ loss in energy can be partitioned as $30 \%$ into Raman plasma waves and $20 \%$ into forward going sidescattered light. The degree to which the simulation and experimental results agree underscores the importance of SRS-F and SRS-S in preventing laser energy from propagating straight through an underdense plasma.

In summary, clear experimental evidence of stimulated Raman forward scatter from a short-pulse high-intensity laser-plasma interaction is presented. The onset of SRS-F is correlated experimentally with the emission of high-energy electrons and a decrease in the transmission of laser energy through the original focal cone. Twodimensional PIC simulations confirm that this decrease in transmission is due mainly to a combination of Raman forward and side scattering.

We would like to thank H. Nguyen, J. Crane, and M.D. Perry for their support of these experiments. This work was performed under the auspices of the U.S. Department of Energy by Lawrence Livermore National Lab under contract no. W-7405ENG-48 and at UCLA under DOE grant number DE-FG03-92ER40727 and LLNL contract numbers B283617 and B283784. 

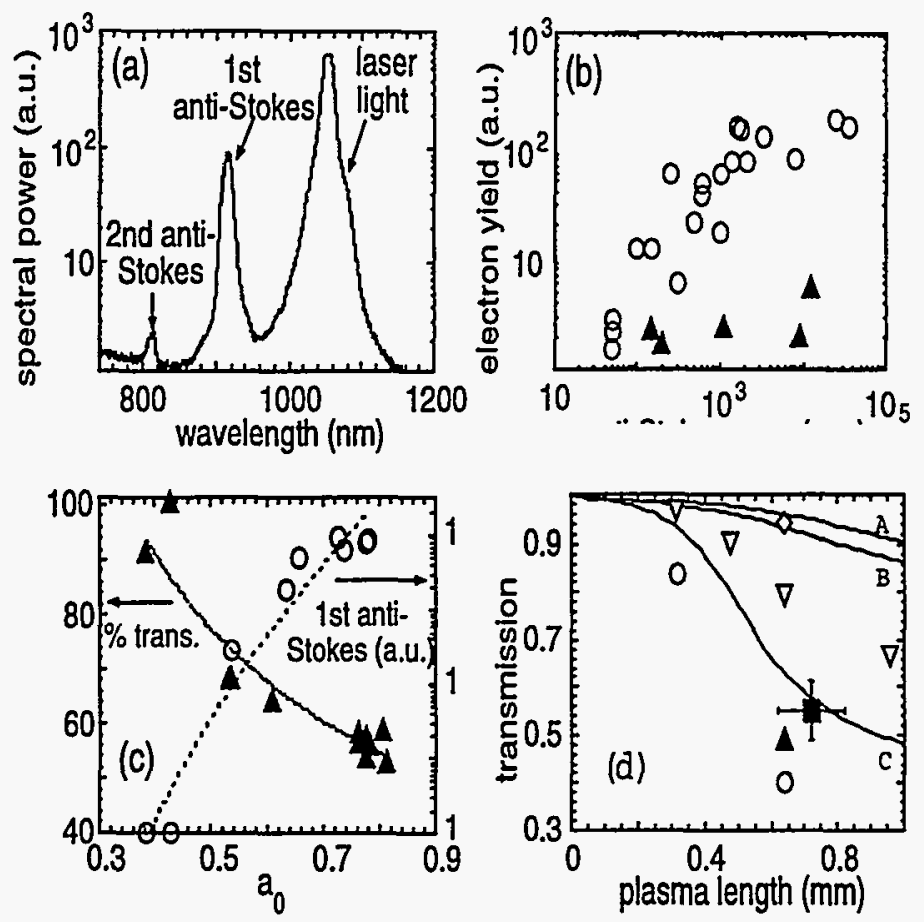

Figure B.1: (a) The frequency spectrum of the forward scattered light $\left(5^{\circ}-7^{\circ}\right.$ from the laser axis) and (b) the correlation of the $2 \mathrm{MeV}$ electron signal with the amplitude of the first anti-Stokes (open circles). The solid triangles represent signal on the electron detector when the magnet polarity was reversed (null tests). (c) The variation of the transmitted laser energy (solid triangles) and the amplitude of the first anti-Stokes (open circles) with $a_{0}$. (d) Transmitted power at all frequencies in the forward $2 \pi$ sterradians (lines) and at all frequencies in the cone angle of the beam (open symbols) versus propagation distance from PIC code simulations. The solid square represents the experimental transmission. The solid triangle is simulation result $\mathrm{D}$. 

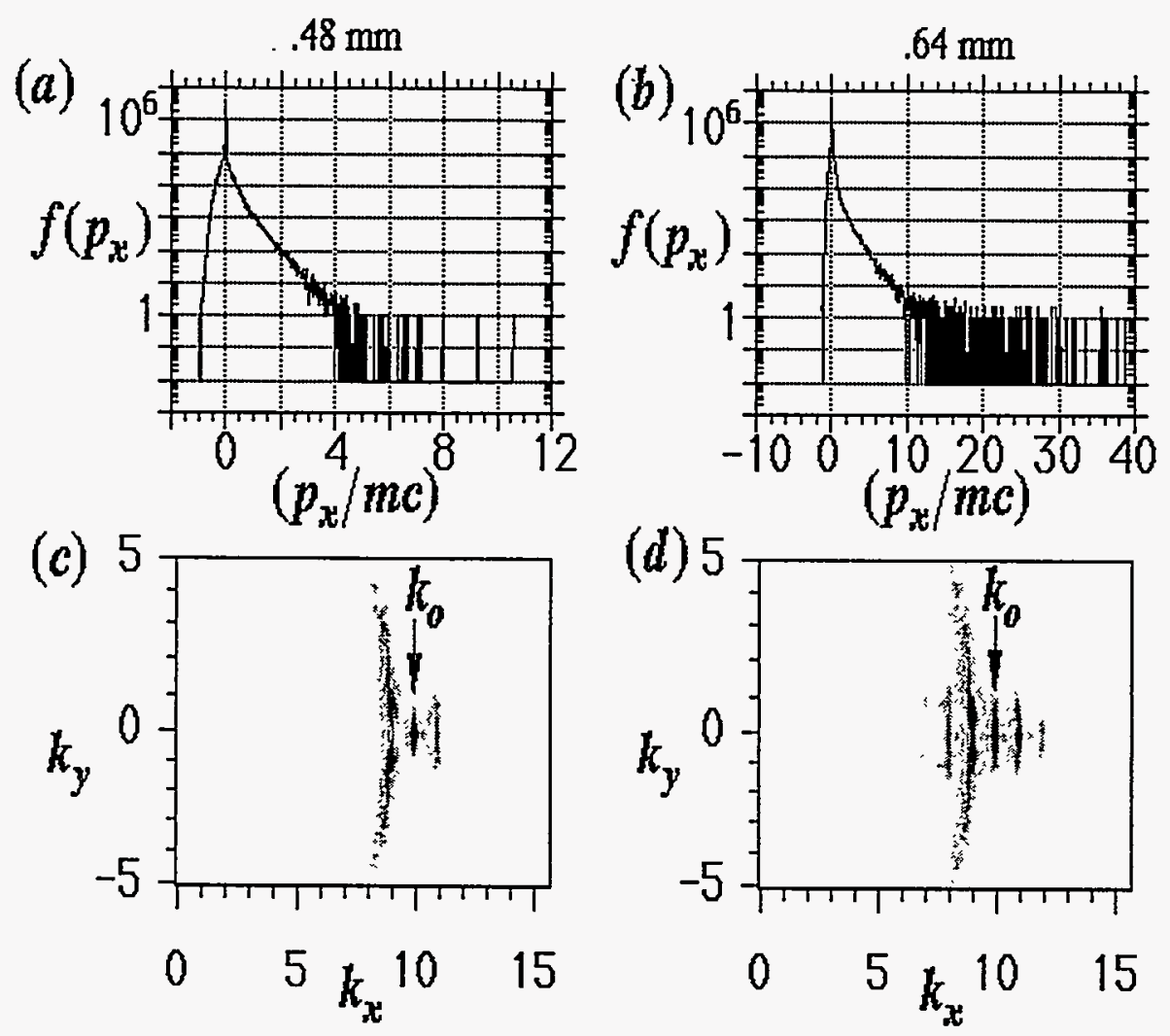

Figure B.2: From simulation (B), the electron distribution function at (a) $0.48 \mathrm{~mm}$ and (b) $0.64 \mathrm{~mm}$ and the corresponding electromagnetic $\mathrm{k}$ spectrum at (c) $0.48 \mathrm{~mm}$ and (d) $0.64 \mathrm{~mm}\left(\mathrm{k}_{\mathrm{x}}\right.$ and $\mathrm{k}_{\mathrm{y}}$ are in units of $\omega_{\mathrm{p}} / \mathrm{c}$. 


\section{Bibliography}

[1] T. Antonsen and P. Mora, Phys. Rev. Lett. 69, 2204 (1992).

[2] P. Sprangle et. al., Phys. Rev. Lett. 69, 2200 (1992); E. Esarey et. al., Phys. Rev. Lett. 72, 2887 (1994).

[3] J. Krall et. al., Phys. Rev. E 48, 2157 (1993); N. E. Andreev et. al., JETP Lett. 55, 577 (1992).

[4] W. B. Mori et. al., Phys. Rev. Lett. 72, 1482 (1994); C. D. Decker et. al., Phys. Rev. E 50, R3338 (1994).

[5] D. W. Forslund et. al., Phys. Fluids 18, 1002 (1975) and references therein; K. Estabrook and W. L. Kruer, Phys. Fluids 26, 1892 (1983).

[6] M. Tabak et. al., Phys. Plasmas 1, 1626 (1994).

[7] P. Amendt et. al., Phys. Rev. Lett. 66, 2589 (1991); N. H. Burnett and P. B. Corkum, J. Opt. Soc. Am. B 6, 1195 (1989).

[8] D. W. Forslund et. al., Phys. Rev. Lett. 54, 558 (1985).

[9] R. E. Turner et. al., Phys. Rev. Lett. 57, 1725 (1986).

[10] S. H. Batha et. al., Phys. Rev. Lett. 66, 2324 (1991); D. M. Villeneuve and H. A. Baldis, Phys. Fluids 31, 1790 (1988); C. Labaune et. al., Phys. Fluids B 2, 166 (1990). 
[11] C. Joshi et. al., Phys. Rev. Lett. 47, 1285 (1981).

[12] C. Max et. al., Phys. Rev. Lett. 33, 209 (1974).

[13] P. Sprangle et. al., IEEE Trans. on Plasma Sci. PS-15, 145 (1983); G. Schmidt and W. Horton, Comments Plasma Phys. Controlled Fusion 9, 85 (1985).

[14] W. P. Leemans et. al., Phys. Rev. A 46, 1091 (1992).

[15] C. B. Darrow et. al., Phys. Rev. Lett. 69, 442 (1992).

[16] M. Born and E. Wolf, Principles of Optics (Pergamon Press, New York, 1964).

[17] A. E. Siegman, Lasers (University Science Books, Mill Valley, CA, 1986).

[18] C. Clayton and M. Everett, accepted Rev. Sci. Instr.

[19] C. A. Coverdale, Ph.D. dissertation, University of California, Davis, 1995 (unpublished).

[20] P. Gibbon and A. T. Bell, Phys. Rev. Lett. 61, 1899 (1988); W. B. Mori et. al., Phys. Rev. Lett. 60, 1298 (1988).

[21] W. M. Wood et. al., Phys. Rev. Lett. 67, 3523 (1991).

[22] G. Sun et. al., Phys. Fluids 30, 526 (1987).

[23] C. D. Decker et. al., Phys. Rev. Lett. 72, 490 (1994). 


\section{Bibliography}

[1] P. Amendt, D. C. Eder, and S. C. Wilks, "X-ray lasing by optical-fieldinduced ionization," Physical Review Letters 66, 2589-2592 (1991).

[2] M. R. Amin, C. E. Capjack, P. Frycz, W. Rozmus, and V. T. Tikhonchuk, "Two-dimensional studies of stimulated Brillouin scattering, filamentation, and self-focusing instabilities of laser light in plasmas," Physics of Fluids B 5, 3748-3764 (1993).

[3] T. M. Antonsen, Jr. and P. Mora, "Self-focusing and Raman scattering of laser pulses in tenuous plasmas", Physical Review Letters 69, 2204-2207 (1992).

[4] T. M. Antonsen, Jr. and P. Mora, "Self-focusing and Raman scattering of laser pulses in tenuous plasmas", Physics of Fluids B 5, 1440-1452 (1993).

[5] S. Augst, D. D. Meyerhofer, D. Strickland, and S. L. Chin, "Laser ionization of noble gases by Coulomb-barrier suppression," Journal of the Optical Society of America B 8, 858-867 (1991).

[6] T. Auguiste, P. Monot, L.-A. Lompré, G. Mainfray, and C. Manus, "Defocusing effects of a picosecond terawatt laser pulse in an underdense plasma," Optics Communications 89, 145-148 (1992).

[7] D. R. Bach, D. E. Casperson, D. W. Forslund, S. J. Gitomer, P. D. Goldstone, A. Hauer, J. F. Kephart, J. M. Kindel, R. Kristal, G. A. Kyrala, 
K. B. Mitchell, D. B. van Hulsteyn, and A. H. Williams, "Intensitydependent absorption in $10.6 \mu \mathrm{m}$ laser illuminated spheres," Physical Review Letters 50, 2082-2085 (1983).

[8] H. A. Baldis, D. M. Villeneuve, B. La Fontaine, G. D. Enright, C. Labaune, S. Baton, Ph. Mounaix, D. Pesme, M. Casanova, and W. Rozmus, "Stimulated Brillouin scattering in picosecond time scales: experiments and modeling," Physics of Fluids B 5, 3319-3327 (1993).

[9] H. C. Barr, T. J. M. Boyd, and G. A. Coutts, "Nonlocal effects and the Raman instabilty," Physical Review Letters 60, 1950-1953 (1988).

[10] C. P. J. Barty, C. L. Gordon III, and B. E. Lemoff, "Multiterawatt 30-fs Ti:sapphire laser system," Optics Letters 19, 1442-1444 (1994).

[11] S. H. Batha, K. S. Bradley, R. P. Drake, K. Estabrook, W. L. Kruer, D. S. Montgomery, and B. A. Remington, "Intensity scaling and saturation of stimulated Raman forward scattering," Physics of Plasmas 1, 1985-1996 (1994).

[12] S. H. Batha, D. S. Montgomery, K. S. Bradley, R. P. Drake, K. Estabrook, and B. A. Remington, "Intensity scaling of stimulated Raman forward scattering in laser-produced plasmas," Physical Review Letters 66, 23242327 (1991).

[13] S. D. Baton, C. Rousseaux, Ph. Mounaix, C. Labaune, B. La Fontaine, D. Pesme, N. Renard, S. Gary, M. Louis-Jacquet, and H. A. Baldis, "Stimulated Brillouin scattering with a 1 ps laser pulse in a preformed underdense plasma," Physical Review E 49, R3602-3605 (1994).

[14] R. L. Berger, E. A. Williams, and A. Simon, "Effect of plasma noise spectrum on stimulated scattering in inhomogeneous plasma", Physics of Fluids B 1, 414-421, (1989). 
[15] D. Bohm and E. P. Gross, "Theory of plasma oscillations. A. Origin of medium-like behavior," Physical Review 75, 1851-1864 (1949).

[16] A. B. Borisov, A. V. Borovskiy, V. V. Korobkin, A. M. Prokhorov, C. K. Rhodes, and O. B. Shiryaev, "Stabalization of relativistic self-focusing of intense subpicosecond ultraviolet pulses in plasmas," Physical Review Letters 65, 1753-1756 (1990).

[17] A. B. Borisov, A. V. Borovskiy, V. V. Korobkin, A. M. Prokhorov, C. K. Rhodes, and O. B. Shiryaev, "Relativistic ponderomotive self-channeling of intense ultrashort laser pulses in a medium," Soviet Physics, JETP 74, 604-615 (1992).

[18] A. B. Borisov, A. V. Borovskiy, V. V. Korobkin, A. M. Prokhorov, O. B. Shiryaev, X. M. Shi, T. S. Luk, A. McPherson, J. C. Solem, K. Boyer, and C. K. Rhodes, "Observation of relativistic and charge-displacement self-channeling of intense subpicosecond ultraviolet $(248 \mathrm{~nm})$ radiation in plasmas," Physical Review Letters 68, 2309-2312 (1992).

[19] A. B. Borisov, A. V. Borovskiy, O. B. Shiryaev, V. V. Korobkin, A. M. Prokhorov, J. C. Solem, T. S. Luk, K. Boyer, and C. K. Rhodes, "Relativistic and charge-displacement self-channeling of intense ultrashort laser pulses in plasmas," Physical Review A 45, 5830-5845 (1992).

[20] M. Born and E. Wolf, Principles of Optics, Pergamon Press, New York, 1964.

[21] F. Brunel, "Not-So-Resonant, Resonant Absorption," Physical Review Letters 59, 52-55 (1987).

[22] S. V. Bulanov, I. N. Inovenkov, V. I. Kirsanov, N. M. Naumova, and A. S. Sakharov, "Nonlinear depletion of ultrashort and relativistically strong laser pulses in an underdense plasma," Physics of Fluids B 4, 1935-1942 (1992). 
[23] S. V. Bulanov, T. J. Esirkepov, and N. M. Naumova, "Properties of spectra of the reflected and transmitted radiation during propagation of relativistically strong laser pulses in underdense plasmas," Proceedings of XII International Conference: Laser Interaction and Related Plasma Phenomena, Osaka, Japan (1995).

[24] N. H. Burnett and G. D. Enright, "Population inversion in the recombination of optically-ionized plasmas," IEEE Journal of Quantum Electronics 26, 1797-1808 (1990).

[25] N. H. Burnett and P. B. Corkum, "Cold plasma production for recombination extreme ultraviolet lasers by optical-field-induced-ionization," Journal of the Optical Society of America B 6, 1195-1199 (1985).

[26] A. J. Campillo, S. L. Shapiro, and B. R. Suydam, "Periodic breakup of optical beams due to self-focusing," Applied Physics Letters 23, 628-630 (1973).

[27] X. L. Chen and R. N. Sudan, "Necessary and sufficient conditions for selffocusing of short ultraintense laser pulse in underdense plasma," Physical Review Letters 70, 2082-2085 (1993).

[28] C. A. Coverdale, C. B. Darrow, R. Jones, W. Sawyer, J. Crane, T. Ditmire, M. D. Perry, and P. C. Filbert, "Production of centimeter-scale, high-density plasmas with a linear gas jet," Review of Scientific Instruments 66 160-163 (1995).

[29] C. A. Coverdale, C. B. Darrow, C. D. Decker, W. B. Mori, K. C. Tzeng, K. A. Marsh, C. E. Clayton, and C. Joshi, "Propagation of intense subpicosecond laser pulses through underdense plasmas," accepted to Physical Review Letters. 
[30] C. A. Coverdale, C. B. Darrow, C. D. Decker, N. Naumova, S. Bulanov, W. B. Mori, and K. C. Tzeng, ".....", submitted to Physical Review Letters.

[31] R. D. Cowan, The Theory of Atomic Structure and Spectra

[32] J. K. Crane, M. D. Perry, D. Strickland, S. M. Herman, and R. W. Falcone, "Coherent and incoherent XUV emission in helium and neon laser-driven plasmas," IEEE Transactions on Plasma Science 21, 82-89 (1993).

[33] J. K. Crane, H. Nguyen, S. C. Wilks, T. Ditmire, C. A. Coverdale, T. E. Glover, M. D. Perry, and Y. Zakharenkov, "Strong anomalous emission from $\mathrm{He}$ and $\mathrm{H}$-like neon in short-pulse laser driven plasmas," submitted to Journal of the Optical Society of America, B.

[34] C. B. Darrow, C. Coverdale, M. D. Perry, W. B. Mori, C. Clayton, K. Marsh, and C. Joshi, "Strongly Coupled Stimulated Raman Backscatter from Subpicosecond Laser-Plasma Interactions", Physical Review Letters 69, 442-445 (1992).

[35] C. B. Darrow, R. P. Drake, D. S. Montgomery, P. E. Young, K. Estabrook, W. L. Kruer and T. W. Johnston, "Experimental studies of stimulated Raman scattering in reactor-size, laser-produced plasmas," Physics of Fluids 3, 1473-1478 (1991).

[36] C. B. Darrow, D. Umstadter, T. Katsouleas, W. B. Mori, C. E. Clayton, and C. Joshi, 'Saturation of beat-excited plasma waves by electrostatic mode coupling," Physical Review Letters 56, 2629-2632 (1986).

[37] C. D. Decker, W. B. Mori, and T. Katsouleas, "Particle-in-cell simulations of Raman forward scattering from short-pulse, high-intensity lasers," Physical Review E , 50, R3338-R3341 (1994). 
[38] C. D. Decker, Ph.D. dissertation, University of California, Los Angeles, 1994.

[39] J. Denavit, "Absorption of high-intensity subpicosecond lasers on solid density targets," Physical Review Letters 69, 3052-3055 (1992).

[40] J. F.Drake, P. K. Kaw, Y. C. Lee, G. Schmidt, C. S. Liu, and M. N. Rosenbluth, "Parametric instabilities of electromagnetic waves in plasmas", The Physics of Fluids 17, 778-785 (1974).

[41] R. P. Drake, P. E. Young, E. A. Williams, K. Estabrook, W. L. Kruer, B. F. Lasinski, C. B. Darrow, H. A. Baldis, and T. W. Johnston, "Laserintensity scaling experiments in long-scalelength, laser-produced plasmas", Physics of Fluids 31, 1795-1802 (1988).

[42] N. A. Ebrahim and C. Joshi, "Electron heating in high intensity $\mathrm{CO}_{2}$ laser-plasma interaction," Physics of Fluids 24, 138-144 (1981).

[43] N. A. Ebrahim, H. A. Baldis, C. Joshi, and R. Benesch, "Hot electron generation by the two-plasmon decay instability in the laser-plasma interaction at $10.6 \mu \mathrm{m}, "$ Physical Review Letters 45, 1179-1182 (1980).

[44] D. C. Eder, P. Amendt, and S. C. Wilks, "Optical-field-ionized plasma x-ray lasers," Physical Review A 45, 6761-6772 (1992).

[45] H. Enge, in Focusing of charged particle optics, A. Septier, edt., Academic Press, New York, p. 203 (1967).

[46] E. Esarey, S. K. Ride, and P. Sprangle, "Nonlinear Thomson scattering of intense laser pulses from beams and plasmas," Physical Review E 48, 3003-3021 (1993).

[47] K. Estabrook, W. L. Kruer, and B. F. Lasinski, "Heating by Raman Backscatter and Forward Scatter", Physical Review Letters 45, 1399-1403 (1980). 
[48] K. Estabrook and W. L. Kruer, "Theory and simulation of onedimensional Raman backward and forward scattering", Physics of Fluids 26, 1892-1903 (1983).

[49] M. J. Everett, A. Lal, D. Gordon, K. Wharton, C. E. Clayton, W. B. Mori, and C. Joshi, "Evolution of stimulated Raman into stimulated Compton scattering of laser light via wave-breaking of plasma waves," Physical Review Letters 74, 1355-1358 (1995).

[50] M. J. Everett, A. Lal, C. E. Clayton, W. B. Mori, T. W. Johnston, and C. Joshi, "Coupling between high-frequency plasma waves in laser-plasma interactions," Physical Review Letters 74, 2236-2239 (1995).

[51] D. W. Forslund, J. M. Kindel, and E. L. Lindman, "Theory of stimulated scattering processes in laser-irradiated plasmas", The Physics of Fluids 18, 1002-1016 (1975).

[52] P. Gibbon and A. R. Bell, "Collisionless absorption in sharp-edged plasmas," Physical Review Letters 68, 1535-1538 (1992).

[53] J. D. Hares and J. D. Kilkenny, "A demonstration of the decrease of fast-electron preheat from laser-produced plasmas with increasing pulse length," Journal of Applied Physics 52, 6420-6422 (1981).

[54] D. E. Hinkel, E. A. Williams, and R. L. Berger. "Temporal evolution of stimulated Brillouin scattering," Physics of Plasmas 1, 2987-3002 (1994).

[55] J. T. Hunt, J. A. Glaze, W. W. Simmons, and P. A. Renard, "Suppression of self-focusing through low-pass spatial filtering and relay imaging," Applied Optics 17, 2053-2057 (1978).

[56] C. Joshi, T. Tajima, J. M. Dawson, H. A. Baldis, and N. A. Ebrahim, "Forward Raman instability and electron acceleration," Physical Review Letters 47, 1285-1288 (1981). 
[57] T. Katsouleas, C. Joshi, J. M. Dawson, F. F. Chen, C. Clayton, W. B. Mori, C. Darrow, and D. Umstadter, "Plasma Accelerators," Laser Acceleration of Particles, AIP Conference Proceedings Number 130, American Institute of Physics, New York, pp. 63-98, 1985.

[58] T. Katsouleas and J. M. Dawson, "Unlimited electron acceleration in laser driven plasma waves," Physical Review Letters 51, 392 -395 (1983).

[59] P. K. Kaw, A. T. Lin, and J. M. Dawson, "Quasiresonant mode coupling of electron plasma waves," Physics of Fluids 16, 1967-1975 (1973).

[60] L. V. Keldysh, "Ionization in the field of a strong electromagnetic wave," Soviet Physics JETP 20, 1307-1314 (1965).

[61] J. C. Kieffer, P. Audebert, M. Chakar, J. P. Matte, H. Pépin, T. W. Johnston, P. Maine, D. Meyerhofer, J. Delettrez, D. Strickland, P. Bado, and G. Mourou, "Short-pulse absorption in very steep plasma density gradients," Physical Review Letters 62, 760-763 (1989).

[62] N. A. Krall and A. W. Trivelpiece, Principles of Plasma Physics, San Francisco Press, San Francisco, CA, 1986.

[63] J. Krall, A. Ting, E. Esarey, and P. Sprangle, "Enhanced acceleration in a self-modulated-laser wake-field accelerator", Physical Review E 48, 2157-2161 (1993).

[64] W. L. Kruer, The Physics of Laser Plasma Interactions, Addison-Wesley Publishing Company, 1988.

[65] W. L. Kruer and K. Estabrook, "JxB heating by very intense laser light," Physics of Fluids 28, 430-432 (1985).

[66] W. L. Kruer, J. M. Dawson, and R. N. Sudan, "Trapped-particle instability," Physical Review Letters 23, 838-841 (1969). 
[67] W. L. Kruer and J. M. Dawson, "Sideband instability," Physics of Fluids 13, 2747-2751 (1970).

[68] W. L. Kruer, "Intense laser plasma interactions: from Janus to Nova," Physics of Fluids B 3, 2356-2366 (1991).

[69] W. L. Kruer, "Efficient energy transfer between fast and slow electron plasma oscillations," Physics of Fluids 15, 2423-2426 (1972).

[70] C. Labaune, H. A. Baldis, E. Fabre, F. Briand, D. M. Villeneuve, and K. Estabrook, "Backward and foward stimulated Raman scattering from thin foil targets with a $0.26 \mu \mathrm{m}$ laser," Physics of Fluids B 2, 166-170 (1990).

[71] W. P. Leemans, C. E. Clayton, K. A. Marsh, and C. Joshi, "Stimulated Compton Scattering from Preformed Underdense Plasmas", Physical Review Letters 67, 1434-1437 (1991).

[72] W. P. Leemans, C. E. Clayton, W. B. Mori, K. A. Marsh, P. K. Kaw, A. Dyson, C. Joshi, and J. M. Wallace, "Experiments and simulations of tunnel-ionized plasmas," Physical Review A 46 1091-1105 (1992).

[73] W. P. Leemans, C. E. Clayton, W. B. Mori, K. A. Marsh, A. Dyson, and C. Joshi, "Plasma physics aspects of tunnel-ionized gases," Physical Review Letters 68, 321-324 (1992).

[74] C. S. Liu and M. N. Rosenbluth, "Parametric decay of electromagnetic waves into two plasmons and its consequences," Physics of Fluids 19, 967-971 (1976).

[75] D.H. Lumb, G. R. Hopkinson, and A. A. Wells, "Performance of CCDs for x-ray imaging and spectroscopy," Nuclear Instruments and Methods in Physics Research 221, 150-158 (1984). 
[76] The coil and windings of this magnet were manufactured by GMW, P.O. Box 2578, Redwood City, CA 94064 (model 3470). The pole pieces were custom built and attached to the magnet.

[77] P. Maine, D. Strickland, P. Bado, M. Pessot, and G. Mourou, "Generation of Ultrahigh Peak Power Pulses by Chirped Pulse Amplification", IEEE Journal of Quantum Electronics 24, 398-403 (1988).

[78] C. E. Max, J. Arons, and A. B. Langdon, "Self-modulation and selffocusing of electromagnetic waves in plasmas," Physical Review Letters 33, 209-212 (1974).

[79] C. E. Max, Physics of the coronal plasma in laser fusion targets; in LaserPlasma Interaction, R. Balian and J. C. Adam, eds., North Holland, Amsterdam, 1982.

[80] C. J. McKinstrie and R. Bingham, "Stimulated Raman forward scattering and the relativistic modulational instability of light waves in rarefied plasma," Physics of Fluids B 4, 2626-2633 (1992).

[81] P. Monot, T. Auguste, L. A. Lompré, G. Mainfray, and C. Manus, "Focusing limits of a terawatt laser in an underdense plasma," Journal of the Optical Society of America B 9, 1579-1584 (1992).

[82] P. Monot, T. Auguste, L. A. Lompré, G. Mainfray, and C. Manus, "Energy measurements of electrons submitted to an ultrastrong laser field," Physical Review Letters 70, 1232-1235 (1993).

[83] W. B. Mori, K.-C. Tzeng, and C. D. Decker, private communication.

[84] W. B. Mori, C. D. Decker, D. E. Hinkel, and T. Katsouleas, "Raman Forward Scattering of Short-Pulse High-Intensity Lasers", Physical Review Letters 72, 1482-1485 (1994). 
[85] W. B. Mori, C. Joshi, J. M. Dawson, D. W. Forslund, and J. M. Kindel, "Evolution of self-focusing of intense electromagnetic waves in plasma," Physical Review Letters 60, 1298-1301 (1988).

[86] S. P. Obenschain, C. J. Pawley, A. N. Mostovych, J. A. Stamper, J. H. Gardner, A. J. Schmitt, and S. E. Bodner, "Reduction of Raman scattering in a plasma to convective levels using induced spatial incoherence," Physical Review Letters 62, 768-771 (1989).

[87] A. A. Offenberger, W. Blyth, A. E. Dangor, A. Djaoui, M. H. Key, Z. Najmudin, and J. S. Wark, "Electron temperature of optically ionized gases produced by high intensity $268 \mathrm{~nm}$ laser radiation," Physical Review Letters 71, 3983-3986 (1993).

[88] E. Ott, W. M. Manheimer, and H. H. Klein, "Stimulated Compton scattering and self-focusing in the outer regions of a laser-fusion plasma," Physics of Fluids 17, 1757-1761 (1974).

[89] F. G. Patterson, M. D. Perry, and J. T. Hunt, "Design and performance of a multiterawatt, subpicosecond neodymium:glass laser", Journal of the Optical Society of America B 8, 2384-2391 (1991).

[90] M. D. Perry, C. B. Darrow, C. A. Coverdale, and J. K. Crane, "Measurement of the local electron density by means of stimulated Raman scattering in a laser-produced gas jet plasma", Optics Letters 17, 523525 (1992).

[91] M. D. Perry, F. G. Patterson, and J. Weston, "Spectral shaping in chirped-pulse amplification", Optics Letters 15, 381-383 (1990).

[92] M. D. Perry, Ph.D. Dissertation, University of California, Berkeley, 1987. 
[93] D. W. Phillion, D. L. Banner, E. M. Campbell, R. E. Turner, and K. G. Estabrook, "Stimulated Raman scattering in large plasmas," Physics of Fluids 25, 1434-1443 (1982).

[94] W. H. Press, B. P. Flannery, S. A. Teukolsky, and W. T. Vetterling, Numerical Recipes: The Art of Scientific Computing, Cambridge University Press, 1986.

[95] S. C. Rae, "Ionization-induced defocusing of intense laser pulses in highpressure gases", Optics Communications 97, 25-28 (1993).

[96] C. J. Randall, J. R. Albritton, and J. J. Thomson, "Theory and simulation of stimulated Brillouin scatter excited by nonabsorbed light in laser fusion systems," Physics of Fluids 24, 1474-1484 (1981).

[97] R. Rankin, C. E. Capjack, N. H. Burnett, and P. B. Corkum, "Refraction effects associated with multiphoton ionization and ultrashort-pulse laser propagation in plasma waveguides," Optics Letters 16, 835-837 (1991).

[98] M. N. Rosenbluth, "Parametric instabilities in inhomogeneous media," Physical Review Letters 29, 565-567 (1972).

[99] A. S. Sakharov and V. I. Kirsanov, "Theory of Raman scattering for a short ultrastrong laser pulse in a rarefied plasma",Physical Review E 49,3274-3282 (1994).

[100] E. S. Sarachik and G. T. Schappert, "Classical theory of the scattering of intense laser radiation by free electrons," Physical Review D 1 2738-2753 (1970).

[101] W. Seka, E. A. Williams, R. S. Craxton, L. M. Goldman, R. W. Short, and K. Tanaka, "Convective stimulated Raman scattering instability in UV laser plasmas," Physics of Fluids 27, 2181-2186 (1984). 
[102] G. Shvets, Massachusetts Institute of Technology, private communication.

[103] A. E. Siegman, Lasers, University Science Books, Mill Valley, CA (1986).

[104] R. E. Slusher and C. M. Surko, "Study of density fluctuations in plasmas by small-angle $\mathrm{CO}_{2}$ laser scattering," Physics of Fluids 23 472-490 (1980).

[105] J. C. Solem, T. S. Luk, K. Boyer, and C. K. Rhodes, "Prospects for $\mathrm{X}$-Ray Amplification with Charge-Displacement Self-Channeling", IEEE Journal of Quantum Electronics 25, 2423-2430 (1989).

[106] P. Sprangle, C. M. Tang, and E. Esarey, "Relativistic self-focusing of short-pulse radiation beams in plasmas," IEEE Transactions on Plasma Science PS-15, 145-153 (1987).

[107] P. Sprangle, E. Esarey, A. Ting, and G. Joyce, "Laser wakefield acceleration and relativistic optical guiding," Applied Physics Letters 53, 21462148 (1988).

[108] P. Sprangle, E. Esarey, J. Krall, and G. Joyce, "Propagation and guiding of intense laser pulses in plasmas," Physical Review Letters 69, 2200-2203 (1992).

[109] K. H. Spatschek, "Self-focusing of electromagnetic waves as a result of relativistic electron-mass variation," Plasma Physics 18, 293-303 (1977).

[110] D. Strickland and G. Mourou, "Compression of amplified chirped optical pulses", Optics Communications 56, 219-221 (1985).

[111] B. C. Stuart, S. Herman, and M. D. Perry, "Chirped-pulse amplification in Ti:sapphire beyond $1 \mu \mathrm{m}$," IEEE Journal of Quantum Electronics 31, 528-538 (1995). 
[112] A. Sullivan, H. Hamster, S.P. Gordon, R. W. Falcone and H. Nathel, "Propagation of intense, ultrashort laser pulses in plasmas," Optics Letters 19, 1544-1546 (1994).

[113] A. Sullivan, H. Hamster, H. C. Kapteyn, S. Gordon, W. White, H. Nathel, R. J. Blair, and R. W. Falcone, "Multiterawatt, 100 fs laser," Optics Letters 16, 1406-1408 (1991).

[114] G. Z. Sun, E. Ott, Y. C. Lee, and P. Guzdar, "Self-focusing of short intense pulses in plasmas," Physics of Fluids 30, 526-532 (1987).

[115] M. Tabak, J. Hammer, M. E. Glinsky, W. L. Kruer, S. C. Wilks, J. Woodworth, E. M. Campbell, and M. D. Perry, "Ignition and high gain with ultrapowerful lasers," Physics of Plasmas 1, 1626-1634 (1994).

[116] T. Tajima and J. M. Dawson, "Laser Electron Accelerator", Physical Review Letters 43, 267-270 (1979).

[117] J. J. Thomson, "Stimulated Raman scatter in laser fusion target chambers," Physics of Fluids 21, 2082-2085 (1978).

[118] R. E. Turner, K. Estabrook, R. P. Drake, E. A. Williams, H. N. Kornblun, W. L. Kruer, and E. M. Campbell, "Observation of Forward Raman Scattering in Laser-Produced Plasmas", Physical Review Letters 57, 1725-1728 (1986).

[119] K. C. Tzeng, C. D. Decker, W. B. Mori, and T. Katsouleas, "The nonlinear behavior of self-modulation, self-focusing, and filamentation of shortpulse, high-intensity lasers," Bulletin of the American Physical Society 39, 1583 (1994).

[120] D. M. Villeneuve and H. A. Baldis, "Observation of forward Raman scattering enhanced by backward Raman scattering in a laser plasma", Physics of Fluids 31, 1790-1794 (1988). 
[121] D. M. Villeneuve, R. L. Keck, B. B. Afeyan, W. Seka, and E. A. Williams, "Production of hot electrons by two-plasmon decay instability in uv laser plasmas," Physics of Fluids 27, 721-725 (1984).

[122] C. J. Walsh, D. M. Villeneuve, and H. A. Baldis, "Electron PlasmaWave Production by Stimulated Raman Scattering: Competition with Stimulated Brillouin Scattering," Physical Review Letters 53, 1445-1448 (1984).

[123] R. G. Watt, R. D. Brooks, and Z. A. Pietrzyk, "Observation of stimulated Raman backscatter from a preformed, underdense plasma," Physical Review Letters 41, 170-173 (1978).

[124] S. C. Wilks, W. L. Kruer, M. Tabak, and A. B. Langdon, "Absorption of ultra-intense laser pulses," Physical Review Letters 69, 1383-1386 (1992).

[125] S. C. Wilks, W. L. Kruer, E. A. Williams, P. Amendt, and D. C. Eder, "Stimulated Raman backscatter in ultraintense, short pulse laser-plasma interactions," Physics of Plasmas 2, 274-279 (1995).

[126] S. C. Wilks, in preparation.

[127] W. M. Wood, C. W. Siders, and M. C. Downer, "Measurement of femtosecond ionization dynamics of atmospheric density gases by spectral blueshifting," Physical Review Letters 67, 3523-3526 (1991).

[128] F. C. Young, M. J. Herbst, C. K. Manka, S. P. Obenschain, and J. H. Garder, "Increased hot-electron production at quarter-critical density in long-scale-length laser-plasma interactions," Physical Review Letters 54, 2509-2512 (1985).

[129] P.E. Young, "Characterization of filamentation in laser-produced plasmas," Comments on Plasma Physics and Controlled Fusion 12, 53-67 (1988). 
[130] P. E. Young, H. A. Baldis, and K. G. Estabrook, "Scattered light near the laser wavelength from Nova 2-color experiments," Physics of Fluids B 3, 1245-1249 (1991).

[131] J. Zhou, C. P. Huang, M. M. Murnane, and H. C. Kapteyn, "Amplification of 26-fs, 2-TW pulses near the gain-narrowing limit in Ti:sapphire," Optics Letters 20, 64-66 (1995). 